\title{
Total Synthesis of Caesalpinnone A and Caesalpinflavan B: Evolution of a Concise Strategy
}

\author{
Jacob C. Timmerman, Noah J. Sims and John L. Wood* \\ Department of Chemistry and Biochemistry, Baylor University, One Bear Place 97248, \\ Waco, Texas, 76798, United States. \\ Email: John L Wood@Baylor.edu
}

\section{Table of Contents}

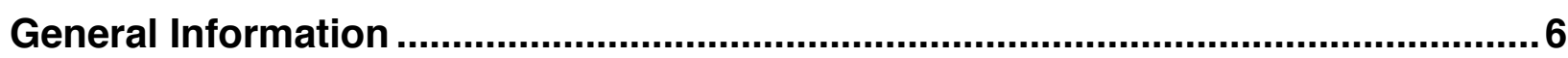

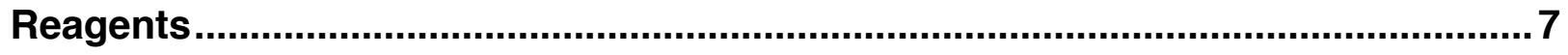

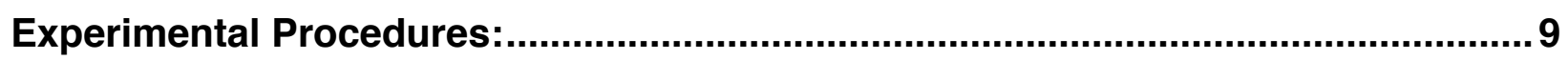

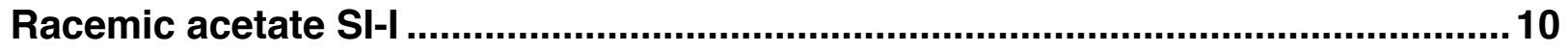

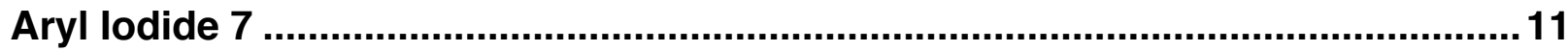

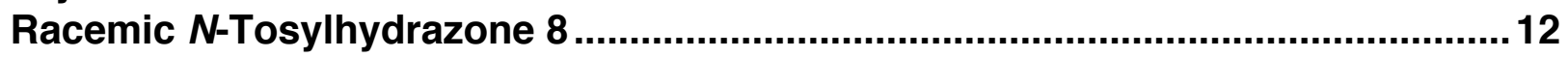

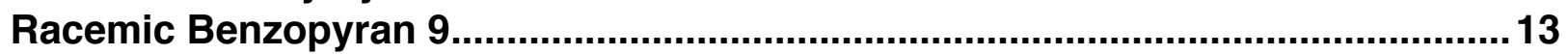

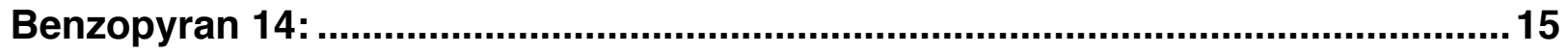

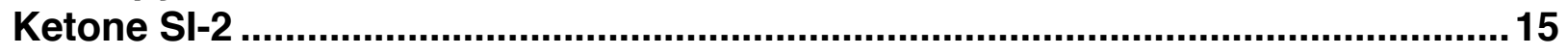

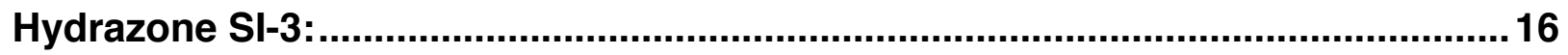

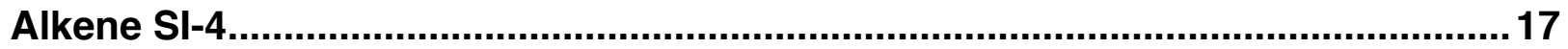

Table S1. Optimization Trials for the coupling of SI-3 and 7 to SI-4. ..................18

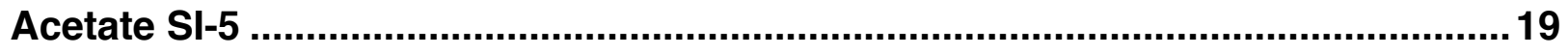

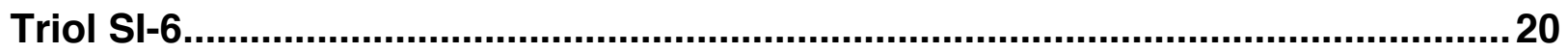

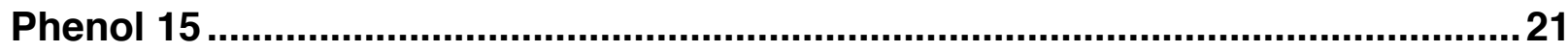

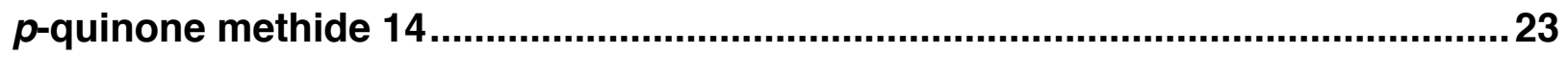

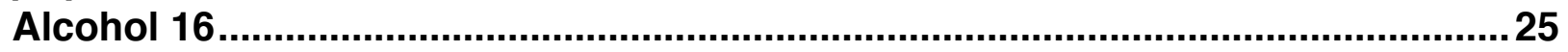

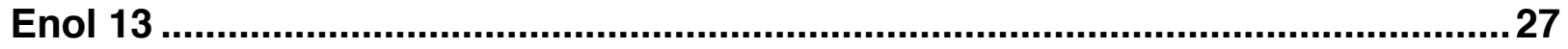

Alcohol 12

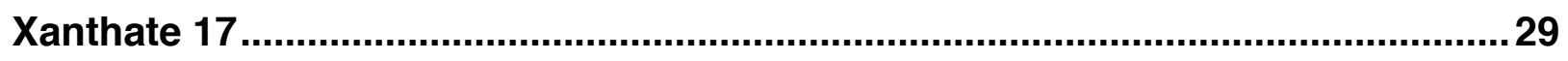

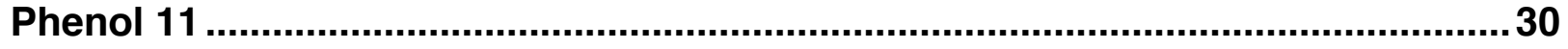

Dihydropyrans 10a and 10b: 


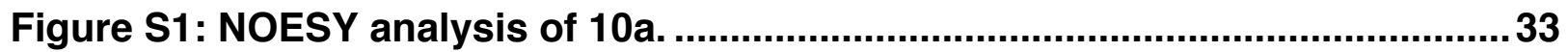

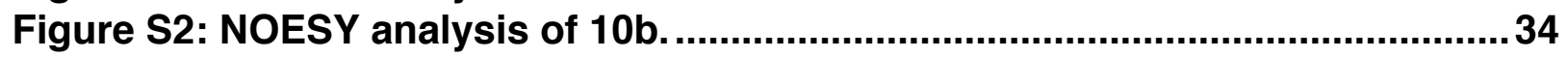

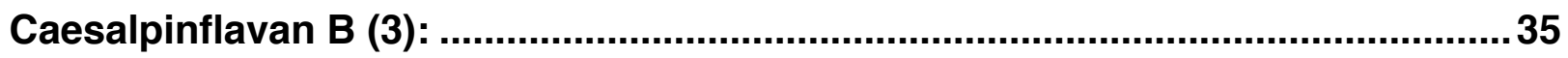

Table S2. Comparison of ${ }^{1} \mathrm{H}$ chemical shifts and multiplicities between natural 3 .

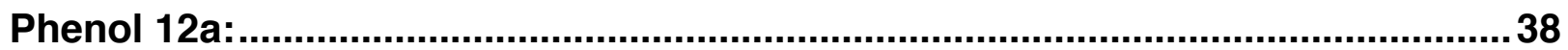

p-quinol 20a:

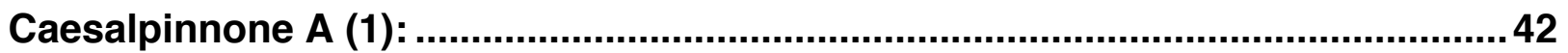

Table S3. Comparison of ${ }^{1} \mathrm{H}$ chemical shifts and multiplicities between natural 1.

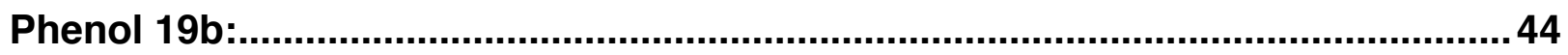

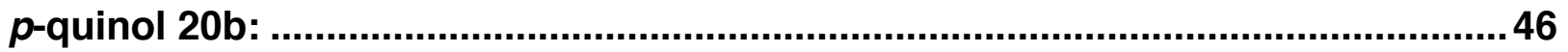

Tricycle 21:

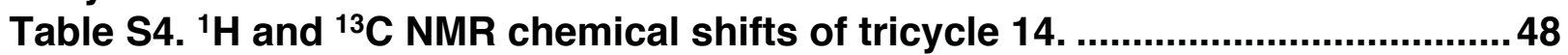

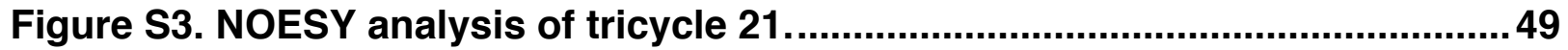

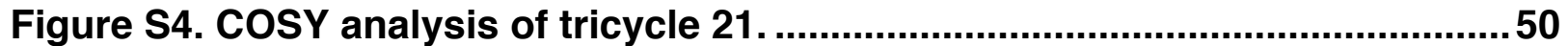

Table S5: Relative energies of tricycles 21, 21', and epi-1...............................51

Table S6. Examination of other protected substrates for HAT Hydrogenation ....52

Experimental Procedures for the Formal Enantioselective Total Synthesis of (-)-1

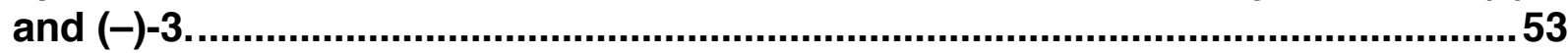

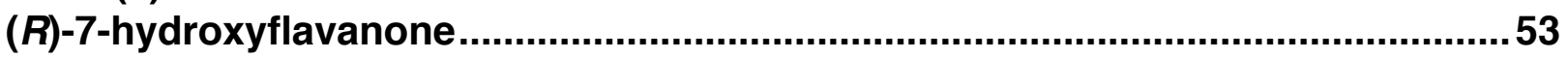

Figure S5. Chiral HPLC traces of racemic 5 and enantioenriched $(R)-5 \ldots \ldots \ldots \ldots . . . .54$

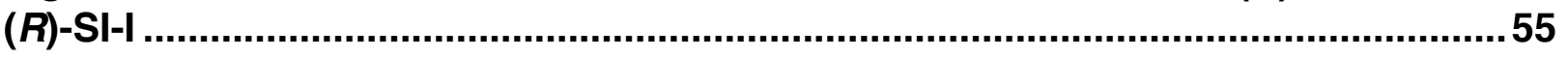

Figure S6: Chiral HPLC traces of racemic SI-I and enantioenriched SI-I ..............56

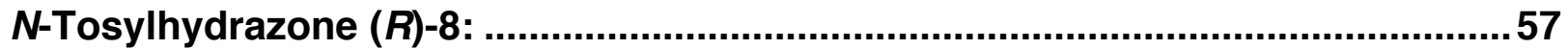

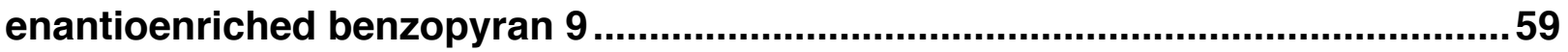

Figure S8: Chiral HPLC traces of racemic 9 and enantioenriched 9.....................60

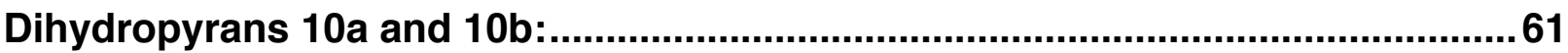

Figure S9: Chiral HPLC traces of racemic 10a and enantioenriched 10a and

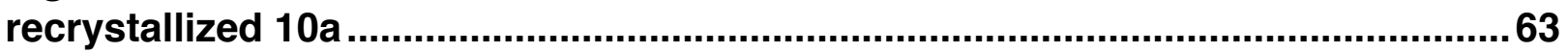

Figure S10: Chiral HPLC traces of racemic 10b and enantioenriched 10a. ..........64

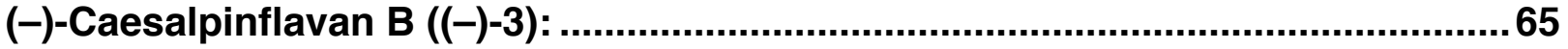

Figure S11: Chiral UPLC traces of racemic $(-)-3$ and racemic 3. .........................66

NMR Spectra of New Compounds ......................................................67

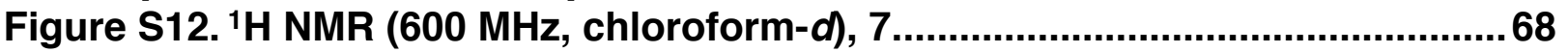

Figure S13. ${ }^{1} \mathrm{H}$ NMR (600 MHz, chloroform-d), 7 (inset). .........................................69

Figure S14. ${ }^{1} \mathrm{H}$ NMR (600 MHz, chloroform-d), 7 (inset) .........................................70

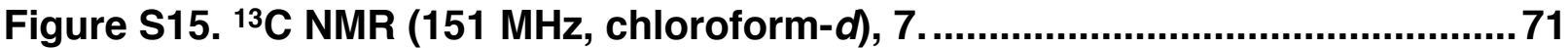

Figure S16, ${ }^{13} \mathrm{C}$ NMR (151 MHz, chloroform- $d$ ), 7 (inset). .......................................72

Figure S17. ${ }^{13} \mathrm{C}$ NMR (151 MHz, chloroform- $d$ ), 7 (inset). ........................................73 


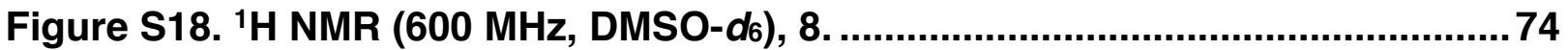

Figure S19. ${ }^{1} \mathrm{H}$ NMR (600 MHz, DMSO-d $)$, 8 (inset). ..............................................

Figure S20. ${ }^{1} \mathrm{H}$ NMR (600 MHz, DMSO- $\left.d_{6}\right)$, 8 (inset). ...........................................76

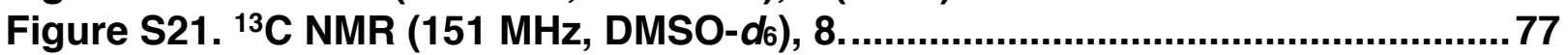

Figure S22. ${ }^{13} \mathrm{C}$ NMR (151 MHz, DMSO-d6), 8 (inset) ............................................78

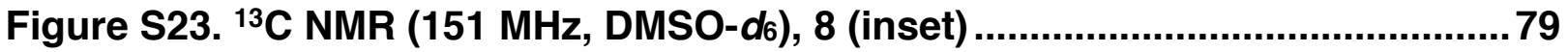

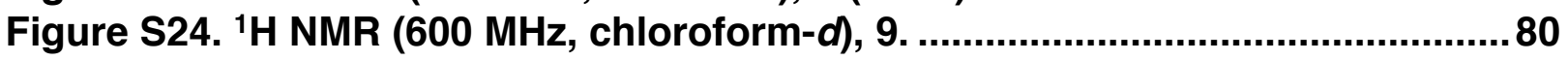

Figure S25. ${ }^{1} \mathrm{H}$ NMR (600 MHz, chloroform-d), 9 (inset). .......................................... 81

Figure S26. ${ }^{1} \mathrm{H}$ NMR (600 MHz, chloroform- $d$ ), 9 (inset) ........................................ 82

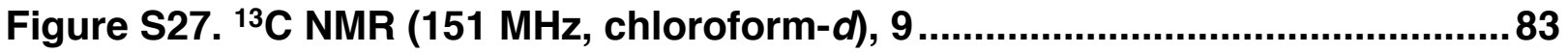

Figure S28. ${ }^{13} \mathrm{C}$ NMR (151 MHz, chloroform- $d$ ), 9 (inset). ......................................... 84

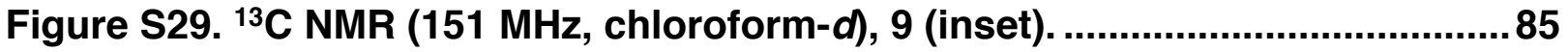

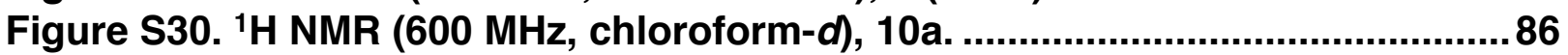

Figure S31. ${ }^{1} \mathrm{H}$ NMR $(600 \mathrm{MHz}$, chloroform- $d$ ), 10a (inset) ...................................8 87

Figure S32. ${ }^{1} \mathrm{H}$ NMR $(600 \mathrm{MHz}$, chloroform- $d)$, 10a (inset) .......................................8 88

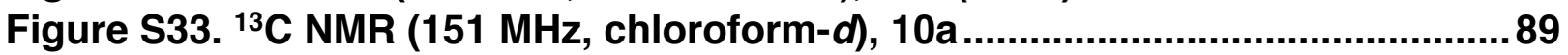

Figure S34. ${ }^{13} \mathrm{C}$ NMR (151 MHz, chloroform- $d$ ), 10a (inset)....................................90

Figure S35. ${ }^{13} \mathrm{C}$ NMR (151 MHz, chloroform- $d$ ), 10a (inset) ......................................91

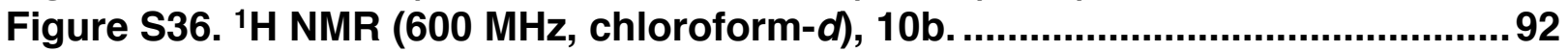

Figure S37. ${ }^{1} \mathrm{H}$ NMR (600 MHz, chloroform- $d$ ), 10b (inset). ...................................93

Figure S38. ${ }^{1} \mathrm{H}$ NMR (600 MHz, chloroform- $d$ ), 10b (inset) ....................................99

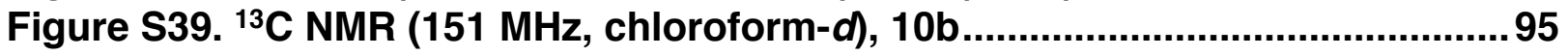

Figure S40. ${ }^{13} \mathrm{C}$ NMR (151 MHz, chloroform- $d$ ), 10b (inset)......................................96

Figure S41. ${ }^{13} \mathrm{C}$ NMR (151 MHz, chloroform-d), 10b (inset)..................................97

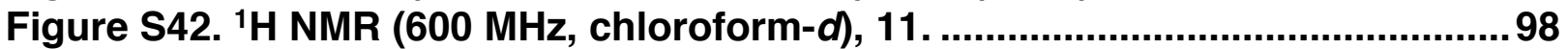

Figure S43. ${ }^{1} \mathrm{H}$ NMR (600 MHz, chloroform-d), 11 (inset). ...................................99

Figure S44. ${ }^{1} \mathrm{H}$ NMR $(600 \mathrm{MHz}$, chloroform- $d$ ), 11 (inset). .................................100

Figure S46. ${ }^{13} \mathrm{C}$ NMR (151 MHz, chloroform- $d$ ), 11 (inset). ..................................102

Figure S47. ${ }^{13} \mathrm{C}$ NMR (151 MHz, chloroform-d), 11 (inset). ..................................103

Figure S48. ${ }^{13} \mathrm{C}$ NMR (600 MHz, chloroform- $\left.d\right)$, 12 ............................................104

Figure S49. ${ }^{1} \mathrm{H}$ NMR (600 MHz, chloroform- $d$ ), 12 (inset). ...................................105

Figure S51. ${ }^{3} \mathrm{C}$ NMR (151 MHz, chloroform- $\left.d\right)$, 12. ...........................................107

Figure S52. ${ }^{3} \mathrm{C}$ NMR (151 MHz, chloroform- $d$ ), 12 (inset) ....................................108

Figure S53. ${ }^{3} \mathrm{C}$ NMR (151 MHz, chloroform-d), 12 (inset). ..................................109

Figure S54. ${ }^{1} \mathrm{H}$ NMR (600 MHz, chloroform- $d$ ), 13 (crude spectrum). ...................110

Figure S55. ${ }^{13} \mathrm{C}$ NMR (151 MHz, chloroform- $d$ ), 13 (crude spectrum). .................111

Figure S57 ${ }^{1} \mathrm{H}$ NMR (600 MHz, chloroform-d), 14 (insert). ..................................113

Figure S59. ${ }^{13} \mathrm{C}$ NMR (151 MHz, chloroform-d), 14 (insert)................................115

Figure S61. ${ }^{1} \mathrm{H}$ NMR $(600 \mathrm{MHz}$, chloroform- $d)$, 14, less polar atropisomer..........117

Figure S62. ${ }^{1} \mathrm{H}$ NMR (600 MHz, chloroform- $\left.d\right)$, 14, more polar atropisomer ........118

Figure S63. COSY NMR spectrum, 14, less polar diastereomer..........................119

Figure S64. COSY NMR spectrum, 14, more polar diastereomer. .......................120

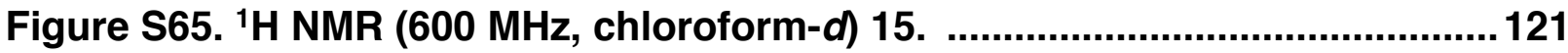

Figure S66. ${ }^{1} \mathrm{H}$ NMR (600 MHz, chloroform-d) 15 (inset). .....................................122 
Figure S67. ${ }^{1} \mathrm{H}$ NMR (600 MHz, chloroform-d) 15 (inset). ...................................123

Figure S68. ${ }^{13} \mathrm{C}$ NMR (151 MHz, chloroform-d) 15. ..........................................124

Figure S69. ${ }^{13} \mathrm{C}$ NMR (151 MHz, chloroform-d) 15 (inset)..................................125

Figure S70. ${ }^{13} \mathrm{C}$ NMR (151 MHz, chloroform-d) 15 (inset) .....................................126

Figure S71. ${ }^{1} \mathrm{H}$ NMR (600 MHz, chloroform- $\left.d\right)$ 16. ............................................12

Figure S72. ${ }^{1} \mathrm{H}$ NMR (600 MHz, chloroform- $\left.d\right) 16$ (inset). .....................................128

Figure S73. ${ }^{1} \mathrm{H}$ NMR (600 MHz, chloroform-d) 16 (inset). ....................................129

Figure S74. ${ }^{13} \mathrm{C}$ NMR (151 MHz, chloroform-d) 16 ..........................................130

Figure S75. ${ }^{13} \mathrm{C}$ NMR (151 MHz, chloroform-d) 16 (inset) ....................................131

Figure S76. ${ }^{13} \mathrm{C}$ NMR (151 MHz, chloroform-d) 16 (inset) .....................................132

Figure S77. ${ }^{1} \mathrm{H}$ NMR (600 MHZ, chloroform-d) 17.............................................133

Figure S78. ${ }^{1} \mathrm{H}$ NMR (600 MHZ, chloroform-d) 17 (inset) ...................................134

Figure S79. ${ }^{1} \mathrm{H}$ NMR (600 MHZ, chloroform-d) 17 (inset) ..................................135

Figure S80. ${ }^{13} \mathrm{C}$ NMR (151 MHz, chloroform-d) 17..........................................136

Figure S81. ${ }^{13} \mathrm{C}$ NMR (151 MHz, chloroform-d) 17 (inset) ...................................137

Figure S82. ${ }^{13} \mathrm{C}$ NMR (151 MHz, chloroform-d) 17 (inset).................................138

Figure S83. ${ }^{1} \mathrm{H}$ NMR (600 MHz, chloroform- $d$ ), 18a ..........................................139

Figure S84. Variable temperature ${ }^{1} \mathrm{H}$ NMR of $18 \mathrm{a}\left(400 \mathrm{MHz}, \mathrm{PhMe}-d_{8}\right)$ from 300 to

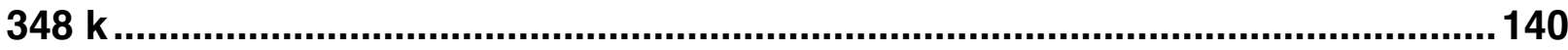

Figure S85. ${ }^{1} \mathrm{H}$ NMR $\left(600 \mathrm{MHz}\right.$, methanol- $\left.d_{4}\right)$, Caesalpinflavan B (3) ...................141

Figure S86. ${ }^{1} \mathrm{H}$ NMR $\left(600 \mathrm{MHz}\right.$, methanol- $\left.d_{4}\right)$, Caesalpinflavan B (3) (inset)........ 142

Figure S87. ${ }^{1} \mathrm{H}$ NMR $\left(600 \mathrm{MHz}\right.$, methanol- $\left.d_{4}\right)$, Caesalpinflavan B (3) (inset)........ 143

Figure S88. ${ }^{1} \mathrm{H}$ NMR (151 MHz, methanol- $\left.d_{4}\right)$, Caesalpinflavan B (3) .................. 144

Figure S89. ${ }^{1} \mathrm{H}$ NMR (151 MHz, methanol- $\left.d_{4}\right)$, Caesalpinflavan B (3) (inset)........ 145

Figure S90. ${ }^{1} \mathrm{H}$ NMR (151 MHz, methanol- $\left.d_{4}\right)$, Caesalpinflavan B (3) (inset)........ 146

Figure S91. ${ }^{1} \mathrm{H}$ NMR (600 MHz, chloroform- $\left.d\right)$, 19a ..........................................147

Figure S92. ${ }^{1} \mathrm{H}$ NMR (600 MHz, chloroform-d), 19a (inser) .................................148

Figure S93. ${ }^{1} \mathrm{H}$ NMR $(600 \mathrm{MHz}$, chloroform- $d)$, 19a (inset) .................................149

Figure S94. ${ }^{13} \mathrm{C}$ NMR (151 MHz, chloroform-d), 19a.........................................150

Figure S95. ${ }^{13} \mathrm{C}$ NMR (151 MHz, chloroform- $d$ ), 19a (inset) ..................................151

Figure S96. ${ }^{13} \mathrm{C}$ NMR (151 MHz, chloroform- $d$ ), 19a (inset). ...............................152

Figure S97. ${ }^{1} \mathrm{H}$ NMR $\left(600 \mathrm{MHz}\right.$, acetone- $\left.d_{6}\right)$, Caesalpinnone A (1)......................153

Figure S98. ${ }^{1} \mathrm{H}$ NMR $\left(600 \mathrm{MHz}\right.$, acetone- $\left.d_{6}\right)$, Caesalpinnone A (1) (inset) .............154

Figure S99. ${ }^{1} \mathrm{H}$ NMR $\left(600 \mathrm{MHz}\right.$, acetone- $\left.d_{6}\right)$, Caesalpinnone A (1) (inset) ............ 155

Figure S100. ${ }^{13} \mathrm{C}$ NMR (151 MHz, acetone-d6), Caesalpinnone A (1)...................156

Figure S101. ${ }^{13} \mathrm{C}$ NMR (151 MHz, acetone- $\left.d_{6}\right)$, Caesalpinnone A (1) (inset).........157

Figure S102. ${ }^{13} \mathrm{C}$ NMR (151 MHz, acetone- $\left.d_{6}\right)$, Caesalpinnone A (1) (inset).........158

Figure S103. ${ }^{1} \mathrm{H}$ NMR (600 MHz, chloroform- $d$ ), 18b........................................159

Figure S104. ${ }^{1} \mathrm{H}$ NMR (600 MHz, chloroform- ), 18b (inset)...............................160

Figure S105. ${ }^{1} \mathrm{H}$ NMR (600 MHz, chloroform-d), 18b (inset)...............................161

Figure S106. ${ }^{13} \mathrm{C}$ NMR (151 MHz, chloroform- $d$ ), 18b.........................................162

Figure S107. ${ }^{13} \mathrm{C}$ NMR (151 MHz, chloroform- $d$ ), 18b (inset) ................................163

Figure S108. ${ }^{13} \mathrm{C}$ NMR (151 MHz, chloroform-d), 18b (inset)..............................164

Figure S109. ${ }^{1} \mathrm{H}$ NMR (600 MHz, chloroform- $d$ ), 19b ........................................... 165 
Figure S110. ${ }^{1} \mathrm{H}$ NMR (600 MHz, chloroform- $d$ ), 19b (inset) ................................. 166

Figure S111. ' $\mathrm{H}$ NMR (600 MHz, chloroform- $d$ ), 19b (inset) .................................167

Figure S112. ${ }^{13} \mathrm{C}$ NMR (151 MHz, chloroform-d), 19b.........................................168

Figure S113. ${ }^{13} \mathrm{C}$ NMR (151 MHz, chloroform-d), 19b (inset) ................................169

Figure S114. ${ }^{13} \mathrm{C}$ NMR (151 MHz, chloroform- $d$ ), 19b (inset) ...............................170

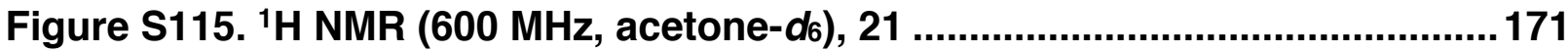

Figure S116. ${ }^{1} \mathrm{H}$ NMR $\left(600 \mathrm{MHz}\right.$, acetone- $\left.d_{6}\right), 21$ (inset) ......................................172

Figure S117. ${ }^{1} \mathrm{H}$ NMR $\left(600 \mathrm{MHz}\right.$, acetone- $\left.d_{6}\right), 21$ (inset) ...................................173

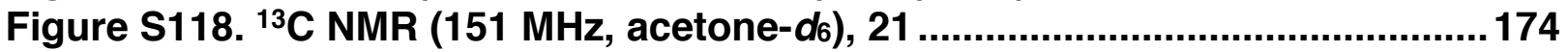

Figure S119. ${ }^{13} \mathrm{C}$ NMR (151 MHz, acetone- $\left.d_{6}\right), 21$ (inset) .....................................175

Figure S120. ${ }^{13} \mathrm{C}$ NMR (151 MHz, acetone-d6), 21 (inset) ......................................176

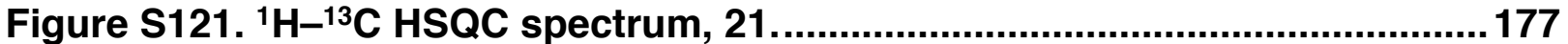

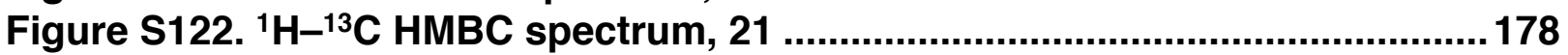

Figure S123. ${ }^{1} \mathrm{H}$ NMR (600 MHz, chloroform-d), SI-2 .......................................179

Figure S124. ${ }^{1} \mathrm{H}$ NMR (600 MHz, chloroform- $d$ ), SI-2 (inset)...............................180

Figure S125. ${ }^{1} \mathrm{H}$ NMR (600 MHz, chloroform-d), SI-2 (inset)...............................181

Figure S126. ${ }^{13} \mathrm{C}$ NMR (151 MHz, chloroform-d), SI-2 ....................................182

Figure S127. ${ }^{13} \mathrm{C}$ NMR (151 MHz, chloroform- $d$ ), SI-2 (inset) ..............................183

Figure S128. ${ }^{13} \mathrm{C}$ NMR (151 MHz, chloroform-d), SI-2 (inset) ...............................184

Figure S129. ${ }^{1} \mathrm{H}$ NMR (600 MHz, chloroform- $d$ ), SI-3.......................................... 185

Figure S130. ' $\mathrm{H}$ NMR (600 MHz, chloroform- $d$ ), SI-3 (inset)................................186

Figure S131. ${ }^{1} \mathrm{H}$ NMR (600 MHz, chloroform- $d$ ), SI-3 (inset) .................................187

Figure S132. ${ }^{13} \mathrm{C}$ NMR (151 MHz, chloroform- $d$ ), SI-3. ........................................188

Figure S133. ${ }^{13} \mathrm{C}$ NMR (151 MHz, chloroform-d), SI-3 (inset). .............................189

Figure S134. ${ }^{13} \mathrm{C}$ NMR (151 MHz, chloroform-d), SI-3 (inset). ............................190

Figure S135. ${ }^{1} \mathrm{H}$ NMR (600 MHz, chloroform- $\left.d\right)$, SI-4............................................191

Figure S136. ${ }^{1} \mathrm{H}$ NMR (600 MHz, chloroform- ), Sl-4 (inset)...............................192

Figure S137. ' $\mathrm{H}$ NMR (600 MHz, chloroform- $d$ ), SI-4 (inset)................................193

Figure S138. ${ }^{13} \mathrm{C}$ NMR (151 MHz, chloroform-d), SI-4. .......................................194

Figure S139. ${ }^{13} \mathrm{C}$ NMR (151 MHz, chloroform-d), SI-4 (inset) ................................195

Figure S140. ${ }^{13} \mathrm{C}$ NMR (151 MHz, chloroform-d), SI-4 (inset) ...............................196

Figure S141. ${ }^{1} \mathrm{H}$ NMR (600 MHz, chloroform- $d$ ), Sl-5...........................................197

Figure S142. ${ }^{1} \mathrm{H}$ NMR (600 MHz, chloroform- $d$ ), SI-5 (inset)................................198

Figure S143. ${ }^{1} \mathrm{H}$ NMR (600 MHz, chloroform- $d$ ), SI-5 (inset)..............................199

Figure S144. ${ }^{13} \mathrm{C}$ NMR (151 MHz, chloroform-d), SI-5. ........................................200

Figure S145. ${ }^{13} \mathrm{C}$ NMR (151 MHz, chloroform- $d$ ), SI-5 (inset). ...............................2.201

Figure S146. ${ }^{13} \mathrm{C}$ NMR (151 MHz, chloroform-d), SI-5 (inset). ...............................202

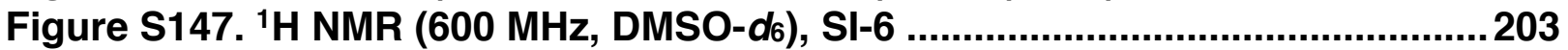

Figure S148. ${ }^{1} \mathrm{H}$ NMR (600 MHz, DMSO- $\left.d_{6}\right)$, SI-6 (inset)....................................204

Figure S149. ${ }^{1} \mathrm{H}$ NMR (600 MHz, DMSO- $d_{6}$ ), SI-6 (inset)......................................205

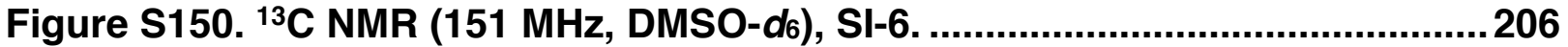

Figure S151. ${ }^{13} \mathrm{C}$ NMR (151 MHz, DMSO-d6), SI-6 (inset) ......................................207

Figure S152. ${ }^{13} \mathrm{C}$ NMR (151 MHz, DMSO-d $d_{6}$ ), SI-6 (inset) ...........................208

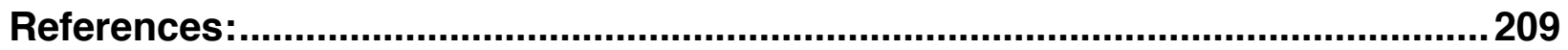




\section{General Information}

Unless otherwise noted, reactions were performed in either oven $\left(115^{\circ} \mathrm{C}\right)$ - or flame-dried glassware and under an atmosphere of nitrogen or argon employing standard Schleck techniques. Diethyl ether, tetrahydrofuran (THF), methylene chloride, and toluene were purified by passage through columns of activated alumina under nitrogen (Grubbs apparatus). All work-up and purification procedures were carried out using reagent grade solvents and reagents. XPhos Pd G3 was prepared according to published procedures. ${ }^{51}$ Flash column chromatography (FCC) was performed with 200-400 mesh silica gel (Sillicycle, P60). Medium Pressure Liquid Chromotography (MPLC) was performed on Teledyne Isco instruments (NextGen $300+$ or Lumen Rf+) and using Teledyne silica gel cartridges at the recommended flow rate. All High Performance Liquid Chromatography (HPLC) separations were performed on a Waters HPLC system (Waters 1525 binary HPLC pump with direct injection port accompanied by a Waters 2489 UVVisible detector and monitored at 254 or $280 \mathrm{~nm}$ ). Preparative HPLC separations were performed using a normal-phase Sunfire, Silica Prep $10 \mu \mathrm{m}, 10$ × $250 \mathrm{~mm}$ column equipped with guard column. All chiral separations were performed using the column specified in the report for each respective compound. All purifications utilized a normalphase Sunfire, Silica Prep $10 \mu \mathrm{m}, 10 \times 250 \mathrm{~mm}$ column. Thin layer chromatography (TLC) was performed on silica gel 60 F254 (EMD Chemicals Inc.). Reverse-Phase thin layer chromatography was performed on $\mathrm{C}_{18}$ silica gel plates with fluorescent indicator (Millipore Sigma). Room temperature is 23 or $24^{\circ} \mathrm{C}$. Infrared spectra were obtained using 
a Bruker Alpha ATR-IR. Peak frequencies are reported in $\mathrm{cm}^{-1}$ and are defined as: vs (very strong), $\mathrm{s}$ (strong), $\mathrm{m}$ (medium), w (weak), br (broad). ${ }^{1} \mathrm{H}$ and ${ }^{13} \mathrm{C}$ NMR spectra were recorded on a Bruker 400, Varian 500, or Bruker 600. Chemical shifts ( $\delta)$ are reported in parts per million (ppm) relative to internal residual solvent peaks from indicated deuterated solvents: Chloroform- $d\left({ }^{1} \mathrm{H}: 7.26 \mathrm{ppm},{ }^{13} \mathrm{C}: 77.16 \mathrm{ppm}\right)$, methanol- $d_{4}\left({ }^{1} \mathrm{H}: 3.31\right.$ ppm, $\left.{ }^{13} \mathrm{C}: 47.6 \mathrm{ppm}\right)$, acetone- $d_{6}\left({ }^{1} \mathrm{H}: 2.07 \mathrm{ppm},{ }^{13} \mathrm{C}: 29.0 \mathrm{ppm}\right)$. Coupling constants $(\mathcal{J}$ are reported in Hertz $(\mathrm{Hz})$ and are rounded to the nearest $0.1 \mathrm{~Hz}$. Resonance multiplicities are defined as: $\mathrm{s}=$ singlet, $\mathrm{bs}=$ broad singlet, $\mathrm{d}=$ doublet, $\mathrm{t}=$ triplet, $\mathrm{q}=$ quartet, $\mathrm{m}=$ multiplet, $\mathrm{dd}=$ doublet of doublets, $\mathrm{dt}=$ doublet of triplets, $\mathrm{ddd}=$ doublet of doublet of doublets, at = apparent triplet. High Resolution Mass (HRMS) analysis was obtained using electro-spray ionization (ESI) were obtained on a ThermoScientific LTQ Orbitrap and the found mass represents an average of three, individual, direct infusions.

\section{Reagents}

All reagents were procured as described below and were $>95 \%$ purity grade, except where noted.

Acetic Anhydride was purchased from Acros and used as received.

Allyl Bromide was purchased from Millipore Sigma and used as received.

AIBN was purchased from Acros and used as received.

Ammonium hexafluorophosphate was purchased from Sigma-Aldrich and used as received.

Benzaldehyde was purchased from Millipore Sigma and distilled prior to use. 
Boron tribromide diethyl etherate was purchased from Millipore Sigma and used as received.

Borane tert-butyl amine complex was purchased from Fluka and used as recieved.

3-chloroperbenzoic acid was purchased from Millipore Sigma and used as received.

Carbon Disulfide was purchased from Millipore Sigma and used as received.

Dichloroethane (anhydrous) was purchased from Millipore Sigma and used as received 4-(dimethylamino)pyridine (DMAP) was purchased from Millipore Sigma and used as received.

1,3-dimethylbarbituric acid was purchased from Millipore sigma and used as received. Ethylene glycol was purchased from Acros and used as received.

Fluorobenzene was purchased from Oakwood and dried over activated $3 \mathrm{~A}$ molecular sieves and sparged with dry argon prior to use.

7-hydroxyflavanone was purchased from TCI Chemical and used as received.

Methyl lodide was purchased form Oakwood and used as received.

Palladium bis(trifluoroacetate) was purchased from Millipore Sigma and used as received.

Potassium Carbonate was purchased from Fisher Scientific

Phenylboronic acid was purchased from Acros and used as received.

Phenylsilane was purchased from Millipore Sigma and used as received.

Pyridine was purchased from Oakwood and distilled over $\mathrm{CaH}_{2}$ prior to use.

4-Toluenesulfonic acid monohydrate was purchased from Millipore Sigma and used as received. 
Tert-butylhydroperoxide (5.5 $\mathrm{M}$ in decane) was purchased from Millipore Sigma and stored over activated 3A molecular sieves prior to use.

$\mathbf{N}$-Tosylhydrazide was purchased from Millipore Sigma or Oakwood Chemical and used as received.

Tetrakis (triphenylphosphine) palladium was purchased from Strem Chemical and used as received.

Tetra-n-butyl tin hydride was purchased from Oakwood and used as received Tris(dipivaloylmethane) Manganese (III) $\left(\mathrm{Mn}(\mathrm{dpm})_{3}\right)$ was purchased from Strem Chemical and used as received.

Trifluorotoluene (anhydrous) was purchased from Aldrich and used as received.

Palladium hydroxide on activated carbon (20 wt \%) (Pearlman's catalyst) was purchased from Millipore Sigma and used as received.

Phenyliodide diacetate (PIDA) was purchased from Millipore Sigma and used as received.

XPhos was purchased from Strem Chemicals.

\section{Experimental Procedures:}


<smiles>O=C1CC(c2ccccc2)Oc2cc(O)ccc21</smiles>

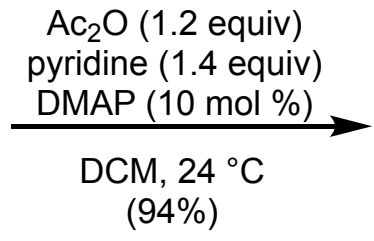<smiles>CC(=O)Oc1ccc2c(c1)OC(c1ccccc1)CC2=O</smiles>

\section{Racemic acetate SI-I}

To a solution of racemic 7-hydroxyflavanone $(2.50 \mathrm{~g}, 10.4 \mathrm{mmol}, 1$ equiv) in DCM $(80 \mathrm{~mL})$ was added successively acetic anhydride $(1.3 \mathrm{~mL}, 12.5 \mathrm{mmol}, 1.2$ equiv), pyridine (1.3 mL, $15.6 \mathrm{mmol}, 1.5$ equiv), and DMAP (150 mg, $1.2 \mathrm{mmol})$. The reaction was stirred for 90 minutes before silica gel (ca. $4 \mathrm{~g}$ ) was added and the volatiles removed in vacuo before being directly loaded in to an empty MPLC cartridge $(25 \mathrm{~g})$ and chromatographed ( $80 \mathrm{~g}$ column, $60 \mathrm{~mL} / \mathrm{min}, 10$--> $40 \%$ ethyl acetate-hexanes) to give acetate $5(2.75 \mathrm{~g}, 94 \%)$ as a colorless microcrystalline solid. The spectral data of SI-I matched those previously reported. ${ }^{\mathrm{S}}$

${ }^{1} \mathrm{H}$ NMR (400 MHz, $\left.\mathrm{CDCl}_{3}\right): \delta 7.95(\mathrm{~d}, J=8.5 \mathrm{~Hz}, 1 \mathrm{H}), 7.51-7.34(\mathrm{~m}, 5 \mathrm{H}), 6.84(\mathrm{~d}, J=$ $2.1 \mathrm{~Hz}, 1 \mathrm{H}), 6.80(\mathrm{dd}, J=8.6,2.1 \mathrm{~Hz}, 1 \mathrm{H}), 5.49(\mathrm{dd}, J=13.3,2.9 \mathrm{~Hz}, 1 \mathrm{H}), 3.06(\mathrm{dd}, J=$ 16.9, 13.3 Hz, 1H), 2.87 (dd, $J=16.9,3.0 \mathrm{~Hz}, 1 \mathrm{H}$ ), 2.30 (s, 3H). 

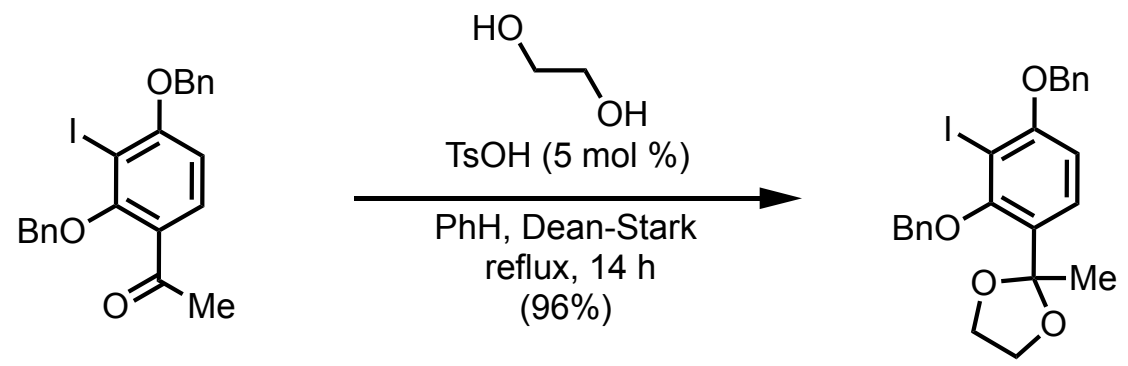

\section{Aryl lodide 7}

To a $1 \mathrm{~L}$ single-necked round-bottom flask containing a magnetic stir-bar was added 2,4-bis(benzyloxy)-3-iodoacetopenone ${ }^{\mathrm{S3}}$ (13.8 g, $30.0 \mathrm{mmol}, 1$ equiv), $p$ toluenesulfonic acid monohydrate (282 $\mathrm{mg}, 1.5 \mathrm{mmol}, 5 \mathrm{~mol} \%)$ and dry benzene (210 $\mathrm{mL})$. To this stirred solution was added ethylene glycol $(8.5 \mathrm{~mL}, 120 \mathrm{mmol}, 4.0$ equiv). The flask was then equipped with a Dean-Stark trap $(25 \mathrm{~mL}$ reservoir filled with dry benzene) and reflux condenser. The apparatus was purged with an atmosphere of argon, left under a static atmosphere of argon and heated to $105^{\circ} \mathrm{C}$ for 14 hours. The flask was then cooled to $4{ }^{\circ} \mathrm{C}$ and a chilled solution of saturated $\mathrm{NaHCO}_{3}$ was added with vigorous stirring. The suspension was transferred to a $2 \mathrm{~L}$ separatory funnel and the organic and aqueous layers separated. The aqueous layer was washed with EtOAc $(3 \times 150 \mathrm{~mL})$ and the combined organic layers washed with saturated brine $(1 \times 200 \mathrm{~mL})$. The combined organic layers were then dried over $\mathrm{Na}_{2} \mathrm{SO}_{4}$ and concentrated in vacuo to afford 7 (14.5 $\mathrm{g}, 96 \%$ ) as a light-pink microcrystalline solid (Notes 1 and 2).

Note 1: 7 was a typically afforded in $>95 \%$ purity $\left({ }^{1} \mathrm{H}\right.$ NMR), but if further purification was needed, it was accomplished by recrystallization from 10:1 hexanes-PhMe to yield analytically pure 7 .

Note 2: Attempts to purify 7 by silica gel chromatography led to poorly resolved mixtures of 2,4-bis(benzyloxy)-3-iodoacetopenone and 7, presumably from deprotection of the labile aryl acetal on silica gel.

1H NMR: $\left(600 \mathrm{MHz}, \mathrm{CDCl}_{3}\right): \delta 7.74(\mathrm{~d}, J=7.8 \mathrm{~Hz}, 2 \mathrm{H}), 7.65-7.32(\mathrm{~m}, 9 \mathrm{H}), 6.67(\mathrm{dd}, \mathrm{J}$ $=8.5,1.3,1 \mathrm{H}), 5.20(\mathrm{~s}, 2 \mathrm{H}), 5.18(\mathrm{~s}, 2 \mathrm{H}), 4.25-4.05(\mathrm{~m}, 2 \mathrm{H}), 3.98-3.84(\mathrm{~m}, 2 \mathrm{H})$, 1.83 (s, $3 \mathrm{H}) .{ }^{13} \mathrm{C}$ NMR (151 MHz, $\left.\mathrm{CDCl}_{3}\right): \delta$ 158.6, 157.4, 137.5, 135.4, 130.3, 128.7, 128.5, 128.3, 128.0, 127.9, 127.9, 127.0, 106.3, 107.6, 87.6, 75.3, 71.09, 64.49, 26.2. HRMS (ESI+): Calcd. for $\mathrm{C}_{24} \mathrm{H}_{23} \mathrm{IO}_{4} \mathrm{Na}$. [M+Na] ${ }^{+}: 525.0529$. Found: 525.0530 . IR (ATR): $v_{\max }\left(\mathrm{cm}^{-1}\right)=3064(\mathrm{w}), 3031(\mathrm{w}), 2985(\mathrm{~m}), 2377(\mathrm{w}), 1671(\mathrm{w}), 1585(\mathrm{~m}), 1497(\mathrm{~m}), 1388$ (s), $1251(\mathrm{~m}), 1198(\mathrm{~s}), 1148(\mathrm{w}), 1067(\mathrm{~m}), 1041(\mathrm{~s}), 950(\mathrm{~m}), 886(\mathrm{w}), 762(\mathrm{w}), 735(\mathrm{~s})$, 674 (s), 599 (w). TLC (EtOAc-hexanes-Et $3 \mathrm{~N}=10: 90: 1): R_{\mathrm{f}}=0.26$. 
<smiles>CC(=O)Oc1ccc2c(c1)OC(c1ccccc1)CC2=O</smiles>

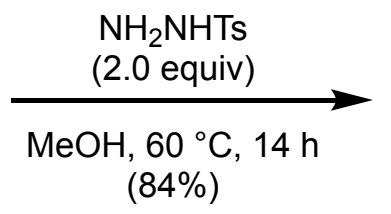<smiles>CC(=O)Oc1ccc2c(c1)OC(c1ccccc1)CC2=N[AsH2-]</smiles>

\section{Racemic $\mathbf{N}$-Tosylhydrazone 8}

To a $250 \mathrm{~mL}$ round-bottom flask equipped with a magnetic stir-bar was added SI-1 (2.75 g, $9.75 \mathrm{mmol}, 1$ equiv) and $\mathrm{MeOH}(120 \mathrm{~mL})$. To the stirred solution was added $p$ tosylhydrazide ( $3.60 \mathrm{~g}, 19.7 \mathrm{mmol}, 2.0$ equiv) and the flask then heated to $60^{\circ} \mathrm{C}$ under a static atmosphere of nitrogen. After $14 \mathrm{~h}$, the solution slowly cooled to $4{ }^{\circ} \mathrm{C}$ over the course of 1 hour. The precipitate was collected in a large Kiriyama filter to yield 8 ( $3.41 \mathrm{~g}$ ) as a colorless microcrystalline solid. The filtrate was evaporated to ca. half volume and cooled to $4 \mathrm{C}$ for 1 hour before being re-filtered to give additional $8(261 \mathrm{mg}, 3.67 \mathrm{~g}$ total, $84 \%)$. The material was azeotropically-dried from benzene $(3 \times 100 \mathrm{~mL})$ before being used in the next step.

${ }^{1} \mathrm{H}$ NMR (600 MHz, DMSO-d6) $\delta 10.63-10.55(\mathrm{~m}, 1 \mathrm{H}), 7.82(\mathrm{dd}, J=8.2,1.4 \mathrm{~Hz}, 2 \mathrm{H})$, $7.79-7.74(\mathrm{~m}, 1 \mathrm{H}), 7.54-7.47(\mathrm{~m}, 2 \mathrm{H}), 7.42$ (ddd, $J=8.8,6.1,1.2 \mathrm{~Hz}, 4 \mathrm{H}), 7.40-7.36$ $(\mathrm{m}, 1 \mathrm{H}), 6.83-6.76(\mathrm{~m}, 2 \mathrm{H}), 5.25(\mathrm{dd}, J=12.0,3.1 \mathrm{~Hz}, 1 \mathrm{H}), 3.22-3.18(\mathrm{~m}, 1 \mathrm{H}), 2.74$ (dd, $J=17.1,12.0 \mathrm{~Hz}, 1 \mathrm{H}), 2.38(\mathrm{~s}, 3 \mathrm{H}), 2.25(\mathrm{~s}, 3 \mathrm{H}) .{ }^{13} \mathrm{C}$ NMR (151 MHz, DMSO-d6) $\delta$ 168.7, 157.1, 152.6, 146.6, 143.4, 139.3, 136.0, 129.5, 128.4, 128.3, 127.5, 126.3, 125.0, 117.2, 115.6, 111.0, 76.5, 32.0, 21.0, 20.8. IR (ATR): $v_{\max }\left(\mathrm{cm}^{-1}\right)=3214(\mathrm{~m}), 1762(\mathrm{~s})$, $1623(\mathrm{~m}), 1600(\mathrm{~m}), 1433(\mathrm{~s}), 1340(\mathrm{~m}), 1205(\mathrm{~s}), 1167(\mathrm{~s}), 1082(\mathrm{~m}), 913(\mathrm{~m}), 739(\mathrm{~m})$, $699(\mathrm{~m}), 567(\mathrm{~m})$. HRMS (ESI+) Calcd. for $\mathrm{C}_{24} \mathrm{H}_{22} \mathrm{~N}_{2} \mathrm{O}_{5} \mathrm{SNa}$. [M+Na] $]^{+}: 473.1147$, found: 473.1141 


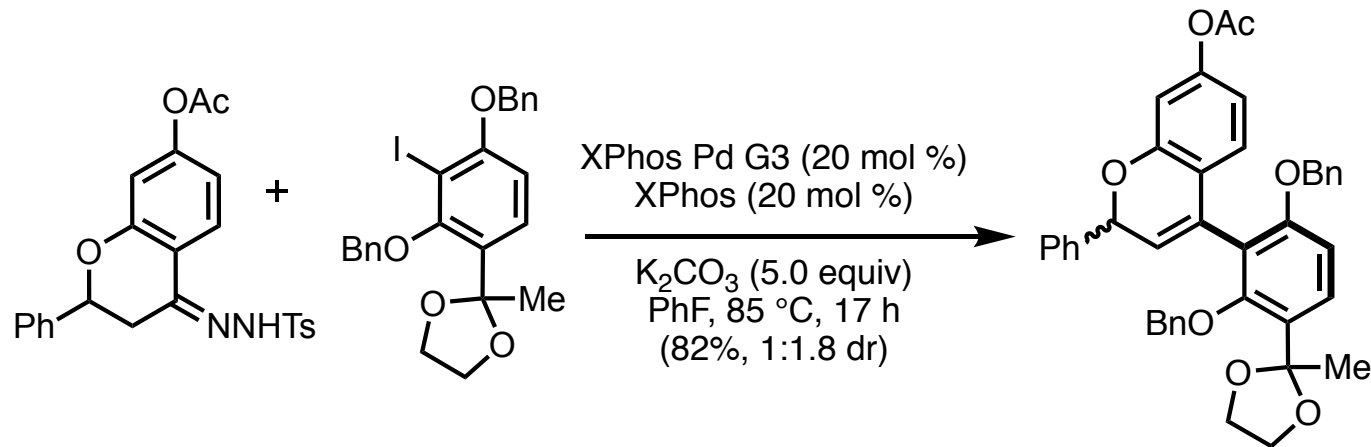

\section{Racemic Benzopyran 9}

Representative large-scale coupling reaction (with slow addition of 8):

A dry $100 \mathrm{~mL}$ round-bottomed flask was charged with 7 (398 $\mathrm{mg}, 0.79 \mathrm{mmol}, 1$ equiv), XPhos Pd G3 (140 mg, $0.16 \mathrm{mmol}, 20 \mathrm{~mol} \%$ ), XPhos (76 mg, $0.16 \mathrm{mmol}, 20 \mathrm{~mol} \%$ ), and $\mathrm{K}_{2} \mathrm{CO}_{3}$ (552 mg, $4.0 \mathrm{mmol}, 5$ equiv), followed by dry PhF (16 mL). The flask was sealed and resulting suspension sparged with argon for 15 minutes before being left under a static atmosphere of argon. The flask was then warmed to $85^{\circ} \mathrm{C}$. To a separate, dry $50 \mathrm{~mL}$ Erlenmeyer flask equipped with a 14/20 ground-glass joint was added 8 (900 $\mathrm{mg}, 2.0 \mathrm{mmol}, 2.5$ equiv). The flask was then sealed and purged with an atmosphere of argon before 1,4-dioxane $(23 \mathrm{~mL})$ was added via syringe. The resulting suspension was swirled until complete dissolution (ca. 15 minutes) and subsequently sparged with dry argon. The solution of 8 was then added slowly (ca. $2.3 \mathrm{~mL} / \mathrm{h}$ ) to the suspension containing 7 at $85^{\circ} \mathrm{C}$. After the addition was complete, the reaction let to stir for 7 hours before being cooled to $24^{\circ} \mathrm{C}$, filtered over a pad of silica gel. The silica gel pad was washed with EtOAc $(5 \times 30 \mathrm{~mL})$ and the washes combined with the filtrate before being concentrated in vacuo to give a sticky orange oil. The oil was chromatographed (MPLC, $40 \mathrm{~g}$ column, $30 \mathrm{~mL} / \mathrm{min} 10 \% \rightarrow 40 \%$ EtOAc/hexanes to give 9 as a light-yellow foam (425 mg, 82\%, 1:1.8 mixture of atropisomers) (Note 1).

Note 1: If the reaction stalls, as judged by removing an aliquot and analyzing by ${ }^{1} \mathrm{H}$ NMR, another portion of $\mathrm{K}_{2} \mathrm{CO}_{3}$ typically restarts forward progress.

Representative gram-scale coupling (without slow addition of 8):

A dry $250 \mathrm{~mL}$ round-bottomed flask was charged with a stir-bar, $7(2.78 \mathrm{~g}, 5.5 \mathrm{mmol}, 2.5$ equiv), 8 (1.02 g, $2.22 \mathrm{mmol}, 1.0$ equiv) XPhos Pd G3 (384 mg, $0.456 \mathrm{mmol}, 20 \mathrm{~mol} \%$ ), XPhos (211 mg, $0.443 \mathrm{mmol}, 20 \mathrm{~mol} \%$ ), and $\mathrm{K}_{2} \mathrm{CO}_{3}$ (939 mg, $6.66 \mathrm{mmol}, 3$ equiv), followed by dry $\mathrm{PhF}(84 \mathrm{~mL})$. The flask was sealed before being left under a static atmosphere of argon and heated to $85^{\circ} \mathrm{C}$ for 22 hours with vigorous stirring. After the reaction was complete, it was cooled to $24{ }^{\circ} \mathrm{C}$ and filtered over a pad of silica gel. The silica gel pad was washed with EtOAc $(5 \times 150 \mathrm{~mL})$ and the washes combined with the filtrate before being concentrated in vacuo to give an orange oil. The oil was 
chromatographed (MPLC, $80 \mathrm{~g}$ column, $60 \mathrm{~mL} / \mathrm{min}$ hexanes $\rightarrow 40 \%$ EtOAc/hexanes to give 9 as a light-yellow foam (970 mg, 68\%).

${ }^{1} \mathrm{H}$ NMR $\left(600 \mathrm{MHz}, \mathrm{CDCl}_{3}\right.$, both atropisomers): $\delta 7.56-7.54(\mathrm{~m}, 2.9), 7.41-7.40(\mathrm{~m}$, $7.4 \mathrm{H}), 7.38-7.35(\mathrm{~m}, 3.9 \mathrm{H}), 7.33-7.31(\mathrm{~m}, 5.9 \mathrm{H}), 7.29-7.26(\mathrm{~m}, 9.9 \mathrm{H}$, overlap with $\left.\mathrm{CHCl}_{3}\right), 7.24-7.18(\mathrm{~m}, 6.9 \mathrm{H}), 7.16-7.10(\mathrm{~m}, 9.1 \mathrm{H}), 6.90(\mathrm{~d}, J=8.3 \mathrm{~Hz}, 1.8 \mathrm{H}), 6.85(\mathrm{~d}$, $J=8.4 \mathrm{~Hz}, 1.0 \mathrm{H}), 6.81-6.77(\mathrm{~m}, 2.8 \mathrm{H}), 6.72(\mathrm{~d}, J=2.2 \mathrm{~Hz}, 1.0 \mathrm{H}), 6.63(\mathrm{~d}, J=2.2 \mathrm{~Hz}$, $1.7 \mathrm{H}), 6.60-6.58(\mathrm{~m}, 2.8 \mathrm{H}), 6.06(\mathrm{~d}, J=3.3 \mathrm{~Hz}, 1.0 \mathrm{H}), 5.85(\mathrm{~d}, J=3.4 \mathrm{~Hz}, 1 \mathrm{H}), 5.80(\mathrm{~d}$, $J=3.5 \mathrm{~Hz}, 1.7 \mathrm{H}), 5.77(\mathrm{~d}, J=3.6 \mathrm{~Hz}, 1.8 \mathrm{H}), 5.06-5.03(\mathrm{~m}, 5.7 \mathrm{H}), 4.98(\mathrm{~d}, J=10.0 \mathrm{~Hz}$, $1.8 \mathrm{H}), 4.88(\mathrm{~d}, J=10.3 \mathrm{~Hz}, 1.0 \mathrm{H}), 4.78(\mathrm{~d}, J=10.3 \mathrm{~Hz}, 1.0 \mathrm{H}), 4.74(\mathrm{~d}, J=10.0 \mathrm{~Hz}, 1.8 \mathrm{H})$, $4.14-4.04(\mathrm{~m}, 5.7 \mathrm{H}), 3.99-3.96(\mathrm{~m}, 1.8 \mathrm{H}), 3.94-3.87(3.9 \mathrm{H}), 2.29(\mathrm{~s}, 3.1 \mathrm{H}), 2.26(\mathrm{~s}$, $5.3 \mathrm{H}), 1.83-1.80(\mathrm{~m}, 8.2 \mathrm{H}) .{ }^{13} \mathrm{C} \mathrm{NMR}\left(151 \mathrm{MHz}, \mathrm{CDCl}_{3}\right.$, both atropisomers): $\delta 169.2$, $169.1,157.5,157.5,156.2,155.9,154.4,153.9,151.2,140.7,139.9,138.0,138.0,137.0$, 136.7, 129.4, 128.6, 128.5, 128.5, 128.5, 128.4, 128.4, 128.3, 128.3, 128.2, 128.2, 128.2, 127.8, 127.8, 127.7, 127.7, 127.7, 127.3, 127.3, 127.2, 127.0, 126.8, 126.0, 125.8, 125.5, 125.3, 122.5, 122.1, 120.8, 120.6, 114.1, 114.0, 109.9, 109.7, 108.9, 108.8, 107.5, 106.9, $77.7,77.5,76.0,75.6,70.4,70.3,64.6,64.5,64.5,26.4,26.4,21.3$. (8 overlapping ${ }^{13} \mathrm{C}$ resonances) IR (ATR): $v_{\max }\left(\mathrm{cm}^{-1}\right)=3031(\mathrm{w}), 2933(\mathrm{~m}), 2884(\mathrm{~m}), 2359(\mathrm{w}), 1762(\mathrm{~s})$, $1608(\mathrm{~m}), 1589(\mathrm{~m}), 1465(\mathrm{~m}), 1454(\mathrm{~m}), 1371(\mathrm{~m}), 1201(\mathrm{~s}), 1143(\mathrm{~m}), 1129(\mathrm{~m}), 1072$ $(\mathrm{m}), 1043(\mathrm{~m}), 811(\mathrm{~m}), 737(\mathrm{~m}), 693(\mathrm{~m})$. HRMS (ESI+) Calcd. for $\mathrm{C}_{41} \mathrm{H}_{36} \mathrm{O}_{7} \mathrm{Na}$. [M+Na] ${ }^{+}$: 663.2359, found: 663.2354. TLC (EtOAc-hexanes $=25: 75): R_{\mathrm{f}}=0.38$. 


\section{Benzopyran 14:}

In this study, we had accessed benzopyran 15 by an earlier synthetic route (below SI-2 $\rightarrow$ Sl-4 $\rightarrow$ 15).<smiles>O=C(CCc1ccccc1)c1ccc(O)cc1OPO</smiles>

\section{4-(3-phenylpropanoyl)-1,3-phenylene bis(2,2-dimethylpropanoate) (SI-2)}

To a dry 500-mL round-bottom flask equipped with magnetic stir-bar was added 1-(2,4-dihydroxyphenyl)-3-phenylpropan- 1-one ${ }^{\mathrm{s4}}$ (35.7 g, $147 \mathrm{mmol}, 1$ equiv). The flask was sealed with a septum and purged with an atmosphere of dry nitrogen for 15 minutes before being left under a static atmosphere of nitrogen. Dry pyridine $(200 \mathrm{~mL})$ was added and the flask subsequently cooled to $4{ }^{\circ} \mathrm{C}$ in an ice bath. To the cooled solution was added pivaloyl chloride $(46.0 \mathrm{~mL}, 368 \mathrm{mmol}, 2.5$ equiv) dropwise over 15 minutes. After the addition was complete, the reaction warmed to $50{ }^{\circ} \mathrm{C}$ and stirred for 16 hours. At this point, additional pivaloyl chloride $(5.0 \mathrm{~mL})$ was added and the reaction continued to stir at $50{ }^{\circ} \mathrm{C}$. After 8 hours, the reaction was cooled to $4^{\circ} \mathrm{C}$ and $6 \mathrm{M} \mathrm{HCl}(200 \mathrm{~mL})$ added with vigorous stirring. The suspension was transferred to a $1 \mathrm{~L}$ separatory funnel and layers separated. The aqueous layer was washed with diethyl ether $(3 \times 150 \mathrm{~mL})$ and the combined organic washed with saturated $\mathrm{NaHCO}_{3}(3 \times 100 \mathrm{~mL})$ before being dried over $\mathrm{Na}_{2} \mathrm{SO}_{4}$ and concentrated in vacuo to give SI-2 $(59.8 \mathrm{~g}, 99 \%)$ as a light-yellow oil, which was used in the next step without further purification.

1H NMR: $\left(600 \mathrm{MHz}, \mathrm{CDCl}_{3}\right): \delta 7.69(\mathrm{~d}, \mathrm{~J}=8.6 \mathrm{~Hz}, 1 \mathrm{H}), 7.27-7.24(\mathrm{~m}, 2 \mathrm{H}), 7.20-7.14$ $(\mathrm{m}, 3 \mathrm{H}), 7.00$ (dd, J = 8.6 Hz, $2.3 \mathrm{~Hz}, 1 \mathrm{H}), 6.81(\mathrm{~d}, \mathrm{~J}=2.2 \mathrm{~Hz}, 1 \mathrm{H}), 3.18(\mathrm{at}, J=7.8 \mathrm{~Hz}$, $2 \mathrm{H}), 3.00$ (at, $J=7.8 \mathrm{~Hz}, 2 \mathrm{H}), 1.32(\mathrm{~s}, 9 \mathrm{H}), 1.30(\mathrm{~s}, 9 \mathrm{H}) .{ }^{13} \mathbf{C ~ N M R}\left(151 \mathrm{MHz}, \mathbf{C D C l}_{3}\right): \delta$ 197.9, 176.4, 176.0, 154.0, 150.0, 140.9, 130.1, 128.7, 128.4, 128.4, 126.0, 118.8, 117.0, 43.3, 39.1, 39.0, 29.9, 26.9, 26.9. IR (ATR): $\nu_{\max }\left(\mathrm{cm}^{-1}\right)=2974(\mathrm{~m}), 2935(\mathrm{w}), 2973(\mathrm{w})$, $2341(\mathrm{w}), 1754(\mathrm{~s}), 1691(\mathrm{~m}), 1605(\mathrm{~m}), 1479(\mathrm{~m}), 145(\mathrm{w}), 1397(\mathrm{w}), 1273(\mathrm{~m}), 1225(\mathrm{~m})$, 1089 (s), 1020 (m), 984 (w), 942 (w), 904 (w), 749 (W), 700 (w). HRMS (ESI+): Calcd. for $\mathrm{C}_{25} \mathrm{H}_{30} \mathrm{O}_{5} \mathrm{Na}$. [M+Na] $]^{+}$: 433.1991. Found: 433.1987. 
<smiles>CCCCOc1cc(O[Pb])ccc1C(=O)CCc1ccccc1</smiles><smiles>COc1ccc(C(CCc2ccccc2)=N[AsH2])c(O[PbH2])c1</smiles>

\section{4-(3-phenyl-1-(2-tosylhydrazineylidene)propyl)-1,3-phenylene bis(2,2- dimethylpropanoate) (SI-3):}

To a $2 \mathrm{~L}$ round-bottom flask equipped with a magnetic stirbar was added SI-2 (59.8 $\mathrm{g}, 146 \mathrm{mmol}, 1$ equiv) and $\mathrm{MeOH}(1.1 \mathrm{~L})$. To the stirred solution was added $p$ tosylhydrazide ( $52.7 \mathrm{~g}, 283 \mathrm{mmol}, 1.9$ equiv) and the flask then heated to $60{ }^{\circ} \mathrm{C}$ under a static atmosphere of nitrogen. After $20 \mathrm{~h}$, the solution slowly cooled to $4{ }^{\circ} \mathrm{C}$ over the course of 1 hour. The precipitate was collected in a large Kiriyama filter to yield SI-3 (33.5 g) as colorless needles. The filtrate was allowed to sit at $-20^{\circ} \mathrm{C}$ for 14 hours before the precipitate was collected to give SI-3 $(7.6 \mathrm{~g})$. This process was repeated 2 additional times and the combined crops dissolved in toluene $(250 \mathrm{~mL})$ and azeotropically-dried to eventually a total of $52.6 \mathrm{~g}(67 \%)$ of SI-3.

${ }^{1} \mathrm{H}$ NMR: $\left(600 \mathrm{MHz}, \mathrm{CDCl}_{3}\right): \delta 7.72(\mathrm{~d}, \mathrm{~J}=8.2 \mathrm{~Hz}, 2 \mathrm{H}), 7.33-7.15(\mathrm{~m}, 7 \mathrm{H}), 7.12(\mathrm{~d}, \mathrm{~J}$ $=8.4 \mathrm{~Hz}, 1 \mathrm{H}), 7.08-7.03(\mathrm{~m}, 2 \mathrm{H}), 6.95(\mathrm{dd}, \mathrm{J}=8.4,2.3 \mathrm{~Hz}, 1 \mathrm{H}), 6.89(\mathrm{~d}, \mathrm{~J}=2.3 \mathrm{~Hz}, 1$ H), $2.87-2.78(\mathrm{~m}, 2 \mathrm{H}), 2.67$ (at, J = 7.6 Hz, 2 H), 2.45 (s, 3 H), 1.37 (s, $9 \mathrm{H}), 1.23$ (s, 9 H). ${ }^{13} \mathrm{C}$ NMR (151 MHz, $\left.\mathbf{C D C l}_{3}\right): \delta$ 176.4, 176.0, 155.1, 151.8, 148.9, 143.9, 139.7, 134.9, $130.5,129.4,128.9,128.9,128.2,128.1,128.0,126.8,118.8,116.4,39.1,39.0,32.2$, 31.1, 27.0, 26.9, 21.6. IR (ATR): $v_{\max }\left(\mathrm{cm}^{-1}\right)=3222(\mathrm{w}), 2973(\mathrm{~m}), 2931(\mathrm{w}), 2873(\mathrm{w})$, $2356(\mathrm{w}), 1754(\mathrm{~s}), 1606(\mathrm{~m}), 1496(\mathrm{~m}), 1479(\mathrm{~m}), 1397(\mathrm{~m}), 1343(\mathrm{~m}), 1279(\mathrm{w}), 1169$ (s), $1096(\mathrm{~s}), 1029(\mathrm{~m}), 903(\mathrm{~m}), 813(\mathrm{~m}), 755(\mathrm{w}), 703(\mathrm{~m}), 667(\mathrm{~s}), 575(\mathrm{~m})$. HRMS (ESI $\left.{ }^{+}\right)$ Calcd. for $\mathrm{C}_{32} \mathrm{H}_{38} \mathrm{~N}_{2} \mathrm{O}_{6} \mathrm{Na}$. [M+Na] ${ }^{+}: 601.2348$. Found: 601.2340. TLC (EtOAc-hexanes $=$ 50:50): $R_{\mathrm{f}}=0.38$ 


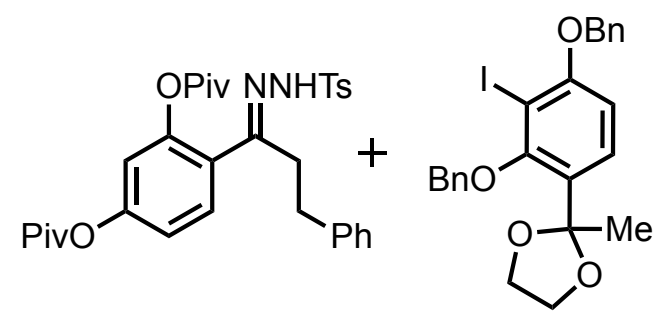

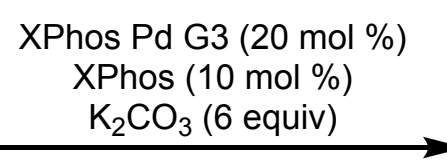

$\mathrm{PhF}, 85^{\circ} \mathrm{C}, 20 \mathrm{~h}$ (89\%)<smiles>CCCOc1ccc(C(=CCc2ccccc2)c2c(OCc3ccccc3)ccc(C3(C)OCCO3)c2OCc2ccccc2)c(OCc2ccccc2)c1</smiles>

\section{Alkene SI-4}

A dry $1 \mathrm{~L}$ round-bottomed flask was charged with 7 (5.02 g, $10.0 \mathrm{mmol}, 1$ equiv), XPhos Pd G3 (1.7 g, 2 mmol, $20 \mathrm{~mol} \%$ ), XPhos (475 mg, $1 \mathrm{mmol}, 10 \mathrm{~mol} \%$ ), and $\mathrm{K}_{2} \mathrm{CO}_{3}$ $(6.9 \mathrm{~g}, 50 \mathrm{mmol}, 5$ equiv), followed by dry $\mathrm{PhF}(150 \mathrm{~mL})$. The flask was sealed and resulting suspension sparged with argon for 15 minutes before being left under a static atmosphere of argon. The flask was then warmed to $80^{\circ} \mathrm{C}$. To a separate, dry $250 \mathrm{~mL}$ Erlenmeyer flask equipped with a 24/40 ground-glass joint was added SI-3 (11.5 g, 20.0 $\mathrm{mmol}, 2.0$ equiv). The flask was then sealed and purged with an atmosphere of argon before PhF $(150 \mathrm{~mL})$ was added via syringe. The resulting suspension was swirled until complete dissolution (ca. 15 minutes). The solution of SI-3 was then added slowly (ca 20 $\mathrm{mL} / \mathrm{h}$ ) to the suspension containing 7 at $80{ }^{\circ} \mathrm{C}$. After the addition was complete, the reaction let to stir for 8 hours before being cooled to $24^{\circ} \mathrm{C}$, filtered over a pad of silica gel. The silica gel pad was washed with EtOAc $(5 \times 150 \mathrm{~mL})$ and the washes combined with the filtrate before being concentrated in vacuo to give a sticky red oil. The oil was chromatographed (MPLC, $220 \mathrm{~g}$ column, $120 \mathrm{~mL} / \mathrm{min}$ hexanes $\rightarrow 40 \%$ EtOAc/hexanes to give $\mathbf{S I}-4$ as an orange foam $(6.68 \mathrm{~g}, 89 \%)$ (Note 1).

${ }^{1} \mathrm{H}$ NMR: $\left(600 \mathrm{MHz}, \mathrm{CDCl}_{3}\right): \delta 7.34-7.29(\mathrm{~m}, 2 \mathrm{H}), 7.26-7.21(\mathrm{~m}, 8 \mathrm{H}), 7.08-7.03(\mathrm{~m}$, $5 \mathrm{H}), 6.99-6.92(\mathrm{~m}, 3 \mathrm{H}), 6.86(\mathrm{dd}, \mathrm{J}=8.5,2.4 \mathrm{~Hz}, 1 \mathrm{H}), 6.53(\mathrm{~d}, \mathrm{~J}=8.7 \mathrm{~Hz}, 1 \mathrm{H}), 6.43(\mathrm{t}$, $\mathrm{J}=7.2 \mathrm{~Hz}, 1 \mathrm{H}), 5.03(\mathrm{~s}, 2 \mathrm{H}), 4.90(\mathrm{~s}, 2 \mathrm{H}), 4.02-3.98(\mathrm{~m}, 2 \mathrm{H}), 3.85-3.82(\mathrm{~m}, 2 \mathrm{H})$, $3.54(\mathrm{~d}, \mathrm{~J}=7.2 \mathrm{~Hz}, 2 \mathrm{H}), 1.78(\mathrm{~s}, 3 \mathrm{H}), 1.39$ (s, $9 \mathrm{H}), 1.22$ (s, $9 \mathrm{H}) .{ }^{13} \mathrm{C}$ NMR (151 MHz, $\left.\mathrm{CDCl}_{3}\right): \delta 176.6,176.4,157.1,155.4,149.7,148.9,140.1,138.1,137.1,135.2,130.2$, 130.1, 128.7, 128.4, 128.3, 128.3, 128.2, 128.0, 127.9, 127.8, 127.5, 127.5, 127.3, 127.1, 127.1, 127.0, 126.9, 126.2, 125.7, 118.0, 116.3, 108.8, 107.4, 74.7, 69.3, 64.3, 39.1, 39.0, 36.3. 27.1, 27.0, 26.7, 26.3. IR (ATR): $v_{\max }\left(\mathrm{cm}^{-1}\right)=2974(\mathrm{~m}), 2933(\mathrm{w}), 2871(\mathrm{~m}), 1752$ (s), $1604(\mathrm{w}), 1583(\mathrm{~m}), 1497(\mathrm{w}), 1454(\mathrm{~m}), 1399(\mathrm{~m}), 1260$ (w), 1107 (vs), 1071 (s), 1042 (s), 1029 (s), 901 (m), 732 (s), 697 (s), 561 (m). HRMS (ESI+) Calcd. for $\mathrm{C}_{49} \mathrm{H}_{52} \mathrm{O}{ }_{8} \mathrm{Na} .[\mathrm{M}+\mathrm{Na}]^{+}:$791.3560. Found: 791.3555. TLC $($EtOAc-hexanes $=25: 75): R_{\mathrm{f}}=$ 0.60

Note 1: If the reaction stalls, as judged by removing an aliquot and analyzing by ${ }^{1} \mathrm{H}$ NMR, another portion of $\mathrm{K}_{2} \mathrm{CO}_{3}$ typically restarts forward progress. 


\section{Table S1. Optimization Trials for the coupling of SI-3 and 7 to SI-4.}

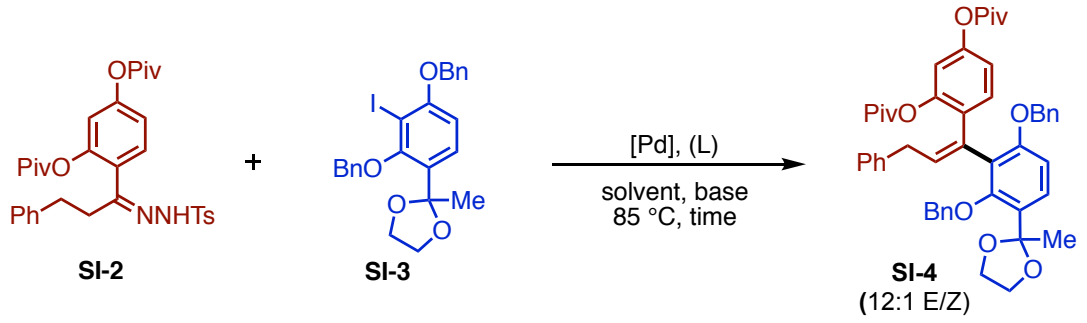

\begin{tabular}{|c|c|c|c|c|c|c|c|c|}
\hline entry & $\begin{array}{l}\text { Equiv } 9 \\
\text { (addition time) }\end{array}$ & [Pd] & (L) & $\begin{array}{l}\text { [Pd] loading ( } \mathrm{mol} \%) \\
\text { (L) loading ( } \mathrm{mol} \%)\end{array}$ & Base (equiv) & solvent & time $(h)^{1}$ & Yield $(\%)^{2}$ \\
\hline 1 & $1.2(\mathrm{n} / \mathrm{a})$ & $\mathrm{Pd}(\mathrm{MeCN})_{2} \mathrm{Cl}_{2}$ & XPhos & 5,10 & $\mathrm{NaO}^{t} \mathrm{Bu}(3.0)$ & $\mathrm{PhF}$ & 3 & 7 \\
\hline 1 & $1.2(\mathrm{n} / \mathrm{a})$ & $\mathrm{Pd}(\mathrm{MeCN})_{2} \mathrm{Cl}_{2}$ & XPhos & 10,20 & $\mathrm{NaO}^{\mathrm{t}} \mathrm{Bu}(3.0)$ & $\mathrm{PhF}$ & 4 & 8 \\
\hline 2 & $1.2(1.75 \mathrm{~h})$ & $\mathrm{Pd}(\mathrm{MeCN})_{2} \mathrm{Cl}_{2}$ & XPhos & 10,20 & $\mathrm{~K}_{2} \mathrm{CO}_{3}(3.0)$ & $\mathrm{PhF}$ & 2 & 26 \\
\hline 3 & $3.0(8 \mathrm{~h})$ & $\mathrm{Pd}(\mathrm{MeCN})_{2} \mathrm{Cl}_{2}$ & XPhos & 10,20 & $\mathrm{~K}_{2} \mathrm{CO}_{3}(3.1)$ & $\mathrm{PhF}$ & 1 & 37 \\
\hline 4 & $4.3(8 \mathrm{~h})$ & $\mathrm{Pd}(\mathrm{MeCN})_{2} \mathrm{Cl}_{2}$ & XPhos & 10,20 & $\mathrm{~K}_{2} \mathrm{CO}_{3}(4.5)$ & $\mathrm{PhF}$ & 1 & 53 \\
\hline 5 & $4.5(8 \mathrm{~h})$ & $\mathrm{Pd}(\mathrm{MeCN})_{2} \mathrm{Cl}_{2}$ & XPhos & 10,20 & $\mathrm{~K}_{2} \mathrm{CO}_{3}(4.5)$ & 1,4-dioxane & 1 & 43 \\
\hline 6 & $4.5(12 \mathrm{~h})$ & $\mathrm{Pd}(\mathrm{MeCN})_{2} \mathrm{Cl}_{2}$ & XPhos & 20,40 & $\mathrm{~K}_{2} \mathrm{CO}_{3}(6.0)$ & $\mathrm{PhF}$ & 12 & $96-99^{3}$ \\
\hline 7 & $4.0(12 \mathrm{~h})$ & XPhos Pd G3 & XPhos & 20,20 & $\mathrm{~K}_{2} \mathrm{CO}_{3}(6.0)$ & PhF/dioxane (1:1) & 12 & 96 \\
\hline 8 & $2.5(12 \mathrm{~h})$ & XPhos Pd G3 & XPhos & 20,20 & $\mathrm{~K}_{2} \mathrm{CO}_{3}(6.0)$ & $\mathrm{PhF}$ & 12 & $87-94^{4}$ \\
\hline 9 & $2.0(8 \mathrm{~h})$ & XPhos Pd G3 & XPhos & 20,10 & $\mathrm{~K}_{2} \mathrm{CO}_{3}(6.0)$ & $\mathrm{PhF}$ & 14 & $83-89^{5}$ \\
\hline 10 & $2.0(8 \mathrm{~h})$ & XPhos Pd G3 & XPhos & 20,20 & $\mathrm{~K}_{2} \mathrm{CO}_{3}(6.0)$ & PhMe & 12 & $<5$ \\
\hline 11 & $2.0(8 \mathrm{~h})$ & XPhos Pd G3 & XPhos & 20,20 & $\mathrm{~K}_{2} \mathrm{CO}_{3}(6.0)$ & DMA & 12 & $<5$ \\
\hline 12 & $3: 1(8 \mathrm{~h})$ & XPhos Pd G2 & XPhos & 20,20 & $\mathrm{~K}_{2} \mathrm{CO}_{3}(6.0)$ & $\mathrm{PhF}$ & 12 & $<5$ \\
\hline 13 & $3: 1(8 \mathrm{~h})$ & ${ }^{t}$ BuXPhos Pd G3 & ${ }^{t}$ BuXPhos & 20,20 & $\mathrm{~K}_{2} \mathrm{CO}_{3}(6.0)$ & $\mathrm{PhF}$ & 12 & $<5$ \\
\hline & $\begin{array}{l}\text { Other li } \\
\text { SPhos, RuP }\end{array}$ & $\begin{array}{l}\text { igands which led } \\
\text { Phos, JohnPhos, De }\end{array}$ & $\begin{array}{l}\text { no conver } \\
\text { ePhos, Bre }\end{array}$ & $\begin{array}{l}\text { ion to SI-4: } \\
\text { Phos, Xantphos }\end{array}$ & \multicolumn{3}{|c|}{$\begin{array}{l}\text { Bases which led to poorconversion to SI-4: } \\
\qquad \mathrm{Cs}_{2} \mathrm{CO}_{3}, \mathrm{LiO}^{t} \mathrm{Bu}, \mathrm{CsF}, \mathrm{K}_{2} \mathrm{HPO}_{4}\end{array}$} & \\
\hline
\end{tabular}

${ }^{1}$ Reflects time after addition of SI-4 was complete. ${ }^{2}$ Isolated yield. Reaction scale (10): ${ }^{3} 100 \mathrm{mg}-1 \mathrm{~g} .{ }^{4} 1 \mathrm{~g}-5 \mathrm{~g} .{ }^{5} 4 \mathrm{~g}-8 \mathrm{~g}$. 

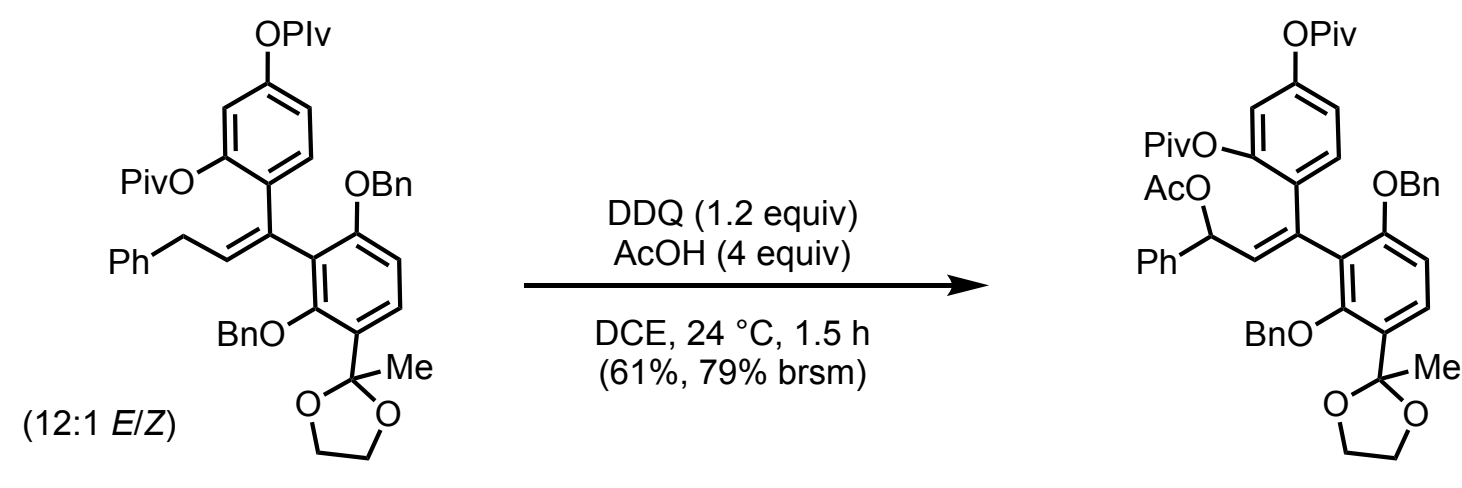

\section{Acetate SI-5}

To a solution of SI-4 $(5.85 \mathrm{~g}, 7.61 \mathrm{mmol})$ in dry DCE $(55 \mathrm{~mL})$ was added $\mathrm{AcOH}$ (1.91 mL, $30.4 \mathrm{mmol}, 4$ equiv) and activated 4 A molecular sieves $(2.8 \mathrm{~g})$. The suspension was stirred for 2 minutes before freshly recrystallized DDQ (1.89 g, $8.37 \mathrm{mmol}, 1.1$ equiv) was added in a single portion. The suspension was then allowed to stir for 1.5 hours at $24 \mathrm{C}$ before being quenched with saturated aqueous $\mathrm{NaHCO}_{3}(100 \mathrm{~mL})$. The biphasic mixture was then filtered through filter paper and the biphasic solution washed with EtOAc $(3 \times 100 \mathrm{~mL})$. The combined organic phases were washed with saturated aqueous $\mathrm{NaHCO}_{3}(100 \mathrm{~mL})$, brine $(100 \mathrm{~mL})$, dried over $\mathrm{Na}_{2} \mathrm{SO}_{4}$, filtered and concentrated to yield an orange oil. The resulting oil was chromatographed (MPLC, $120 \mathrm{~g}$ column, $80 \mathrm{~mL} / \mathrm{min}$, $10 \%$ hexanes $\rightarrow 40 \%$ EtOAc/hexanes to give SI-5 as a colorless foam (3.93 $\mathrm{g}, 61 \%, 79 \%$ BRSM, Note 1).

Note 1: An early fraction contained SI-5 $(915 \mathrm{mg})$ as a 1:1.2 mixture of $E / Z$ isomers.

1H NMR: ${ }^{1} \mathrm{H}$ NMR $\left(600 \mathrm{MHz}, \mathrm{CDCl}_{3}\right): \delta 7.33(\mathrm{~d}, J=8.8 \mathrm{~Hz}, 1 \mathrm{H}), 7.29-7.19(\mathrm{~m}, 8 \mathrm{H})$, $7.16-7.12(\mathrm{~m}, 3 \mathrm{H}), 7.12-7.08(\mathrm{~m}, 2 \mathrm{H}), 6.98-6.94(\mathrm{~m}, 2 \mathrm{H}), 6.92(\mathrm{~d}, J=2.3 \mathrm{~Hz}, 1 \mathrm{H})$, $6.76(\mathrm{dd}, J=8.5,2.3 \mathrm{~Hz}, 1 \mathrm{H}), 6.58-6.57(\mathrm{~m}, 1 \mathrm{H}), 6.54(\mathrm{~d}, J=8.8 \mathrm{~Hz}, 1 \mathrm{H}), 4.96-4.83$ $(\mathrm{m}, 4 \mathrm{H}), 4.00-3.91(\mathrm{~m}, 2 \mathrm{H}), 3.84-3.75(\mathrm{~m}, 2 \mathrm{H}), 1.75(\mathrm{~s}, 3 \mathrm{H}), 1.73(\mathrm{~s}, 3 \mathrm{H}), 1.36(\mathrm{~s}, 9 \mathrm{H})$, 1.15 (s, 9H). $\left.{ }^{13} \mathrm{C} \mathrm{NMR} \mathrm{(151} \mathrm{MHz,} \mathrm{CDCl}_{3}\right): \delta$ 176.40, 176.30, 169.71, 157.03, 155.46, $150.15,148.49,139.84,138.34,137.01$, 133.90, 130.75, 130.30, 129.55, 129.34, 128.91, $128.40,128.30,127.93,127.83,127.44,127.21,127.15,127.14,126.85,126.75,125.94$, 118.01, 116.53, 108.73, 107.66, 77.23, 74.50, 73.96, 69.48, 64.35, 64.34, 39.13, 38.96, 27.18, 26.97, 26.32, 20.87. IR (ATR): $v_{\max }\left(\mathrm{cm}^{-1}\right)=2973(\mathrm{~m}), 2933(\mathrm{~m}), 2674(\mathrm{w}), 2255$ (w), 1752 (s), 1745 (s), 1605 (w), 1585 (m), 1369 (m), 1230 (s), 1099 (vs), 1071 (s), 1043 (m), $868(\mathrm{~m}), 697$ (s), 665 (s). HRMS (ESI+) Calcd. for $\mathrm{C}_{51} \mathrm{H}_{54} \mathrm{O}_{10} \mathrm{Na}$. [M+Na] ${ }^{+}: 849.3615$. Found: 849.3610. TLC (EtOAc-hexanes $=25: 75): R_{\mathrm{f}}=0.43$ 
<smiles>[C+]OC(C=C(c1ccc(OCCCC)cc1OCc1ccccc1)c1c(OCc2ccccc2)ccc(C2(C)OCCO2)c1OCc1ccccc1)c1ccccc1</smiles>

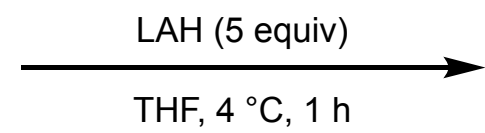

$(92 \%)$<smiles>CC(C)(C)Oc1ccc(C2(C)OCCO2)c(C(=CC(O)c2ccccc2)c2ccc(O)cc2O)c1OCc1ccccc1</smiles>

\section{Triol SI-6}

A dry $500 \mathrm{~mL}$ three-necked, round-bottom flask equipped with pressureequalizing addition funnel was charged with $\mathrm{LiAlH}_{4}(0.642 \mathrm{~g}, 16.9 \mathrm{mmol}, 5$ equiv). The reaction flask purged with an atmosphere of nitrogen and cooled to $4{ }^{\circ} \mathrm{C}$ and THF (100 $\mathrm{mL}$ ) was added. To this suspension was added dropwise a solution of SI-5 (3.48g, 4.23 $\mathrm{mmol})$ in THF (100 mL) through the addition funnel over the course of 30 minutes. The addition funnel was rinsed with additional THF $(15 \mathrm{~mL})$ to ensure full transfer of SI-5. After the addition was complete, the reaction allowed to stir at $4{ }^{\circ} \mathrm{C}$ for 1 hour before being carefully quenched by dropwise addition of saturated aqueous ammonium chloride (100 $\mathrm{mL})$. To the biphasic suspension was added EtOAc $(100 \mathrm{~mL})$ and the contents transferred to a $1 \mathrm{~L}$ separatory funnel. The aqueous phase was separated and washed with EtOAc $(3 \times 150 \mathrm{~mL})$ and the combined organics washed with saturated sodium sulfate solution $(100 \mathrm{~mL})$, dried over sodium sulfate, filtered, and concentrated to give a colorless oil. The resulting oil was chromatographed (MPLC, $120 \mathrm{~g}$ column, $80 \mathrm{~mL} / \mathrm{min}, 30 \%$ EtOAc/hexanes $\rightarrow 90 \%$ EtOAc/hexanes to give SI-6 as a colorless microcrystalline solid (2.41 g, 92\%).

1H NMR: (400 MHz, DMSO-d6): $9.19(\mathrm{~s}, 1 \mathrm{H}), 8.39(\mathrm{~s}, 1 \mathrm{H}), 7.34-7.29(\mathrm{~m}, 12 \mathrm{H}), 7.19-$ $7.15(\mathrm{~m}, 3 \mathrm{H}), 6.96(\mathrm{~d}, J=8.4 \mathrm{~Hz}, 1 \mathrm{H}), 6.87(\mathrm{~d}, J=8.8 \mathrm{~Hz}, 1 \mathrm{H}), 6.18(\mathrm{~d}, J=2.4 \mathrm{~Hz}, 1 \mathrm{H})$, $6.06(\mathrm{dd}, J=8.4,2.4 \mathrm{~Hz}, 1 \mathrm{H}), 5.96(\mathrm{~d}, J=9.4 \mathrm{~Hz}, 1 \mathrm{H}), 5.45(\mathrm{~d}, J=5.6 \mathrm{~Hz}, 1 \mathrm{H}), 5.15$ (dd, $J=9.9,4.4 \mathrm{~Hz}, 1 \mathrm{H}), 5.05(\mathrm{~s}, 2 \mathrm{H}), 4.90(\mathrm{~s}, 1 \mathrm{H}), 4.05-3.91(\mathrm{~m}, 2 \mathrm{H}), 3.89-3.68(\mathrm{~m}, 2 \mathrm{H})$, 1.59 (s, 3H). ${ }^{13} \mathrm{C}$ NMR (151 MHz, $\left.\mathrm{CDCl}_{3}\right): \delta$ 158.11, 156.96, 155.47, 154.14, 145.17, 138.32, 138.05, 137.34, 132.27, 130.27, 128.99, 128.97, 128.73, 128.45, 128.09, 128.01, $127.14,126.69,126.42,117.23,108.43,108.20,106.54,102.85,76.23,70.21$ (overlap), $64.43,64.42,26.70 .{ }^{*} 9$ aromatic carbons and 1 aliphatic carbon (70.2) are overlapping. IR (ATR): $v_{\max }\left(\mathrm{cm}^{-1}\right)=3273(\mathrm{br}), 3062(\mathrm{~m}), 3032(\mathrm{~m}), 2964(\mathrm{~m}), 2887(\mathrm{~m}), 2254(\mathrm{w})$, $1618(\mathrm{~m}), 1560(\mathrm{~m}), 1506(\mathrm{~m}), 1454(\mathrm{~m}), 1418(\mathrm{~m}), 1371(\mathrm{~m}), 1313(\mathrm{w}), 1249(\mathrm{~m}), 1191$ (s), 1066 (s), 1025 (s), 1003 (s), 912 (w), 848 (m), 738 (s), 699 (s), 550 (m). HRMS (ESI+) Calcd. for $\mathrm{C}_{39} \mathrm{H}_{36} \mathrm{O}_{7} \mathrm{Na}$. [M+Na]+: 639.2359, Found: 639.2355 TLC (EtOAc-hexanes = 50:50): $R_{\mathrm{f}}=0.28$ 
<smiles>CC1(c2ccc(CCc3ccccc3)c(C(=CC(O)c3ccccc3)c3ccc(O)cc3O)c2OCc2ccccc2)OCCO1</smiles>

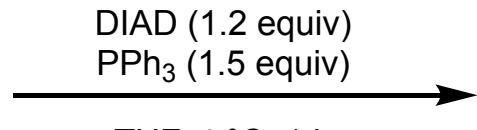

THF, $4{ }^{\circ} \mathrm{C}, 1 \mathrm{~h}$

$(81 \%, 1: 1.1 \mathrm{dr})$

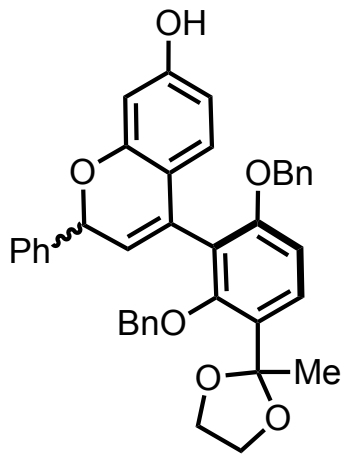

(1:1.1 mixture of atropisomers)

\section{Phenol 15}

A dry $500 \mathrm{~mL}$ Schlenk flask was charged with SI-6 (2.35 g, $3.81 \mathrm{mmol}, 1$ equiv) and THF $(120 \mathrm{~mL})$. The flask was then sealed and sparged with dry argon for 15 minutes before being left under a static atmosphere of argon and stirred at $4{ }^{\circ} \mathrm{C}$. Triphenylphosphine (1.48 g, $5.78 \mathrm{mmol}, 1.5$ equiv) was then added in one portion. After 5 minutes, DIAD ( $0.82 \mathrm{ml}, 4.57 \mathrm{mmol}, 1.2$ equiv) was added via syringe and the resulting dark solution stirred for $1 \mathrm{~h}$ at $4{ }^{\circ} \mathrm{C}$. After $1 \mathrm{~h}$, degassed silica (ca. $20 \mathrm{~g}$, Note 1) was added in one portion and the reaction evacuated under crude vacuum (ca. 15 torr) while warming to room temperature under the aid of a water bath. The dried material was chromatographed directly via MPLC (120 g column, $80 \mathrm{~mL} / \mathrm{min}, 100 \% \mathrm{DCM}$ ) to give 15 as a colorless foam $(1.86 \mathrm{~g}, 81 \%)$ and as a $1: 1.1$ mixture of inseparable atropisomers (Note 2)

Note 1: Silica was degassed by evacuating for 2 minutes under vacuum (ca. 15 torr).

Note 2: 15 is particularly sensitive to oxygen and thus should be stored under argon at $20{ }^{\circ} \mathrm{C}$. Significant coloration (pink-burgundy) can be observed under these conditions over the course of 4 days, but the overall purity doesn't appear to degrade significantly. Significant degradation can be observed on storage over the course of several weeks.

${ }^{1} \mathrm{H}$ NMR $\left(600 \mathrm{MHz}, \mathrm{CDCl}_{3}\right.$, both atropisomers): $\delta 7.56-7.48(\mathrm{~m}, 2 \mathrm{H}), 7.44-7.37(\mathrm{~m}$, 4H), $7.36-7.27(\mathrm{~m}, 6 \mathrm{H}), 7.27-7.24(\mathrm{~m}, 9 \mathrm{H}), 7.24-7.14(\mathrm{~m}, 8 \mathrm{H}), 7.15-7.06(\mathrm{~m}, 4$ H), $6.81-6.75(\mathrm{~m}, 3 \mathrm{H}), 6.73(\mathrm{~d}, J=8.3 \mathrm{~Hz}, 1 \mathrm{H}), 6.44(\mathrm{~d}, J=2.5 \mathrm{~Hz}, 1 \mathrm{H}), 6.35(\mathrm{~d}, J=$ $2.5 \mathrm{~Hz}, 1 \mathrm{H}), 6.31-6.26(\mathrm{~m}, 2 \mathrm{H}), 6.00(\mathrm{~d}, J=3.5 \mathrm{~Hz}, 1 \mathrm{H}), 5.74(\mathrm{~d}, J=3.8 \mathrm{~Hz}, 1 \mathrm{H}), 5.73$ $(\mathrm{d}, J=3.5 \mathrm{~Hz}, 1 \mathrm{H}), 5.69(\mathrm{~d}, J=3.8 \mathrm{~Hz}, 1 \mathrm{H}), 5.10-5.02(\mathrm{~m}, 4 \mathrm{H}), 4.94(\mathrm{~d}, J=9.9 \mathrm{~Hz}, 1 \mathrm{H})$, $4.87-4.81(\mathrm{~m}, 2 \mathrm{H}), 4.77(\mathrm{~d}, J=7.2 \mathrm{~Hz}, 2 \mathrm{H}), 4.72(\mathrm{~d}, J=9.9 \mathrm{~Hz}, 1 \mathrm{H}), 4.12-4.02(\mathrm{~m}$, 2H), $4.00-3.94(\mathrm{~m}, 1 \mathrm{H}), 3.94-3.83(\mathrm{~m}, 3 \mathrm{H}), 1.81(\mathrm{~s}, 3 \mathrm{H}), 1.80(\mathrm{~s}, 3 \mathrm{H}) .{ }^{13} \mathrm{C}$ NMR (151 $\mathrm{MHz}, \mathrm{CDCl}_{3}$, both atropisomers): $\delta$ 157.6, 156.6, 156.6, 156.1, 155.9, 155.1, 154.5, $141.0,140.2$, 138.1, 138.1, 137.2, 136.9, 129.4, 128.8, 128.7, 128.6, 128.5, 128.5, 128.5, 128.4, 128.3, 128.2, 128.1, 127.8, 127.8, 127.8, 127.7, 127.7, 127.3, 127.2, 127.1, 127.1, 127.0, 126.8, 126.7, 126.5, 123.1, 123.0, 122.9, 122.7, 116.6, 116.5, 109.0, 108.9, 108.2, 
108.0, 107.6, 107.0, 103.7, 103.4, 77.6, 77.5, 76.0, 75.6, 70.4, 70.4, 64.6, 64.6, 64.5, 26.4, 26.4. IR (ATR): $v_{\max }\left(\mathrm{cm}^{-1}\right)=3340(\mathrm{br}), 3031(\mathrm{~m}), 2975(\mathrm{~m}), 2339(\mathrm{w}), 1580(\mathrm{~s})$, 1478 (s), 1453 (s), 1371 (s), 1263 (s), 1198 (s), 1089 (s), 1071 (s), 1042 (m), 952 (m), 735 (s), 696 (s). HRMS (ESI+) Calcd. for $\mathrm{C}_{39} \mathrm{H}_{34} \mathrm{O}_{6} \mathrm{Na}$. [M+Na] ${ }^{+}$: 621.2253. Found: 621.2244. TLC (DCM): $R_{\mathrm{f}}=0.40$. 

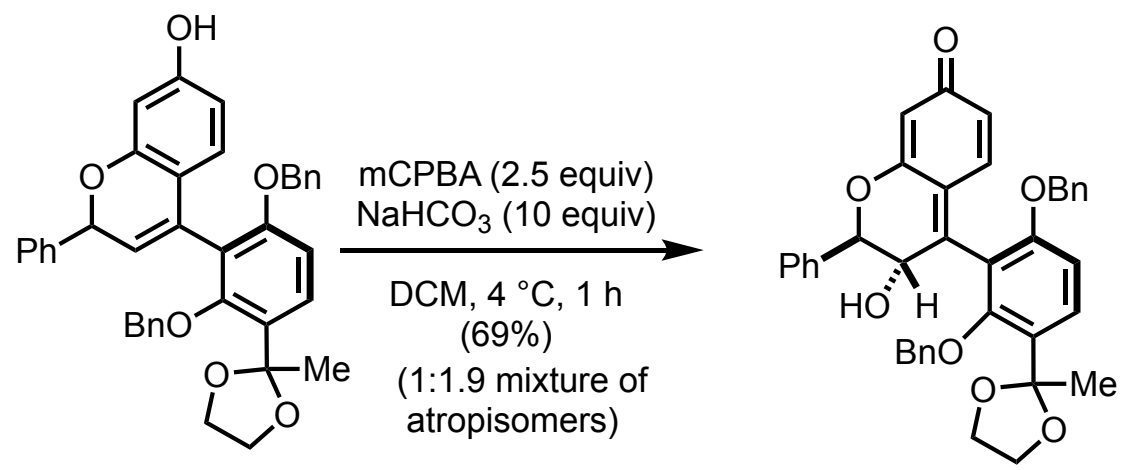

\section{p-quinone methide 14}

To a $100 \mathrm{~mL}$ round bottom flask equipped with magnetic stir-bar was added 15 (548 mg, $0.916 \mathrm{mmol})$ and DCM $(13 \mathrm{~mL})$. The stirred solution was cooled to $4{ }^{\circ} \mathrm{C}$ and a solid mixture containing mCPBA ( $514 \mathrm{mg}, 77 \%$ purity, $2.30 \mathrm{mmol}$ ) and $\mathrm{NaHCO}_{3}(761 \mathrm{mg}$, $9.05 \mathrm{mmol}$ ) were added in one portion. The suspension vigorously stirred at $4{ }^{\circ} \mathrm{C}$ for 45 minutes before being diluted with DCM $(25 \mathrm{~mL})$ and saturated brine $(50 \mathrm{~mL})$. The contents were then transferred to a separatory funnel and layers separated. The aqueous phase washed with DCM $(5 \times 25 \mathrm{~mL})$ and combined organic phases washed with saturated brine $\left(3 \times 50 \mathrm{~mL}\right.$ ) before being dried over $\mathrm{Na}_{2} \mathrm{SO}_{4}$, filtered. Silica gel (ca $10 \mathrm{~g}$ ) was added and the suspension concentrated in vacuo. The free-flowing solid then chromatographed directly (EtOAc-hexanes 1:3 --> 1:1) to give 14 as a yellow foam (385 mg, 69\% yield) and as a 1:1.9 mixture of atropisomers.

Note 1. The isomers could not be separated by silica gel column or MPLC but could be separated by semi-preparative HPLC (Ethyl acetate-hexanes 10:1 $\rightarrow$ 3:1). The two atropisomers were separated and characterized independently.

Note 2. We concluded that the mixture contained two atropisomers, and not cis/trans diastereomers of the C-ring hydroxyl and phenyl moieties by separating the two compounds by semi-prep HPLC and analyzing the relevant coupling constants. In particular, the 2"-3" $\mathrm{H}-\mathrm{H}$ coupling constants were virtually the same $(10.4$ and $10.6 \mathrm{~Hz}$ for the less polar and more polar diastereomers, respectively). A COSY spectrum of each atropisomer is included in the spectral section.

TLC $\left(\right.$ EtOAc-hexanes = 1:1): both atroisomers, $R_{\mathrm{f}}=0.30$

14 (less polar diastereomer): ${ }^{1} \mathrm{H}$ NMR (400 MHz, Chloroform- $d$ ): $\delta 7.60$ (d, $J=8.7 \mathrm{~Hz}$, $1 \mathrm{H}), 7.42-7.28(\mathrm{~m}, 12 \mathrm{H}), 7.24-7.18(\mathrm{~m}, 2 \mathrm{H}), 6.93(\mathrm{~d}, J=9.9 \mathrm{~Hz}, 1 \mathrm{H}), 6.87(\mathrm{~d}, J=8.8$ $\mathrm{Hz}, 1 \mathrm{H}), 6.26(\mathrm{~d}, J=9.4 \mathrm{~Hz}, 1 \mathrm{H}), 5.96(\mathrm{~d}, J=1.9 \mathrm{~Hz}, 1 \mathrm{H}), 5.08(\mathrm{~d}, J=10.4 \mathrm{~Hz}, 1 \mathrm{H}), 5.04$ (s, 2H) 10. $4.91-4.78(\mathrm{~m}, 2 \mathrm{H}), 4.49(\mathrm{~d}, J=10.4 \mathrm{~Hz}, 1 \mathrm{H}), 4.16-4.05(\mathrm{~m}, 2 \mathrm{H}), 4.01-$ $3.82(\mathrm{~m}, 1 \mathrm{H}), 1.77(\mathrm{~s}, 3 \mathrm{H})$.

14 (more polar diastereomer): ${ }^{1} \mathrm{H}$ NMR (400 MHz, Chloroform- $d$ ): $\delta 7.51$ (d, $J=8.9 \mathrm{~Hz}$, $1 \mathrm{H}), 7.34-7.21(\mathrm{~m}, 9 \mathrm{H}), 7.12(\mathrm{~d}, J=7.2 \mathrm{~Hz}, 2 \mathrm{H}), 7.01-6.91(\mathrm{~m}, 2 \mathrm{H}), 6.73(\mathrm{~d}, J=8.8$ 
$\mathrm{Hz}, 1 \mathrm{H}), 6.26(\mathrm{~s}, 1 \mathrm{H}), 5.97(\mathrm{~s}, 1 \mathrm{H}), 5.34(\mathrm{~d}, J=10.6 \mathrm{~Hz}, 1 \mathrm{H}), 5.00(\mathrm{~d}, J=3.5 \mathrm{~Hz}, 2 \mathrm{H})$, $4.45-4.34(\mathrm{~m}, 3 \mathrm{H}), 4.08-3.73(\mathrm{~m}, 3 \mathrm{H}), 1.77(\mathrm{~s}, 3 \mathrm{H})$.

14 (mixture): ${ }^{1} \mathrm{H}$ NMR (600 MHz, Chloroform- $d$ ): $\delta 7.63-7.55(\mathrm{~m}, 2 \mathrm{H}), 7.03(\mathrm{~d}, J=9.8$ $\mathrm{Hz}, 1 \mathrm{H}), 6.93(\mathrm{~d}, J=9.9 \mathrm{~Hz}, 1 \mathrm{H}), 6.87(\mathrm{~d}, J=8.8 \mathrm{~Hz}, 1 \mathrm{H}), 6.79(\mathrm{~d}, J=8.9 \mathrm{~Hz}, 1 \mathrm{H}), 6.34$ $(\mathrm{dd}, J=9.8,1.8 \mathrm{~Hz}, 1 \mathrm{H}), 6.27(\mathrm{dd}, J=9.9,1.9 \mathrm{~Hz}, 1 \mathrm{H}), 6.04(\mathrm{~d}, J=1.8 \mathrm{~Hz}, 1 \mathrm{H}), 5.96(\mathrm{dd}$, $J=6.2,1.8 \mathrm{~Hz}, 1 \mathrm{H}), 5.41(\mathrm{~d}, J=10.8 \mathrm{~Hz}, 1 \mathrm{H}), 5.18-4.99(\mathrm{~m}, 6 \mathrm{H}), 4.88-4.78(\mathrm{~m}, 2 \mathrm{H})$, $4.54-4.40(\mathrm{~m}, 3 \mathrm{H}), 4.17-4.04(\mathrm{~m}, 5 \mathrm{H}), 4.03-3.82(\mathrm{~m}, 5 \mathrm{H}), 1.85(\mathrm{~s}, 2 \mathrm{H}), 1.77(\mathrm{~s}, 4 \mathrm{H})$. ${ }^{13} \mathrm{C}$ NMR (151 MHz, $\left.\mathrm{CDCl}_{3}\right): \delta 188.3,188.2,161.2,161.0,156.7,156.3,156.0,155.5$, $150.7,145.7,137.6,137.0,136.9,136.1,136.0,135.74134 .2,133.9,133.8,130.0$, $129.94129 .5,129.2$, 129.1, 129.1, 129.0, 128.9, 128.7, 128.7, 128.7, 128.6, 128.5, 128.5, $128.4,128.4,128.4,128.3,128.3,128.3,128.2,128.2,128.1,128.0,127.7,127.7,127.4$, $127.4,127.2,127.2,127.0,126.9,126.6,126.4,125.2,121.0,118.6,109.0,108.9,108.8$, $108.4,108.3,108.2,108.1,83.0,82.6,80.4,77.5,77.5,71.0,70.7,70.1,69.0,64.7,64.6$, 64.5, 64.5, 26.5, 26.3. IR (ATR): $v_{\max }\left(\mathrm{cm}^{-1}\right)=3373(\mathrm{~s}), 2926(\mathrm{~m}), 1761(\mathrm{~s}), 1736(\mathrm{~m})$, $1617(\mathrm{~s}), 1593(\mathrm{~s}), 1496(\mathrm{~m}), 1370(\mathrm{~m}), 1284(\mathrm{~s}), 1212(\mathrm{~s}), 1110(\mathrm{~m}), 966(\mathrm{~m}), 805(\mathrm{~m})$, 753 (w). HRMS (ESI+) Calcd. for $\mathrm{C}_{39} \mathrm{H}_{34} \mathrm{NaO}_{7}$. [M+Na] ${ }^{+}:$637.2202. found: 637.2198. TLC $($ EtOAc-hexanes $=1: 1): R_{\mathrm{f}}=0.24$. 

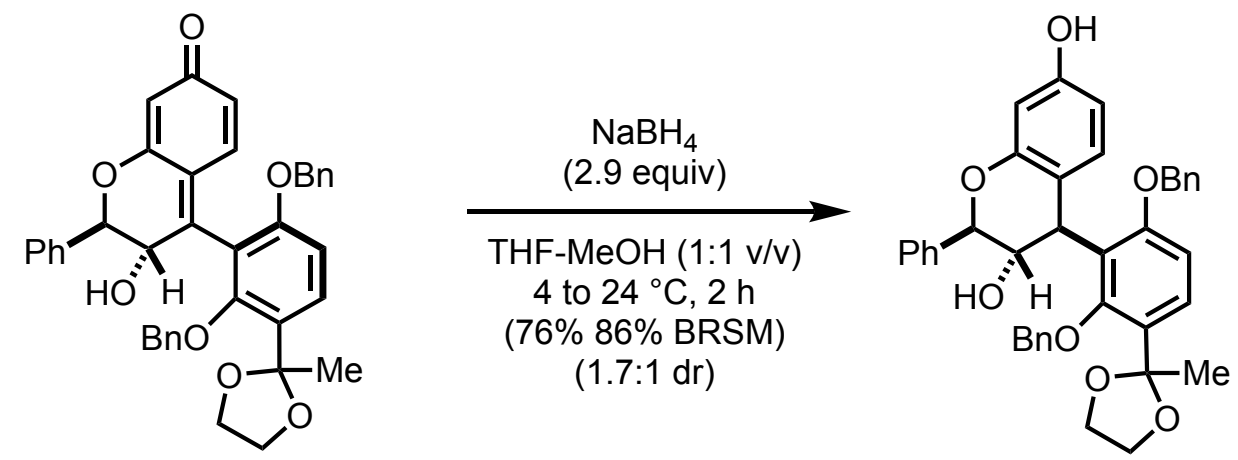

\section{Alcohol 16}

To a solution of $p$-quinone methide $14(0.298 \mathrm{mg}, 0.485 \mathrm{mmol})$ in a mixture of THF and $\mathrm{MeOH}(6 \mathrm{~mL}, 1: 1 \mathrm{v} / \mathrm{v})$ at $4{ }^{\circ} \mathrm{C}$ was added portion wise $\mathrm{NaBH}_{4}$ (3 portions over 45 mins, $48.6 \mathrm{mg}, 1.28 \mathrm{mmol})$. After the reaction was complete by TLC, EtOAc (10 mL) and saturated ammonium chloride $(10 \mathrm{~mL})$ was added and the contents transferred to a separatory funnel. The phases were separated, and the aqueous phase washed with EtOAc $(5 \times 10 \mathrm{~mL})$. The combined organics were washed with brine $(3 \times 10 \mathrm{~mL})$, dried over $\mathrm{Na}_{2} \mathrm{SO}_{4}$, and filtered. Silica gel (ca. $5 \mathrm{~g}$ ) was added and the solvents removed in vacuo. The free flowing solid was then chromatographed directly (EtOAc-hexanes 1:5 $\rightarrow$ $1: 1)$ to give 16 as a 1.7: mixture of diastereomers (228.3 $\mathrm{mg}, 76 \%)$. The major diastereomer is illustrated and the minor diastereomer was not assigned.

${ }^{1}$ H NMR (600 MHz, Chloroform- $d$ ): $\delta 7.63-7.57(\mathrm{~m}, 4 \mathrm{H}), 7.52-7.47(\mathrm{~m}, 3 \mathrm{H}), 7.43-$ $7.38(\mathrm{~m}, 4 \mathrm{H}), 7.38-7.28(\mathrm{~m}, 16 \mathrm{H}), 7.26-7.21(\mathrm{~m}, 14 \mathrm{H}), 7.20-7.15(\mathrm{~m}, 2 \mathrm{H}), 7.02-6.95$ $(\mathrm{m}, 4 \mathrm{H}), 6.85(\mathrm{~s}, 2 \mathrm{H}), 6.76-6.70(\mathrm{~m}, 3 \mathrm{H}), 6.59(\mathrm{~d}, J=8.4 \mathrm{~Hz}, 1 \mathrm{H}), 6.56(\mathrm{dd}, J=8.4,1.1$ $\mathrm{Hz}, 2 \mathrm{H}), 6.45(\mathrm{~d}, J=2.5 \mathrm{~Hz}, 1 \mathrm{H}), 6.35(\mathrm{~d}, J=2.5 \mathrm{~Hz}, 2 \mathrm{H}), 6.33(\mathrm{dd}, J=8.4,2.6 \mathrm{~Hz}, 1 \mathrm{H})$, 6.29 (dd, $J=8.4,2.6 \mathrm{~Hz}, 2 \mathrm{H}), 5.47(\mathrm{~d}, J=4.1 \mathrm{~Hz}, 1 \mathrm{H}), 5.35(\mathrm{~d}, J=10.2 \mathrm{~Hz}, 2 \mathrm{H}), 5.11(\mathrm{~d}$, $J=10.3 \mathrm{~Hz}, 2 \mathrm{H}), 4.92-4.84(\mathrm{~m}, 4 \mathrm{H}), 4.84-4.75(\mathrm{~m}, 6 \mathrm{H}), 4.75-4.69(\mathrm{~m}, 5 \mathrm{H}), 4.63(\mathrm{td}$, $J=9.4,3.1 \mathrm{~Hz}, 2 \mathrm{H}), 4.53(\mathrm{~d}, J=7.5 \mathrm{~Hz}, 1 \mathrm{H}), 4.17-4.06(\mathrm{~m}, 6 \mathrm{H}), 4.03-3.90(\mathrm{~m}, 5 \mathrm{H})$, $1.89(\mathrm{~s}, 5 \mathrm{H}), 1.79(\mathrm{~s}, 3 \mathrm{H}) .{ }^{13} \mathrm{C}$ NMR (151 MHz, $\left.\mathbf{C D C l}_{3}\right): \delta$ 157.7, 157.7, 155.1, 155.0, 154.9, 154.6, 138.3, 138.1, 137.7, 136.4, 128.9, 128.8, 128.6, 128.6, 128.6, 128.5, 128.4, 128.3, 128.3, 128.2, 127.9, 127.8, 127.8, 127.7, 127.4, 127.3, 127.2, 126.9, 126.7, 124.5, 124.1, 118.1, 108.9, 108.4, 108.3, 108.1, 107.7, 103.0, 102.9, 82.8, 78.5, 70.2, 70.0, 69.8, 69.7, 64.4, 64.4, 64.4, 41.3, 37.9, 26.4, 26.2. IR (ATR): $v_{\max }\left(\mathrm{cm}^{-1}\right)=3307(\mathrm{~m}), 3246(\mathrm{~m})$, $2924(\mathrm{~m}), 2888(\mathrm{~m}), 1622(\mathrm{~s}), 1510(\mathrm{~m}), 1455(\mathrm{~s}), 1388(\mathrm{~m}), 1208$ (vs), 1170 (vs), 1081 (vs), 1000 (m), 847 (s), 695 (s). HRMS (ESI+) Calcd. for $\mathrm{C}_{39} \mathrm{H}_{36} \mathrm{NaO}_{7}$. [M+Na]+: 639.2359. found: 639.2348 TLC (EtOAc-hexanes $=1: 1): R_{f}=0.48$. 


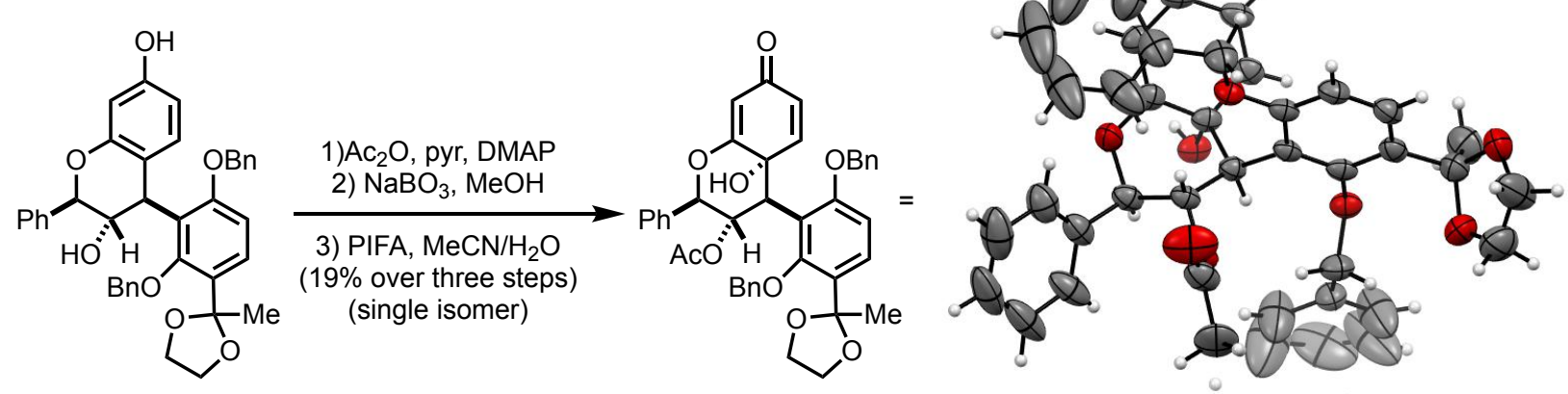

Note: Initially, the relative configuration of $\mathbf{1 6}$ was unknown and ambiguous by NOESY analysis. We confirmed the relative configuration of 16 by single crystal X-Ray analysis of $p$-quinol SI-7, which was made for a prior synthetic route. To synthesize SI-7, 16 was bis-acylated, and then the aryl acetate selectively removed with sodium perborate. Treatment of this material with phenyliodide(III) bis(trifluoroacetete) led to a single isolable compound, assigned unambiguously as compound SI-7. The characterization data for SI7 is below:

${ }^{1} \mathrm{H}$ NMR (400 MHz, Chloroform- $d$ ): $\delta 7.70(\mathrm{~d}, \mathrm{~J}=7.4,2 \mathrm{H}), 7.52-7.37(\mathrm{~m}, 9 \mathrm{H}), 7.37-$ $7.29(\mathrm{~m}, 3 \mathrm{H}), 7.25-7.20(\mathrm{~m}, 1 \mathrm{H}), 6.61(\mathrm{~d}, \mathrm{~J}-8.9 \mathrm{~Hz}, 1 \mathrm{H}), 6.63(\mathrm{~d}, \mathrm{~J}=9.9 \mathrm{~Hz}, 1 \mathrm{H}), 6.02$ $(\mathrm{dd}, \mathrm{J}=10.0,9.0 \mathrm{~Hz}, 1 \mathrm{H}), 5.79(\mathrm{dd}, \mathrm{J}-10.1,1.8,1 \mathrm{H}), 5.71(\mathrm{~d}, \mathrm{~J}=10.3,1 \mathrm{H}), 5.19(\mathrm{~d}, \mathrm{~J}=$ $1.8 \mathrm{~Hz}, 1 \mathrm{H}), 5.15(\mathrm{~d}, \mathrm{~J}=9.6 \mathrm{~Hz}, 1 \mathrm{H}), 5.03-4.90(\mathrm{~m}, 2 \mathrm{H}), 4.85(\mathrm{~d}, \mathrm{~J}=11.2,1 \mathrm{H}), 4.29(\mathrm{~d}$, $\mathrm{J}=8.9 \mathrm{~Hz}, 1 \mathrm{H}), 4.10-4.06(\mathrm{~m}, 2 \mathrm{H}), 3.99-3.79(\mathrm{~m}, 1 \mathrm{H}), 1.77(\mathrm{~s}, 3 \mathrm{H}), 1.61(\mathrm{~s}, 3 \mathrm{H}) .{ }^{13} \mathrm{C}$ NMR (151 MHz, $\left.\mathrm{CDCl}_{3}\right): \delta 187.7,172.2,169.0,158.5,156.5,143.1,137.8,136.4,136.1$, $129.2,129.1,128.9,128.8,128.7,128.7,128.7,128.6,128.4,128.4,128.3,128.2,128.1$, 128.0, 126.8, 119.6, 108.8, 107.6, 104.7, 100.2, 78.9, 71.7, 70.8, 69.9, 64.7, 64.6, 46.5, 26.4, 20.5. . IR (ATR): $v_{\max }\left(\mathrm{cm}^{-1}\right)=3342(\mathrm{br}), 3033(\mathrm{~m}), 2935(\mathrm{~m}), 2885(\mathrm{~m}) \mathrm{m} 1749(\mathrm{~s})$, 1663 (s), 1594 (s), 1421 (s), 1191 (vs), 1074 (s), 959 (m), 861 (m), 698 (s). HRMS (ESI+) Calcd. for $\mathrm{C}_{41} \mathrm{H}_{38} \mathrm{NaO}_{9}$. [M+Na] $]^{+}$: 697.2414. found: 697.2407. TLC (EtOAc-hexanes = $1: 1): R_{\mathrm{f}}=0.37$. 

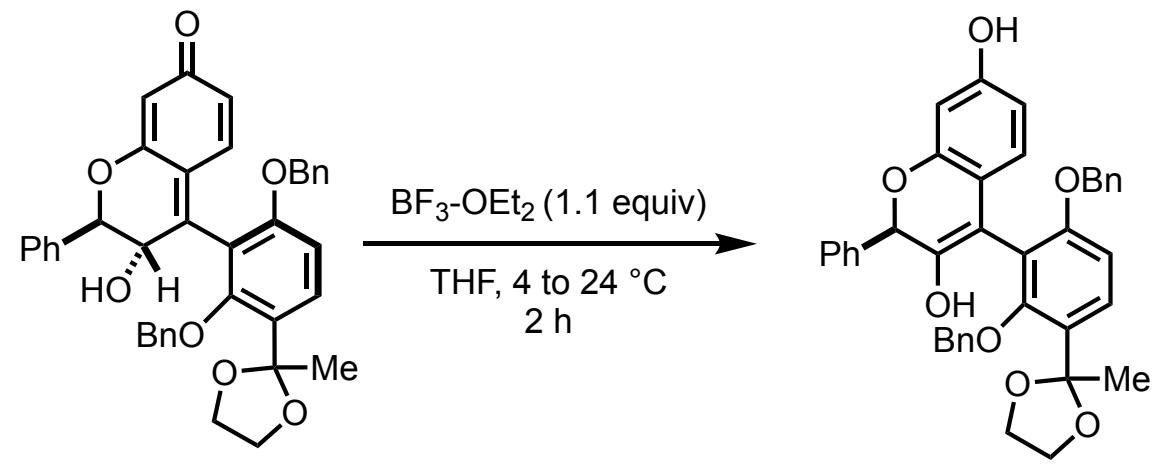

\section{Enol 13}

To a solution of quinone methide $14(220 \mathrm{mg}, 0.35 \mathrm{mmol})$ in THF $(8 \mathrm{~mL})$ at $4{ }^{\circ} \mathrm{C}$ was added boron trifluoride diethyl etherate $(51 \mu \mathrm{L}, 0.39 \mathrm{mmol})$. The solution stirred at 4 ${ }^{\circ} \mathrm{C}$ for 15 mins before being warmed slowly to room temperature over the course of 30 minutes. After $2 \mathrm{~h}$, saturated sodium acetate $(10 \mathrm{~mL})$ was added, followed by DCM. The contents were transferred to a separatory funnel and an additional $10 \mathrm{~mL}$ DCM added. The aqueous phase was extracted with DCM $(3 \times 5 \mathrm{~mL})$ and the combined organics washed with saturated brine $(2 \times 10 \mathrm{~mL})$ before being dried over $\mathrm{Na}_{2} \mathrm{SO}_{4}$, filtered, and concentrated in vacuo. The light purple foam was sensitive to oxygen and this was roughly characterized in crude form and presumably as a 1:1.1 mixture of atropisomers.

${ }^{1} \mathrm{H}$ NMR (600 MHz, Chloroform- $d$ ): 7.51 (dd, $\left.J=8.6,3.9 \mathrm{~Hz}, 2 \mathrm{H}\right), 7.37-7.32(\mathrm{~m}, 2 \mathrm{H})$, $7.33-7.29(\mathrm{~m}, 2 \mathrm{H}), 7.20-7.05(\mathrm{~m}, 12 \mathrm{H}), 7.00(\mathrm{dt}, J=22.7,7.7 \mathrm{~Hz}, 4 \mathrm{H}), 6.94-6.86(\mathrm{~m}$, $5 \mathrm{H}), 6.79(\mathrm{~d}, J=8.7 \mathrm{~Hz}, 1 \mathrm{H}), 6.74(\mathrm{~d}, J=8.7 \mathrm{~Hz}, 1 \mathrm{H}), 6.52(\mathrm{dd}, J=8.3,4.6 \mathrm{~Hz}, 2 \mathrm{H}), 6.45$ (d, $J=2.5 \mathrm{~Hz}, 1 \mathrm{H}$ ), 6.27 (d, $J=2.5 \mathrm{~Hz}, 1 \mathrm{H}), 6.22$ (ddd, $J=15.1,8.3,2.5 \mathrm{~Hz}, 2 \mathrm{H}), 5.78$ (d, $J=8.6 \mathrm{~Hz}, 2 \mathrm{H}), 5.54(\mathrm{~s}, 1 \mathrm{H}), 5.37(\mathrm{~s}, 1 \mathrm{H}), 4.98(\mathrm{~s}, 1 \mathrm{H}), 4.97$ ? $4.84(\mathrm{~m}, 3 \mathrm{H}), 4.77(\mathrm{~d}, J=$ $9.8 \mathrm{~Hz}, 1 \mathrm{H}), 4.68(\mathrm{~d}, J=9.9 \mathrm{~Hz}, 1 \mathrm{H}), 4.59(\mathrm{~d}, J=9.7 \mathrm{~Hz}, 1 \mathrm{H}), 4.06-3.95(\mathrm{~m}, 4 \mathrm{H}), 3.91$ $-3.73(\mathrm{~m}, 4 \mathrm{H}), 1.73-1.68(\mathrm{~m}, 5 \mathrm{H}) .{ }^{13} \mathrm{C}$ NMR (151 MHz, CDCl 3$)$ 158.1, 157.9, 156.3, 155.8, 154.7, 154.5, 152.3, 151.2, 145.5, 144.4, 139.5, 138.2, 137.5, 136.9, 136.7, 136.4, $129.9,129.4,128.6,128.5,128.5,128.4,128.4,128.3,128.2,128.2,128.0,127.9,127.9$, 127.9, 127.8, 127.6, 127.5, 127.3, 126.7, 126.6, 125.1, 125.0, 117.6, 117.4, 117.0, 116.7, 108.8, 108.7, 108.5, 108.4, 108.3, 107.9, 103.7, 103.7, 103.7, 102.5, 78.1, 77.8, 76.6, 76.3, 70.6, 70.3, 64.6, 64.6, 64.6, 64.5, 26.4, 26.4. TLC (EtOAc-hexanes $=1: 1): R_{\mathrm{f}}=$ 0.56 .

Note: attempts at purifying 13 by silica gel column chromatography led to significant losses. 
<smiles>CCC1(c2ccc(OCc3ccccc3)c(C3=C(O)C(c4ccccc4)Oc4cc(O)ccc43)c2O)OCCO1</smiles>

13

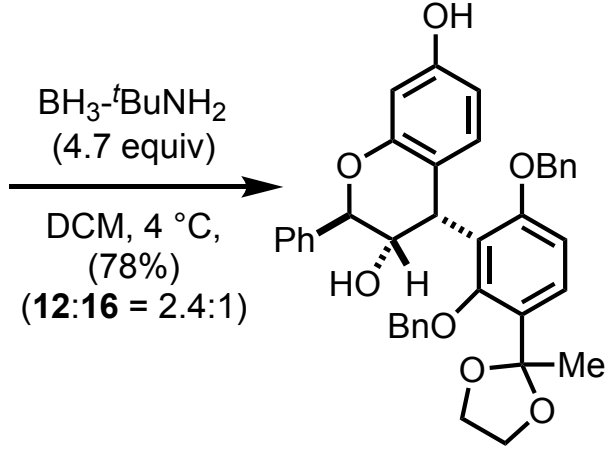

12<smiles>CC(C)(C)c1ccc(OCc2ccccc2)c(C2c3ccc(O)cc3OC(c3ccccc3)[C@H]2O)c1OC1(c2ccccc2)OCCO1</smiles>

16

\section{Alcohol 12}

To a solution of $13(502 \mathrm{mg}, 0.818 \mathrm{mmol})$ in $\mathrm{DCM}$ at $4{ }^{\circ} \mathrm{C}$ was added $\mathrm{BH}_{3}-{ }^{\mathrm{t}} \mathrm{BuNH}_{2}$ (4 portions over 3 hours, $336 \mathrm{mg}, 3.86 \mathrm{mmol}, 4.7$ equiv). The solution was stirred at $4{ }^{\circ} \mathrm{C}$ for 6 hours before being quenched with saturated aqueous ammonium chloride $(20 \mathrm{~mL})$. The contents transferred to a separatory funnel and aqueous phase washed with DCM $(4 \times 20 \mathrm{~mL})$. The combined organics washed with brine $(4 \times 20 \mathrm{~mL})$, dried over $\mathrm{Na}_{2} \mathrm{SO}_{4}$, filtered, and concentrated in vacuo. Crude ${ }^{1} \mathrm{H}$ NMR showed a 2.4:1 ratio of 12 and 16. The residue was dissolved in DCM (ca. $40 \mathrm{~mL}$ ), and silica gel added (ca $5 \mathrm{~g}$ ), before being concentrated to dryness and chromatographed directly (EtOAc-hexanes 10\% $\rightarrow 50 \%$ ). The fractions containing pure 12 were combined while the fractions containing a mixture of 12 and 16 combined and chromatographed again. This process was carried out three times to eventually yield $238.2 \mathrm{mg}$ pure $12(42 \%)$ and a ca. 1:2 mixture of 12 and 16 (153 $\mathrm{mg}$ )

${ }^{1} \mathrm{H}$ NMR (600 MHz, Chloroform- $d$ ), 12: $7.53(\mathrm{~d}, J=8.7 \mathrm{~Hz}, 1 \mathrm{H}), 7.29-7.27(\mathrm{~m}, 3 \mathrm{H})$, $7.25-7.12(\mathrm{~m}, 6 \mathrm{H}), 6.97-6.90(\mathrm{~m}, 2 \mathrm{H}), 6.87(\mathrm{dd}, J=6.6,2.9 \mathrm{~Hz}, 2 \mathrm{H}), 6.81(\mathrm{~d}, J=8.8$ $\mathrm{Hz}, 1 \mathrm{H}), 6.64-6.56(\mathrm{~m}, 1 \mathrm{H}), 6.52(\mathrm{~d}, J=2.6 \mathrm{~Hz}, 1 \mathrm{H}), 6.34(\mathrm{dd}, J=8.4,2.6 \mathrm{~Hz}, 1 \mathrm{H}), 5.42$ $(\mathrm{d}, J=3.1 \mathrm{~Hz}, 1 \mathrm{H}), 4.94(\mathrm{~d}, J=11.1 \mathrm{~Hz}, 1 \mathrm{H}), 4.82-4.58(\mathrm{~m}, 5 \mathrm{H}), 4.52-4.38(\mathrm{~m}, 2 \mathrm{H})$, $4.07(\mathrm{td}, J=4.9,4.0,2.2 \mathrm{~Hz}, 2 \mathrm{H}), 3.99-3.85(\mathrm{~m}, 2 \mathrm{H}), 1.74(\mathrm{~s}, 3 \mathrm{H}) .{ }^{13} \mathrm{C}$ NMR (151 MHz, $\left.\mathrm{CDCl}_{3}\right): 157.8,156.9,155.2,154.3,139.3,136.9,135.3,130.1,128.7,128.7,128.5$, 128.2, 128.1, 128.1, 127.6, 127.5, 127.2, 127.2, 125.2, 123.7, 115.3, 108.7, 108.7, 108.2, 103.2, 81.3, 77.1, 72.1, 71.1, 64.4, 64.4, 33.4, 26.1. IR (ATR): $v_{\max }\left(\mathrm{cm}^{-1}\right)=3307(\mathrm{~m})$, $3246(\mathrm{~m}), 2924(\mathrm{~m}), 2888(\mathrm{~m}), 1622(\mathrm{~s}), 1510(\mathrm{~m}), 1455(\mathrm{~s}), 1388(\mathrm{~m}), 1208(\mathrm{vs}), 1170$ (vs), 1081 (vs), 1000 (m), 847 (s), 695 (s). HRMS (ESI+) Calcd. for $\mathrm{C}_{39} \mathrm{H}_{36} \mathrm{NaO}_{7}$. [M+Na] ${ }^{+}$: 639.2359. found: 639.2349 TLC (EtOAc-hexanes $=1: 1): R_{f}=0.43$. 
<smiles>CC(C)(C)c1ccc(C2(C)OCCO2)c([C@@H]2c3ccc(O)cc3OC(c3ccccc3)[C@H]2O)c1O</smiles>
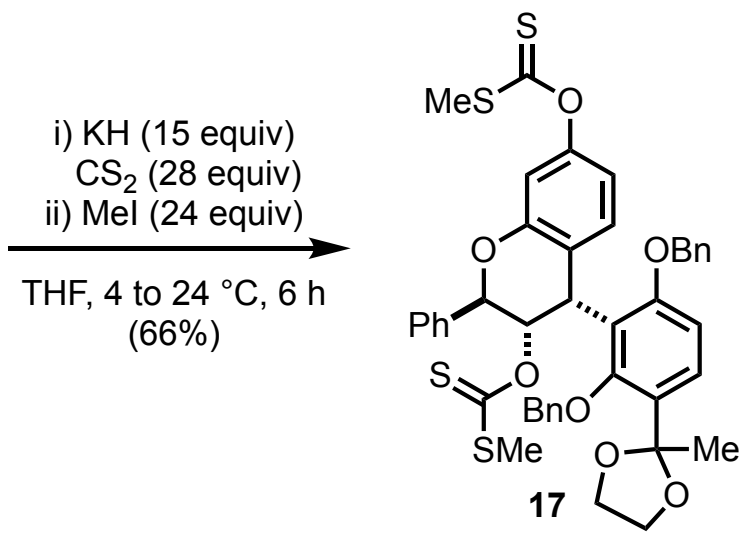

\section{Xanthate 17}

To a stirred solution of $\mathrm{KH}(30 \%$ in oil, $333 \mathrm{mg}, 2.55 \mathrm{mmol})$ in THF $(2.9 \mathrm{~mL})$ at 4 ${ }^{\circ} \mathrm{C}$ was added a solution of alcohol $16(105 \mathrm{mg}, 0.170 \mathrm{mmol})$ in THF $(6 \mathrm{~mL})$. The mixture was warmed to $24{ }^{\circ} \mathrm{C}$ and stirred for 40 min before being cooled back down to $4{ }^{\circ} \mathrm{C}$. Carbon disulfide $(213 \mu \mathrm{L}, 3.02 \mathrm{mmol})$ was added and the mixture was stirred for $1 \mathrm{~h}$ at 4 ${ }^{\circ} \mathrm{C}$ before being warmed to $24{ }^{\circ} \mathrm{C}$ for $2 \mathrm{~h}$ before an additional $120 \mu \mathrm{L} \mathrm{CS} 2$ was added. After $7 \mathrm{~h}$ the reaction was then cooled to $4{ }^{\circ} \mathrm{C}$ and $\mathrm{Mel}(414 \mu \mathrm{L}, 6.04 \mathrm{mmol})$ was added and the reaction stirred for 30 min before being quenched by addition of saturated sodium bibcarbonate $(10 \mathrm{~mL})$ and diluted with EtOAc $(10 \mathrm{~mL}$. The suspension was extracted with EtOAc $(5 \times 10 \mathrm{~mL})$, combined organic extracts washed with brine $(20 \mathrm{~mL})$. The combined organic extracted were then dried over $\mathrm{Na}_{2} \mathrm{SO}_{4}$, filtered, and evaporated in vacuo. The residue was chromatographed (EtOAc-hexanes 1:10 --> 1:2) to give 17 as a yellow oil $80.5 \mathrm{mg}, 66 \%$ yield)

${ }^{1} \mathrm{H}$ NMR (600 MHz, Chloroform- $d$ ): 7.54 (d, $\left.J=8.7 \mathrm{~Hz}, 1 \mathrm{H}\right), 7.37-7.20(\mathrm{~m}, 14 \mathrm{H}), 7.09$ $-7.03(\mathrm{~m}, 2 \mathrm{H}), 6.97(\mathrm{dd}, J=8.4,1.1 \mathrm{~Hz}, 1 \mathrm{H}), 6.77(\mathrm{~d}, J=8.8 \mathrm{~Hz}, 1 \mathrm{H}), 6.73(\mathrm{~d}, J=2.4$ $\mathrm{Hz}, 1 \mathrm{H}), 6.66-6.62(\mathrm{~m}, 2 \mathrm{H}), 5.57(\mathrm{~d}, J=6.3 \mathrm{~Hz}, 1 \mathrm{H}), 5.17-5.08(\mathrm{~m}, 2 \mathrm{H}), 4.98(\mathrm{~d}, J=$ $11.5 \mathrm{~Hz}, 1 \mathrm{H}), 4.80(\mathrm{dd}, J=10.9,4.8 \mathrm{~Hz}, 2 \mathrm{H}), 4.16-4.05(\mathrm{~m}, 2 \mathrm{H}), 4.04-3.96(\mathrm{~m}, 1 \mathrm{H})$, $3.96-3.88(\mathrm{~m}, 1 \mathrm{H}), 2.70(\mathrm{~s}, 3 \mathrm{H}), 2.25(\mathrm{~s}, 3 \mathrm{H}), 1.82(\mathrm{~s}, 3 \mathrm{H}) .{ }^{13} \mathrm{C} \mathrm{NMR}\left(151 \mathrm{MHz}, \mathbf{C D C l}_{3}\right)$ : 215.5, 214.8, 158.7, 156.9, 154.6, 153.6, 137.6, 137.3, 136.4, 128.9, 128.6, 128.6, 128.6, 128.4, 128.3, 128.3, 127.9, 127.8, 127.7, 127.6, 127.5, 126.5, 122.8, 121.7, 114.7, 110.7, 108.9, 108.1, 79.7, 77.0, 70.6, 64.5, 64.4, 33.8, 26.3, 20.1, 18.6. IR (ATR): $v_{\max }\left(\mathrm{cm}^{-1}\right)=$ $2922(\mathrm{~m}), 2882(\mathrm{~m}), 1592(\mathrm{~s}), 1494$ (s), $1421(\mathrm{~m}), 1253$ (vs), 1131 (s), 1060 (vs), 1041 (s), $979(\mathrm{~m})$. HRMS (ESI+) Calcd. for $\mathrm{C}_{43} \mathrm{H}_{40} \mathrm{O}_{7} \mathrm{~S}_{4} \mathrm{Na}$. [M+Na] ${ }^{+}$: 819.1555, found: 819.1545. TLC (EtOAc-hexanes $=1: 10,3$ developments): $R_{\mathrm{f}}=0.47$. 


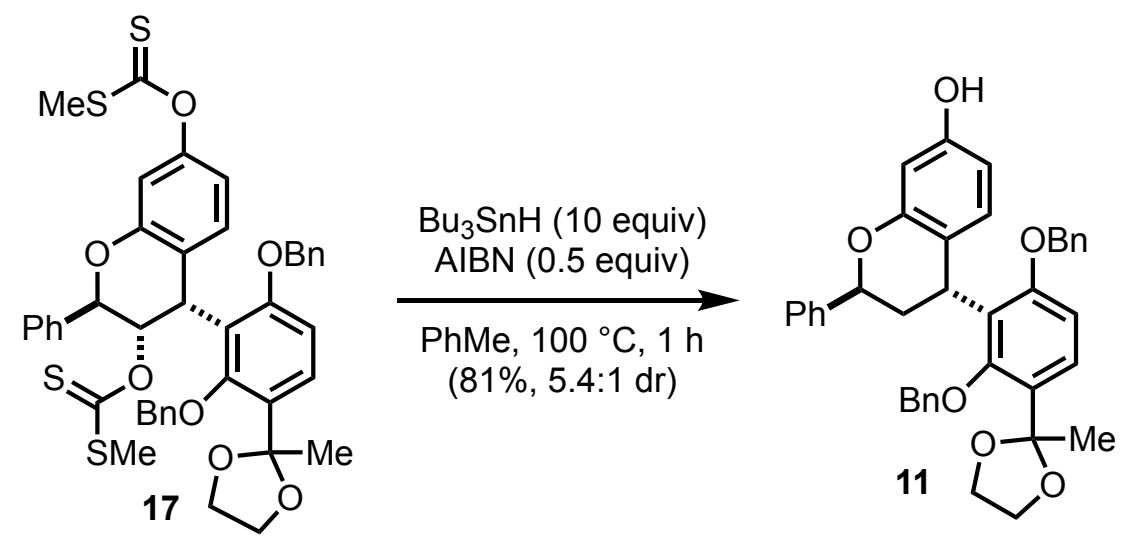

\section{Phenol 11}

To a solution of xanthate $17(80.5 \mathrm{mg}, 0.10 \mathrm{mmol})$ in degassed $\mathrm{PhMe}(4 \mathrm{~mL})$ was added $\mathrm{Bu}_{4} \mathrm{SnH}(136 \mu \mathrm{L}, 0.5 \mathrm{mmol})$ and AIBN $(7.9 \mathrm{mg}, 0.044 \mathrm{mmol})$, consecutively. The solution was then heated to $100{ }^{\circ} \mathrm{C}$ for 3 hours before being cooled to $24{ }^{\circ} \mathrm{C}$ and concentrated in vacuo. The resulting oily residue was chromatographed directly (hexanes $\rightarrow 50 \%$ EtOAc-hexanes) to give phenol 11 (44.6 mg, 81\%, 5.4:1 dr) as a colorless foam. ${ }^{1} \mathrm{H}$ NMR (600 MHz, Chloroform- $d$ ): $7.51-7.42(\mathrm{~m}, 1 \mathrm{H}), 7.34-7.17(\mathrm{~m}, 12 \mathrm{H}), 7.05-$ $6.97(\mathrm{~m}, 2 \mathrm{H}), 6.91(\mathrm{~d}, J=7.2 \mathrm{~Hz}, 2 \mathrm{H}), 6.79-6.68(\mathrm{~m}, 2 \mathrm{H}), 6.57(\mathrm{~d}, J=8.3 \mathrm{~Hz}, 1 \mathrm{H}), 6.28$ (ddd, $J=13.6,8.3,2.6 \mathrm{~Hz}, 1 \mathrm{H}), 5.54(\mathrm{t}, J=4.1 \mathrm{~Hz}, 1 \mathrm{H}), 4.99-4.84(\mathrm{~m}, 2 \mathrm{H}), 4.82-4.61$ (m, 4H), $4.51(\mathrm{dd}, J=11.1,5.8 \mathrm{~Hz}, 1 \mathrm{H}), 4.14-4.06(\mathrm{~m}, 3 \mathrm{H}), 4.00-3.87(\mathrm{~m}, 3 \mathrm{H}), 3.06$ (ddd, $J=13.3,10.8,4.7 \mathrm{~Hz}, 1 \mathrm{H}$ ), 2.29 (ddd, $J=13.5,5.9,3.5 \mathrm{~Hz}, 1 \mathrm{H}), 1.79(\mathrm{~s}, 3 \mathrm{H}) .{ }^{13} \mathrm{C}$ NMR (151 MHz, $\left.\mathbf{C D C l}_{3}\right)$ : 158.4, 156.3, 155.0, 154.9, 142.0, 137.2, 136.7, 128.7, 128.6, 128.3, 128.3, 128.2, 127.8, 127.7, 127.2, 127.1, 126.2, 125.4, 119.1, 109.0, 108.1, 107.7, 103.2, 75.8, 70.1, 64.5, 32.4, 27.7, 26.3. note: 2 overlapping aliphatic Cs. IR (ATR): $v_{\max }$ $\left(\mathrm{cm}^{-1}\right)=3389(\mathrm{br}), 3031(\mathrm{~m}), 2934(\mathrm{~m}), 2880(\mathrm{~m}), 1591(\mathrm{~s}), 1503(\mathrm{~s}), 1478(\mathrm{~s}), 1304(\mathrm{~m})$, 1263 (s), 1209 (s), 1152 (s), 1041 (s), 965 (m), 842 (m). HRMS (ESI+) Calcd. for $\mathrm{C}_{39} \mathrm{H}_{36} \mathrm{NaO}_{6}$. $[\mathrm{M}+\mathrm{Na}]^{+}:$623.2410. Found: 623.2407. TLC (EtOAc-hexanes $\left.=1: 3\right): R_{\mathrm{f}}=$ 0.29 . 
<smiles>CC(=O)Oc1ccc2c(c1)O[C@H](c1ccccc1)C=C2c1c(OCc2ccccc2)ccc(C2(C)OCCO2)c1OCc1ccccc1</smiles>
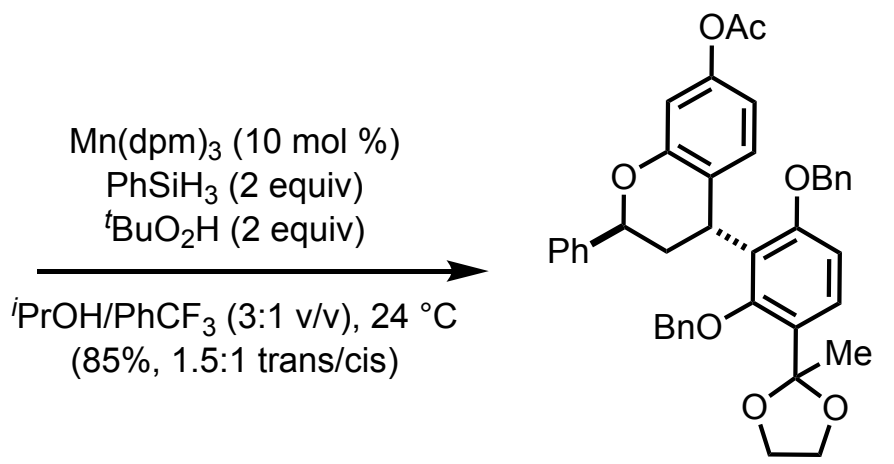<smiles>CC(=O)Oc1ccc2c(c1)O[C@@H](c1ccccc1)C[C@H]2c1c(OCc2ccccc2)ccc(C2(C)OCCO2)c1OCc1ccccc1</smiles>

\section{Dihydropyrans 10a and 10b:}

To a stirred solution of 9 (200 mg, $0.312 \mathrm{mmol}, 1$ equiv) in dry $\mathrm{PhCF}_{3} / \mathrm{PrOH}(8.0 \mathrm{~mL}, 3: 1$ $\mathrm{v} / \mathrm{v}$ ) was added $\mathrm{PhSiH}_{3}(67 \mu \mathrm{L}, 0.624 \mathrm{mmol}, 2$ equiv) and tbutyl hydroperoxide $(220 \mu \mathrm{L}$, $5.5 \mathrm{M}$ in decane, $1.21 \mathrm{mmol}, 3.9$ equiv). The solution was sparged for 5 minutes with dry argon before $\mathrm{Mn}(\mathrm{dpm})_{3}(38.0 \mathrm{mg}, 0.062 \mathrm{mmol}, 0.2$ equiv) was added in one portion. The solution was then sparged for 10 seconds with dry argon before being left under a static atmosphere of argon and stirred at $24{ }^{\circ} \mathrm{C}$ for $19 \mathrm{~h}$. After completion (as judged by ESIMS), silica gel (ca. $2.5 \mathrm{~g}$ ) was added and the suspension concentrated to a free-flowing powder, which was chromatographed directly by MPLC $(12 \mathrm{~g}$ column, $20 \mathrm{~mL} / \mathrm{min}, 10 \%$ $\rightarrow 40 \%$ ethyl acetate/hexane) to give $10 \mathrm{a}$ and $10 \mathrm{~b}$ (170 $\mathrm{mg}, 85 \%)$ as a $1.5: 1$ mixture of trans/cis isomers and as a colorless oil. The mixture was separated by iterative, preparative reverse-phase HPLC $\left(21.2 \times 100 \mathrm{~mm}\right.$ Phenomenex Gemini column (5 $\mu \mathrm{m} \mathrm{C}_{18}$ $110 \AA, 21.2 \times 100 \mathrm{~mm}$ ), $65 \% \mathrm{MeCN}$ in $\mathrm{H}_{2} \mathrm{O} \rightarrow 100 \% \mathrm{MeCN}$ over 15 minutes, $20 \mathrm{~mL} / \mathrm{min}$, ca. $30 \mathrm{mg}$ injections) to give $10 \mathrm{a}(41 \mathrm{mg})$ and $10 \mathrm{~b}(39 \mathrm{mg})$, both as colorless foams $(47 \%$ HPLC recovery). The relative stereochemistry of $10 \mathrm{a}$ and $10 \mathrm{~b}$ was assigned both by analogy to similar compounds and NOESY analysis (Figures S1 and S2). ${ }^{\mathrm{S}}$

Note 1: In most cases, the reaction stalled at partial conversion. Due our inability to separate 9 and $10 \mathrm{a} / 10 \mathrm{~b}$ by silica gel chromatography, an additional portion of $\mathrm{PhSiH}_{3}$, TBHP, and catalyst were added. In each case, this restarted the reaction and led to full conversion.

Note 2: Preparative HPLC recovery ranged from ca. $40 \%$ to $70 \%$ based on injection size.

10a: ${ }^{1} \mathrm{H}$ NMR (600 MHz, Chloroform- $\left.d\right)$ : $\delta 7.47(\mathrm{~d}, J=8.6 \mathrm{~Hz}, 1 \mathrm{H}), 7.30-7.20(\mathrm{~m}, 13 \mathrm{H})$, $7.03(\mathrm{~d}, J=7.0 \mathrm{~Hz}, 2 \mathrm{H}), 6.88(\mathrm{~d}, J=6.9 \mathrm{~Hz}, 2 \mathrm{H}), 6.75(\mathrm{~d}, J=2.4 \mathrm{~Hz}, 1 \mathrm{H}), 6.74-6.70(\mathrm{~m}$, 2H), 6.52 (dd, $J=8.4,2.4 \mathrm{~Hz}, 1 \mathrm{H}), 5.51(\mathrm{t}, J=4.2 \mathrm{~Hz}, 1 \mathrm{H}), 4.88(\mathrm{~d}, J=11.4 \mathrm{~Hz}, 1 \mathrm{H}), 4.76$ (dd, $J=18.7,10.4 \mathrm{~Hz}, 2 \mathrm{H}), 4.72-4.63(\mathrm{~m}, 1 \mathrm{H}), 4.55(\mathrm{dd}, J=10.5,6.0 \mathrm{~Hz}, 1 \mathrm{H}), 4.15-$ $4.04(\mathrm{~m}, 2 \mathrm{H}), 4.00-3.88(\mathrm{~m}, 2 \mathrm{H}), 3.00$ (ddd, $J=14.4,10.4,4.6 \mathrm{~Hz}, 1 \mathrm{H}), 2.36-2.24(\mathrm{~m}$,

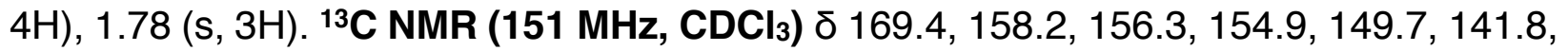
137.2, 136.5, 128.7, 128.6, 128.5, 128.3, 127.8, 127.8, 127.3, 127.3, 126.8, 126.4, 125.4, 124.3, 113.4, 109.8, 109.0, 108.0, 100.1, 97.1, 77.2, 75.8, 70.3, 64.5, 64.5, 32.5, 28.1, 26.3, 21.3. IR (ATR): $v_{\max }\left(\mathrm{cm}^{-1}\right)=3031(\mathrm{w}), 2934(\mathrm{~m}), 2887(\mathrm{~m}), 2361(\mathrm{w}), 1752(\mathrm{~s})$, 
1583 (s), 1585 (s) ,1472 (m), 1370 (s), 1252 (w), 1209 (Vs), 1143 (s), 1110 (s), 1087 (s), $1081(\mathrm{~m}), 1043(\mathrm{~s}), 1016(\mathrm{~m}), 906(\mathrm{w}), 811(\mathrm{w}), 736(\mathrm{~m}), 698(\mathrm{~m}), 676(\mathrm{~m})$. HRMS (ESI+) Calcd. for $\mathrm{C}_{41} \mathrm{H}_{38} \mathrm{O}_{7} \mathrm{Na}$ (Both isomers). [M+Na] $]^{+}$665.2515. Found: 665.2509. TLC (EtOAc-hexanes $=50: 50$ ): both isomers, $R_{\mathrm{f}}=0.50$

10b: ${ }^{1} \mathrm{H}$ NMR (600 MHz, Chloroform- $\left.d\right)$ : $\delta 7.60-7.54(\mathrm{~m}, 2 \mathrm{H}), 7.48(\mathrm{~d}, J=8.6 \mathrm{~Hz}, 1 \mathrm{H})$, $7.43(\mathrm{t}, J=7.5 \mathrm{~Hz}, 2 \mathrm{H}), 7.39-7.35(\mathrm{~m}, 1 \mathrm{H}), 7.33-7.24(\mathrm{~m}, 7 \mathrm{H}), 7.23-7.16(\mathrm{~m}, 2 \mathrm{H})$, $6.99-6.91(\mathrm{~m}, 2 \mathrm{H}), 6.82(\mathrm{dd}, J=8.4,1.1 \mathrm{~Hz}, 1 \mathrm{H}), 6.71(\mathrm{~d}, J=8.7 \mathrm{~Hz}, 1 \mathrm{H}), 6.63(\mathrm{~d}, J=$ $2.4 \mathrm{~Hz}, 1 \mathrm{H}$ ), 6.54 (dd, $J=8.4,2.4 \mathrm{~Hz}, 1 \mathrm{H}), 5.09(\mathrm{~d}, J=10.4 \mathrm{~Hz}, 1 \mathrm{H}), 5.04$ (dd, $J=11.3$, $2.4 \mathrm{~Hz}, 2 \mathrm{H}), 4.92(\mathrm{dd}, J=11.9,5.7 \mathrm{~Hz}, 1 \mathrm{H}), 4.84(\mathrm{~d}, J=11.2 \mathrm{~Hz}, 1 \mathrm{H}), 4.75(\mathrm{~d}, J=11.2$ $\mathrm{Hz}, 1 \mathrm{H}), 4.20-4.07(\mathrm{~m}, 2 \mathrm{H}), 4.03-3.87(\mathrm{~m}, 2 \mathrm{H}), 2.77(\mathrm{~d}, J=11.8 \mathrm{~Hz}, 1 \mathrm{H}), 2.29(\mathrm{~s}, 3 \mathrm{H})$, 2.04 (ddd, $J=13.4,5.8,2.1 \mathrm{~Hz}, 1 \mathrm{H}), 1.88(\mathrm{~s}, 3 \mathrm{H}) .{ }^{13} \mathrm{C} \mathrm{NMR}\left(151 \mathrm{MHz}, \mathrm{CDCl}_{3}\right): \delta 169.5$, 158.0, 156.6, 155.8, 149.5, 141.5, 137.8, 136.5, 128.7, 128.7, 128.5, 128.3, 128.2, 128.1, 127.9, 127.8, 127.8, 127.4, 126.4, 126.4, 126.2, 124.0, 113.5, 110.0, 109.0, 107.8, 78.8, 77.8, 70.1, 64.6, 64.5, 35.0, 33.8, 26.5, 21.3. HRMS (ESI+) Calcd. for $\mathrm{C}_{41} \mathrm{H}_{38} \mathrm{O}_{7} \mathrm{Na}$ (Both isomers). $[\mathrm{M}+\mathrm{Na}]^{+}:$665.2515. Found: 665.2509. TLC (EtOAc-hexanes = 1:1): both isomers, $R_{\mathrm{f}}=0.50$ 
Figure S1: NOESY analysis of 10a.
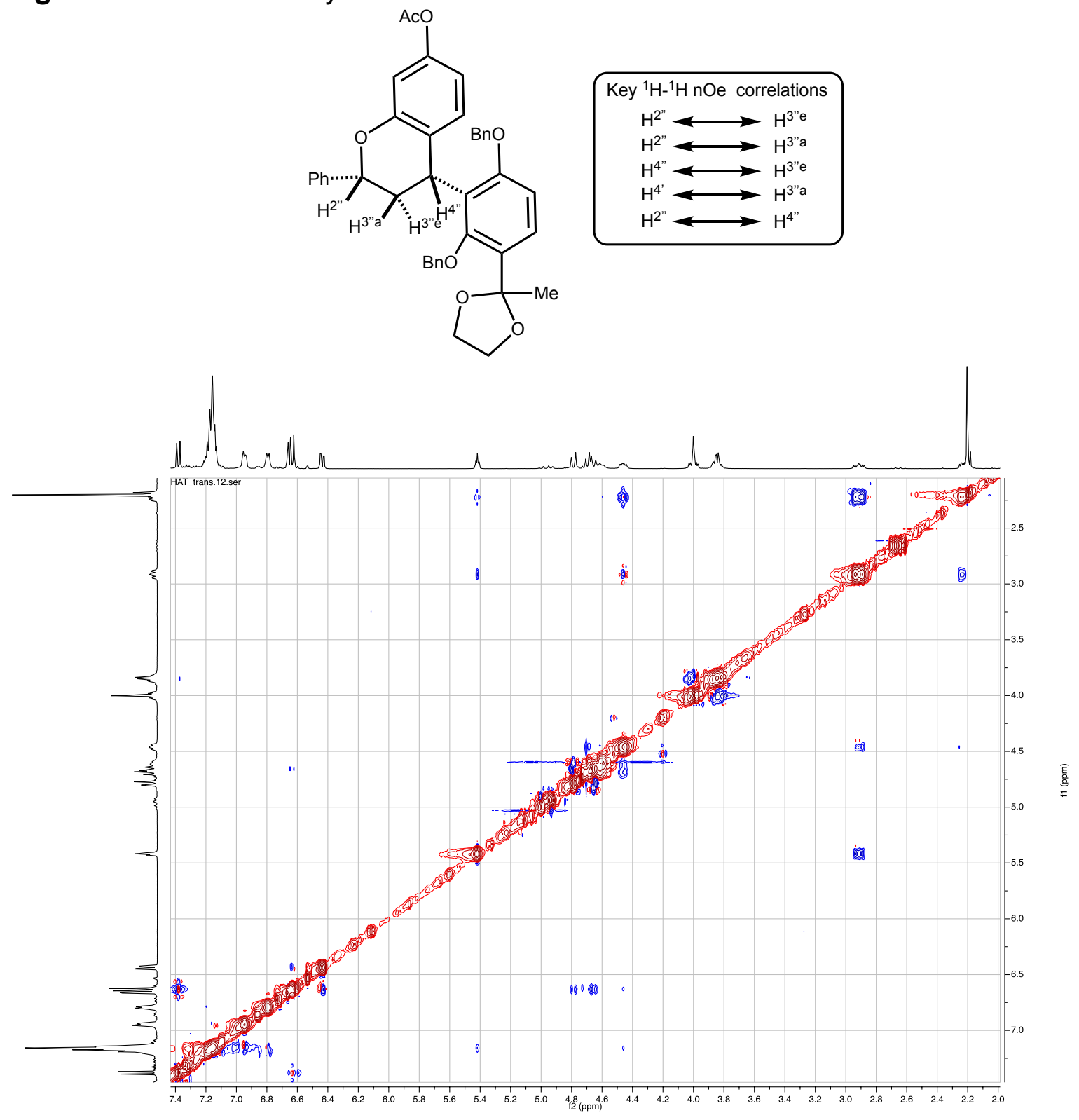
Figure S2: NOESY analysis of 10b.
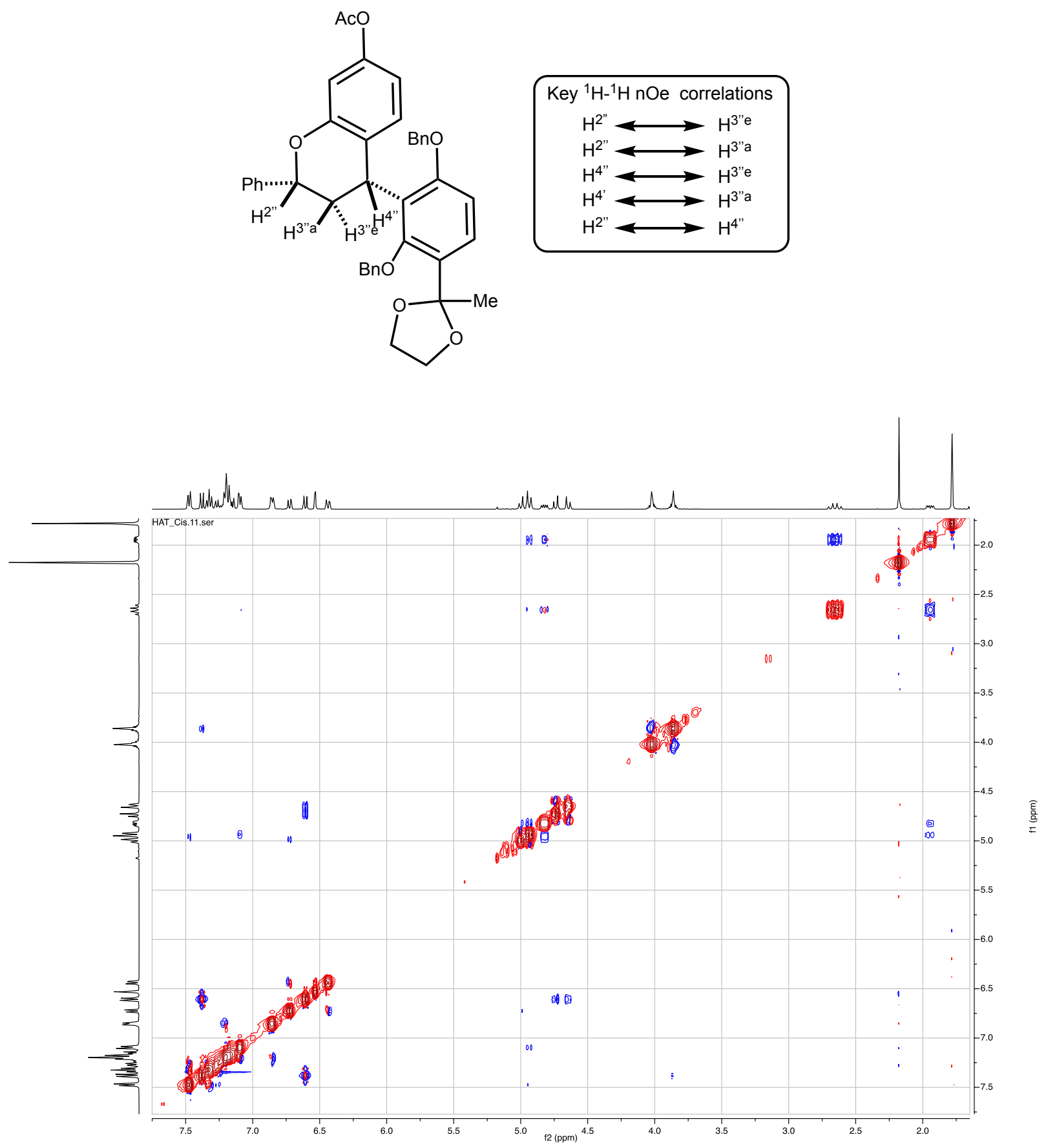


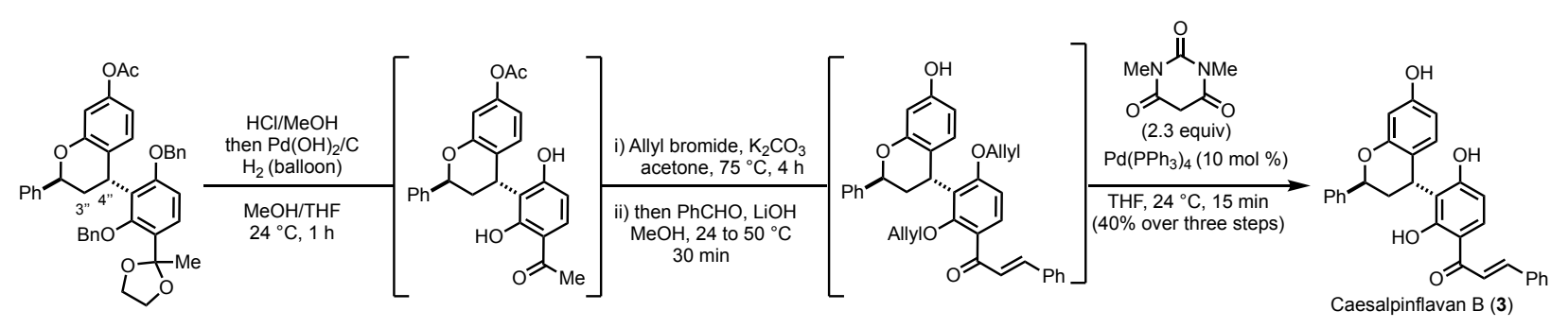

Caesalpinflavan B (3):

Step 1. To a solution of dihydropyran 10 a $(19.1 \mathrm{mg}, 0.029 \mathrm{mmol})$ in $\mathrm{MeOH}(1.5$ $\mathrm{mL}$ ) was added $10 \%$ aq. $\mathrm{HCl}(5 \mu \mathrm{L})$. The solution stirred at $24{ }^{\circ} \mathrm{C}$ for 15 minutes before being sparged with dry argon for 10 minutes, followed by evacuation under vacuum (ca. 15 torr) for 5 minutes. THF ( $1.5 \mathrm{~mL}$ ) was then added and the solution then sparged with hydrogen gas for 5 minutes before being placed under a static atmosphere of hydrogen gas (Note 1). To the resulting solution was added Pearlman's catalyst (11.2 mg, Note 2). The resulting suspension was sparged with hydrogen gas for 5 minutes before being placed under an atmosphere of static hydrogen and stirred vigorously for 75 minutes. After the reaction was complete, it was rapidly filtered through a plug of Celite, eluting with ethyl acetate $(5 \mathrm{~mL})$. The resulting solution was concentrated under vacuum to give bisphenol 18a (35.7 mg), which was used crude in the next step.

Step 2. To a solution of crude bisphenol 18 a $(35.7 \mathrm{mg})$ in acetone $(0.5 \mathrm{~mL})$ in a 10 $\mathrm{mL}$ microwave tube was added allyl bromide $(10.6 \mu \mathrm{L}, 0.12 \mathrm{mmol})$ and potassium carbonate $12.6 \mathrm{mg}, 0.091 \mathrm{mmol})$. The tube was then sealed and placed under an atmosphere of dry nitrogen before being heated in a microwave at $85^{\circ} \mathrm{C}$ for 3 hours. The suspension was then cooled to $24{ }^{\circ} \mathrm{C}$ and volatiles removed under vacuum (Note 3). $\mathrm{MeOH}(0.5 \mathrm{~mL})$ was then added to the tube and the suspension stirred for 10 minutes before benzaldehyde (12.5 $\mu \mathrm{L}, 0.12 \mathrm{mmol}$ ) and lithium hydroxide monohydrate (10 $\mathrm{mg}$, $0.24 \mathrm{mmol}$ ) were added. The tube was then heated in an aluminum heating block at 50 ${ }^{\circ} \mathrm{C}$ for 30 minutes. After being cooled to $24^{\circ} \mathrm{C}$, the suspension was quenched with $5 \mathrm{~mL}$ saturated aqueous ammonium chloride. The mixture was extracted with ethyl acetate (5 $x 5 \mathrm{~mL}$ ) and the combined organic phases washed with saturated brine $(5 \mathrm{~mL})$, dried over sodium sulfate, filtered, and concentrated in vacuo (Note 4). After drying under high vacuum overnight, the crude phenol 19a $(16.1 \mathrm{mg}$ ) was used crude in the next step (Note 5).

Step 3. To a dry $20 \mathrm{~mL}$ vial containing 1,3-dimethyl barbituric acid (11.2 mg, 0.072 $\mathrm{mmol}),\left[\mathrm{Pd}\left(\mathrm{PPh}_{3}\right)_{4}\right](3.4 \mathrm{mg}, 0.0029 \mathrm{mmol})$, crude phenol 19a $(14.7 \mathrm{mg})$ and a magnetic stir-bar was added dry THF $(4.5 \mathrm{~mL})$. The bright yellow solution was stirred for 15 mins before being rapidly filtered through a short plug of silica gel, which was eluted with ethyl acetate $(10 \mathrm{~mL})$. The resulting solution as concentrated under vacuum and purified by reverse-phase preparative TLC (water-acetonitrile $=1: 7$ ) followed by normal-phase preparative TLC $(5 \% \mathrm{MeOH}$ in $\mathrm{DCM})$. The yellow band was collected affording caesalpinflavan B (4.9 mg, $40 \%$ over 3 steps) as a yellow amorphous solid. 
Note 1: The sparging/vacuum cycle is to degas $\mathrm{HCl}$ from the reaction in order to avoid over reduction of the flavan $\mathrm{C}$ ring $\mathrm{C}-\mathrm{O}$ bond.

Note 2: We observed a marked effect of the age of Pearlman's catalyst and selectivity for our desired reaction; Fresh bottles of Pearlman's catalyst (from Millipore Sigma) gave mixtures of the desired bisphenol and overreduction, presumably to a trisphenol where the $\mathrm{C}-\mathrm{O}$ bond in the dihydropyran ring had been cleaved. Older bottles (ca. 15-20 years old, Aldrich) were less active and were slower to cleave this more hindered $\mathrm{C}-\mathrm{O}$ bond. However, if the reaction is not filtered through Celite quickly after the removal of both benzyl groups, $\mathrm{C}-\mathrm{O}$ bond cleavage of the flavanoid C-ring begins to occur (as judged by MS analysis of aliquots).

Note 3: It is imperative that the reaction mixture is thoroughly evacuated. If traces of allyl bromide remain during the saponification step, a compound with all three phenols protected as the aryl allyl ethers was obtained in ca. $50 \%$ yield along with the desired compound (ca. $40 \%$ yield). This was accomplished by evacuating the reaction flask for 2 hours under high vacuum (ca. 10 mTorr).

Note 4: High vacuum was ca. 200 mTorr for 12 hours. This removed the majority of remaining benzaldehyde from the Claisen-Schmidt reaction (as judged by ${ }^{1} \mathrm{H}$ NMR)

Note 5: The crude mixture contained ca. $20 \%$ triallyl compound as judged by UPLC/MS analysis.

${ }^{1} \mathrm{H}$ NMR $\left(600 \mathrm{MHz}, \mathrm{MeOH}-d_{4}\right): \delta 7.94(\mathrm{~d}, J=8.9 \mathrm{~Hz}, 1 \mathrm{H}), 7.85-7.79(\mathrm{~m}, 2 \mathrm{H}), 7.76-$ $7.71(\mathrm{~m}, 2 \mathrm{H}), 7.46-7.39(\mathrm{~m}, 3 \mathrm{H}), 7.38-7.33(\mathrm{~m}, 2 \mathrm{H}), 7.35-7.29(\mathrm{~m}, 3 \mathrm{H}), 7.25(\mathrm{t}, J=$ $7.2 \mathrm{~Hz}, 1 \mathrm{H}), 6.52(\mathrm{dd}, J=8.4,1.0 \mathrm{~Hz}, 1 \mathrm{H}), 6.46(\mathrm{~d}, J=8.9 \mathrm{~Hz}, 1 \mathrm{H}), 6.40(\mathrm{~d}, J=2.5 \mathrm{~Hz}$, $1 \mathrm{H}), 6.21(\mathrm{dd}, J=8.3,2.5 \mathrm{~Hz}, 1 \mathrm{H}), 5.54(\mathrm{dd}, J=5.8,3.9 \mathrm{~Hz}, 1 \mathrm{H}), 4.49(\mathrm{t}, J=7.3 \mathrm{~Hz}, 1 \mathrm{H})$, 2.74 (ddd, $J=13.6,8.3,3.9 \mathrm{~Hz}, 1 \mathrm{H}$ ), 2.25 (dt, $J=13.6,6.1 \mathrm{~Hz}, 1 \mathrm{H}$ ). ${ }^{13} \mathrm{C}$ NMR (151 MHz, MeOH-d $d_{4}: \delta 192.1,164.6,163.2,155.8,155.0,143.6,142.3,135.0,130.2,130.1,128.6$, 128.3, 128.1, 127.9, 126.7, 125.2, 120.5, 117.3, 116.8, 113.2, 107.5, 107.4, 102.4, 75.4, 32.9, 26.6.TLC $\left(\mathrm{C}_{18}\right.$ silica: water-acetonitrile $\left.=1: 7\right), R_{\mathrm{f}}=0.72$. IR (ATR): $v_{\max }\left(\mathrm{cm}^{-1}\right)=$ 3384 (br), 3061 (m), 3028 (w), $2925(\mathrm{~m}), 1631(\mathrm{~m}), 1588(\mathrm{vs}) 1503(3), 1449(3), 1345(\mathrm{~m})$, 1288 (s), 1228 (s), 1359 (s), 1156 (s), 1118 (m), 1027 (w), 977 (w), 759 (m), $697(\mathrm{~m})$. HRMS (ESI+) Calcd. for $\mathrm{C}_{30} \mathrm{H}_{24} \mathrm{O}_{5} \mathrm{Na}$ [M+Na]: 487.1521, found: 487.1516. Calcd. for $\mathrm{C}_{30} \mathrm{H}_{25} \mathrm{O}_{5}[\mathrm{M}+\mathrm{H}]:$ : 465.1702, found: 465.1700 . 


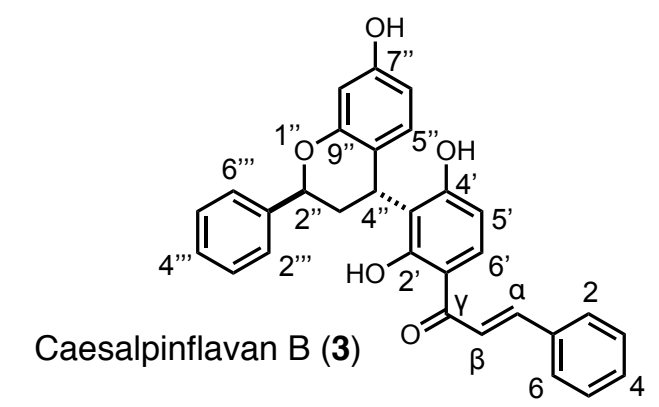

\begin{tabular}{|c|c|c|c|c|c|c|}
\hline \multirow[b]{2}{*}{ position } & \multicolumn{3}{|c|}{${ }^{1} \mathrm{H}$ NMR Chemical Shifts } & \multicolumn{3}{|c|}{${ }^{13} \mathrm{C}$ NMR Chemical Shifts } \\
\hline & $\begin{array}{c}\text { Natural } \\
(+) \text {-Caesalpinflavan B (3) }\end{array}$ & $\begin{array}{c}\text { Synthetic } \\
\text { ( } \pm \text {-Caesalpinflavan B (3) }\end{array}$ & $\Delta \mathrm{ppm}$ & $\begin{array}{c}\text { Natural } \\
(+) \text {-Caesalpinflavan B (3) }\end{array}$ & $\begin{array}{c}\text { Synthetic } \\
( \pm) \text {-Caesalpinflavan B (3) }\end{array}$ & $\Delta \mathrm{ppm}$ \\
\hline 1 & - & - & $\mathrm{n} / \mathrm{a}$ & 135.0 & 135.0 & - \\
\hline 2,6 & 7.76 (overlap) & 7.74 (overlap) & 0.02 & 128.3 & 128.3 & - \\
\hline 3,5 & 7.43 (overlap) & 7.43 (overlap) & - & 130.1 & 130.1 & - \\
\hline 4 & 7.43 (overlap) & 7.43 (overlap) & - & 128.7 & 128.7 & - \\
\hline a & 7.81 (overlap) & 7.82 (overlap) & 0.01 & 143.6 & 143.6 & - \\
\hline$b$ & 7.81 (overlap) & 7.82 (overlap) & 0.01 & 120.4 & 120.4 & - \\
\hline $\mathrm{y}$ & - & - & $\mathrm{n} / \mathrm{a}$ & 192.1 & 192.1 & - \\
\hline 1 ' & - & - & $\mathrm{n} / \mathrm{a}$ & not tabulated ${ }^{1}$ & 113.2 & $\mathrm{n} / \mathrm{a}$ \\
\hline $2^{\prime}$ & - & - & $\mathrm{n} / \mathrm{a}$ & 164.6 & 164.6 & - \\
\hline 3 & - & - & $\mathrm{n} / \mathrm{a}$ & 102.4 & 102.4 & - \\
\hline $4^{\prime}$ & - & - & $\mathrm{n} / \mathrm{a}$ & 163.2 & 163.2 & - \\
\hline 5 & $6.46(d, 9.0)$ & $6.46(d, 9.0)$ & - & 107.5 & 107.5 & - \\
\hline $6^{\prime}$ & $7.94(\mathrm{~d}, 9.0)$ & $7.94(\mathrm{~d}, 8.9)$ & - & 127.9 & 127.9 & - \\
\hline 2" & $5.53(\mathrm{dd}, 4.2,5.3)$ & $5.54(\mathrm{dd}, 5.8,3.9)$ & 0.01 & 75.4 & 75.4 & - \\
\hline 3" & 2.76 (ddd, 13.7, 8.4, 3.9) & 2.74 (ddd, 13.6, 8.3, 3.9) & 0.02 & 26.6 & 26.6 & - \\
\hline & $2.29(\mathrm{dt}, 13.7,6.0)$ & $2.25(\mathrm{dt}(13.6,6.1)$ & 0.04 & & & $\mathrm{n} / \mathrm{a}$ \\
\hline $4 "$ & $4.48(\mathrm{t}, 7.2)$ & $4.49(\mathrm{t}, 7.3)$ & 0.01 & 32.9 & 32.9 & - \\
\hline $5 "$ & $6.52(\mathrm{dd}, 8.4,0.8)$ & $6.52(\mathrm{~d}, 8.4,1.0)$ & - & 128.1 & 128.1 & - \\
\hline $6 "$ & $6.20(\mathrm{dd}, 8.4,2.5)$ & $6.21(8.3,2.5)$ & 0.01 & 107.4 & 107.4 & - \\
\hline $7 "$ & - & - & $\mathrm{n} / \mathrm{a}$ & 155.0 & 155.0 & - \\
\hline 8" & $6.39(\mathrm{~d}, 2.5)$ & $6.40(\mathrm{~d}, 2.5)$ & 0.01 & 102.4 & 102.4 & - \\
\hline $9 "$ & - & - & $\mathrm{n} / \mathrm{a}$ & 155.8 & 155.8 & - \\
\hline $10 "$ & - & - & $\mathrm{n} / \mathrm{a}$ & 117.3 & 117.3 & - \\
\hline $1 " ”$ & - & - & $\mathrm{n} / \mathrm{a}$ & 142.3 & 142.3 & - \\
\hline 2"',6”" & 7.36 (overlap) & 7.36 (overlap) & - & 125.2 & 125.2 & - \\
\hline 3"',5”' & 7.32 (overlap) & 7.32 (overlap) & - & 128.1 & 128.1 & - \\
\hline 4"” & $7.25(\mathrm{t}, 7.0)$ & $7.25(\mathrm{t}, 7.2)$ & - & 126.7 & 126.7 & - \\
\hline
\end{tabular}

Table S2. Comparison of ${ }^{1} \mathrm{H}$ chemical shifts and multiplicities between natural 3 (isolated by Wang and co-workers, ${ }^{56} 500 \mathrm{MHz}$ for ${ }^{1} \mathrm{H}, 125 \mathrm{MHz}$ for ${ }^{13} \mathrm{C}$ ) and synthetic 3 (synthesized by Wood and co-workers, $600 \mathrm{MHz}$ for ${ }^{1} \mathrm{H}, 151 \mathrm{MHz}$ for ${ }^{13} \mathrm{C}$ ). ${ }^{1}$ This resonance wasn't tabulated explicitly by Wang and co-workers but is present in the ${ }^{13} \mathrm{C}$ spectrum of natural 3. 


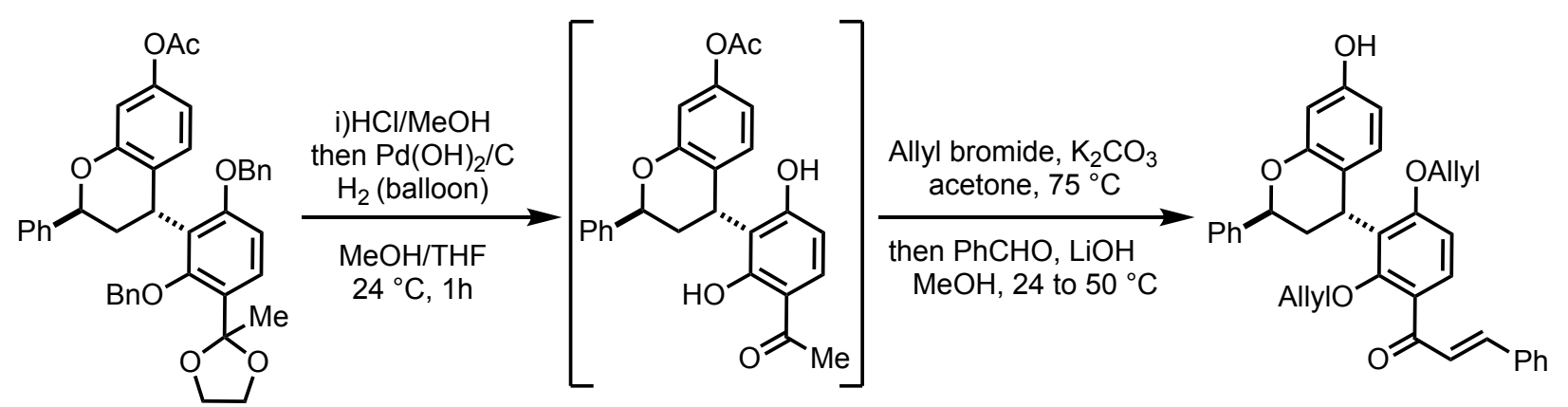

Phenol 12a:

Step 1. To a solution of $10 \mathrm{a}(41.6 \mathrm{mg}, 0.065 \mathrm{mmol})$ in $\mathrm{MeOH}(3.0 \mathrm{~mL})$ was added $10 \%$ aq. $\mathrm{HCl}(20 \mu \mathrm{L})$. The solution stirred at $24^{\circ} \mathrm{C}$ for 15 minutes before being sparged with dry argon for 10 minutes, followed by evacuation under vacuum (ca. 15 torr) for 5 minutes. THF $(3.0 \mathrm{~mL})$ was then added and the solution then sparged with hydrogen gas for 5 minutes before being placed under a static atmosphere of hydrogen gas (Note 1). To the resulting solution was added Pearlman's catalyst (30 mg, Note 2). The resulting suspension was sparged with hydrogen gas for 5 minutes before being placed under an atmosphere of static hydrogen and stirred for 60 minutes. After the reaction was complete, it was rapidly filtered through a plug of Celite, eluting with ethyl acetate (25 $\mathrm{mL}$ ). The resulting solution was concentrated under vacuum to give crude bisphenol 18a. which was used crude in the next step.

Step 2. To a solution of crude bisphenol 18a in acetone $(1.5 \mathrm{~mL})$ in a $10 \mathrm{~mL}$ microwave tube was added allyl bromide $(18.0 \mu \mathrm{L}, 0.21 \mathrm{mmol})$ and potassium carbonate ( $37.7 \mathrm{mg}, 0.22 \mathrm{mmol})$. The tube was then sealed and placed under an atmosphere of dry nitrogen before being heated in a microwave at $75^{\circ} \mathrm{C}$ for 6 hours. The suspension was then cooled to $24^{\circ} \mathrm{C}$ and volatiles removed under vacuum (Note 3). $\mathrm{MeOH}$ (1.5 mL) was then added to the tube and the suspension stirred for 10 minutes before benzaldehyde $(19.0 \mu \mathrm{L}, 0.17 \mathrm{mmol})$ and lithium hydroxide monohydrate $(10 \mathrm{mg}, 0.24 \mathrm{mmol})$ were added. The tube was then heated in a microwave at $50{ }^{\circ} \mathrm{C}$ for 30 minutes. After being cooled to $24^{\circ} \mathrm{C}$, the suspension was quenched with $10 \mathrm{~mL}$ saturated aqueous ammonium chloride. The mixture was then extracted with DCM $(5 \times 5 \mathrm{~mL})$ and the combined organic phases washed with saturated brine $(5 \mathrm{~mL})$, dried over sodium sulfate, filtered, and concentrated. The crude residue was purified by MPLC ( $12 \mathrm{~g}$ gold column, $30 \mathrm{~mL} / \mathrm{min}, 10 \% \rightarrow 60 \%$ Ethyl acetate/hexanes) to give a Phenol 19 a as a yellow oil $(19.9 \mathrm{mg}, 56 \%$ yield over two steps). 19a exhibited broad ${ }^{1} \mathrm{H}$ resonances in $\mathrm{CDCl}_{3}$, while giving relatively sharp ${ }^{1} \mathrm{H}$ resonances in $\mathrm{PhMe}-d_{8}$. Variable temperature NMR in $\mathrm{PhMe}-d_{8}$ suggested the presence of two atropisomers which seemed not to interconvert up at up to $75^{\circ} \mathrm{C}$. For this reason, 19a was carried forward to the next step without rigorous characterization. See Figure S36 for VT NMR spectra of 19a in PhMe-d8.

Note 1: The sparging/vacuum cycle is to degas $\mathrm{HCl}$ from the reaction in order to avoid over reduction of the flavan $\mathrm{C}$ ring $\mathrm{C}-\mathrm{O}$ bond. 
Note 2: We observed a marked effect of the age of Pearlman's catalyst and selectivity for our desired reaction; Fresh bottles of Pearlman's catalyst (from Millipore Sigma) gave mixtures of the desired bisphenol and overreduction, presumably to a trisphenol where the $\mathrm{C}-\mathrm{O}$ bond in the dihydropyran ring had been cleaved. Older bottles (ca. 15-20 years old, Aldrich) were less active and were slower to cleave this more hindered $\mathrm{C}-\mathrm{O}$ bond. However, if the reaction is not filtered through Celite quickly after the removal of both benzyl groups, $\mathrm{C}-\mathrm{O}$ bond cleavage of the flavanoid $\mathrm{C}$-ring begins to occur (as judged by MS analysis of aliquots).

Note 3: It is imperative that the reaction mixture is thoroughly evacuated. If traces of allyl bromide remain during the saponification step, a compound with all three phenols protected as the aryl allyl ethers was obtained in ca. $50 \%$ yield along with the desired compound (ca. $40 \%$ yield). This was accomplished by evacuating the reaction flask for 2 hours under high vacuum (ca. 10 mTorr). 
<smiles>COc1ccc(C(=O)/C=C/c2ccccc2)c(O[Na])c1[C@H]1CC(c2ccccc2)Oc2cc(O)ccc21</smiles>
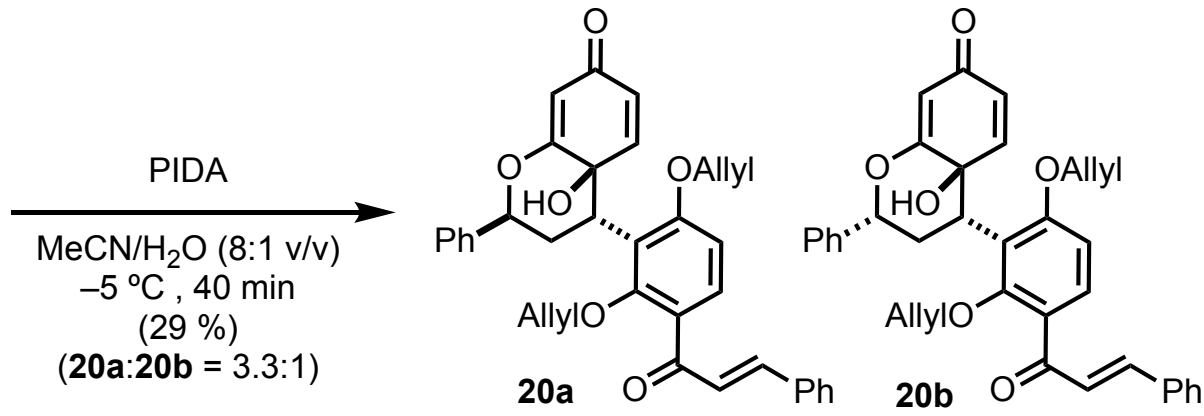

p-quinol 20a:

To a stirred solution of crude $19 \mathrm{a}(8.1 \mathrm{mg}, 0.015 \mathrm{mmol})$ in $8: 1(\mathrm{v} / \mathrm{v})$ solution of acetonitrile/water $(2.0 \mathrm{~mL})$ at $-5^{\circ} \mathrm{C}$ was added PIDA $(8.6 \mathrm{mg}, 0.026 \mathrm{mmol})$ in one portion. The faint-yellow solution was stirred rapidly for 40 minutes to afford a bright orange solution. After completion, as judged by TLC, the solution was concentrated to ca. $0.3 \mathrm{~mL}$ and loaded directly only a $20 \mathrm{~cm} \times 20 \mathrm{~cm}$ TLC plate and developed with EtOAc-hexanes $(1: 1 \mathrm{v} / \mathrm{v})$ to give $20 \mathrm{a} / 20 \mathrm{~b}$ (3.3:1) yellow foam $(2.4 \mathrm{mg}, 29 \%)$

Note 1: This reaction gave variable yields and diastereoselectivities base on scale: 11.2 mg scale: $24 \%$, 20a:20b = 3.6:1), $21.0 \mathrm{mg}$ scale $\left(4^{\circ} \mathrm{C}\right) 31 \%$ yield, $\left.20 \mathrm{a}: 20 \mathrm{~b}=1.2: 1\right)$.

Note 2: The low yield in the phenolic oxidation was mainly attributed to formation of an o-quinone compound (tentative assignment), resulting from attack of water at the ortho-position $\mathrm{C}^{\prime}$ ' of the aromatic ring. Due to the instability of this compound, it wasn't characterized rigorously.

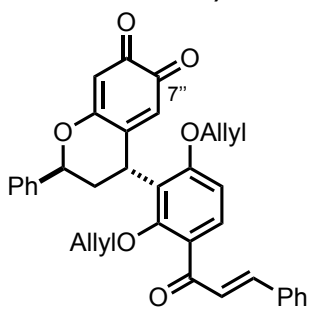

Note 3: 20a partially oxidizes on air during preparative TLC to a compound having 2 fewer hydrogen atoms. Owing to the instability of this oxidized compound, it was not characterized.

${ }^{1} \mathrm{H}$ NMR (600 MHz, Chloroform- $d$ ): $\delta 7.74(\mathrm{~d}, J=15.8 \mathrm{~Hz}, 1 \mathrm{H}), 7.65-7.58(\mathrm{~m}, 3 \mathrm{H}), 7.49$ (d, $J=15.7 \mathrm{~Hz}, 1 \mathrm{H}), 7.45(\mathrm{~d}, J=7.2 \mathrm{~Hz}, 2 \mathrm{H}), 7.44-7.38(\mathrm{~m}, 7 \mathrm{H}), 7.35(\mathrm{~d}, J=7.2 \mathrm{~Hz}$, $1 \mathrm{H}), 6.73(\mathrm{~d}, J=8.8 \mathrm{~Hz}, 1 \mathrm{H}), 6.51(\mathrm{~d}, J=9.9 \mathrm{~Hz}, 1 \mathrm{H}), 6.02-5.88(\mathrm{~m}, 4 \mathrm{H}), 5.74(\mathrm{~d}, J=$ $1.8 \mathrm{~Hz}, 1 \mathrm{H}), 5.52(\mathrm{dd}, J=10.6,3.7 \mathrm{~Hz}, 1 \mathrm{H}), 5.40-5.21(\mathrm{~m}, 5 \mathrm{H}), 5.18(\mathrm{~d}, \mathrm{~J}=10.4 \mathrm{~Hz}$, $1 \mathrm{H}), 4.48-4.45(\mathrm{~m}, 2 \mathrm{H}), 4.44-4.42(\mathrm{~m}, 3 \mathrm{H}), 2.99$ (ddd, J = 14.5, 10.6, 6.9 Hz, $1 \mathrm{H})$, $2.35(\mathrm{~s}, 1 \mathrm{H}), 2.14(\mathrm{dt}, \mathrm{J}=14.5,3.8 \mathrm{~Hz}, 1 \mathrm{H}$ ) (Note: overlap of the aromatic resonances with 16b accounts for larger integration values). $\left.{ }^{13} \mathbf{C ~ N M R ~ ( 1 5 1 ~} \mathbf{M H z}, \mathbf{C D C l}_{3}\right): \delta 191.1$, 187.9, 175.8, 160.3, 158.0, 145.5, 143.8, 140.5, 134.8, 132.5, 131.8, 131.3, 130.5, 128.9, 128.6, 128.5, 128.3, 128.1, 126.1, 125.7, 121.7, 119.1, 119.0, 108.3, 106.5, 79.77, 77.5, 68.3, 66.0, 37.9, 34.4. IR (ATR): $v_{\max }\left(\mathrm{cm}^{-1}\right)=3332(\mathrm{br}), 3030(\mathrm{w}), 2926(\mathrm{w}), 2855(\mathrm{w})$, $1658(\mathrm{~m}), 1592(\mathrm{~s}), 1450(\mathrm{w}), 1337(\mathrm{~m}), 1275(\mathrm{~m}), 1192(\mathrm{~m}), 1084(\mathrm{~m}), 913(\mathrm{~m}), 862(\mathrm{w})$, 
811 (w), $760(\mathrm{~m}), 731(\mathrm{~m}), 700$ (m), 563 (w). HRMS (ESI+) Calcd. for $\mathrm{C}_{36} \mathrm{H}_{32} \mathrm{O}_{6} \mathrm{Na}$. $[\mathrm{M}+\mathrm{Na}]^{+}:$583.2097. Found: 583.2091. TLC (EtOAc-hexanes $\left.=1: 1\right), R_{\mathrm{f}}=0.44$. 
<smiles>C=CCOc1ccc(C(=O)/C=C/c2ccccc2)c(OCC=C)c1[C@H]1CC(c2ccccc2)OC2=CC(=O)C=CC21O</smiles>

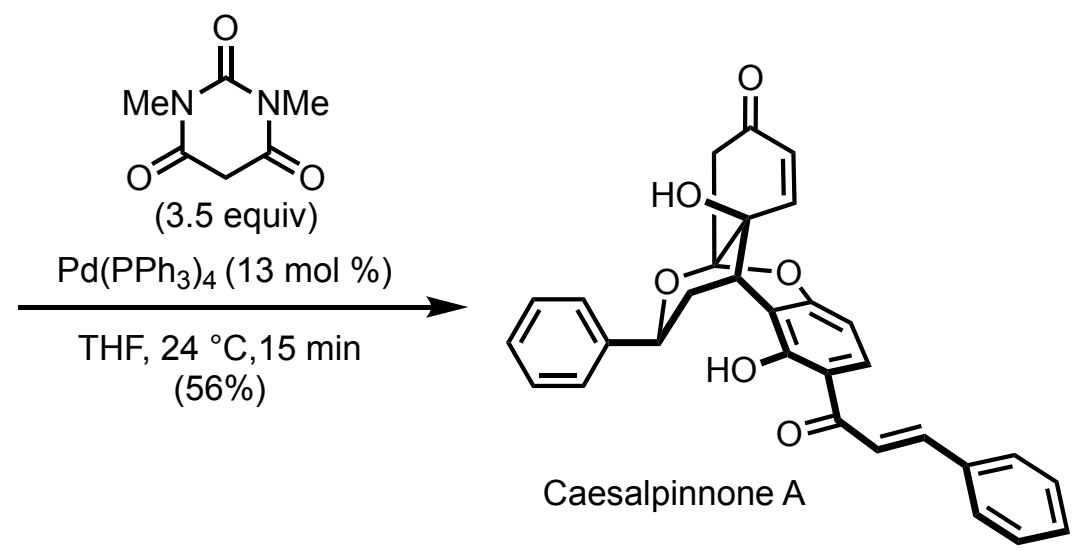

Caesalpinnone A (1):

To a dry 1.5-dram vial containing 20a/20b (3.4 mg, $0.0061 \mathrm{mmol}, 3.3: 1$ mixture), 1,3-dimethyl barbituric acid (3.3 mg, $0.0021 \mathrm{mmol}, 3.5$ equiv), [Pd( $\left.\left(\mathrm{PPh}_{3}\right)_{4}\right](0.9 \mathrm{mg}, 13$ $\mathrm{mol} \%)$, and a magnetic stir-bar was dry THF $(1.5 \mathrm{~mL})$. The bright yellow solution was stirred for 10 minutes before being rapidly filtered through a short plug of silica gel, which was eluted with ethyl acetate $(3 \mathrm{~mL})$. The resulting solution as concentrated under vacuum and purified by reverse-phase preparative TLC (water-acetonitrile $=4: 7$ ). The yellow band was collected affording $1(1.65 \mathrm{mg}, 56 \%)$ as a yellow microcrystalline solid.

${ }^{1} \mathrm{H}$ NMR $\left(600 \mathrm{MHz}\right.$, Acetone- $\left.d_{6}\right): \delta 14.08(\mathrm{~s}, 1 \mathrm{H}), 8.23(\mathrm{~d}, J=8.9 \mathrm{~Hz}, 1 \mathrm{H}), 8.07(\mathrm{~d}, J=$ $15.5 \mathrm{~Hz}, 1 \mathrm{H}), 7.97(\mathrm{~d}, J=15.4 \mathrm{~Hz}, 1 \mathrm{H}), 7.94-7.89(\mathrm{~m}, 2 \mathrm{H}), 7.52-7.50(\mathrm{~m}, 3 \mathrm{H}), 7.44(\mathrm{~d}$, $J=7.1 \mathrm{~Hz}, 2 \mathrm{H}), 7.35(\mathrm{t}, J=7.6 \mathrm{~Hz}, 2 \mathrm{H}), 7.29(\mathrm{t}, J=7.3 \mathrm{~Hz}, 1 \mathrm{H}), 6.84(\mathrm{dd}, J=9.9,1.0 \mathrm{~Hz}$, $1 \mathrm{H}), 6.48(\mathrm{~d}, J=9.0 \mathrm{~Hz}, 1 \mathrm{H}), 5.93(\mathrm{~d}, J=9.9 \mathrm{~Hz}, 1 \mathrm{H}), 5.44(\mathrm{~s}, 1 \mathrm{H}), 4.81$ (dd, $J=12.2,3.5$ $\mathrm{Hz}, 1 \mathrm{H}), 3.74(\mathrm{t}, J=3.5 \mathrm{~Hz}, 1 \mathrm{H}), 3.34(\mathrm{~d}, J=16.8 \mathrm{~Hz}, 1 \mathrm{H}), 2.80$ (partially overlaps with HDO), $2.76(\mathrm{~d}, J=16.7 \mathrm{~Hz}), 1.94(\mathrm{dt}, J=13.3,3.4 \mathrm{~Hz}, 1 \mathrm{H}) .{ }^{13} \mathrm{C}$ NMR (151 MHz, Acetone): $\delta$ 194.6, 192.6, 163.2, 161.6, 148.2, 144.8, 141.3, 134.9, 131.1, 130.8, 130.5, 129.0, 128.9, 128.2, 127.6, 126.2, 120.5, 114.2, 111.4, 106.4, 101.5, 71.5, 64.1, 44.3, 33.7, 32.8. TLC (C18 silica: water-acetonitrile $=4: 7), R_{\mathrm{f}}=0.30$. IR (ATR): $v_{\max }\left(\mathrm{cm}^{-1}\right)=3381(\mathrm{br})$, $2926(\mathrm{~m}), 2853(\mathrm{w}), 1667(\mathrm{~s}), 1634(\mathrm{~s}), 1594(\mathrm{~s}), 1487(\mathrm{~m}), 1450(\mathrm{~m}), 1421(\mathrm{~m}), 1359(\mathrm{~s})$, 1278 (s), 1223 (s), 1178 (w), 1102 (s), 1009 (s), 994 (s), 885 (w), 854 (w), 788 (m), 699 (m), 570 (w). HRMS (ESI+) Calcd. for $\mathrm{C}_{30} \mathrm{H}_{25} \mathrm{O}_{5}[\mathrm{M}+\mathrm{H}]$ ] 481.1651, found: 481.1644. TLC $\left(C_{18}\right.$ silica: water-acetonitrile $\left.=4: 7\right), R_{\mathrm{f}}=0.53$. 


\begin{tabular}{|c|c|c|c|c|c|c|}
\hline \multicolumn{7}{|c|}{${ }^{13} \mathrm{C}$ NMR Chemical Shifts } \\
\hline position & $\begin{array}{c}\text { Natural } \\
\text { (+)-Caesalpinnone A (1) }\end{array}$ & $\begin{array}{c}\text { Synthetic } \\
\text { ( } \pm \text {-Caesalpinnone A (1) }\end{array}$ & $\Delta \mathrm{ppm}$ & $\begin{array}{c}\text { Natural } \\
\text { (+)-Caesalpinnone A (1) }\end{array}$ & $\begin{array}{l}\text { Synthetic } \\
\text { ( } \pm \text {-Caesalpinnone A (1) }\end{array}$ & $\Delta \mathrm{ppm}$ \\
\hline 1 & - & - & $\mathrm{n} / \mathrm{a}$ & 134.9 & 134.9 & - \\
\hline 2,6 & 7.90 & 7.91 & 0.01 & 129.0 & 129.0 & - \\
\hline 3,5 & 7.50 & 7.50 & - & 128.9 & 128.9 & - \\
\hline 4 & 7.50 & 7.50 & - & 130.8 & 130.8 & - \\
\hline a & $7.97(\mathrm{~d}, 15.0)$ & $7.97(d, 15.4)$ & - & 144.7 & 144.7 & - \\
\hline$b$ & $8.06(d, 15.0)$ & $8.07(d, 15.5)$ & 0.01 & 120.6 & 120.6 & - \\
\hline$y$ & - & - & $\mathrm{n} / \mathrm{a}$ & 192.5 & 192.6 & 0.1 \\
\hline 1 ' & - & - & $\mathrm{n} / \mathrm{a}$ & 114.2 & 114.2 & - \\
\hline 2 & - & - & $n / a$ & 163.2 & 163.2 & - \\
\hline 3 & - & - & $n / a$ & 111.4 & 111.4 & - \\
\hline 4' & - & - & $\mathrm{n} / \mathrm{a}$ & 161.6 & 161.6 & - \\
\hline 5 & $6.48(d, 9.0)$ & $6.48(d, 9.0)$ & - & 106.5 & 106.5 & - \\
\hline 6 ' & $8.22(d, 9.0)$ & $8.23(\mathrm{~d}, 8.9)$ & 0.01 & 131.2 & 131.2 & - \\
\hline 2" & $4.82(\mathrm{dd}, 3.5,12.2)$ & $4.81(\mathrm{dd}, 3.5,12.2)$ & 0.01 & 71.6 & 71.6 & - \\
\hline $3 "$ & $2.81(\mathrm{~m})$ & $2.80^{1}$ & 0.01 & 33.6 & 33.7 & 0.1 \\
\hline & $1.91(\mathrm{td}, 3.3,13.3)$ & $1.94(\mathrm{td}, 3.4,13.3)$ & 0.03 & & & \\
\hline $4 "$ & $3.74(t, 3.5)$ & $3.74(\mathrm{t}, 3.5)$ & - & 32.9 & 32.9 & - \\
\hline $5 "$ & $6.84(\mathrm{~d}, 10.0)$ & $6.84(d, 9.9)$ & - & 148.2 & 148.2 & - \\
\hline $6 "$ & $5.93(d, 9.8)$ & $5.93(d, 9.9)$ & - & 130.5 & 130.5 & - \\
\hline 7" & - & - & - & 194.6 & 194.6 & - \\
\hline 8 & $2.76(\mathrm{~m})$ & $2.75(\mathrm{~m})$ & 0.01 & 44.4 & 44.4 & - \\
\hline & $3.36(d, 16.0)$ & $3.34(d, 16.8)$ & 0.02 & & & \\
\hline $9 "$ & - & - & $\mathrm{n} / \mathrm{a}$ & 101.5 & 101.5 & - \\
\hline $10 "$ & - & - & $n / a$ & 64.1 & 64.1 & - \\
\hline $1 " \prime$ & - & - & $n / a$ & 141.3 & 141.3 & - \\
\hline 2"',6"' & $7.43(\mathrm{~d}, 8.5)$ & $7.44(\mathrm{~d}, 7.1)$ & 0.01 & 126.2 & 126.2 & - \\
\hline 3"',5”' & $7.35(t, 7.5)$ & $7.35(\mathrm{t}, 7.6)$ & - & 128.2 & 128.2 & - \\
\hline 4"' & $7.28(t, 7.1)$ & $7.29(\mathrm{t}, 7.3)$ & 0.01 & 127.6 & 127.6 & - \\
\hline 2'-OH & 14.07 (s) & 14.08 (s) & 0.01 & - & - & $\mathrm{n} / \mathrm{a}$ \\
\hline 10"-OH & $5.53(\mathrm{~s})$ & $5.44(\mathrm{~s})$ & $0.09^{2}$ & - & - & $\mathrm{n} / \mathrm{a}$ \\
\hline
\end{tabular}

Table S3. Comparison of ${ }^{1} \mathrm{H}$ chemical shifts and multiplicities between natural 1 (isolated by Wang and co-workers, ${ }^{56} 500 \mathrm{MHz}$ for ${ }^{1} \mathrm{H}, 125 \mathrm{MHz}$ for ${ }^{13} \mathrm{C}$ ) and synthetic 1 (synthesized by Wood and co-workers, $600 \mathrm{MHz}$ for ${ }^{1} \mathrm{H}, 151 \mathrm{MHz}$ for ${ }^{13} \mathrm{C}$ ). ${ }^{1}$ This resonance is partially obscured with $\mathrm{HDO}(2.81 \mathrm{ppm})$ in acetone- $d_{6} .{ }^{2}$ We attribute the marginal chemical shift difference in the 10 "- $\mathrm{O}-\mathrm{H}$ resonance between the natural and synthetic sample to concentration differences. 


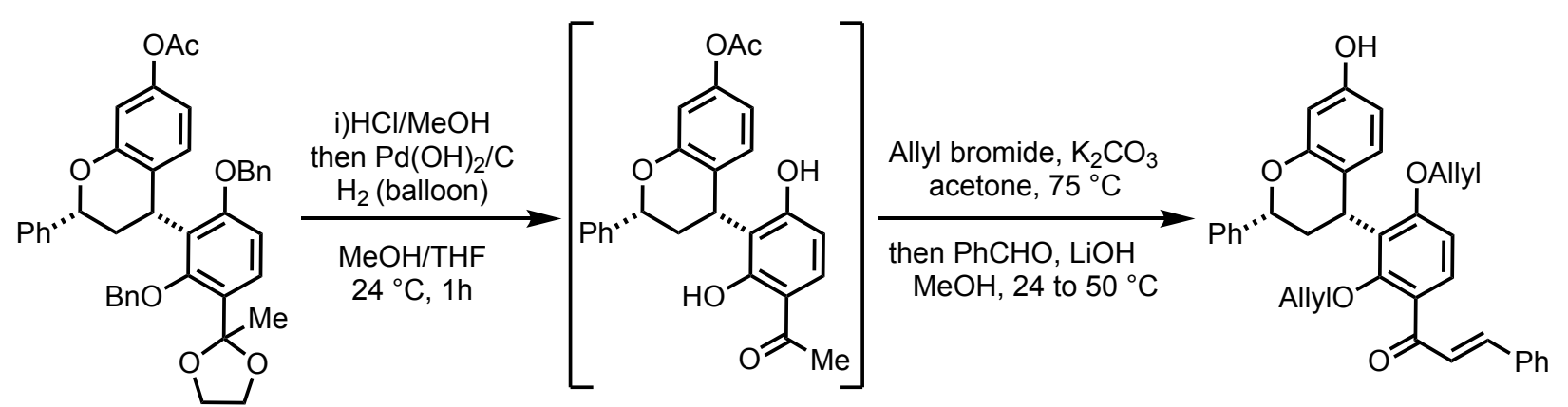

Phenol 19b:

Step 1.To a solution of $10 \mathrm{~b}(59.2 \mathrm{mg}, 0.0922 \mathrm{mmol})$ in $\mathrm{MeOH}(2.5 \mathrm{~mL})$ was added $10 \%$ aq. $\mathrm{HCl}(20 \mathrm{uL})$. The solution stirred at $24{ }^{\circ} \mathrm{C}$ for 15 minutes before being sparged with dry argon for 10 minutes, followed by evacuation under vacuum (ca. 15 torr) for 5 minutes, before being placed under a static atmosphere of hydrogen gas (Note 1). To the resulting solution was added THF $(2.5 \mathrm{~mL})$, followed by Pearlman's catalyst ( $35 \mathrm{mg}$, Note 2). The resulting suspension was sparged with hydrogen gas for 5 minutes before being placed under an atmosphere of static hydrogen and stirred for 40 minutes. After the reaction was complete, it was rapidly filtered through a plug of Celite, eluting with ethyl acetate $(15 \mathrm{~mL})$. The resulting solution was concentrated under vacuum to give crude bisphenol 18b (46.2 mg), which was used crude in the next step.

Step 2. To a solution of crude bisphenol $18 \mathrm{~b}(46.2 \mathrm{mg})$ in acetone $(1.5 \mathrm{~mL})$ in a 10 $\mathrm{mL}$ microwave tube was added allyl bromide ( $24.6 \mu \mathrm{L}, 0.279 \mathrm{mmol}, 3$ equiv) and potassium carbonate (43.6 mg, 3.4 equiv). The tube was then sealed and placed under an atmosphere of dry nitrogen before being heated in a microwave at $75^{\circ} \mathrm{C}$ for 4 hours. The suspension was then cooled to $24^{\circ} \mathrm{C}$ and volatiles removed under vacuum (Note 3). $\mathrm{MeOH}(1.5 \mathrm{~mL})$ was then added to the tube and the suspension stirred for 10 minutes before benzaldehyde $(22.8 \mu \mathrm{L}, 0.204 \mathrm{mmol})$ and lithium hydroxide monohydrate $(10 \mathrm{mg})$ were added. The tube was then heated in a microwave at $50{ }^{\circ} \mathrm{C}$ for 30 minutes. After being cooled to $24{ }^{\circ} \mathrm{C}$, the suspension was quenched with $5 \mathrm{~mL}$ saturated aqueous ammonium chloride. The mixture was extracted with DCM $(5 \times 5 \mathrm{~mL})$ and the combined organic phases washed with saturated brine $(5 \mathrm{~mL})$, dried over sodium sulfate, filtered, and concentrated. The crude residue was purified by MPLC (12 g gold column, 30 $\mathrm{mL} / \mathrm{min}, 10 \% \rightarrow 60 \%$ Ethyl acetate/hexanes to give $19 \mathrm{~b}$ as a yellow foam $(29.8 \mathrm{mg}, 59 \%)$ as an inseparable 3:1 mixture of atropisomers.

Note 1: The sparging/vacuum cycle is to degas $\mathrm{HCl}$ from the reaction in order to avoid over reduction of the flavanoid- $\mathrm{C}$ ring $\mathrm{C}-\mathrm{O}$ bond.

Note 2: We observed a marked effect of the age of Pearlman's catalyst and selectivity for our desired reaction; Fresh bottles of Pearlman's catalyst (from Millipore Sigma) gave mixtures of the desired bisphenol and overreduction, presumably to a trisphenol where the $\mathrm{C}-\mathrm{O}$ bond in the dihydropyran ring had been cleaved. Older bottles (ca. 15-20 years old, Aldrich) were less active and were slower to cleave this more hindered $\mathrm{C}-\mathrm{O}$ bond. 
However, if the reaction is not filtered through Celite quickly after the removal of both benzyl groups, $\mathrm{C}-\mathrm{O}$ bond cleavage of the flavanoid C-ring begins to occur (as judged by MS analysis of aliquots).

Note 3: It is imperative that the reaction mixture is thoroughly evacuated. If traces of allyl bromide remain during the saponification step, a compound with all three phenols protected as the aryl allyl ethers was obtained in ca. $50 \%$ yield along with the desired compound (ca. $40 \%$ yield). This was accomplished by evacuating the reaction flask for 2 hours under high vacuum (ca. 10 mTorr).

${ }^{1} \mathrm{H}$ NMR (600 MHz, Chloroform- $d, 3: 1$ mixture of atropisomers, all resonances) $\delta$ $7.78(\mathrm{~d}, J=15.8 \mathrm{~Hz}, 1 \mathrm{H}), 7.70(\mathrm{~d}, J=15.9 \mathrm{~Hz}, 0 \mathrm{H}), 7.67(\mathrm{~d}, J=8.6 \mathrm{~Hz}, 1 \mathrm{H}), 7.65-7.58$ $(\mathrm{m}, 3 \mathrm{H}), 7.56-7.51(\mathrm{~m}, 1 \mathrm{H}), 7.48(\mathrm{~d}, J=7.6 \mathrm{~Hz}, 2 \mathrm{H}), 7.45-7.30(\mathrm{~m}, 10 \mathrm{H}), 7.26(\mathrm{~s}, 1 \mathrm{H})$, $7.15(\mathrm{~d}, J=7.4 \mathrm{~Hz}, 1 \mathrm{H}), 6.80(\mathrm{~d}, J=8.7 \mathrm{~Hz}, 1 \mathrm{H}), 6.71(\mathrm{~d}, J=8.6 \mathrm{~Hz}, 1 \mathrm{H}), 6.59(\mathrm{~d}, J=8.4$ $\mathrm{Hz}, 1 \mathrm{H}), 6.56(\mathrm{~d}, J=8.4 \mathrm{~Hz}, 1 \mathrm{H}), 6.46(\mathrm{~d}, J=2.5 \mathrm{~Hz}, 1 \mathrm{H}), 6.43(\mathrm{~d}, J=2.6 \mathrm{~Hz}, 0 \mathrm{H}), 6.28$ (dd, $J=8.4,2.5 \mathrm{~Hz}, 1 \mathrm{H}$ ), 6.10 (ddt, $J=16.3,10.5,5.3 \mathrm{~Hz}, 1 \mathrm{H}$ ), 6.01 (ddt, $J=16.4,10.9$, $5.8 \mathrm{~Hz}, 1 \mathrm{H}), 5.62(\mathrm{ddq}, J=14.5,9.2,4.6 \mathrm{~Hz}, 1 \mathrm{H}), 5.52$ ? $5.42(\mathrm{~m}, 1 \mathrm{H}), 5.37-5.29(\mathrm{~m}$, $1 \mathrm{H}), 5.22-5.08(\mathrm{~m}, 6 \mathrm{H}), 4.98(\mathrm{ddt}, J=25.0,18.5,10.1 \mathrm{~Hz}, 3 \mathrm{H}), 4.75-4.63(\mathrm{~m}, 1 \mathrm{H})$, $4.47-4.32(\mathrm{~m}, 4 \mathrm{H}), 4.26-4.18(\mathrm{~m}, 1 \mathrm{H}), 3.93(\mathrm{dd}, J=11.6,5.9 \mathrm{~Hz}, 1 \mathrm{H}), 3.36-3.26(\mathrm{~m}$, $1 \mathrm{H}), 3.05(\mathrm{q}, J=12.0 \mathrm{~Hz}, 1 \mathrm{H}), 2.78(\mathrm{q}, J=12.3 \mathrm{~Hz}, 1 \mathrm{H}), 2.25-2.18(\mathrm{~m}, 1 \mathrm{H}), 2.14$ (ddd, $J=13.3,6.1,1.9 \mathrm{~Hz}, 1 \mathrm{H}) .{ }^{13} \mathrm{C} \mathrm{NMR}\left(151 \mathrm{MHz}, \mathrm{CDCl}_{3}, 3: 1\right.$ mixture of atropisomers, all resonances) $\delta 191.8,191.6,160.9,160.6,158.6,158.1,156.4,156.0,154.7,154.6$, 143.8, 143.4, 141.6, 141.4, 135.0, 134.8, 132.9, 132.9, 132.7, 132.1, 130.7, 130.5, 130.3, $130.3,128.9,128.8,128.8,128.7,128.6,128.5,128.5,128.5,128.5,128.4,128.2,128.0$, $127.9,127.8,126.6,126.4,126.2$, 126.1, 126.0, 125.55, 119.0, 118.9, 118.3, 118.0, $117.5,117.0,109.1,107.9,107.9,107.6,103.8,103.4,78.9,78.7,77.8,76.0,69.6,69.0$, 35.3, 35.1, 32.9, 31.9. HRMS (ESI+) Calcd. for $\mathrm{C}_{36} \mathrm{H}_{32} \mathrm{O}_{5} \mathrm{Na}$. [M+Na] $]^{+}$: 567.2147. Found: 567.2143. TLC (EtOAc-hexanes $=1: 3), R_{\mathrm{f}}=0.31$. 

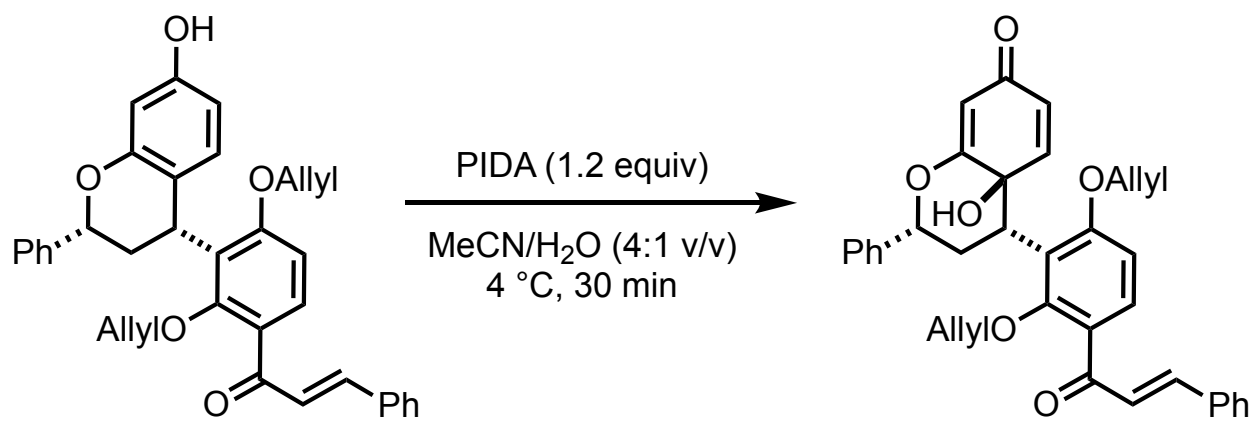

(ca. $85 \%$ purity)

\section{p-quinol 20b:}

To a stirred solution of $19 \mathrm{~b}(29.5 \mathrm{mg}, 0.054 \mathrm{mmol})$ in a $4: 1(\mathrm{v} / \mathrm{v})$ solution of acetonitrile/water at $4{ }^{\circ} \mathrm{C}$ was added PIDA (20.8 mg, $0.058 \mathrm{mmol}, 1.1$ equiv) in one portion. The faint-yellow solution was stirred for 30 minutes to afford a bright orange solution. After completion, as judged by TLC, silica gel (ca. $1 \mathrm{~g}$ ) was added and the suspension concentrated to dryness under vacuum. The solid was then chromatographed directly via MPLC (4 g gold, $12 \mathrm{~mL} / \mathrm{min}, 12 \mathrm{~min}, 10 \% \rightarrow 50 \%$ ethyl acetate/hexanes, 12 minutes) to give $20 \mathrm{~b}$ as a yellow foam (13.9 $\mathrm{mg}, 46 \%$ ) (Note 1).

Note 1: Attempts at obtaining analytically-pure samples of this mixture were met with significant loss of material. However, the chromatographed material corresponding to $\mathbf{2 0 b}$ was reasonably pure by ${ }^{1} \mathrm{H}$ and ${ }^{13} \mathrm{C}$ NMR (ca. $85 \%$ purity) and characterized in this state.

${ }^{1} \mathrm{H}$ NMR (500 MHz, Chloroform- $d$ ) ${ }^{1} \mathrm{H}$ NMR $(500 \mathrm{MHz}$, Chloroform- $d$ ) $\delta 7.73$ (d, J=15.8 $\mathrm{Hz}, 1 \mathrm{H}), 7.65-7.57(\mathrm{~m}, 3 \mathrm{H}), 7.52(\mathrm{~d}, J=15.8 \mathrm{~Hz}, 1 \mathrm{H}), 7.49-7.29(\mathrm{~m}, 9 \mathrm{H}), 6.64(\mathrm{~d}, J=$ $8.7 \mathrm{~Hz}, 1 \mathrm{H}), 6.40(\mathrm{~d}, J=9.9 \mathrm{~Hz}, 1 \mathrm{H}), 6.06$ (ddt, $J=16.5,11.0,6.0 \mathrm{~Hz}, 1 \mathrm{H}), 6.01-5.86$ $(\mathrm{m}, 2 \mathrm{H}), 5.82(\mathrm{dd}, J=9.9,1.7 \mathrm{~Hz}, 1 \mathrm{H}), 5.67(\mathrm{~d}, J=1.7 \mathrm{~Hz}, 1 \mathrm{H}), 5.39-5.20(\mathrm{~m}, 4 \mathrm{H}), 4.50$ ? $4.27(\mathrm{~m}, 5 \mathrm{H}), 2.82(\mathrm{~s}, 1 \mathrm{H}), 2.48-2.32(\mathrm{~m}, 1 \mathrm{H}), 2.31-2.16(\mathrm{~m}, 1 \mathrm{H}) .{ }^{13} \mathrm{C}$ NMR $(151$ MHz, $\left.\mathrm{CDCl}_{3}\right): \delta$ 191.19, 187.85, 174.69, 160.49, 157.40, 144.05, 143.72, 140.08, 134.87, $132.71,132.09,131.48,130.47,128.93,128.66,128.59,128.56,128.51,126.72,126.35$, $125.89,121.63,119.42,118.98,108.04,104.32,77.79,77.57,69.06,68.52,39.20,34.90$. IR (ATR): $v_{\max }\left(\mathrm{cm}^{-1}\right) 3310(\mathrm{br}), 3065(\mathrm{w}), 3029(\mathrm{w}), 2927(\mathrm{~m}), 2864(\mathrm{w}), 2352(\mathrm{w}), 2040$ (w), $1660(\mathrm{~s}), 1593(\mathrm{~s}), 1450(\mathrm{~m}), 1415(\mathrm{~m}), 1337(\mathrm{~m}), 1274(\mathrm{~m}), 1196(\mathrm{~m}), 1091(\mathrm{~s}), 990$ $\left(\mathrm{m}, 859(\mathrm{~m}), 810(\mathrm{w}), 761(\mathrm{~m}), 700(\mathrm{~m})\right.$. HRMS (ESI $\left.{ }^{+}\right)$Calcd. for $\mathrm{C}_{36} \mathrm{H}_{32} \mathrm{O} 6 \mathrm{Na}$. [M+Na] ${ }^{+}$: 583.2097. Found: 583.2089. TLC (EtOAc-hexanes $=1: 3), R_{\mathrm{f}}=0.13$ 
<smiles>COc1ccc(C(=O)/C=C/c2ccccc2)c(OC(C)C)c1[C@H]1C[C@H](c2ccccc2)OC2=CC(=O)C=CC21O</smiles>
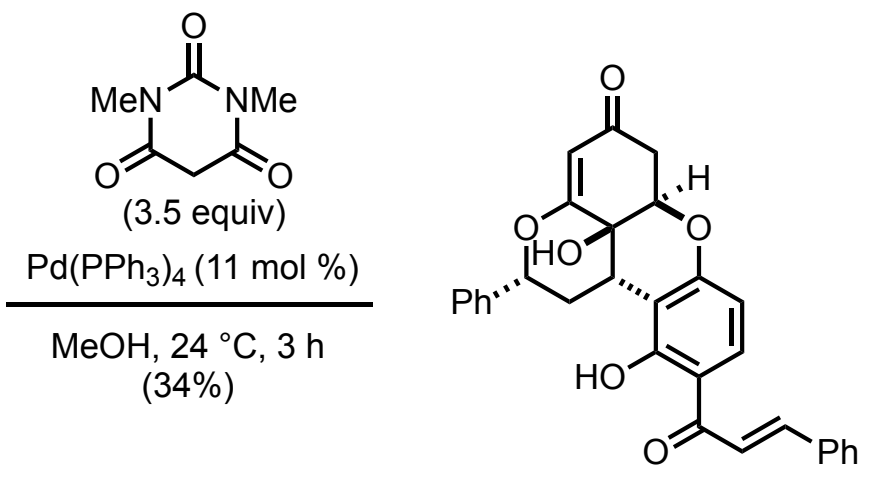

Tricycle 21:

To a dry 1.5-dram vial containing 1,3-dimethyl barbituric acid $(5.4 \mathrm{mg}, 0.033 \mathrm{mmol}, 3.5$ equiv), $\left[\mathrm{Pd}\left(\mathrm{PPh}_{3}\right)_{4}\right](1.2 \mathrm{mg}, 11 \mathrm{~mol} \%)$, and a magnetic stir-bar was added $20 \mathrm{~b}(5.0 \mathrm{mg}$, $0.0092 \mathrm{mmol}, 1$ equiv) as a solution in dry $\mathrm{MeOH}(2.0 \mathrm{~mL})$. The bright yellow solution was stirred for 3 hours before being rapidly filtered through a short plug of silica gel, which was eluted with ethyl acetate $(5 \mathrm{~mL})$. The resulting solution as concentrated under vacuum and purified by reverse-phase preparative TLC (water-acetonitrile $=3: 7$ ). The yellow-orange band was collected affording tricycle $21(1.4 \mathrm{mg}, 34 \%)$ as a yellow foam. The relative stereochemistry was assigned by the combination of NOESY analysis and MM2 optimized geometry calculations of the optimized geometries of 21 and its possible diastereomer 21' (Table S5), and is also corroborated by literature examples of oxaMichael addition reactions into $p$-quinol-derived scaffolds described by Pettus. ${ }^{S 7}$

${ }^{1} \mathrm{H}$ NMR (600 MHz, Acetone- $\left.d_{6}\right): \delta 14.21(\mathrm{~s}, 1 \mathrm{H}), 8.17(\mathrm{~d}, J=8.9 \mathrm{~Hz}, 1 \mathrm{H}), 8.03(\mathrm{~d}, J=$ $15.4 \mathrm{~Hz}, 1 \mathrm{H}), 7.96-7.86(\mathrm{~m}, 3 \mathrm{H}), 7.56-7.47(\mathrm{~m}, 6 \mathrm{H}), 7.41(\mathrm{t}, J=7.6 \mathrm{~Hz}, 2 \mathrm{H}), 7.39-$ $7.32(\mathrm{~m}, 2 \mathrm{H}), 6.56(\mathrm{~d}, J=9.0 \mathrm{~Hz}, 1 \mathrm{H}), 5.79(\mathrm{~d}, J=11.5 \mathrm{~Hz}, 1 \mathrm{H}), 5.73(\mathrm{~s}, 1 \mathrm{H}), 5.54(\mathrm{~s}$, $1 \mathrm{H}), 4.39(\mathrm{t}, J=2.9 \mathrm{~Hz}, 1 \mathrm{H}), 3.87-3.79(\mathrm{~m}, 1 \mathrm{H}), 3.28-3.20(\mathrm{~m}, 2 \mathrm{H}), 2.69(\mathrm{dd}, J=16.9$, $3.0 \mathrm{~Hz}, 1 \mathrm{H}), 1.65$ (ddd, $J=14.6,11.8,10.7 \mathrm{~Hz}, 1 \mathrm{H}) .{ }^{13} \mathrm{C}$ NMR (151 MHz, Acetone- $\left.d_{6}\right): \delta$ 193.1, 192.7, 169.6, 164.1, 144.8, 140.2, 134.9, 130.8, 130.0, 129.7, 129.0, 128.5, 128.2, 126.2, 120.6, 114.9, 114.6, 108.5, 106.9, 78.01, 76.43, 66.6, 37.9, 37.8, 37.3. TLC (C 18 silica: water-acetonitrile $=3: 7), R_{f}=0.34$. HRMS $\left(\mathbf{E S I}^{+}\right)$Calcd. for $\mathrm{C}_{30} \mathrm{H}_{25} \mathrm{O}_{5}[\mathrm{M}+\mathrm{H}]$ : 481.1651, found: 481.1646 


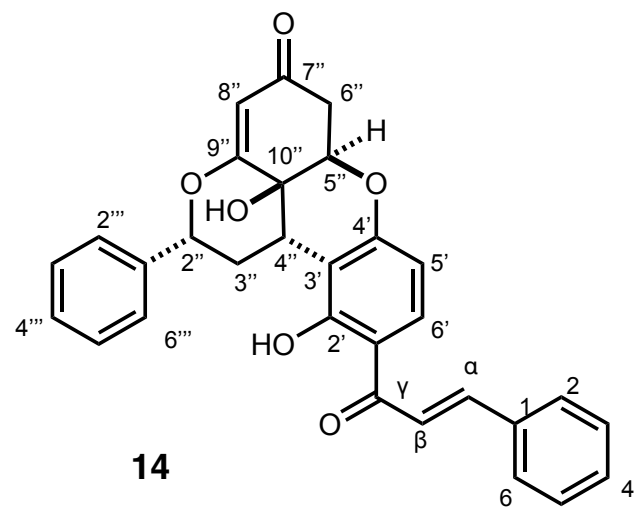

\begin{tabular}{|c|c|c|}
\hline position & ${ }^{1} \mathrm{H}$ NMR Chemical Shifts & ${ }^{13} \mathrm{C}$ NMR Chemical Shifts \\
\hline 1 & - & 134.9 \\
\hline 2,6 & 7.96 - 7.86 (overlap) & 128.5 \\
\hline 3,5 & $7.56-7.47$ & 129.0 \\
\hline 4 & $7.56-7.47$ & 130.8 \\
\hline a & $7.96-7.86$ (overlap) & 144.8 \\
\hline b & $8.03(\mathrm{~d}, 15.4)$ & 120.6 \\
\hline$y$ & - & 192.7 \\
\hline $1^{\prime}$ & - & 114.6 \\
\hline $2^{\prime}$ & - & 164.1 \\
\hline $3^{\prime}$ & - & 114.9 \\
\hline $4^{\prime}$ & - & 161.6 \\
\hline $5^{\prime}$ & $6.56(d, 9.0)$ & 108.5 \\
\hline $6^{\prime}$ & $8.17(\mathrm{~d}, 8.9)$ & 129.7 \\
\hline $2 "$ & $5.79(\mathrm{~d}, 11.5)$ & 76.4 \\
\hline \multirow[t]{2}{*}{$3 "$} & $3.28-3.20$ (overlap) & \\
\hline & $1.65(\mathrm{ddd}, 14.6,11.8,10.7)$ & 37.3 \\
\hline $4 "$ & $3.87-3.79(\mathrm{~m})$ & 37.8 \\
\hline $5 "$ & $4.39(t, 2.9)$ & 78.0 \\
\hline \multirow[t]{2}{*}{$6 "$} & $3.28-3.20$ (overlap) & \\
\hline & $2.69(\mathrm{dd}, 16.9,3.0)$ & 37.9 \\
\hline 7" & - & 193.1 \\
\hline $8 "$ & $5.54(\mathrm{~s})$ & 106.9 \\
\hline 9" & - & 169.6 \\
\hline $10 "$ & - & 66.6 \\
\hline $1 " \prime$ & - & 140.2 \\
\hline 2"',6"' & $7.41(t, 7.6)$ & 128.5 \\
\hline 3"',5”"' & $7.39-7.32$ & 128.2 \\
\hline 4"' & 7.59 - 7.47 (overlap) & 126.2 \\
\hline 2'-OH & $14.21(\mathrm{~s})$ & - \\
\hline 10"-OH & $5.73(\mathrm{~s})$ & - \\
\hline
\end{tabular}

Table S4. ${ }^{1} \mathrm{H}$ and ${ }^{13} \mathrm{C}$ NMR chemical shifts of tricycle 14 based on HSQC and HMBC analysis. 
Figure S3. NOESY analysis of tricycle 21.

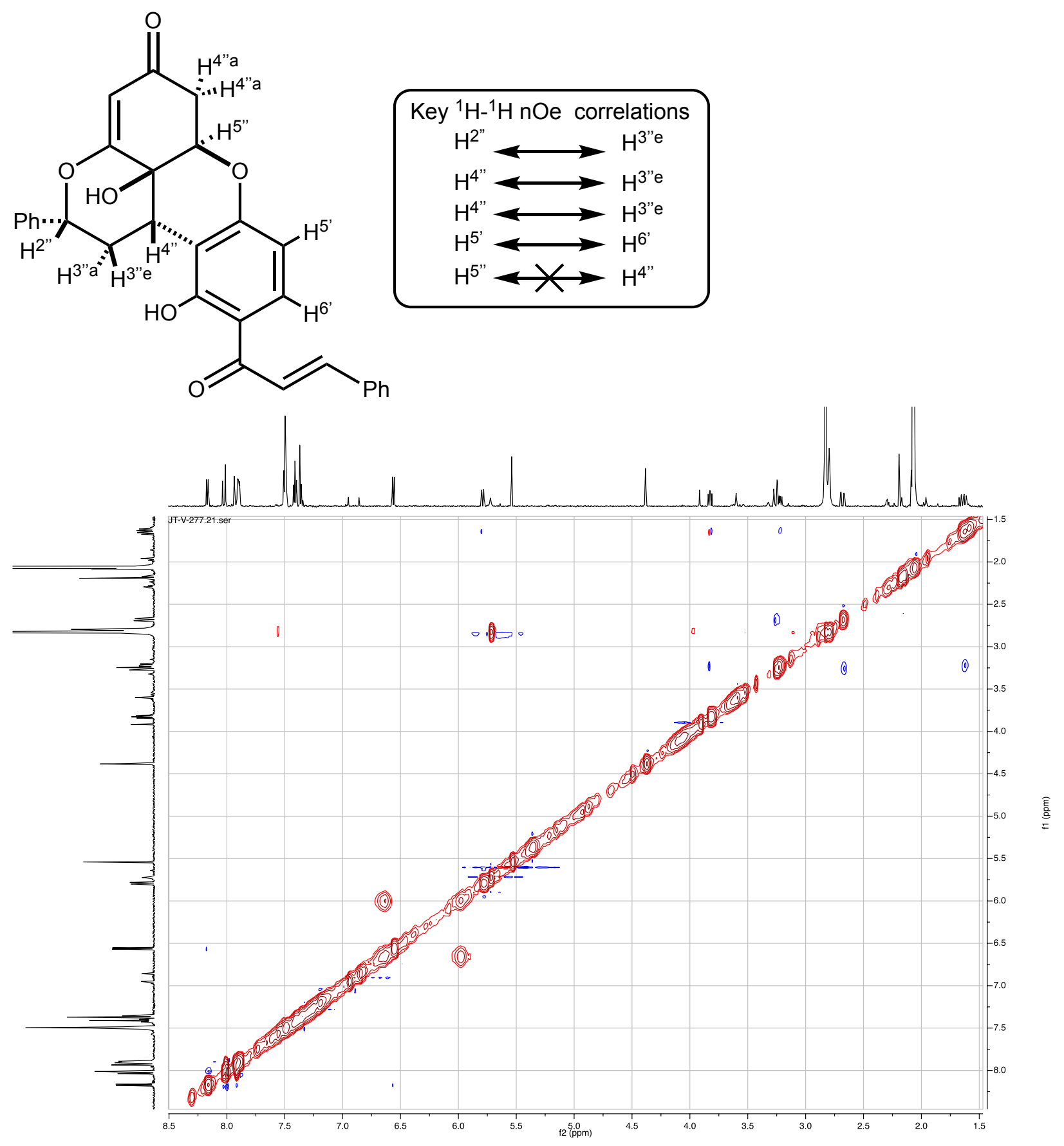


Figure S4. COSY analysis of tricycle 21.
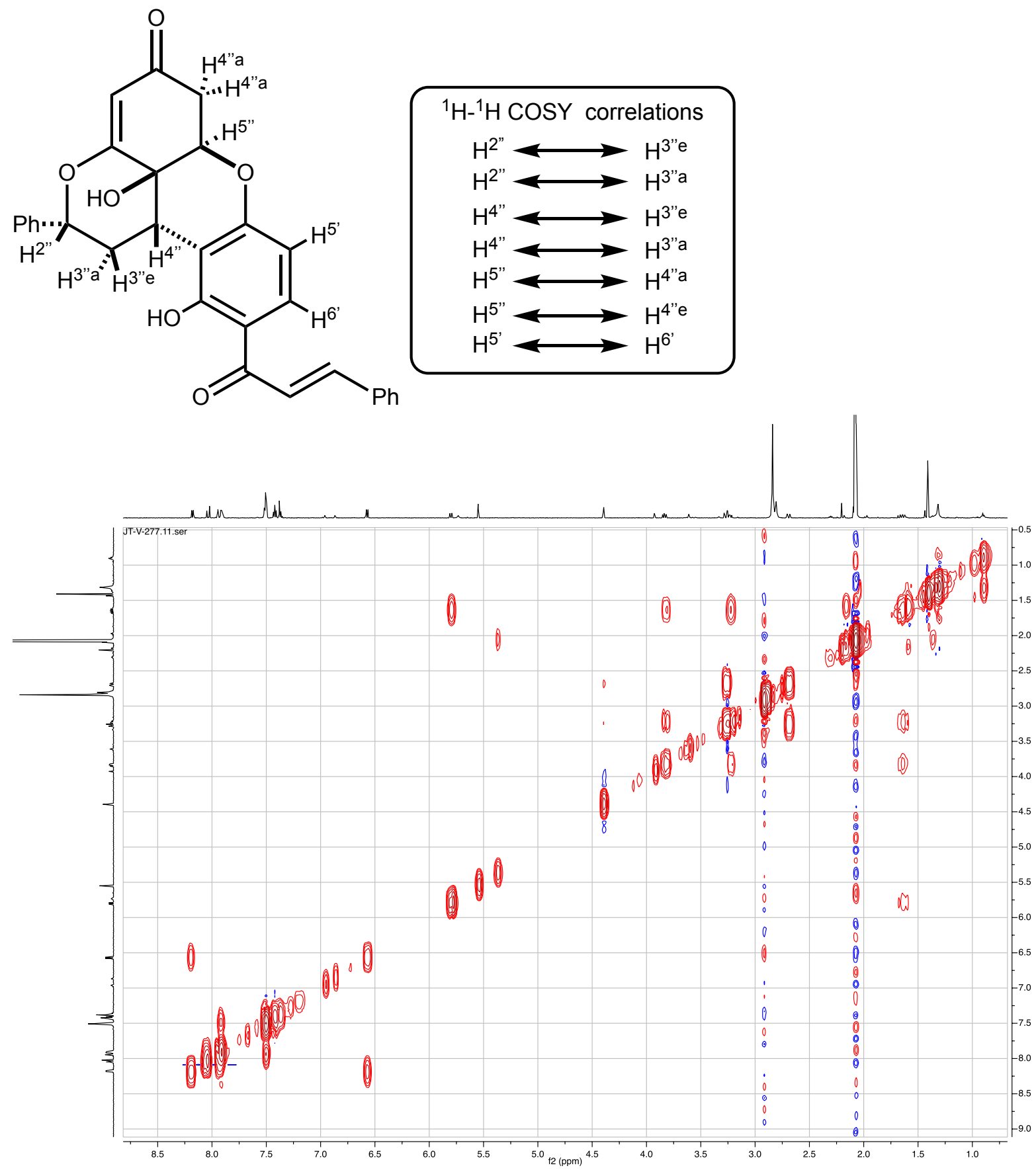
Table S5: Relative energies of tricycles 21, 21', and epi-1.

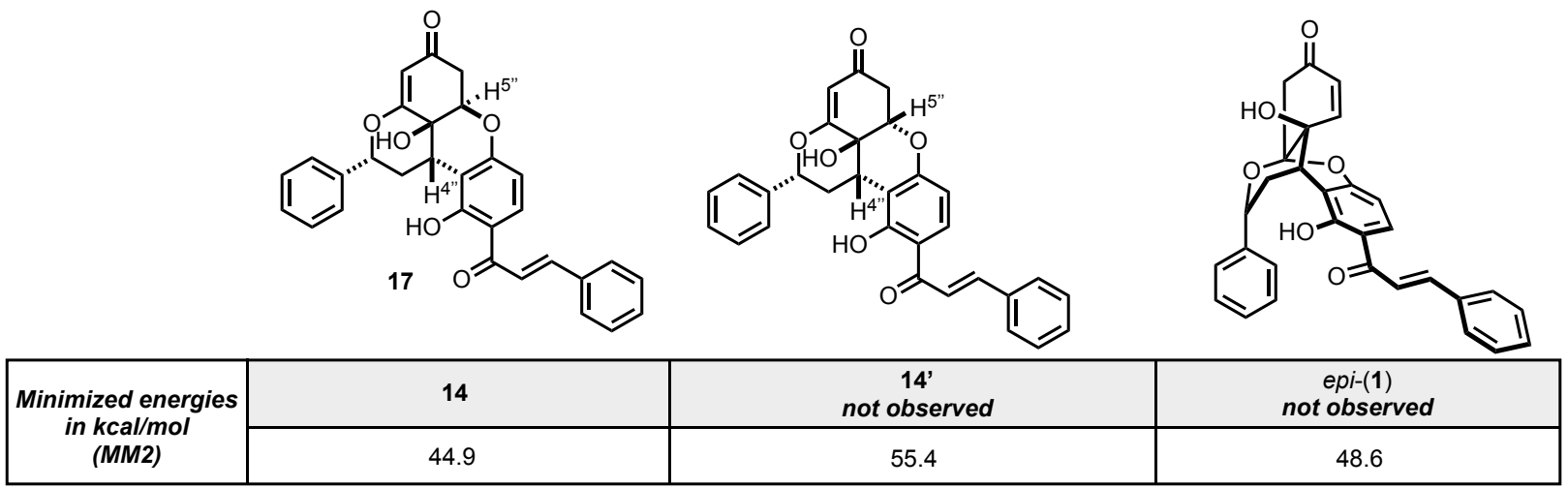

MM2 calculations were performed on the compounds above and showed that tricycle 21, resulting from phenol addition from the $\alpha$-face of the corresponding $p$-quinol $\mathbf{2 0 b}$, is ca. $10.5 \mathrm{kcal} / \mathrm{mol}$ more stable than it's corresponding diastereomer $\mathbf{2 1}$ ', which would result from phenol addition from the $\beta$-face of the corresponding $p$-quinol 20b. Likewise, we also calculated that $\mathbf{2 1}$ is ca. $3.7 \mathrm{kcal} / \mathrm{mol}$ more stable than epi-(1), which we were originally expecting from the deallylation/cyclization reaction of $\mathbf{2 0 b}$. 
Table S6. Examination of other protected substrates for HAT Hydrogenation<smiles>[R]Oc1ccc2c(c1)O[C@H](c1ccccc1)C=C2c1c(OCc2ccccc2)ccc(C2(C)OCCO2)c1OCc1ccccc1</smiles>

$\mathrm{Mn}(\mathrm{dpm})_{3}(10 \mathrm{~mol} \%)$

$\mathrm{PhSiH}_{3}$ (2 equiv)

${ }^{t} \mathrm{BuO}_{2} \mathrm{H}$ (2 equiv)

PrOH/DCM (1:1 v/v), $24{ }^{\circ} \mathrm{C}$

conversion ${ }^{1}$

cis/trans ratio ${ }^{2}$

$\mathrm{H}$

MOM

OAc

Ns

Piv

$<5$

$>95 \%$

$>95 \%$

$>95 \%$

$<5 \%$<smiles>[R]Oc1ccc2c(c1)OC(c1ccccc1)C[C@H]2c1c(OCc2ccccc2)ccc(C2(C)OCCO2)c1OCc1ccccc1</smiles><smiles>[R]Oc1ccc2c(c1)OC(c1ccccc1)CC2c1c(OCc2ccccc2)ccc(C2(C)OCCO2)c1OCc1ccccc1</smiles>

${ }^{1}$ As judged by crude ${ }^{1} \mathrm{H}$ NMR analysis. ${ }^{2} \mathrm{As}$ judged by ${ }^{1} \mathrm{H}$ NMR analysis of purified material 
Experimental Procedures for the Formal Enantioselective Total Synthesis of (-)-1 and $(-)-3$.

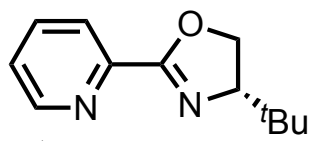<smiles>O=c1ccoc2cc(O)ccc12</smiles>

22
(S) ${ }^{t}$ BuPyOX (6 mol \%)

$\mathrm{Pd}(\mathrm{TFA})_{2}(5 \mathrm{~mol} \%)$ $\mathrm{PhB}(\mathrm{OH})_{2}$ (2 equiv)

$\mathrm{NH}_{4} \mathrm{PF}_{6}(30 \mathrm{~mol} \%)$ $\mathrm{H}_{2} \mathrm{O}$ (5 equiv) DCE, $60{ }^{\circ} \mathrm{C}, 28 \mathrm{~h}$ $(64 \%, 97: 4$ er $)$<smiles>O=C1C[C@H](c2ccccc2)Oc2cc(O)ccc21</smiles>

$(R)-5$

\section{(R)-7-hydroxyflavanone}

A $50 \mathrm{~mL}$ round bottom flask was charged with $\mathrm{Pd}(\mathrm{TFA})_{2}(84.0 \mathrm{mg}, 0.25 \mathrm{mmol})$, (S)-tBuPyOX (62.0 mg, $0.30 \mathrm{mmol})$, ammonium hexafluorophosphate $(250.0 \mathrm{mg}, 1.5$ $\mathrm{mmol})$, and $\mathrm{PhB}(\mathrm{OH})_{2}(1.23 \mathrm{~g}, 10 \mathrm{mmol})$. The flask was sealed and purged with argon and dry dichloroethane $(10 \mathrm{~mL})$ added. The resulting suspension was stirred for 3 minutes at $24{ }^{\circ} \mathrm{C}$ before $22(810 \mathrm{mg}, 5 \mathrm{mmol})$ and water $(0.5 \mathrm{~mL}, 25 \mathrm{mmol})$ were added. An additional portion of dichloroethane $(10 \mathrm{~mL}$ ) was added and the suspension heated to 60 ${ }^{\circ} \mathrm{C}$ and stirred vigorously. After $22 \mathrm{~h}$, the reaction cooled to $24^{\circ} \mathrm{C}$ and contents filtered over a pad of silica gel, which was eluted with EtOAc $(3 \times 50 \mathrm{~mL})$. Silica gel (ca $10 \mathrm{~g})$ was added to the solution and the solvents were removed in vacuo. The free flowing solid was then chromatographed directly (EtOAc-hexanes $1: 10 \rightarrow 2: 5)$ to afford $(R)-5(1.83 \mathrm{~g}, 64 \%$, 96.5:3.5 er) yellow microcrystalline solid. The spectral data for $(R)-5$ matched those previously reported. $\mathrm{S}^{\mathrm{S}}[\mathrm{a}]_{\mathrm{D}}{ }^{24}:(\mathrm{c}=0.98),+73.2^{\circ}$ 

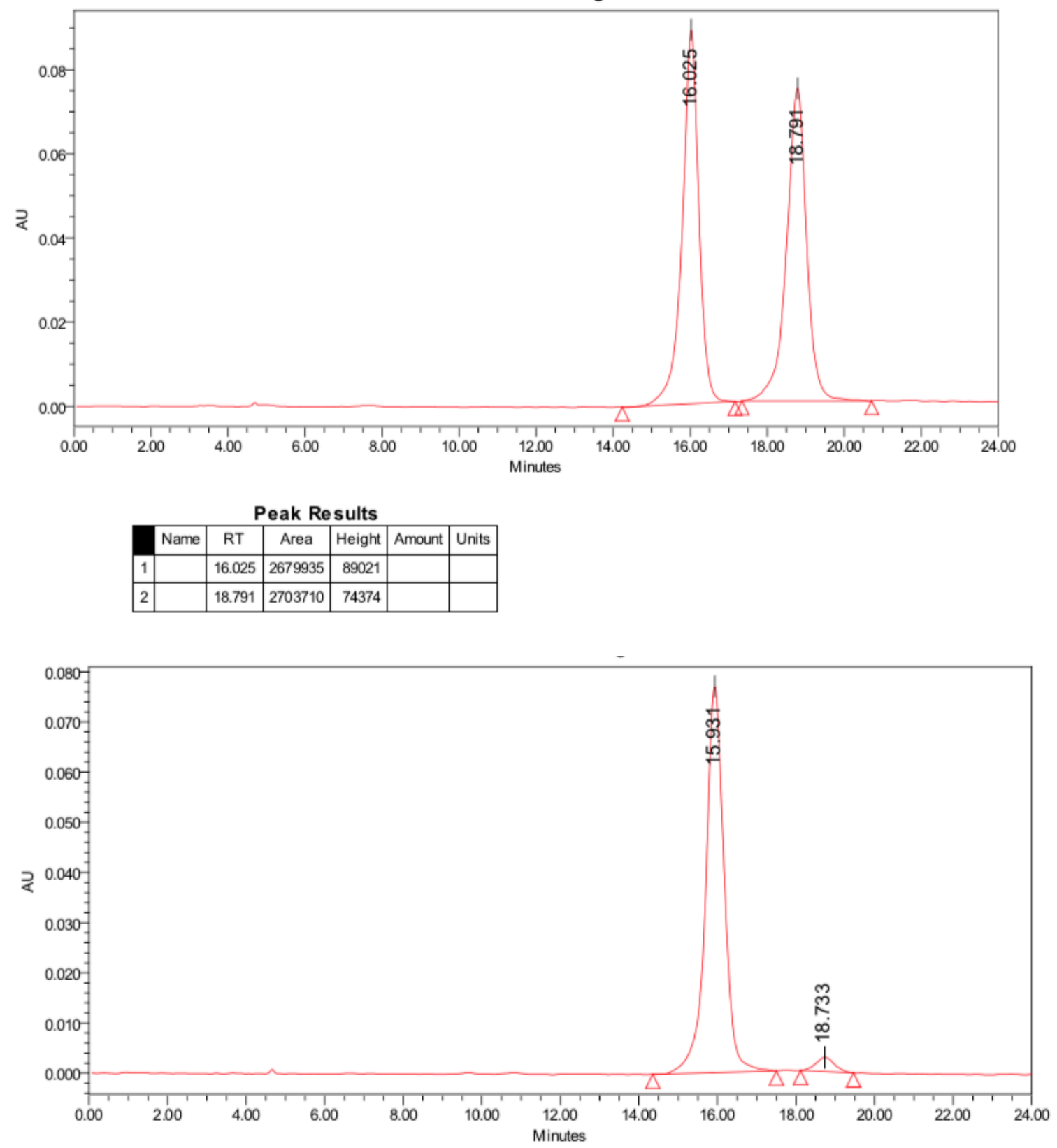

Peak Results
\begin{tabular}{|r|c|c|r|r|r|r|}
\hline & Name & RT & \multicolumn{1}{c|}{ Area } & Height & Amount & Units \\
\hline 1 & & 15.931 & 2528870 & 76969 & & \\
\hline 2 & & 18.733 & 96925 & 2885 & & \\
\hline
\end{tabular}

Figure S5. Chiral HPLC traces of racemic 5 (top trace) and enantioenriched $(R)-\mathbf{5}$ (bottom trace). Phenomenex i-Amylose 1 (4.6 mm x $250 \mathrm{~mm}, 5 \mu \mathrm{M})$, 93:7 hexanesiPrOH@ $1 \mathrm{~mL} / \mathrm{min}$ (96.5:3.5 er) 
<smiles>O=C1C[C@H](c2ccccc2)Oc2cc(O)ccc21</smiles>

$(R)-5$

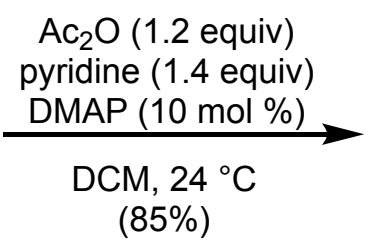

$(85 \%)$<smiles>CC(=O)Oc1ccc2c(c1)O[C@@H](c1ccccc1)CC2=O</smiles>

$(R)-\mathrm{SI}-1$

\section{$(\boldsymbol{R})$-SI-I}

To a solution of $(R)-5(731.5 \mathrm{mg}, 3.0 \mathrm{mmol}, 1$ equiv) in DCM $(32 \mathrm{~mL})$ was added successively acetic anhydride $(0.323 \mathrm{~mL}, 3.6 \mathrm{mmol}, 1.2$ equiv), pyridine $(0.310 \mathrm{~mL}, 4.2$ mmol, 1.5 equiv), and DMAP ( $20 \mathrm{mg}, 0.16 \mathrm{mmol})$. The reaction was stirred for 30 minutes before silica gel (ca. $2 \mathrm{~g}$ ) was added and the volatiles removed in vacuo before being directly loaded in to an empty MPLC cartridge $(25 \mathrm{~g})$ and chromatographed $(40 \mathrm{~g}$ column, $60 \mathrm{~mL} / \mathrm{min}, 10 \rightarrow 40 \%$ ethyl acetate-hexanes) to give acetate $(R)-5(758 \mathrm{mg}$, $85 \%, 96.5: 3.5$ er) as a colorless microcrystalline solid. The spectral data of $(R)$-SI-I matched those previously reported. ${ }^{\mathrm{S} 2}[\mathrm{a}]_{\mathrm{D}}{ }^{24}:(\mathrm{c}=0.66),+43.0^{\circ}$ 


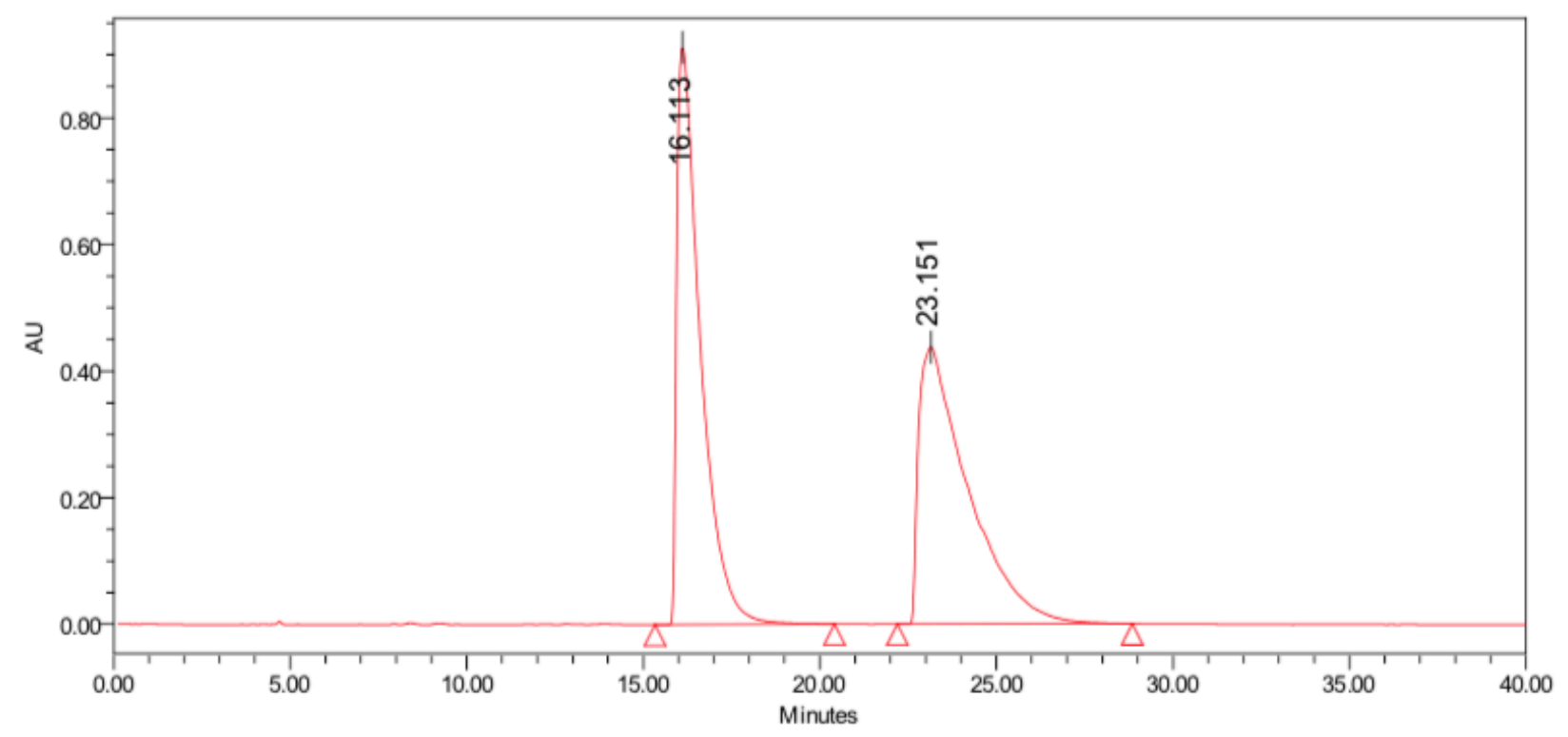

Peak Results

\begin{tabular}{|c|c|c|c|c|c|c|}
\hline & Name & RT & Area & Height & Amount & Units \\
\hline 1 & & 16.113 & 42503597 & 912017 & & \\
\hline 2 & & 23.151 & 42468004 & 437764 & & \\
\hline
\end{tabular}

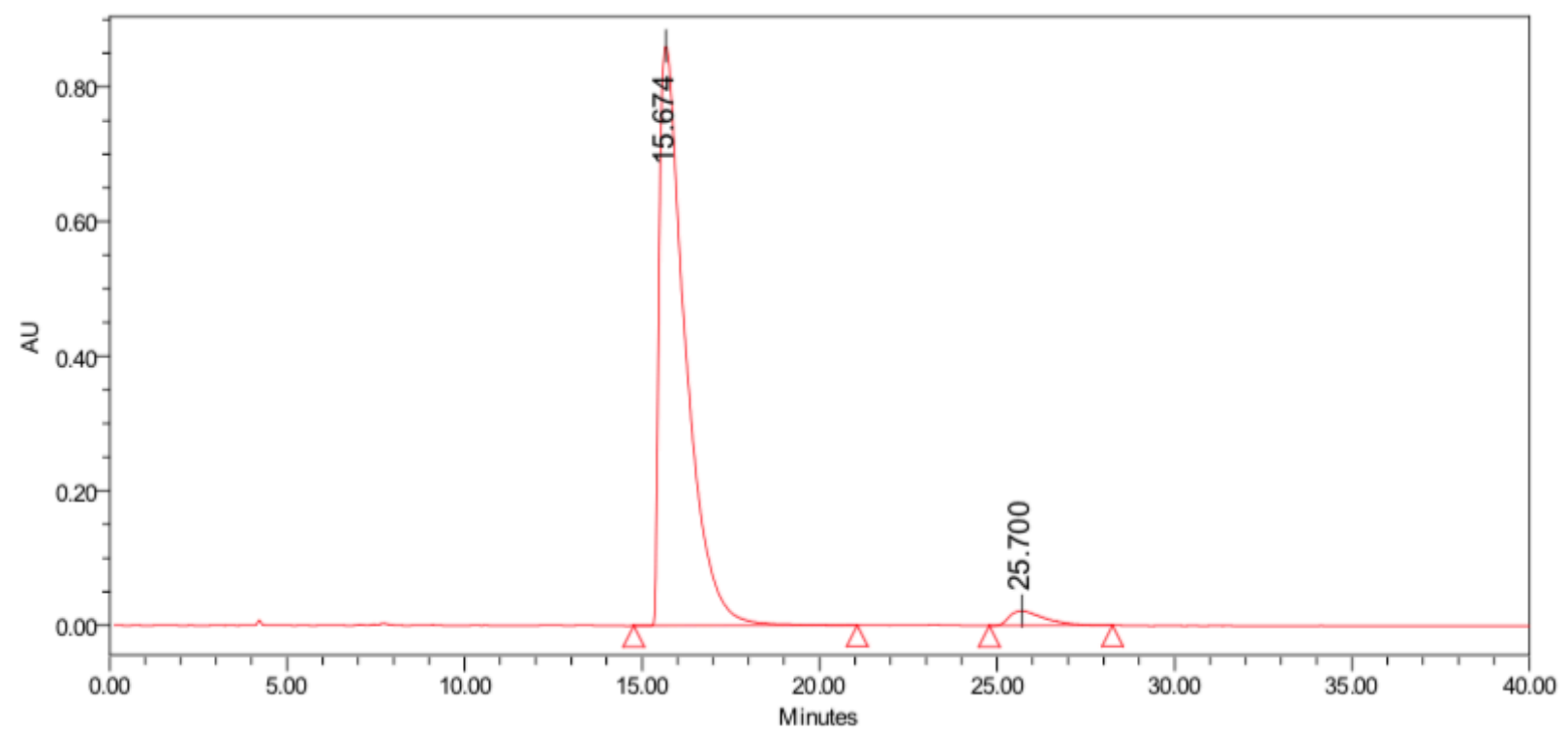

Peak Results

\begin{tabular}{|r|c|c|r|r|r|r|}
\hline & Name & RT & \multicolumn{1}{|c|}{ Area } & Height & Amount & Units \\
\hline 1 & & 15.674 & 43952330 & 861202 & & \\
\hline 2 & & 25.700 & 1491630 & 21718 & & \\
\hline
\end{tabular}

Figure S6: Chiral HPLC traces of racemic SI-I (top trace) and enantioenriched SI-I (bottom trace). Phenomenex i-amylose 1 (4.6 mm x $250 \mathrm{~mm}, 5 \mu \mathrm{M})$, 85:15 hexanesPrOH @ 1 mL/min. (96.5:3.5 er) 
<smiles>CC(=O)Oc1ccc2c(c1)O[C@@H](c1ccccc1)CC2=O</smiles>

(R)-SI-1

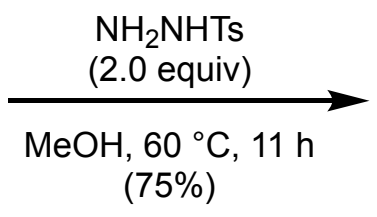

$(75 \%)$<smiles>CC(=O)Oc1ccc2c(c1)O[C@@H](c1ccccc1)CC2=N[AsH2]</smiles>

\section{$N$-Tosylhydrazone $(R)-8$ :}

To a $100 \mathrm{~mL}$ round-bottom flask equipped with a magnetic stir-bar was added $(R)$ SI-1 (645 mg, $2.28 \mathrm{mmol}, 1$ equiv) and $\mathrm{MeOH}$ (28 mL). To the stirred solution was added p-tosylhydrazide (797 mg, $4.35 \mathrm{mmol}, 1.9$ equiv) and the flask then heated to $60{ }^{\circ} \mathrm{C}$ under a static atmosphere of nitrogen. After $11 \mathrm{~h}$, the solution slowly cooled to $4^{\circ} \mathrm{C}$ over the course of 1 hour. The precipitate was collected in a large Kiriyama filter to yield $(R)-8(767$ $\mathrm{mg}, 75 \%, 95: 5 \mathrm{er}$ ) as a colorless microcrystalline solid. The material was azeotropicallydried from benzene $(3 \times 10 \mathrm{~mL})$ before being used in the next step. The spectral data for $(R)-8$ matched those for racemic $8 .[a]_{D}^{24}:(c=0.46),-84.4^{\circ}$ 

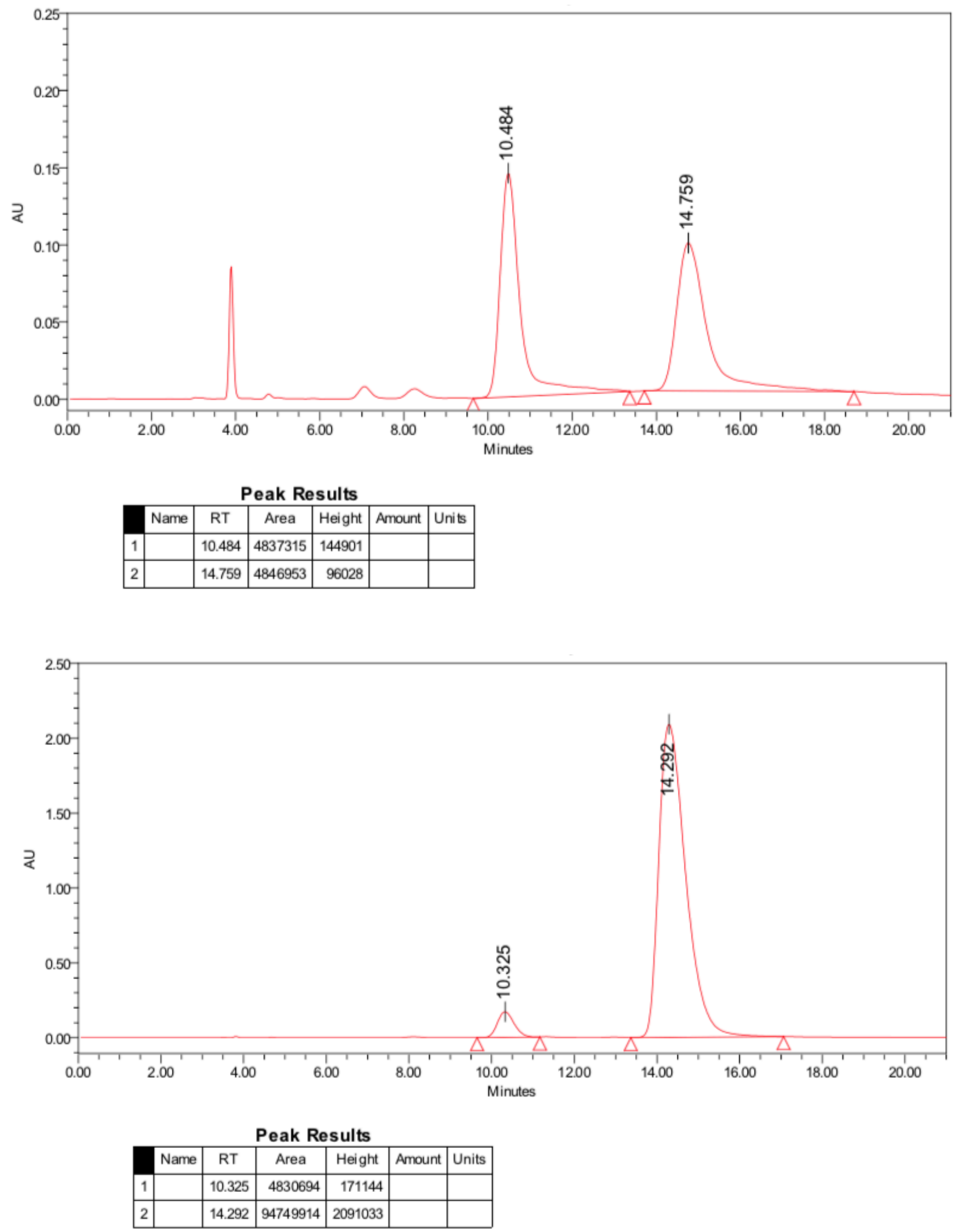

Figure S7: Chiral HPLC traces of racemic 8 (top trace) and enantioenriched $(R)$-8 (bottom trace). Phenomenex i-Amylose 1 (4.6 mm x 250 mm, $5 \mu \mathrm{M}), 70: 30$ hexanes-iPrOH @ 1 $\mathrm{mL} / \mathrm{min}$. (95:5 er) 
<smiles>CC(=O)Oc1ccc2c(c1)O[C@H](c1ccccc1)CC2=N[AsH3-]</smiles>

(R)-8 (95:5 er)<smiles>CC1(c2ccc(OCc3ccccc3)c(I)c2OCc2ccccc2)OCCO1</smiles>

XPhos Pd G3 (20 mol \%) XPhos $(20 \mathrm{~mol} \%)$

$\mathrm{K}_{2} \mathrm{CO}_{3}$ (5.0 equiv)

$\mathrm{PhF}, 85^{\circ} \mathrm{C}, 90 \mathrm{~min}$

$(59 \%, 1: 1.8 \mathrm{dr})$<smiles>CC(=O)Oc1ccc2c(c1)O[C@H](c1ccccc1)C=C2c1c(OCc2ccccc2)ccc(C2(C)OCCO2)c1OCc1ccccc1</smiles>

\section{enantioenriched benzopyran 9}

A 1.5 dram vial equipped with stir-bar was added $8(80.0 \mathrm{mg}, 0.176 \mathrm{mmol}, 1.0$ equiv) XPhos Pd G3 (27.2 mg, $0.035 \mathrm{mmol}, 20 \mathrm{~mol} \%$ ), XPhos (17.2 mg, $0.036 \mathrm{mmol}, 20 \mathrm{~mol}$ $\%)$, followed by dry $\mathrm{PhF}(1.6 \mathrm{~mL})$. The suspension was stirred under argon for 5 minutes before $\mathrm{K}_{2} \mathrm{CO}_{3}(145.6 \mathrm{mg}, 1.05 \mathrm{mmol}, 6$ equiv) and 7 (355.6 g, $0.70 \mathrm{mmol}, 4$ equiv). The vial was sealed before being left under a static atmosphere of argon and heated to $85^{\circ} \mathrm{C}$ for 60 minutes with vigorous stirring. After the reaction was complete, it was cooled to 24 ${ }^{\circ} \mathrm{C}$ and filtered over a pad of silica gel. The silica gel pad was washed with EtOAc (5 $\mathrm{x}$ $150 \mathrm{~mL}$ ) and the washes combined before being concentrated in vacuo to give an orange oil. The oil was chromatographed (MPLC, $12 \mathrm{~g}$ column, $30 \mathrm{~mL} / \mathrm{min}$ hexanes $\rightarrow 40 \%$ EtOAc/hexanes to give 9 as a light-yellow foam $(63.6 \mathrm{mg}, 56 \%$, (Major atropisomer: $93: 7$ er. Minor atropisomer: $91.5: 8.5$ er). The spectral data for enantioenriched 9 were identical to that of racemic 9

Note 1: Complete baseline separation couldn't be achieved for the major atropisomer in our hands. However, both atropisomers seem to have virtually the same enantioenrichment.

Note 2: Optical rotation data were not taken for 9 as it was comprised of inseparable atropisomers. 


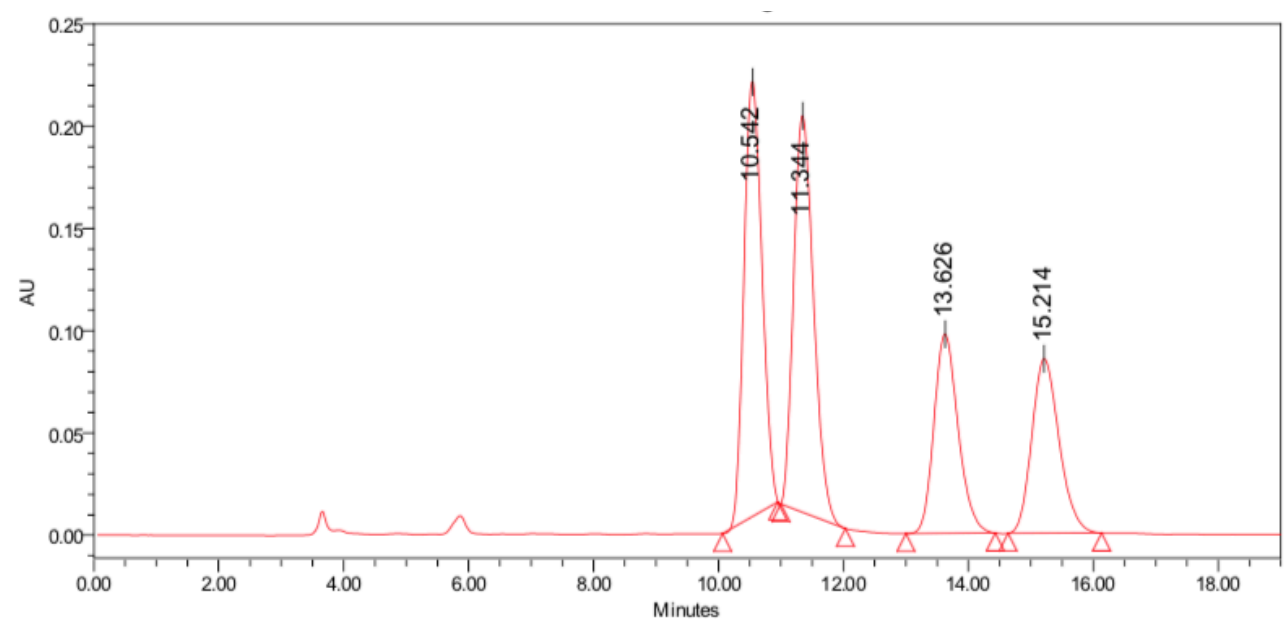

\begin{tabular}{|c|c|c|c|c|c|c|}
\multicolumn{7}{|c|}{ Peak Results } \\
\hline 1 & Name & RT & Area & Height & Amount & Units \\
\hline 2 & & 10.542 & 4251672 & 212945 & & \\
\hline 3 & & 11.344 & 4308237 & 194020 & & \\
\hline 4 & & 13.626 & 2622137 & 97534 & & \\
\hline
\end{tabular}

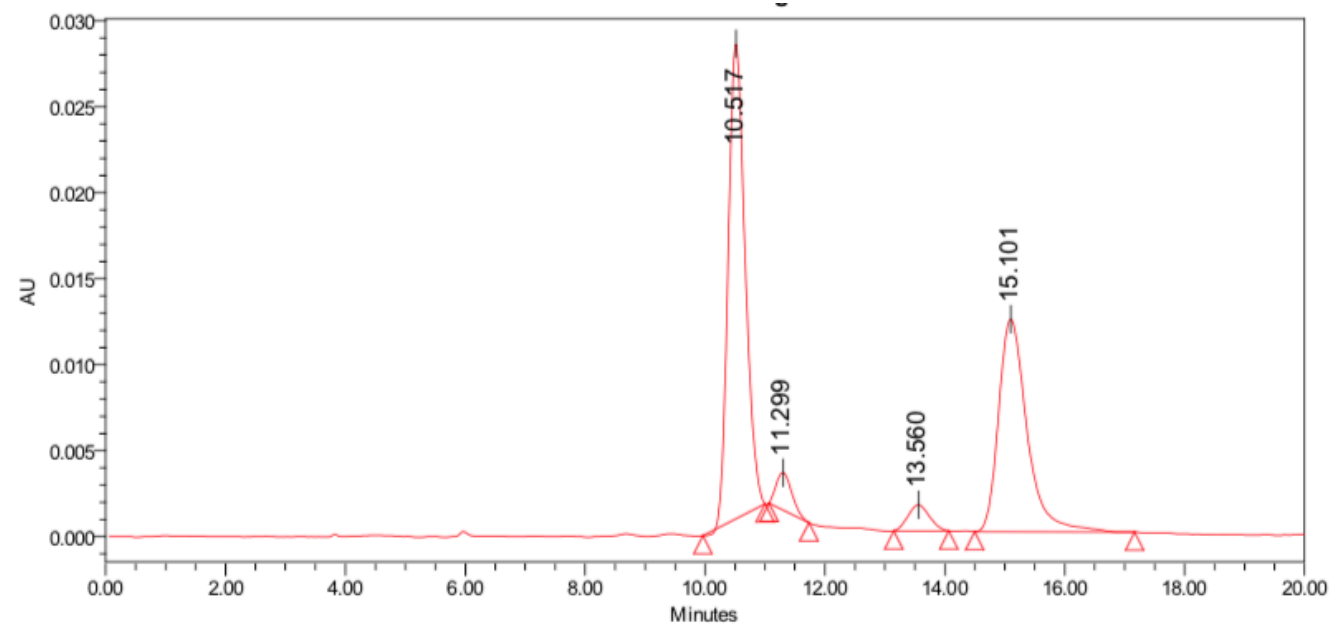

Peak Results

\begin{tabular}{|r|r|c|r|r|r|r|}
\hline & Name & RT & Area & Height & Amount & Units \\
\hline 1 & & 10.517 & 542093 & 27627 & & \\
\hline 2 & & 11.299 & 39760 & 2170 & & \\
\hline 3 & & 13.560 & 37501 & 1516 & & \\
\hline 4 & & 15.101 & 408421 & 12353 & & \\
\hline
\end{tabular}

Figure S8: Chiral HPLC traces of racemic 9 (top trace) and enantioenriched 9 (bottom trace). Phenomenex i-Amylose 1 (4.6 mm x $250 \mathrm{~mm}, 5 \mu \mathrm{M}), 80: 3$ hexanes-iPrOH @ 0.83 $\mathrm{mL} / \mathrm{min}$. (Major atropisomer: 93:7 er. Minor atropisomer: 91.5:8.5 er) 
<smiles>CC(=O)Oc1ccc2c(c1)O[C@H](c1ccccc1)C=C2c1c(OCc2ccccc2)ccc(C2(C)OCCO2)c1OCc1ccccc1</smiles>

(R)-9

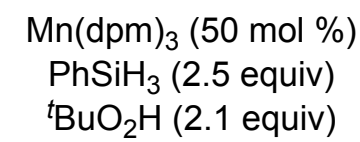

$(58 \%, 1.5: 110 a: 10 b)$<smiles>CC(=O)Oc1ccc2c(c1)O[C@@H](c1ccccc1)CC2c1c(OCc2ccccc2)ccc(C2(C)OCCO2)c1OCc1ccccc1</smiles>

$10 \mathrm{a}$<smiles>CC(=O)Oc1ccc2c(c1)O[C@@H](c1ccccc1)C[C@H]2c1c(OCc2ccccc2)ccc(C2(C)OCCO2)c1OCc1ccccc1</smiles>

$10 \mathrm{~b}$

89:11 er

(99:1 er after recrystallization)

\section{Dihydropyrans 10a and 10b:}

To a stirred solution of $(R)-9$ (17 mg, $0.026 \mathrm{mmol}, 1$ equiv) in dry $\mathrm{PhCF}_{3} / \mathrm{PrOH}(1.0 \mathrm{~mL}$, $3: 1 \mathrm{v} / \mathrm{v})$ was added $\mathrm{PhSiH}_{3}(7.0 \mu \mathrm{L}, 0.065 \mathrm{mmol}, 2.5$ equiv) and tbutyl hydroperoxide (10 $\mu \mathrm{L}, 5.5 \mathrm{M}$ in decane, $0.055 \mathrm{mmol}, 2.1$ equiv). The solution was sparged for 5 minutes with dry argon before $\mathrm{Mn}(\mathrm{dpm})_{3}(8.0 \mathrm{mg}, 0.013 \mathrm{mmol}, 0.5$ equiv) was added in one portion. The solution was then sparged for 10 seconds with dry argon before being left under a static atmosphere of argon and stirred at $24{ }^{\circ} \mathrm{C}$ for $40 \mathrm{~min}$. After completion (as judged by ESI-MS), silica gel (ca. $0.5 \mathrm{~g}$ ) was added and the suspension concentrated to a freeflowing powder, which was chromatographed directly by MPLC (4 g column, $13 \mathrm{~mL} / \mathrm{min}$, $10 \% \rightarrow 40 \%$ ethyl acetate/hexane) to give $10 \mathrm{a}$ and $10 \mathrm{~b}(9.9 \mathrm{mg}, 58 \%)$ as a $1.5: 1$ mixture of trans/cis isomers and as a colorless oil. The mixture was separated by, preparative reverse-phase HPLC (21.2 x $100 \mathrm{~mm}$ Phenomenex Gemini column (5 $\mu \mathrm{m} \mathrm{C}_{18} 110 \AA, 21.2$ $x 100 \mathrm{~mm}$ ), $65 \% \mathrm{MeCN}$ in $\mathrm{H}_{2} \mathrm{O} \rightarrow 100 \% \mathrm{MeCN}$ over 15 minutes, $20 \mathrm{~mL} / \mathrm{min}$ ) to give10a (5.8 mg, 89:11 er, colorless microcrystalline solid) and $10 \mathrm{~b}(2.1 \mathrm{mg}$, ca. $92.5: 7.5 \mathrm{er}$, colorless solid). The spectral data for enantioenriched 10a and 10b were identical to racemic 10a and 10b. Optical Rotation: $[\alpha]_{D^{24}}$ 10a $\left(89: 11\right.$ er material: $[a]^{24}:(c=0.29)$, $\left.-5.5^{\circ}\right)[\alpha]_{D^{24}} 10 \mathrm{~b}$ (ca. 92.5:7.5 er material: $\left.[\mathrm{a}]_{\mathrm{D}^{24}}:(\mathrm{c}=0.12),+23.3^{\circ}\right)$

Note 1: The enantiopurity of $10 \mathrm{~b}$ could not be definitively assigned due to a closely eluting impurity in the chiral HPLC spectrum, even after both normal-phase and reverse-phase purification.

Note 2: For recrystallization: 10a was dissolved in hot hexanes/iPrOH (ca 10:1 v/v) and allowed to cool slowly to room temperature. After 6 hours, crystals formed and the was mother liquor decanted. Chiral HPLC analysis of the recrystallized material showed a significant upgrade of enantiopurity (99:1 er)

Note 3: Attempts at recrystallization of $\mathbf{1 0 b}$ were unsuccessful. 


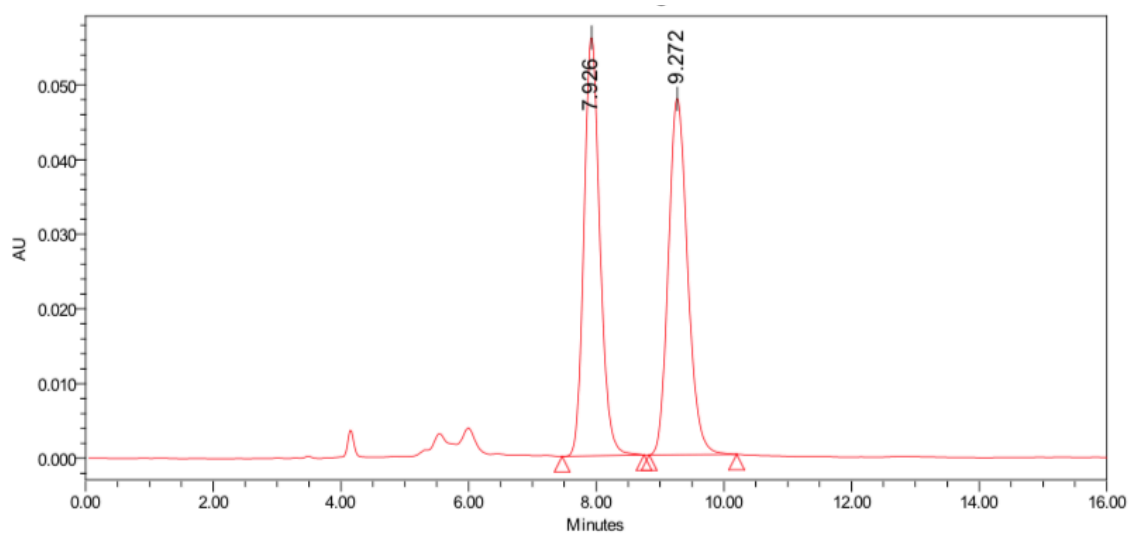

Peak Results

\begin{tabular}{|l|l|l|l|l|l|}
\hline Name & RT & Area & Height & Amount & Units \\
\hline
\end{tabular}

\begin{tabular}{l|l|l|l|l|l|}
\hline 1 & & 7.926 & 970553 & 56129 \\
\hline 2 & & 9272 & 995344 & 47702 \\
\hline
\end{tabular}

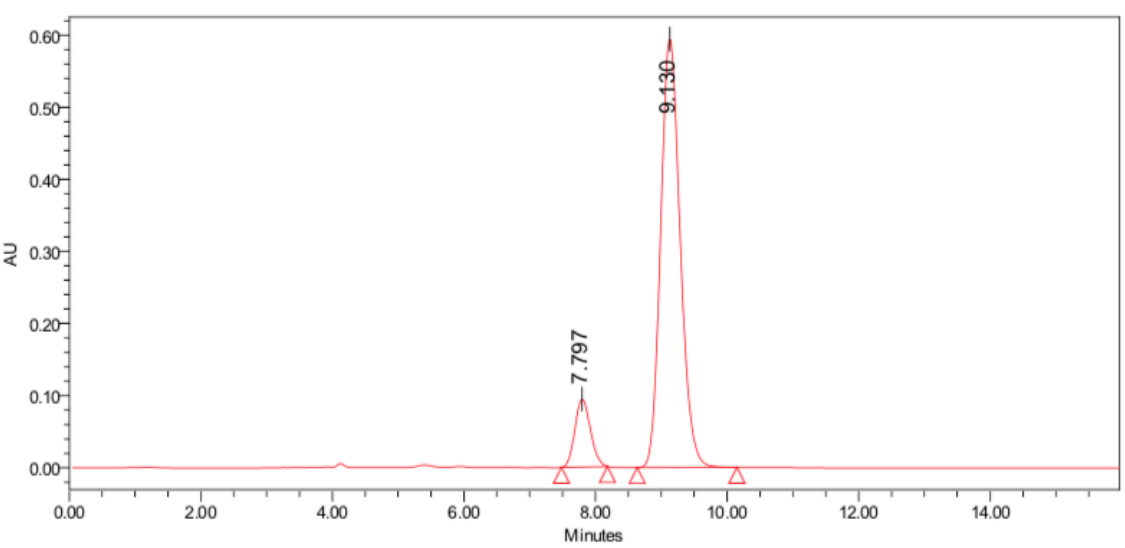

Peak Results

\begin{tabular}{|l|l|l|l|l|l|}
\hline Name & RT & Area & Height & Amount & Units \\
\hline
\end{tabular}

\begin{tabular}{|r|r|r|r|r|}
\hline 1 & & 7.797 & 1545578 & 94218 \\
\hline 2 & & 9.130 & 12239355 & 594994 \\
\hline
\end{tabular}

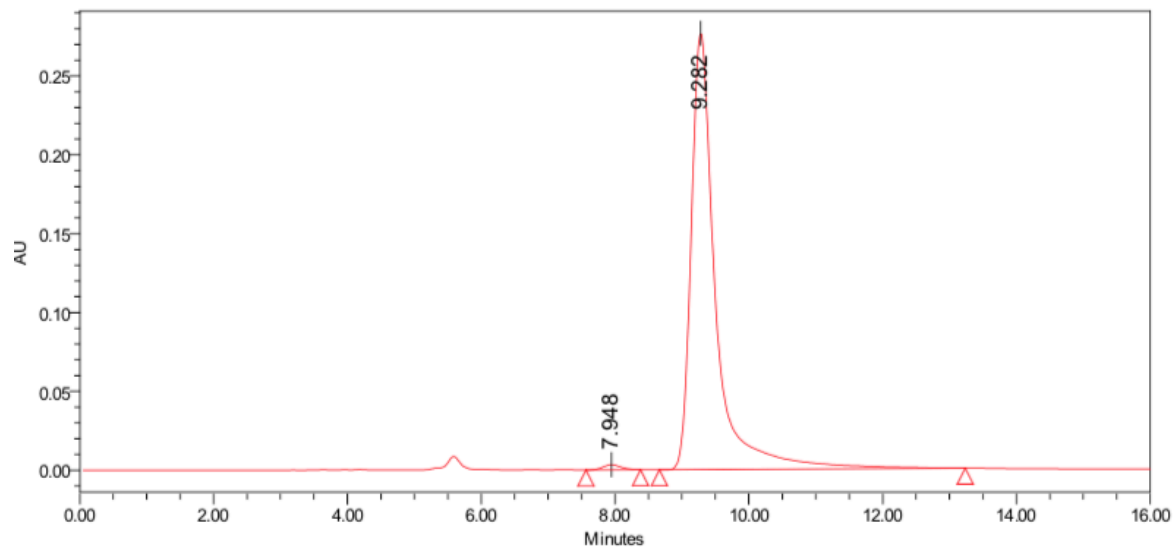

Peak Results

\begin{tabular}{l|l|l|l|l|l|} 
Name & RT & Area & Height & Amount & Units \\
\hline
\end{tabular}

\begin{tabular}{|r|r|r|r|r|}
\hline 1 & & 7.948 & 61910 & 3334 \\
\hline
\end{tabular}

\begin{tabular}{|l|l|l|l|l|}
\hline 2 & & 9.282 & 7045968 & 276766 \\
\hline
\end{tabular} 
Figure S9: Chiral HPLC traces of racemic 10a (top trace) and enantioenriched 10a (middle trace) and recrystallized 10a (bottom trace) Phenomenex i-Amylose 1 (4.6 $\mathrm{mm} \mathrm{x}$ $250 \mathrm{~mm}, 5 \mu \mathrm{M}), 85: 15$ hexanes-iPrOH @ $1.0 \mathrm{~mL} / \mathrm{min}$. (89:11 er) 


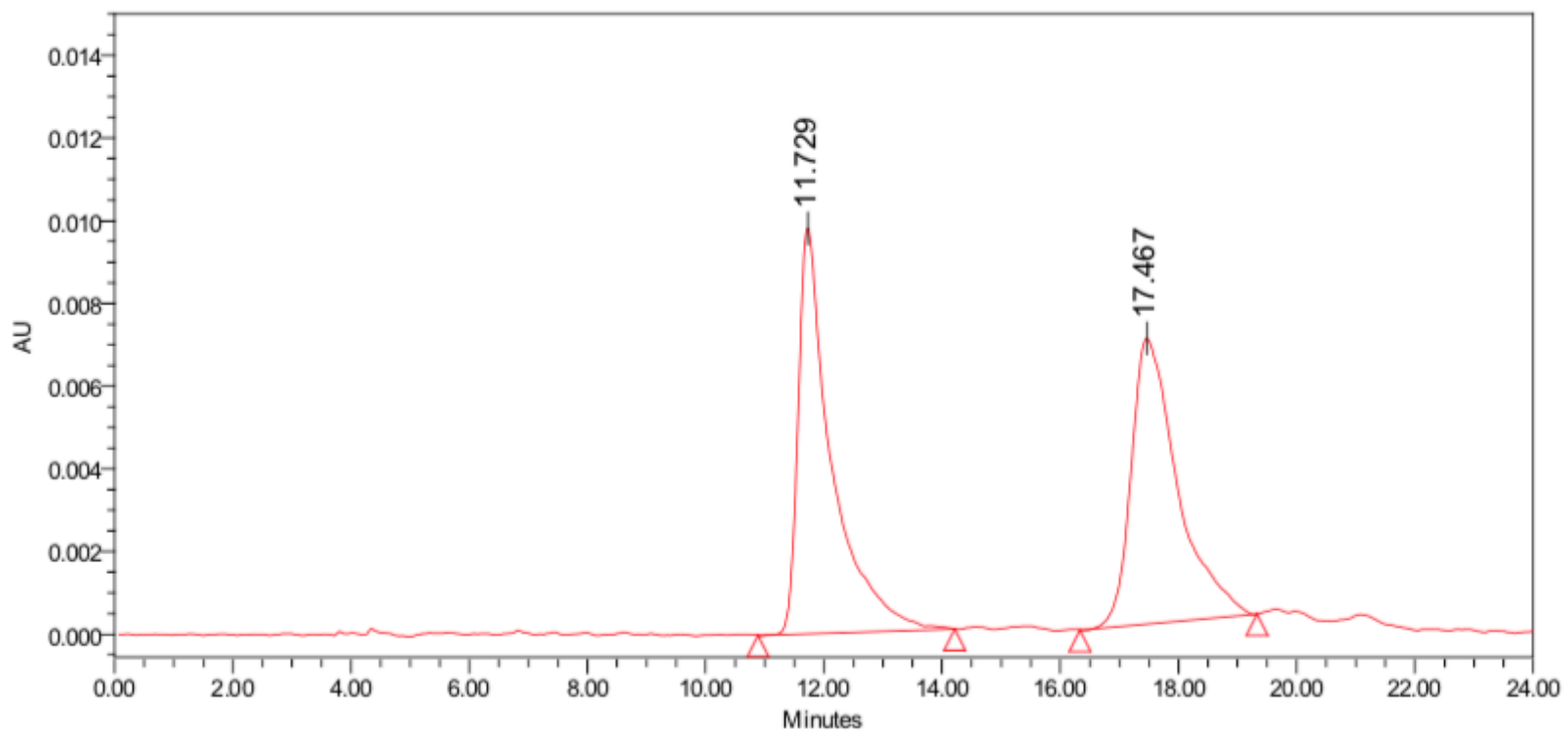

Peak Results

\begin{tabular}{|r|c|c|c|r|r|r|}
\hline & Name & RT & Area & Height & Amount & Units \\
\hline 1 & & 11.729 & 385365 & 9812 & & \\
\hline 2 & & 17.467 & 376124 & 6907 & & \\
\hline
\end{tabular}

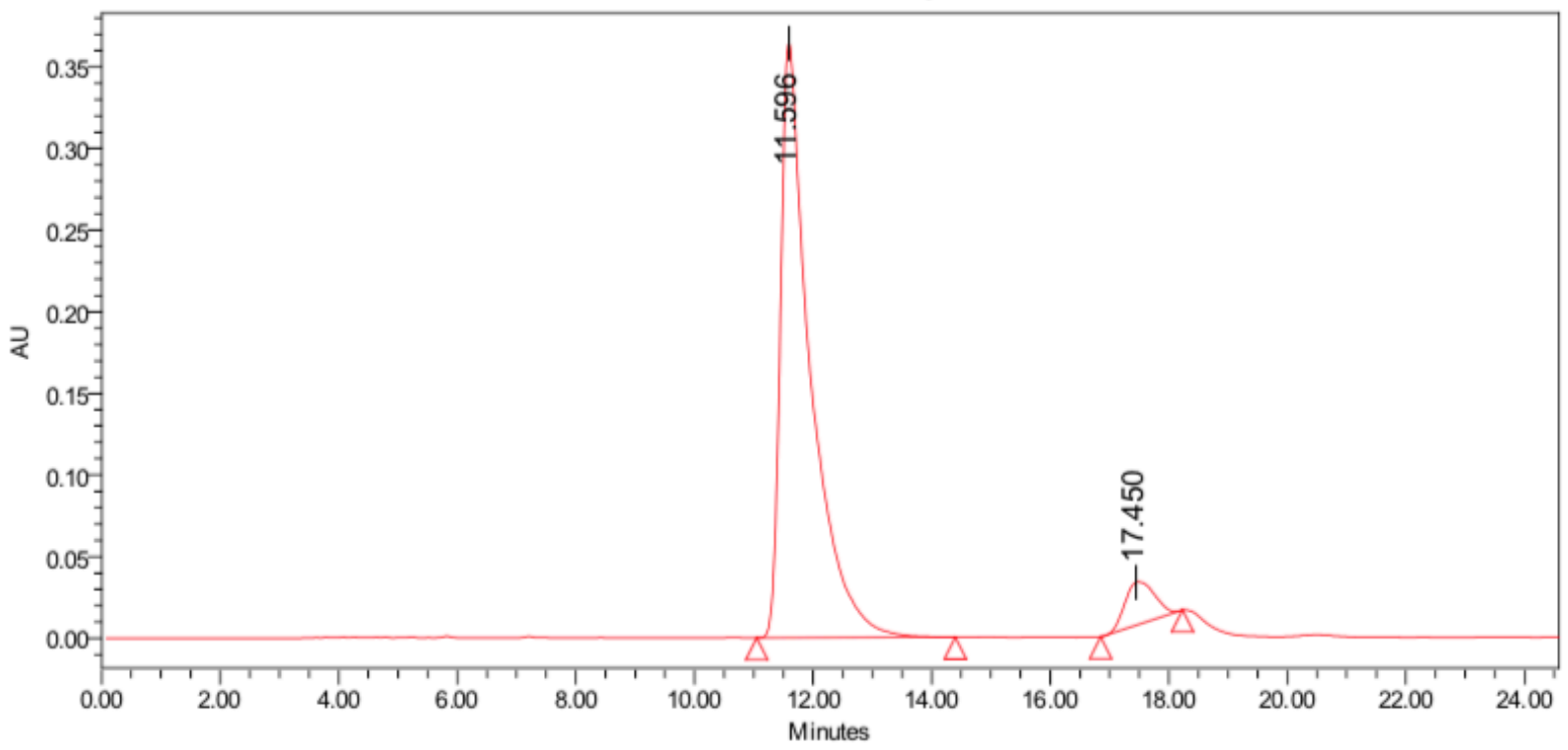

\begin{tabular}{|r|c|c|r|r|r|r|}
\multicolumn{7}{|c|}{ Peak Results } \\
\hline 1 & Name & RT & Area & Height & Amount & Units \\
\hline 2 & & 11.596 & 12735681 & 364422 & & \\
\hline
\end{tabular}

Figure S10: Chiral HPLC traces of racemic 10b (top trace) and enantioenriched 10a (bottom trace). Phenomenex i-Amylose 1 (4.6 mm x $250 \mathrm{~mm}, 5 \mu \mathrm{M})$, 80:3 hexanesiPrOH@ 0.83 mL/min. 


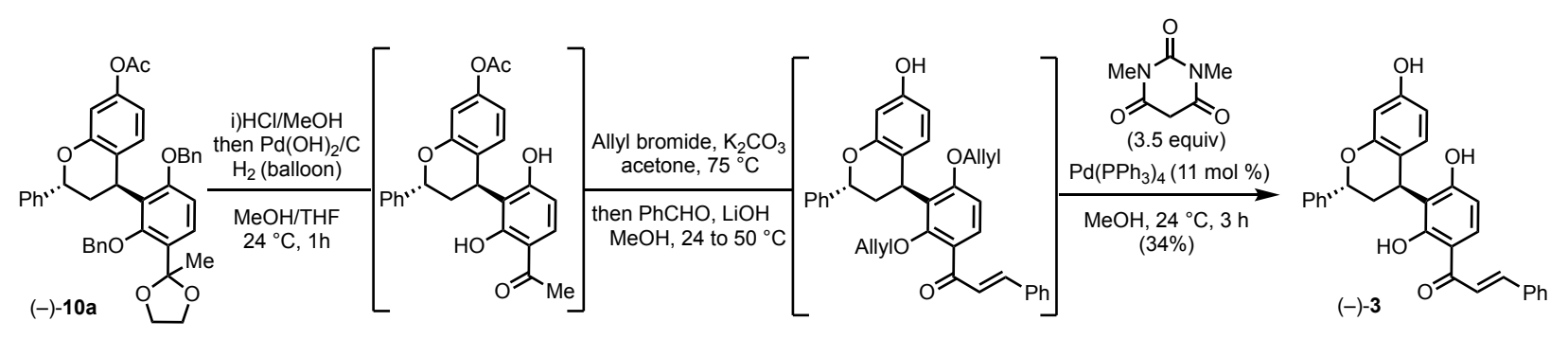

$(-)$-Caesalpinflavan B ((-)-3):

Step 1. To a solution of enantioenriched dihydropyran (-)-10a $(30.0 \mathrm{mg}, 0.046$ $\mathrm{mmol})$ in $\mathrm{MeOH}(3.0 \mathrm{~mL})$ was added $10 \%$ aq. $\mathrm{HCl}(10.0 \mu \mathrm{L})$. The solution stirred at $24^{\circ} \mathrm{C}$ for 20 minutes before being sparged with dry argon for 15 minutes, followed by evacuation under vacuum (ca. 15 torr) for 5 minutes. THF ( $3 \mathrm{~mL}$ ) was then added and the solution then sparged with hydrogen gas for 5 minutes before being placed under a static atmosphere of hydrogen gas (Note 1). To the resulting solution was added Pearlman's catalyst (20.0 mg, Note 2). The resulting suspension was sparged with hydrogen gas for 5 minutes before being placed under an atmosphere of static hydrogen and stirred vigorously for 50 minutes. After the reaction was complete, it was rapidly filtered through a plug of Celite, eluting with ethyl acetate $(5 \mathrm{~mL})$. The resulting solution was concentrated under vacuum to give enantioenriched bisphenol 18a $(50.7 \mathrm{mg})$, which was used crude in the next step.

Step 2.To a solution of crude enantioenriched bisphenol $18 \mathrm{a}(50.7 \mathrm{mg})$ in acetone $(1.5 \mathrm{~mL})$ in a $10 \mathrm{~mL}$ microwave tube was added allyl bromide $(20.0 \mu \mathrm{L}, 0.23 \mathrm{mmol})$ and potassium carbonate $(20.0 \mathrm{mg}, 0.144 \mathrm{mmol})$. The tube was then sealed and placed under an atmosphere of dry nitrogen before being heated in a microwave at $85^{\circ} \mathrm{C}$ for 6 hours. The suspension was then cooled to $24^{\circ} \mathrm{C}$ and volatiles removed under vacuum. $\mathrm{MeOH}$ $(1 \mathrm{~mL})$ was then added to the tube and the suspension stirred for 10 minutes before benzaldehyde $(40.0 \mu \mathrm{L}, 0.38 \mathrm{mmol})$ and lithium hydroxide monohydrate $(40.0 \mathrm{mg}, 0.95$ $\mathrm{mmol}$ ) were added. The tube was then heated in an aluminum heating block at $50^{\circ} \mathrm{C}$ for 2 hours. After being cooled to $24^{\circ} \mathrm{C}$, the suspension was quenched with $5 \mathrm{~mL}$ saturated aqueous ammonium chloride. The mixture was extracted with ethyl acetate $(5 \times 5 \mathrm{~mL})$ and the combined organic phases washed with saturated brine $(5 \mathrm{~mL})$, dried over sodium sulfate, filtered, and concentrated in vacuo (Note 3). After drying under high vacuum 6 hours, the crude enantioenriched phenol $19 \mathrm{a}(25.5 \mathrm{mg})$ was used crude in the next step.

Step 3. To a dry $20 \mathrm{~mL}$ vial containing 1,3-dimethyl barbituric acid (30.0 mg, 0.192 $\mathrm{mmol}),\left[\mathrm{Pd}\left(\mathrm{PPh}_{3}\right)_{4}\right](10.5 \mathrm{mg}, 0.0091 \mathrm{mmol})$, crude phenol 19a $(25.5 \mathrm{mg})$ and a magnetic stir-bar was added dry THF $(5 \mathrm{~mL})$. The bright yellow solution was stirred for 15 mins before being rapidly filtered through a short plug of silica gel, which was eluted with ethyl acetate $(10 \mathrm{~mL})$. The resulting solution was concentrated under vacuum and chromatographed directly by MPLC (4 g column, $13 \mathrm{~mL} / \mathrm{min}, 10 \% \rightarrow 60 \%$ ethyl acetate/hexane) to give caesalpinflavan B $(8.7 \mathrm{mg}, 41 \%$ over 3 steps, $>99: 1 \mathrm{er})$ as a yellow amorphous solid. The analytical data for (-)-3 matched those obtained for racemic 3: Optical Rotation: $[\mathrm{a}]_{\mathrm{D}}^{24}:(\mathrm{c}=0.20, \mathrm{MeOH}),-38.0^{\circ}$. 
Note 1: The sparging/vacuum cycle is to degas $\mathrm{HCl}$ from the reaction in order to avoid over reduction of the flavan $\mathrm{C}$ ring $\mathrm{C}-\mathrm{O}$ bond.

Note 2: We observed a marked effect of the age of Pearlman's catalyst and selectivity for our desired reaction; Fresh bottles of Pearlman's catalyst (from Millipore Sigma) gave mixtures of the desired bisphenol and overreduction, presumably to a trisphenol where the $\mathrm{C}-\mathrm{O}$ bond in the dihydropyran ring had been cleaved. Older bottles (ca. 15-20 years old, Aldrich) were less active and were slower to cleave this more hindered $\mathrm{C}-\mathrm{O}$ bond. However, if the reaction is not filtered through Celite quickly after the removal of both benzyl groups, $\mathrm{C}-\mathrm{O}$ bond cleavage of the flavanoid C-ring begins to occur (as judged by MS analysis of aliquots).

Note 3: High vacuum was ca. 200 mTorr for 12 hours. This removed the majority of remaining benzaldehyde from the Claisen-Schmidt reaction (as judged by ${ }^{1} \mathrm{H}$ NMR)

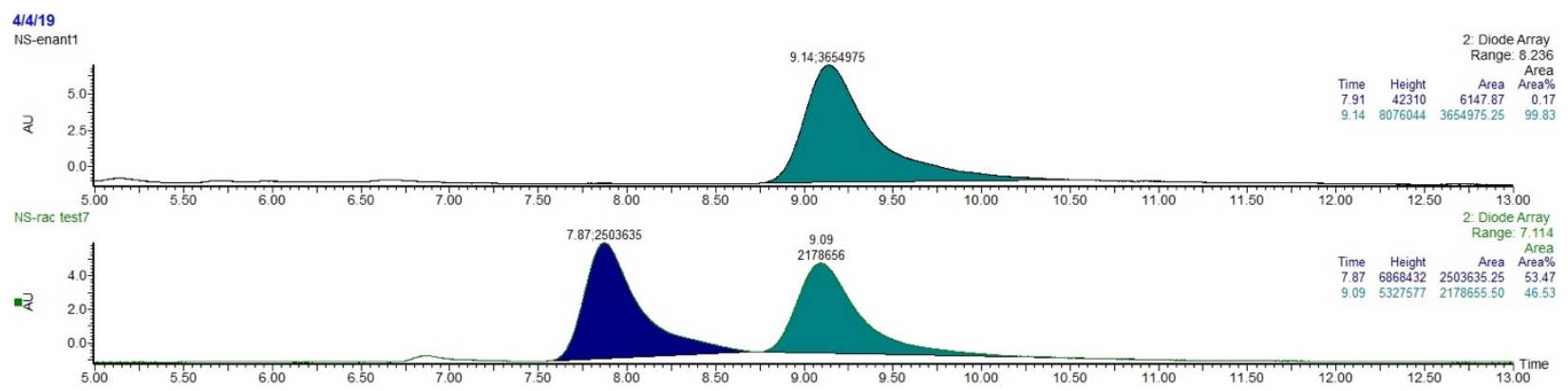

Figure S11: Chiral UPLC traces of racemic (-)-3 (top trace) and racemic 3 (bottom trace). Phenomenex Lux Cellulose-2 (4.6 mm x $150 \mathrm{~mm}, 3 \mu \mathrm{M})$, 40:60(0.2\% formic acid) $\mathrm{MeCN}-$ Water @ $0.7 \mathrm{~mL} / \mathrm{min}$. 
NMR Spectra of New Compounds 


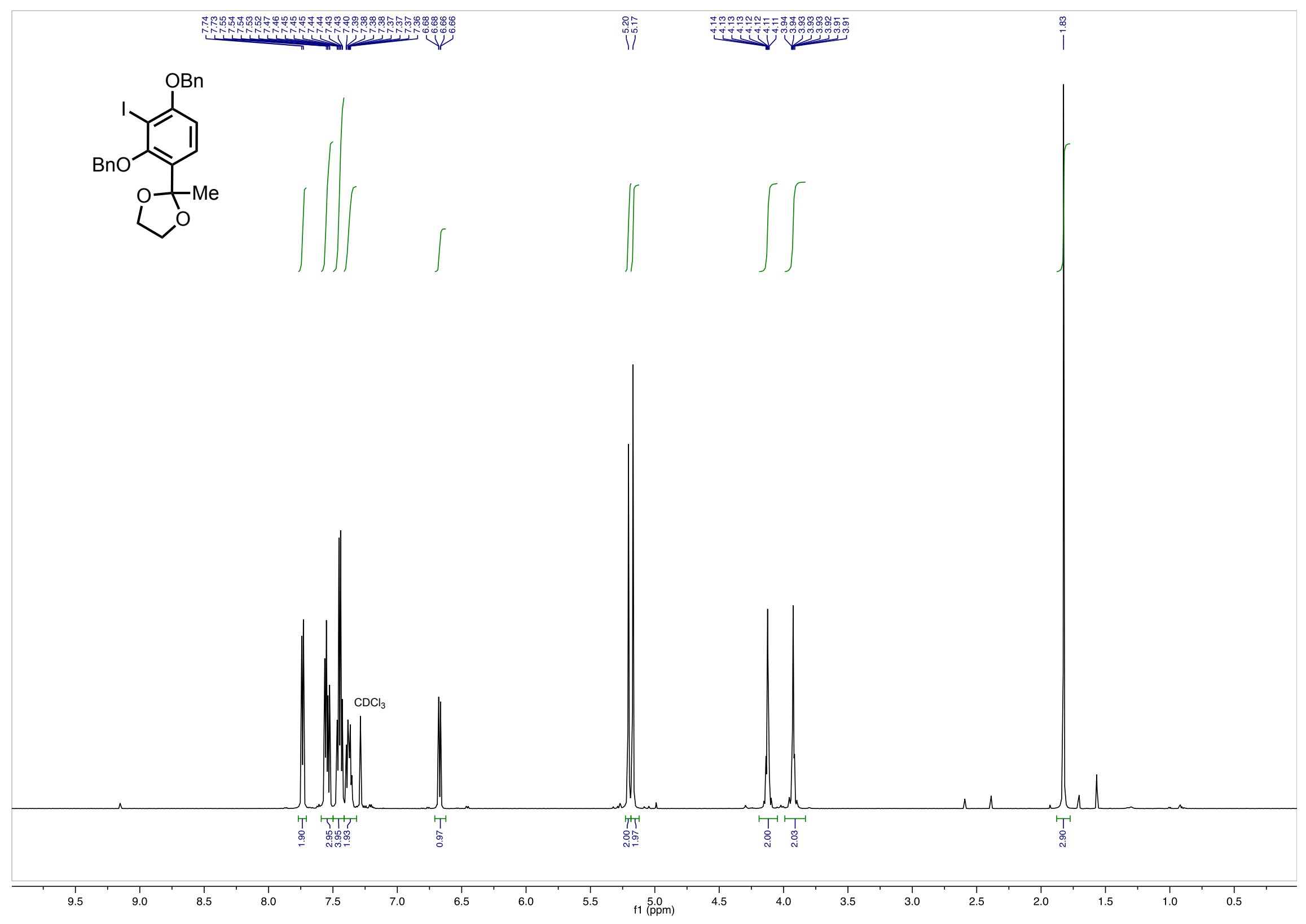

Figure S12. ${ }^{1} \mathrm{H}$ NMR $(600 \mathrm{MHz}$, chloroform-d), 7. 


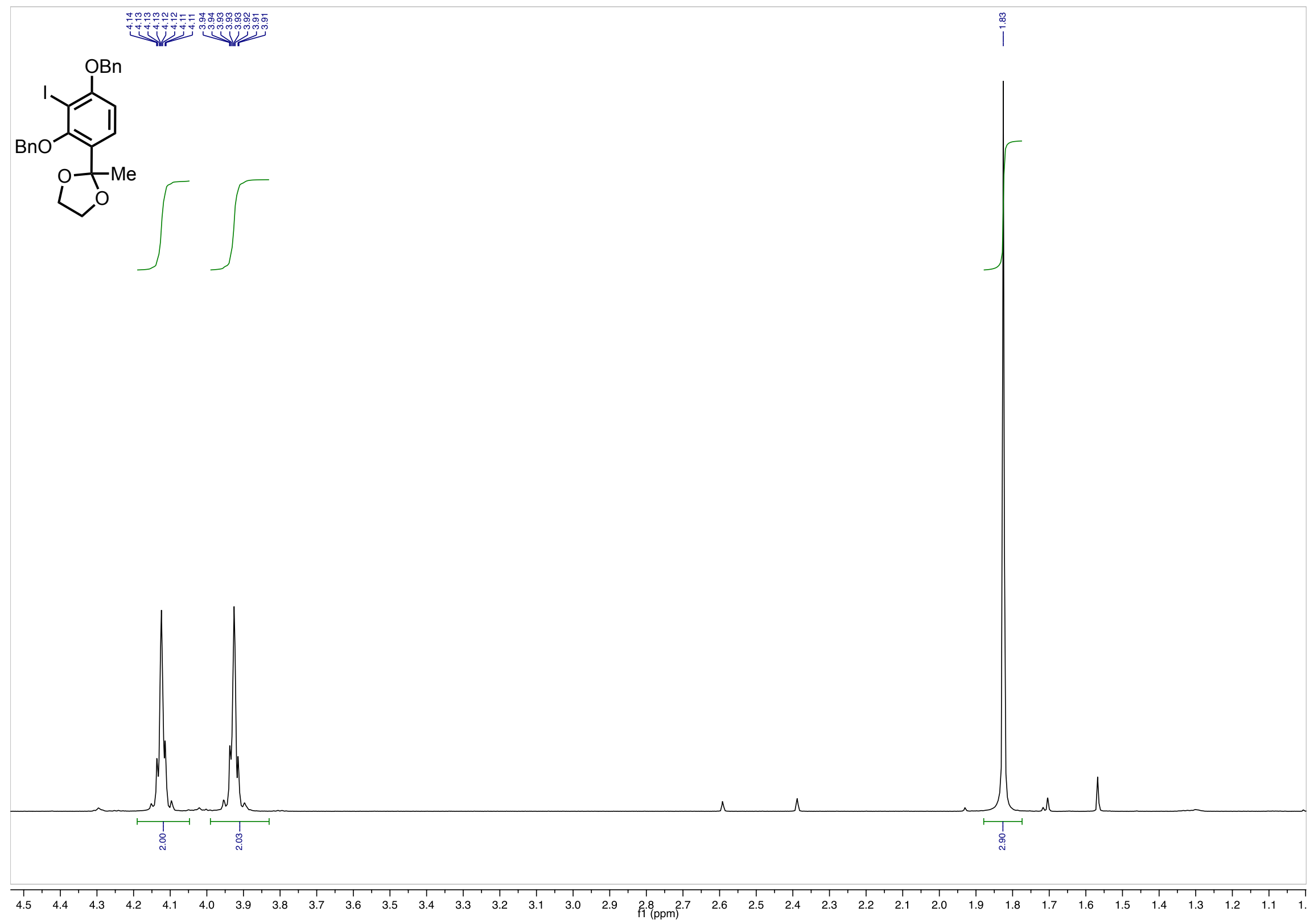

Figure S13. ${ }^{1 \mathrm{H}}$ NMR (600 MHz, chloroform-d), 7 (inset). 


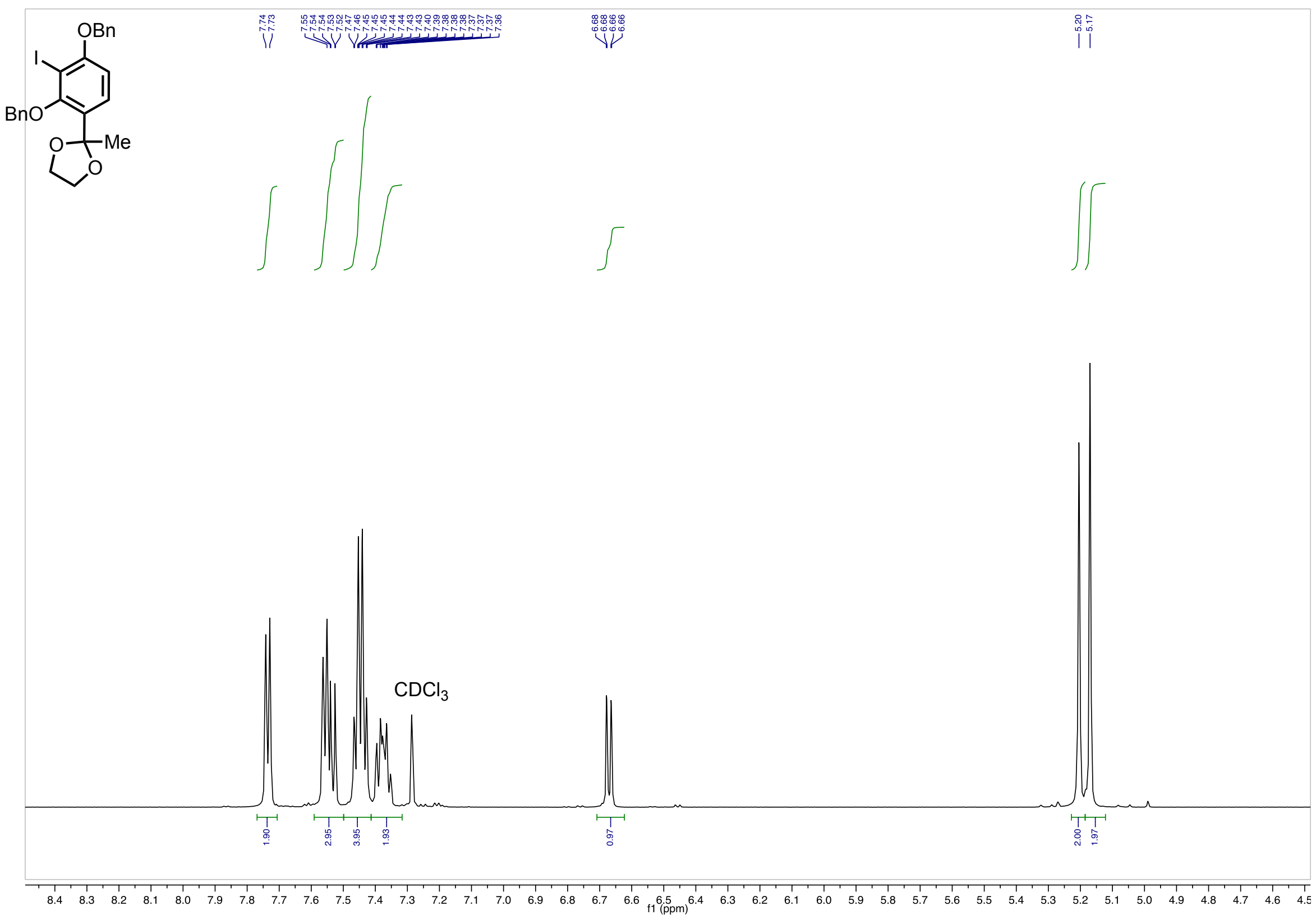

Figure S14. ${ }^{1} \mathrm{H}$ NMR (600 MHz, chloroform- $d$ ), 7 (inset). 

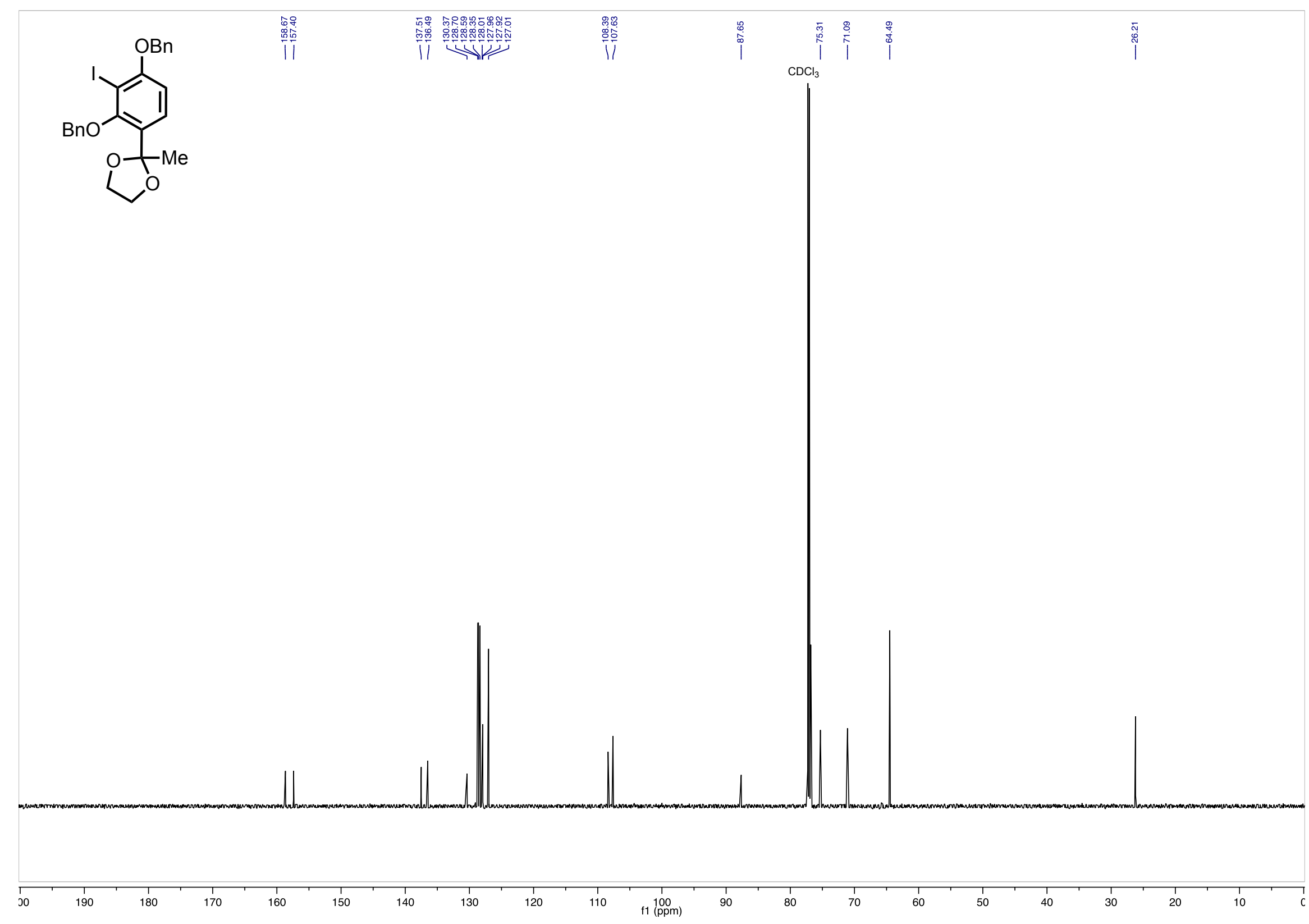

Figure S15. ${ }^{13} \mathrm{C}$ NMR (151 MHz, chloroform- $d$ ), 7. 

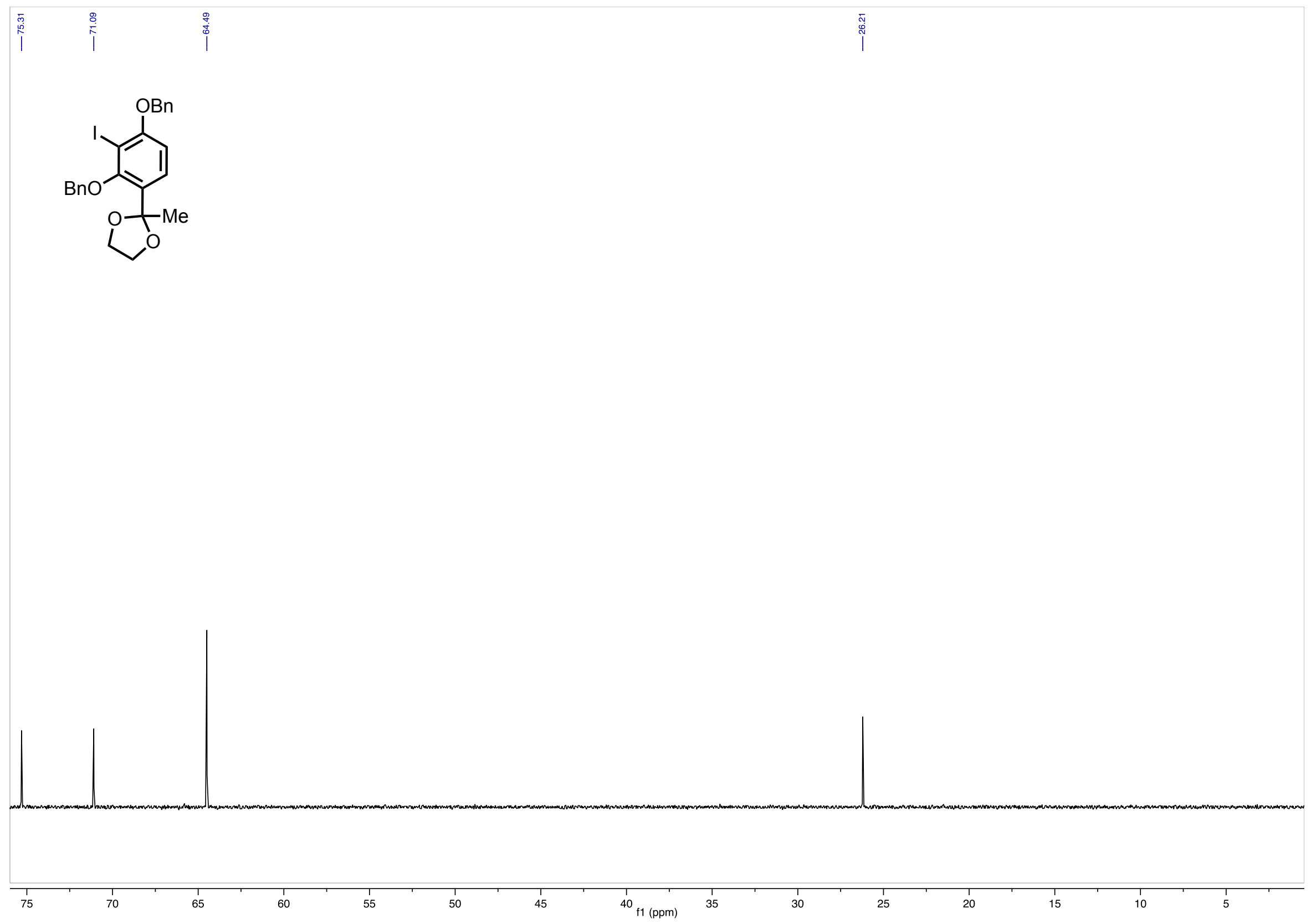

Figure S16. ${ }^{13} \mathrm{C}$ NMR (151 MHz, chloroform-d), 7 (inset). 

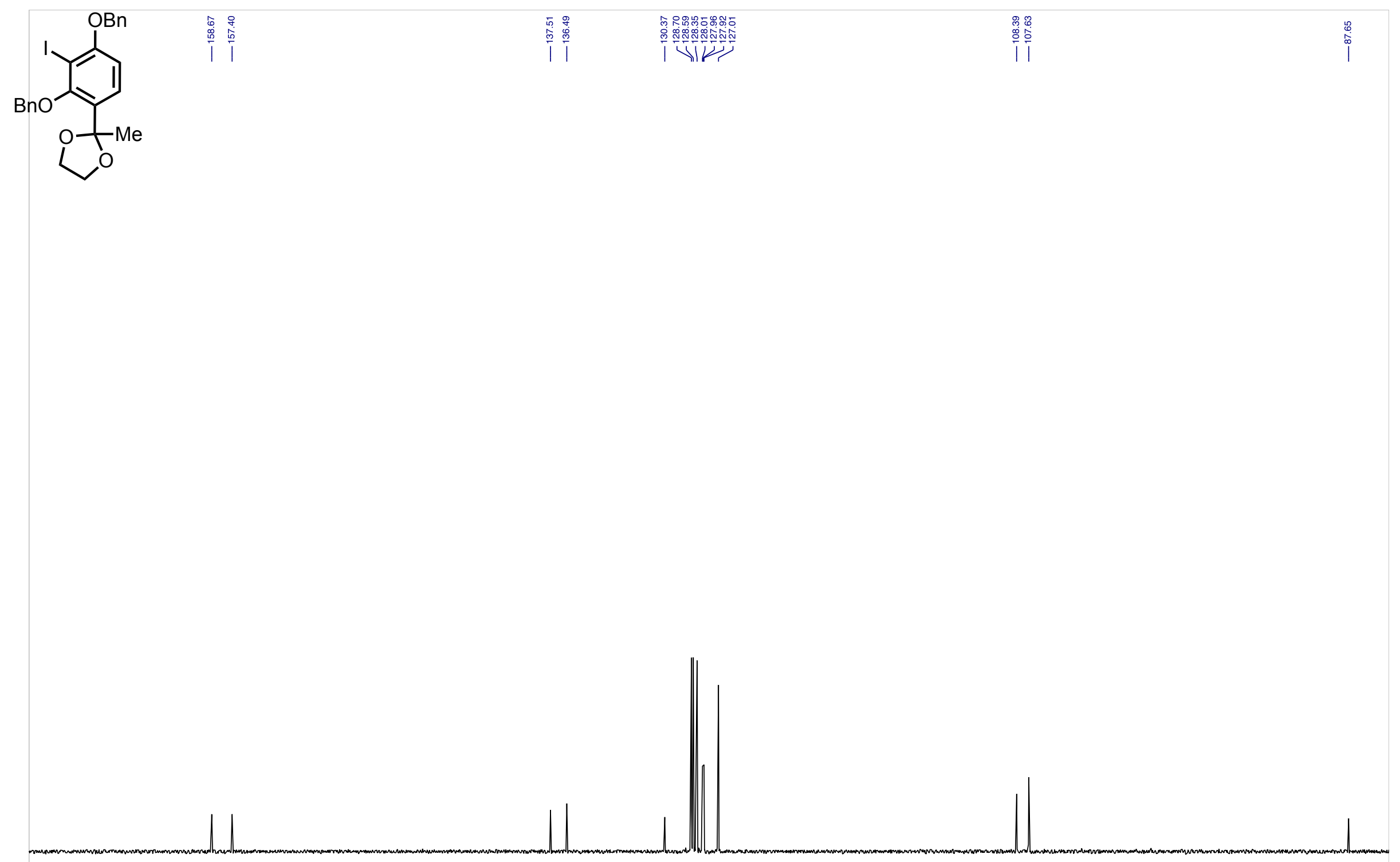

Figure S17. ${ }^{13} \mathrm{C}$ NMR (151 MHz, chloroform-d), 7 (inset). 


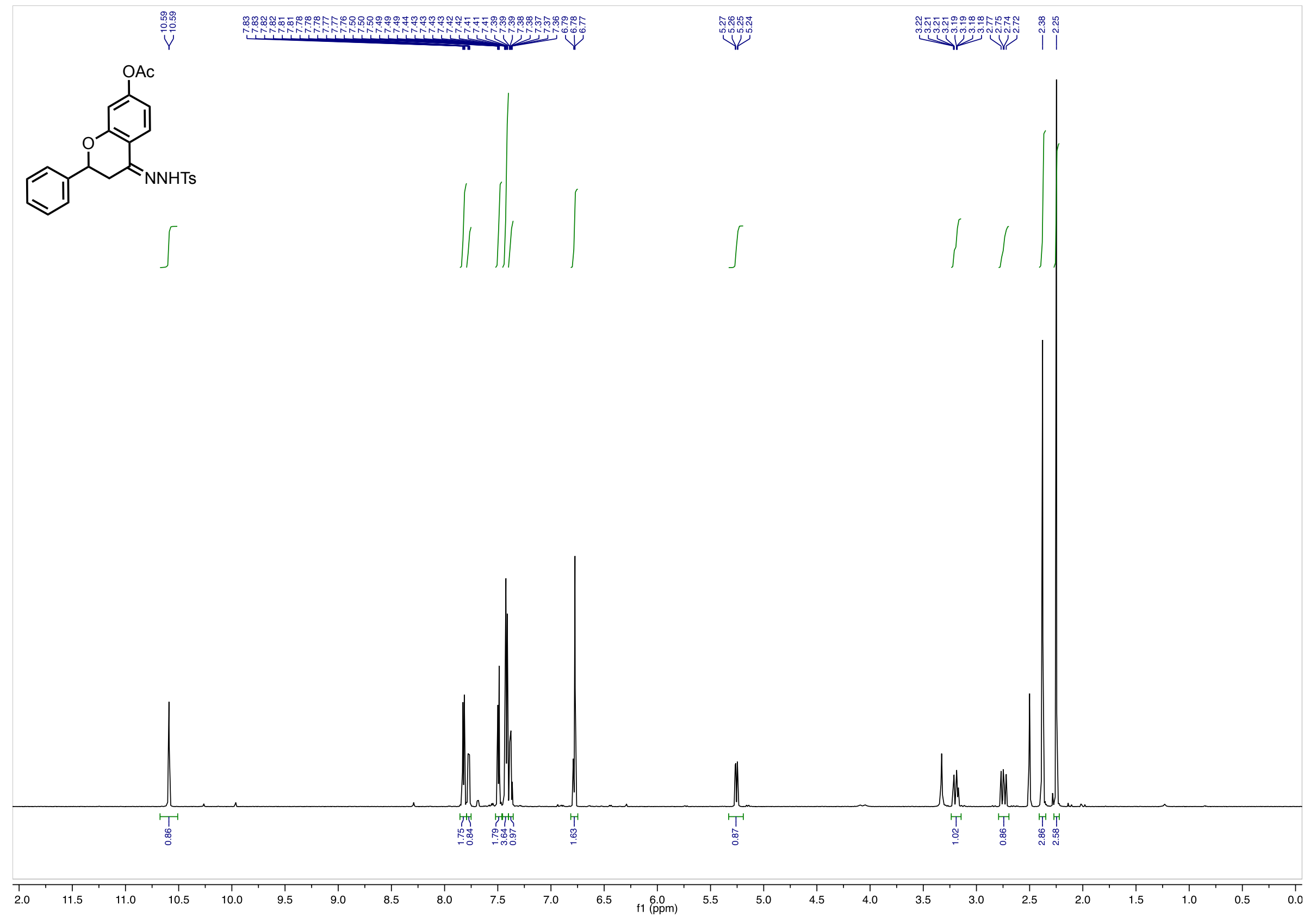

Figure S18. ${ }^{~} \mathrm{H}$ NMR (600 MHz, DMSO- $\left.d_{6}\right), 8$. 

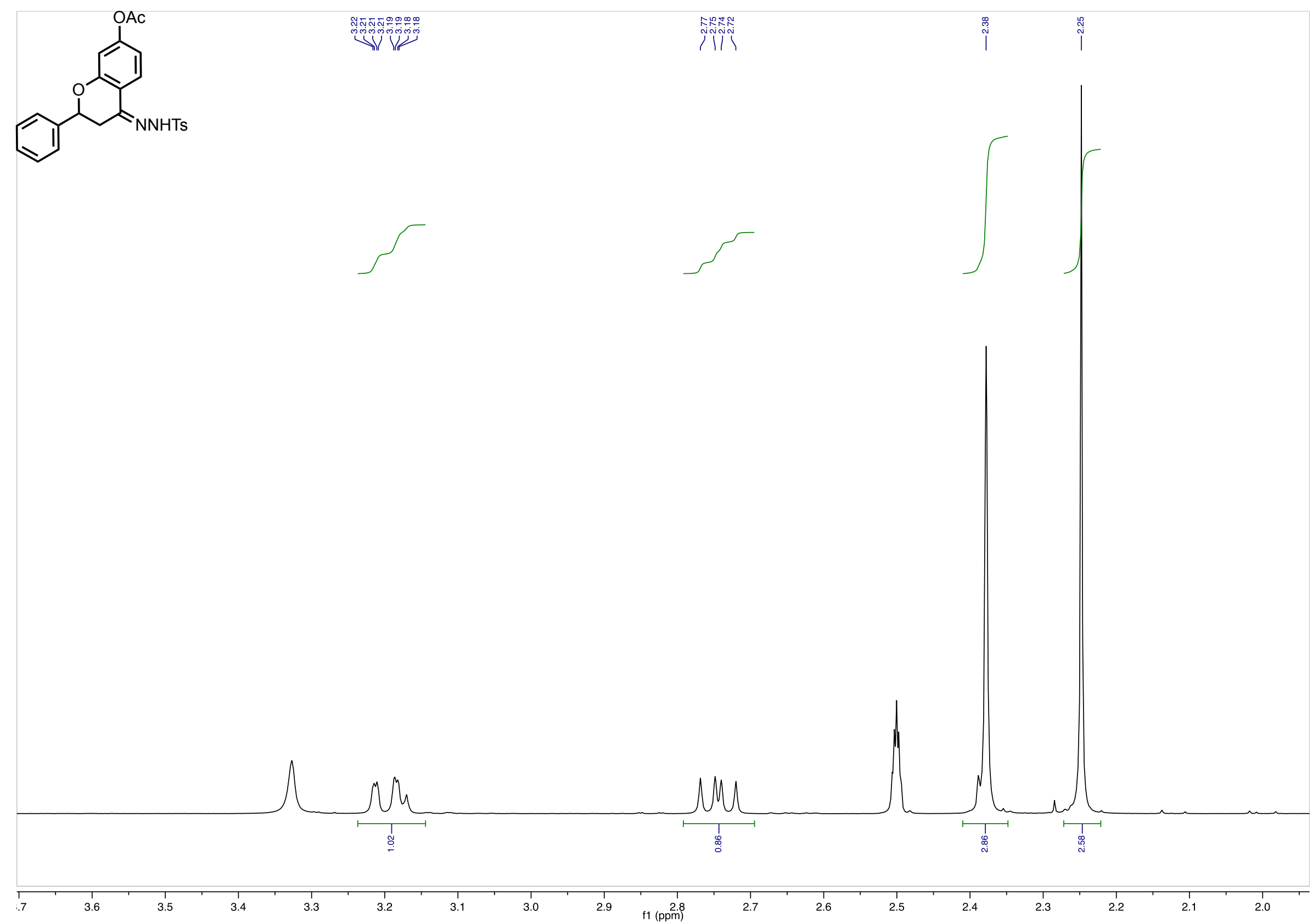

Figure S19. ${ }^{1} \mathrm{H}$ NMR (600 MHz, DMSO- $\left.d_{6}\right), 8$ (inset). 

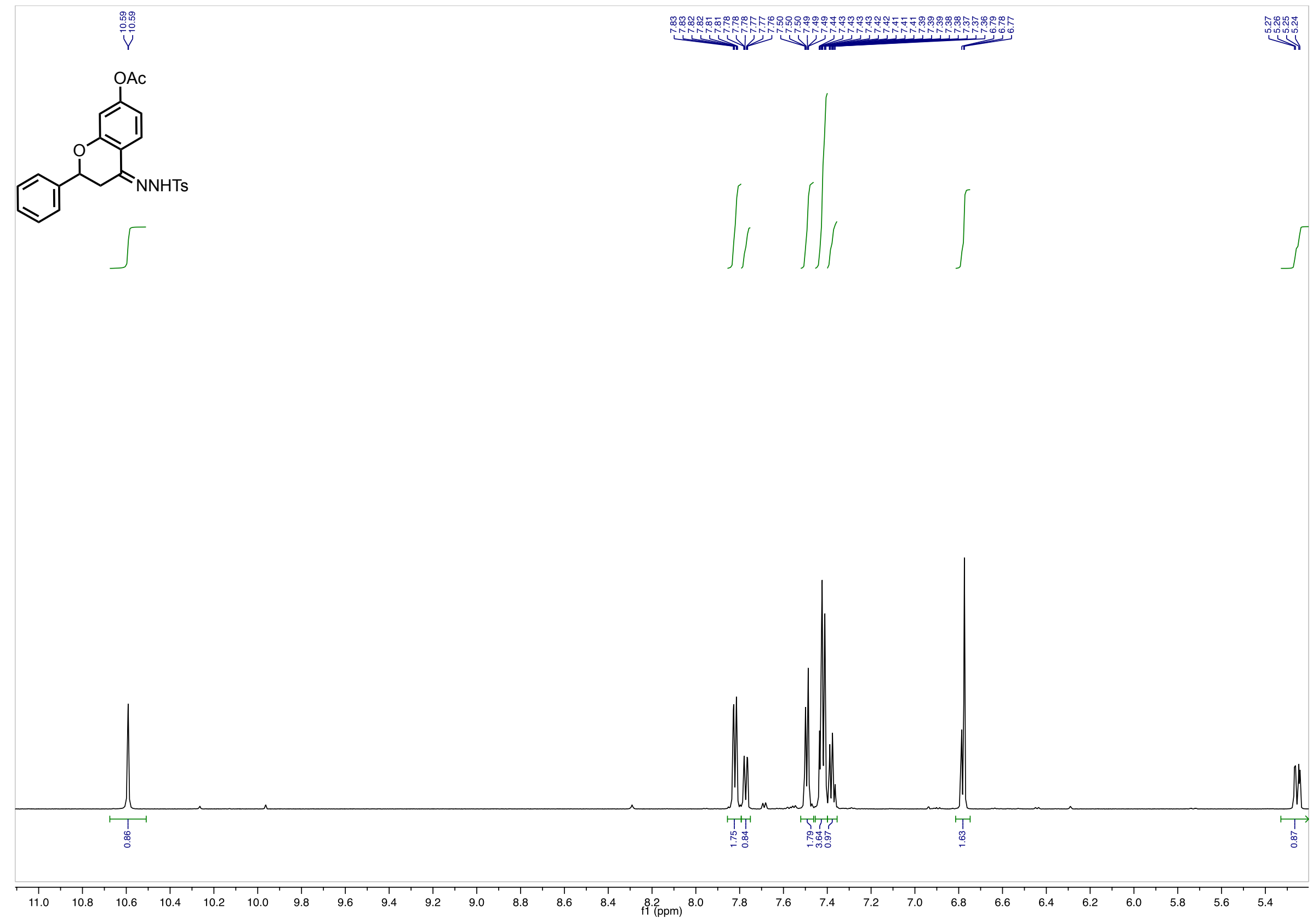

Figure S20. ${ }^{1} \mathrm{H}$ NMR (600 MHz, DMSO-d6), 8 (inset). 

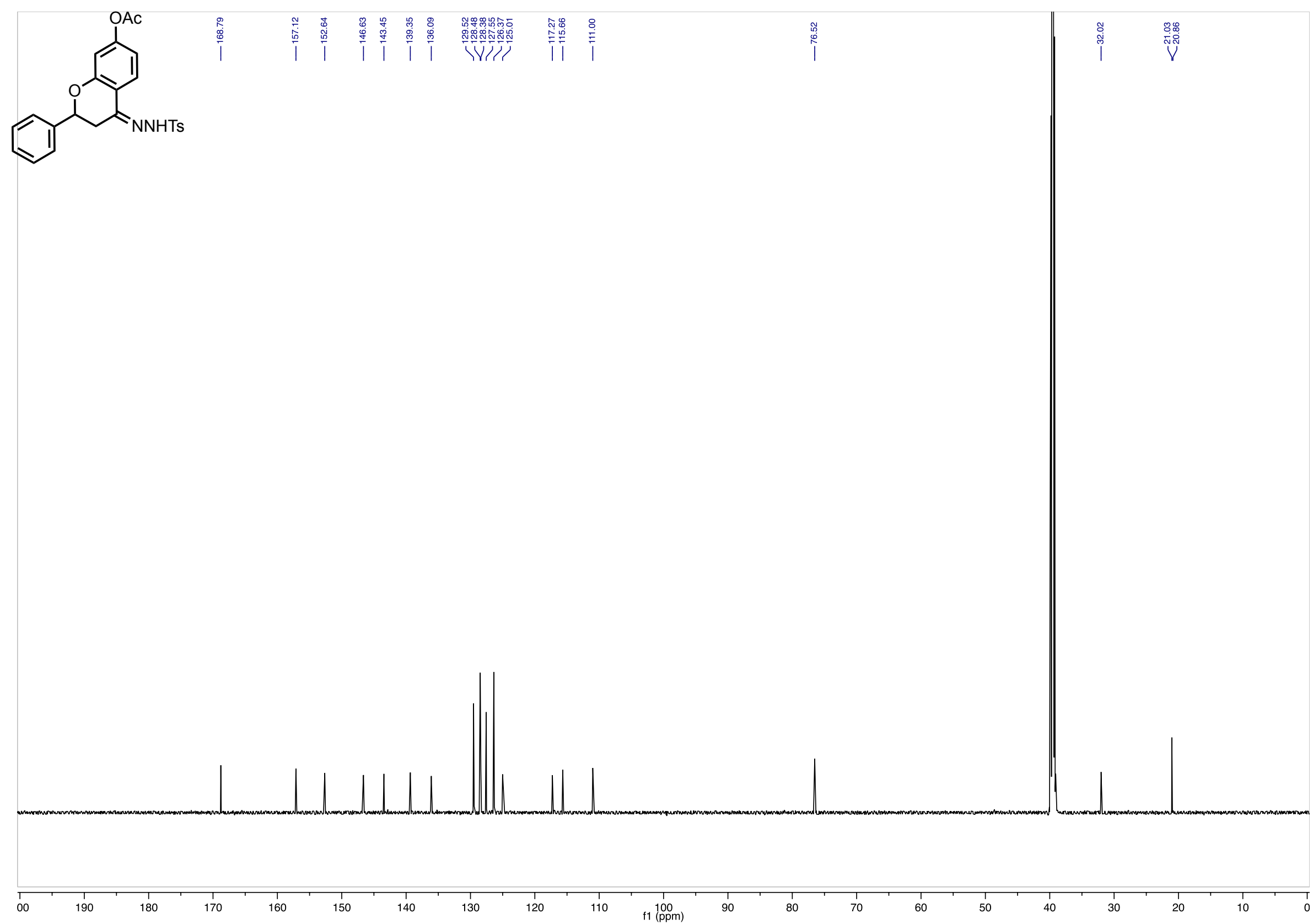

Figure S21. ${ }^{13} \mathrm{C}$ NMR (151 MHz, DMSO-d6), 8. 


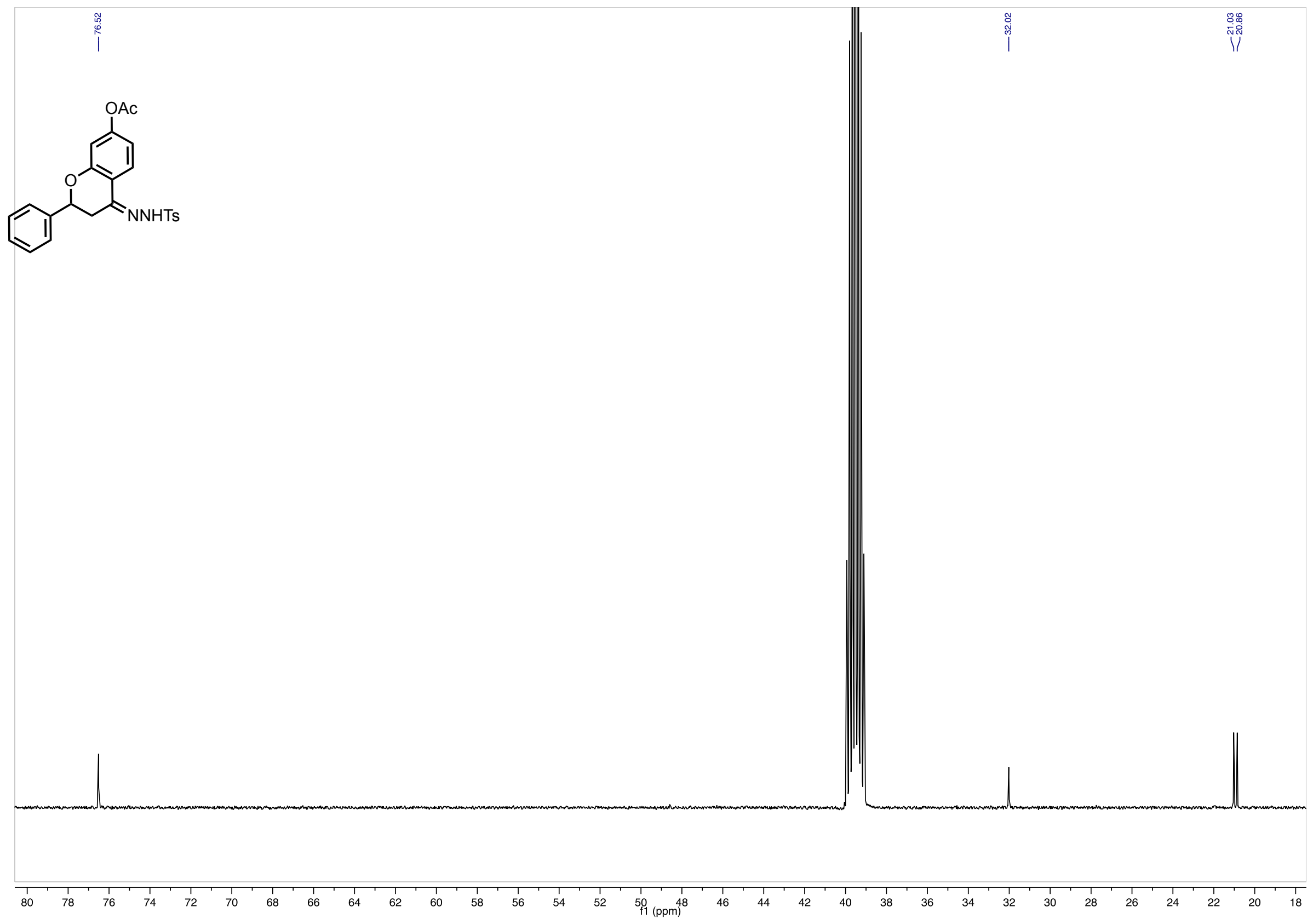

Figure S22. ${ }^{13} \mathrm{C}$ NMR (151 MHz, DMSO- $\left.d_{6}\right), 8$ (inset).

S78 

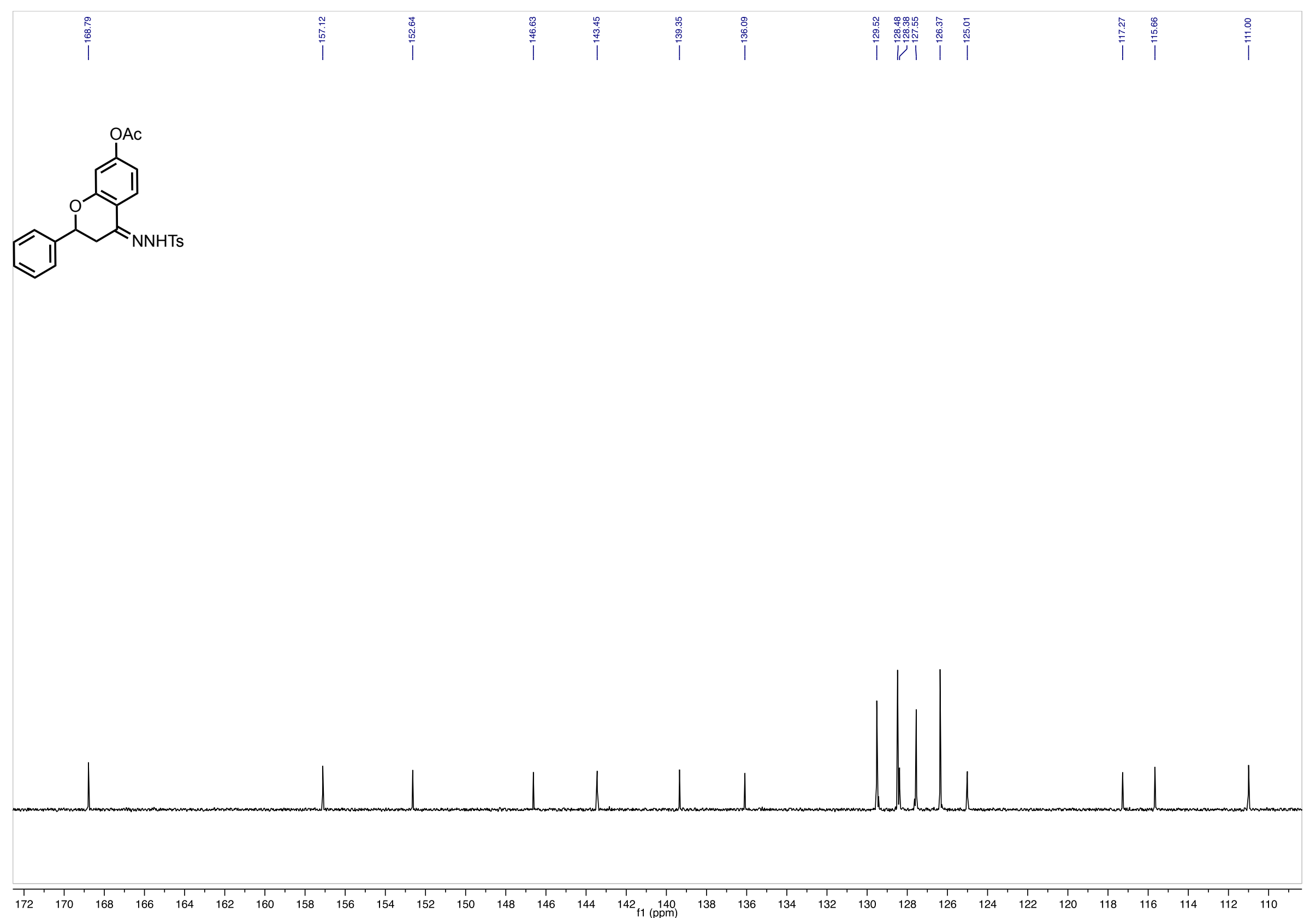

Figure $S 23 .{ }^{13} \mathrm{C}$ NMR (151 MHz, DMSO- $\left.d_{6}\right), 8$ (inset). 

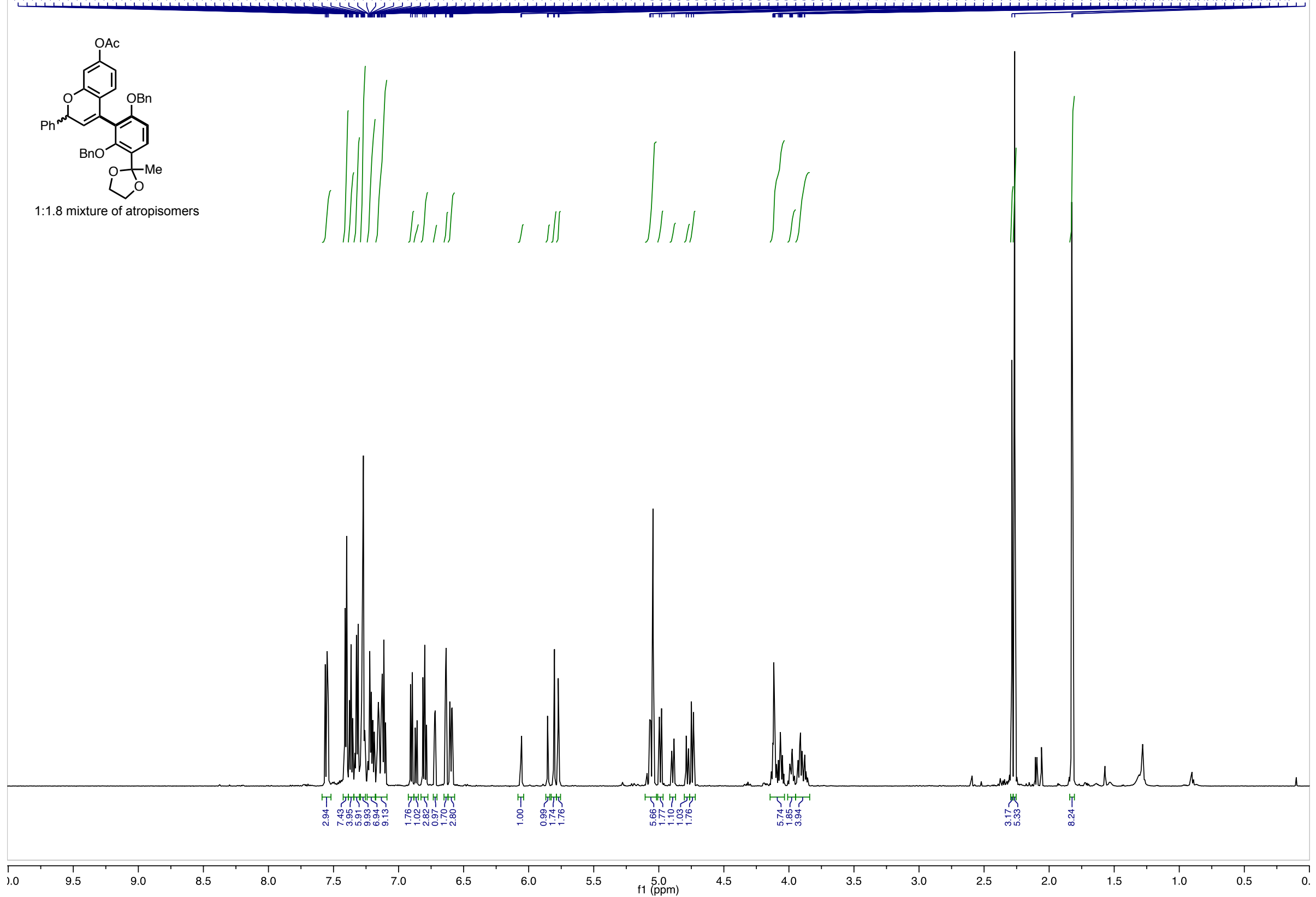

Figure S24. ${ }^{1} \mathrm{H}$ NMR (600 MHz, chloroform- $d$ ), 9. 

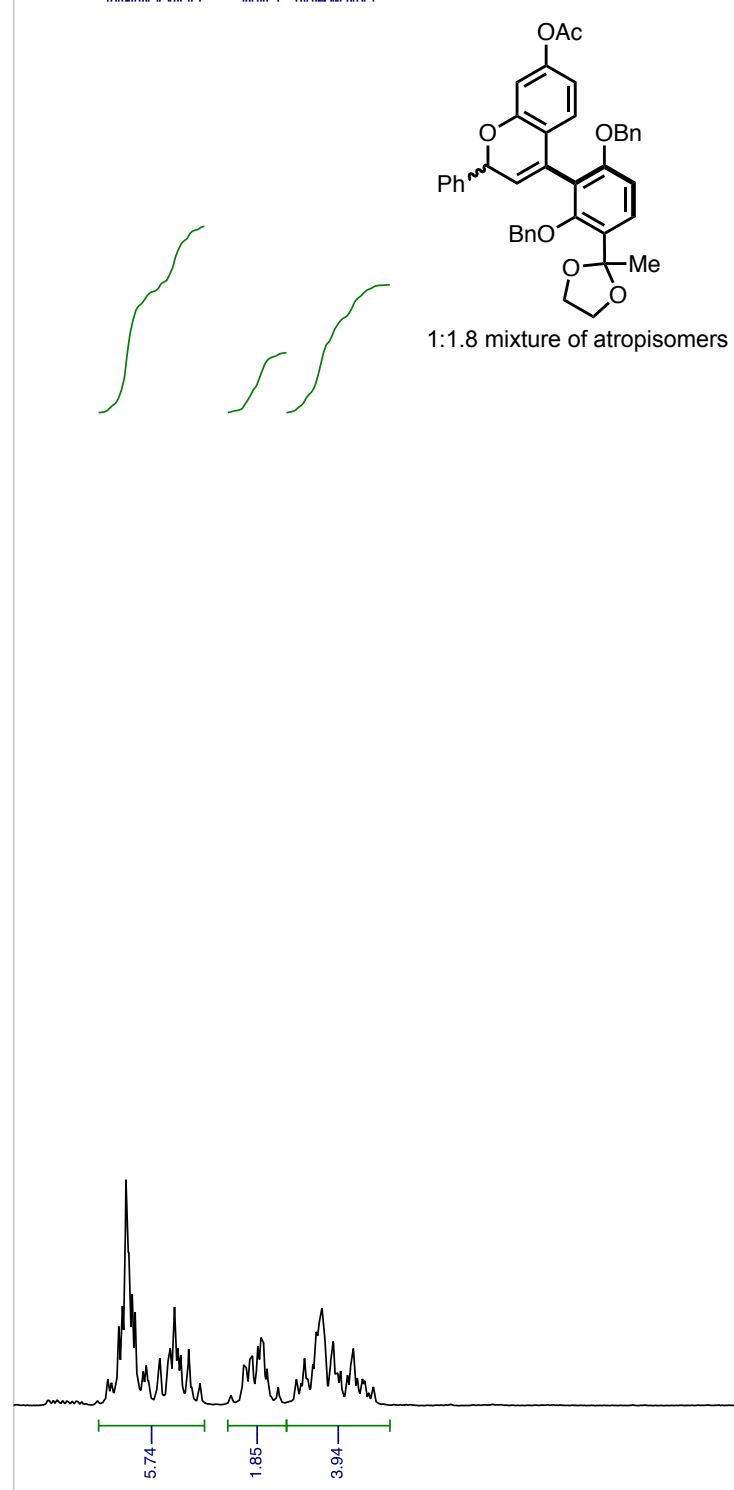

Figure S25. ${ }^{1 \mathrm{H}} \mathrm{NMR}(600 \mathrm{MHz}$, chloroform- $d$ ), 9 (inset). 


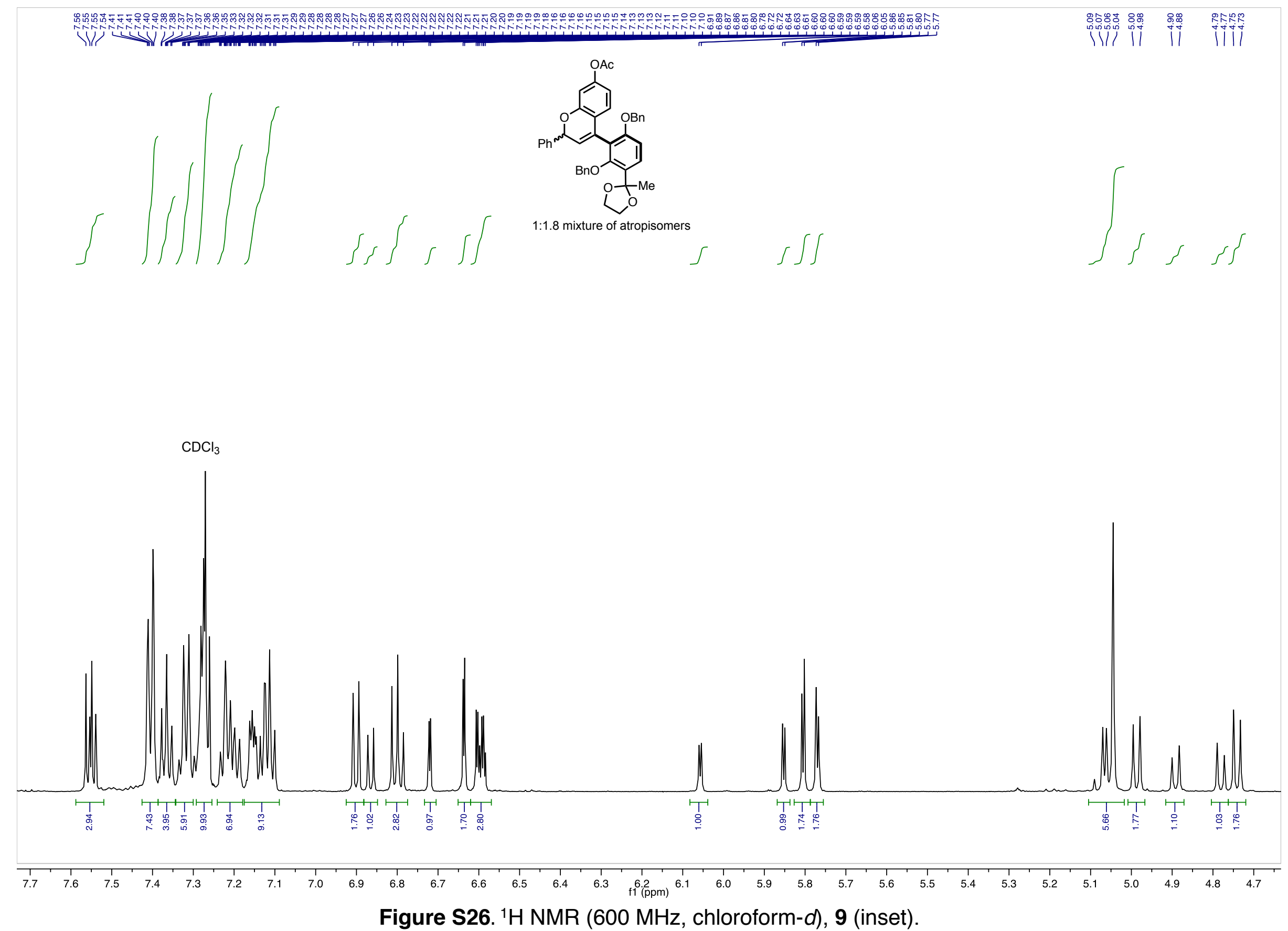



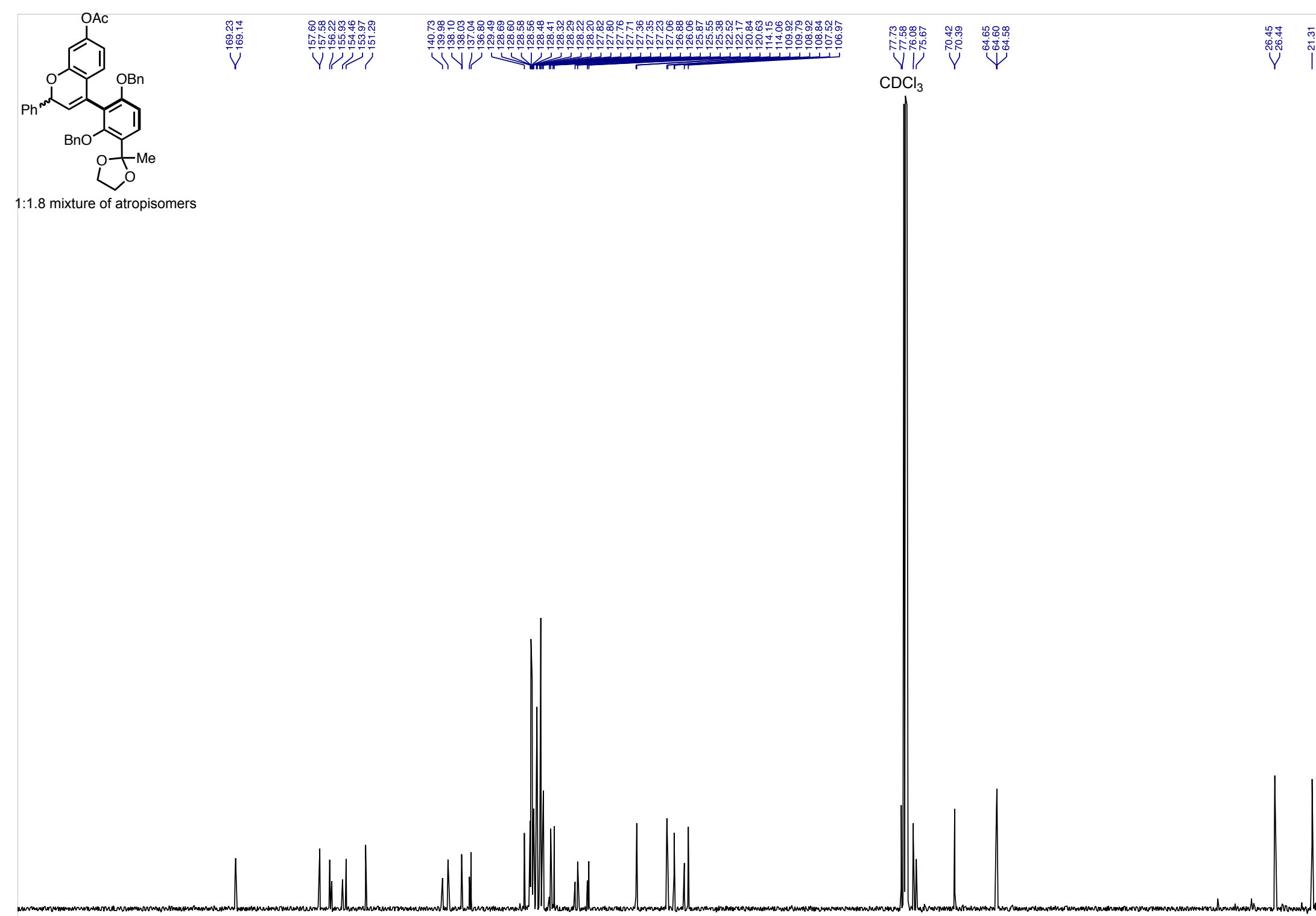

Figure S27. ${ }^{13} \mathrm{C}$ NMR (151 MHz, chloroform- $d$ ), 9. 


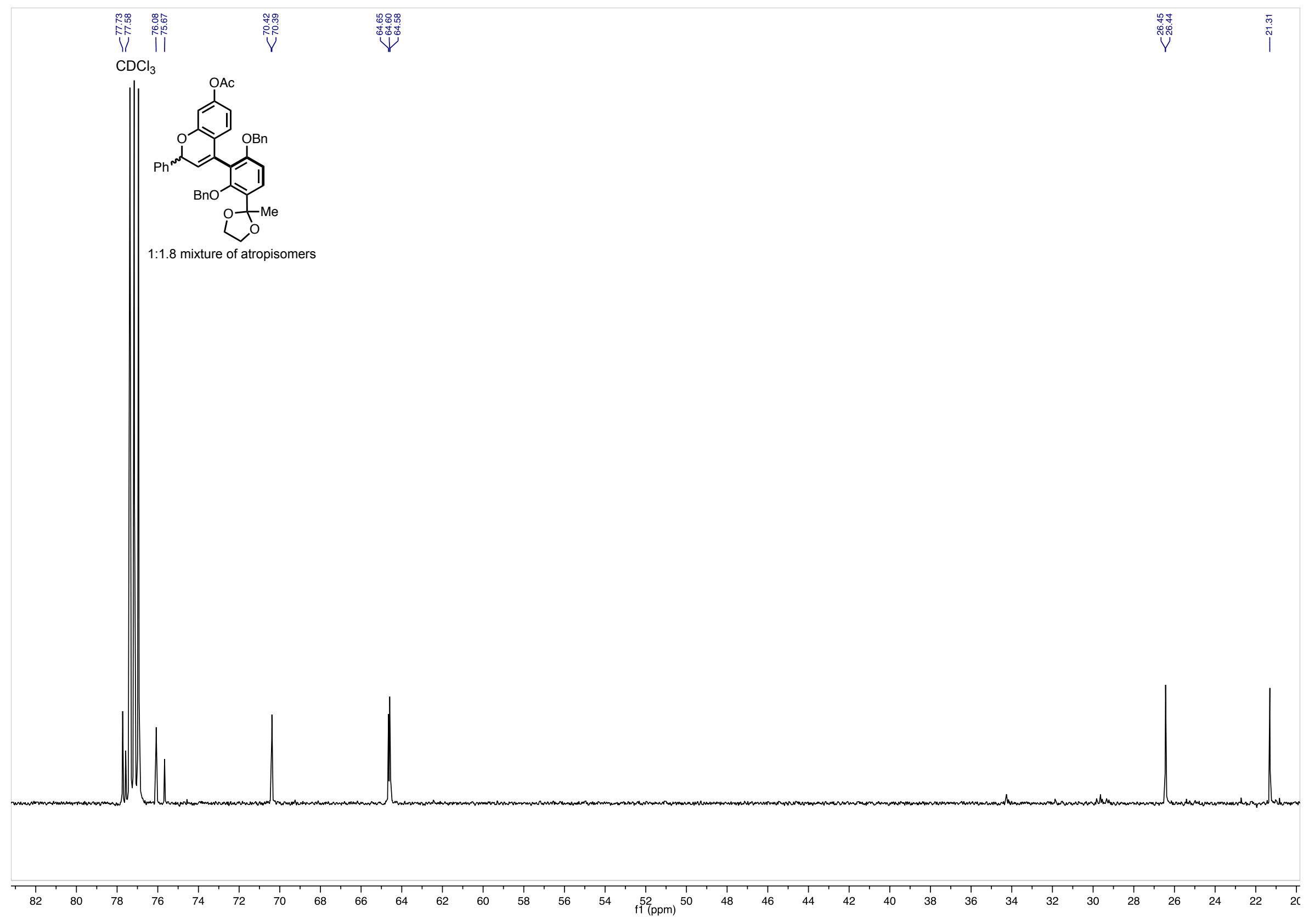

Figure S28. ${ }^{13} \mathrm{C}$ NMR (151 MHz, chloroform- $d$ ), 9 (inset).

S84 

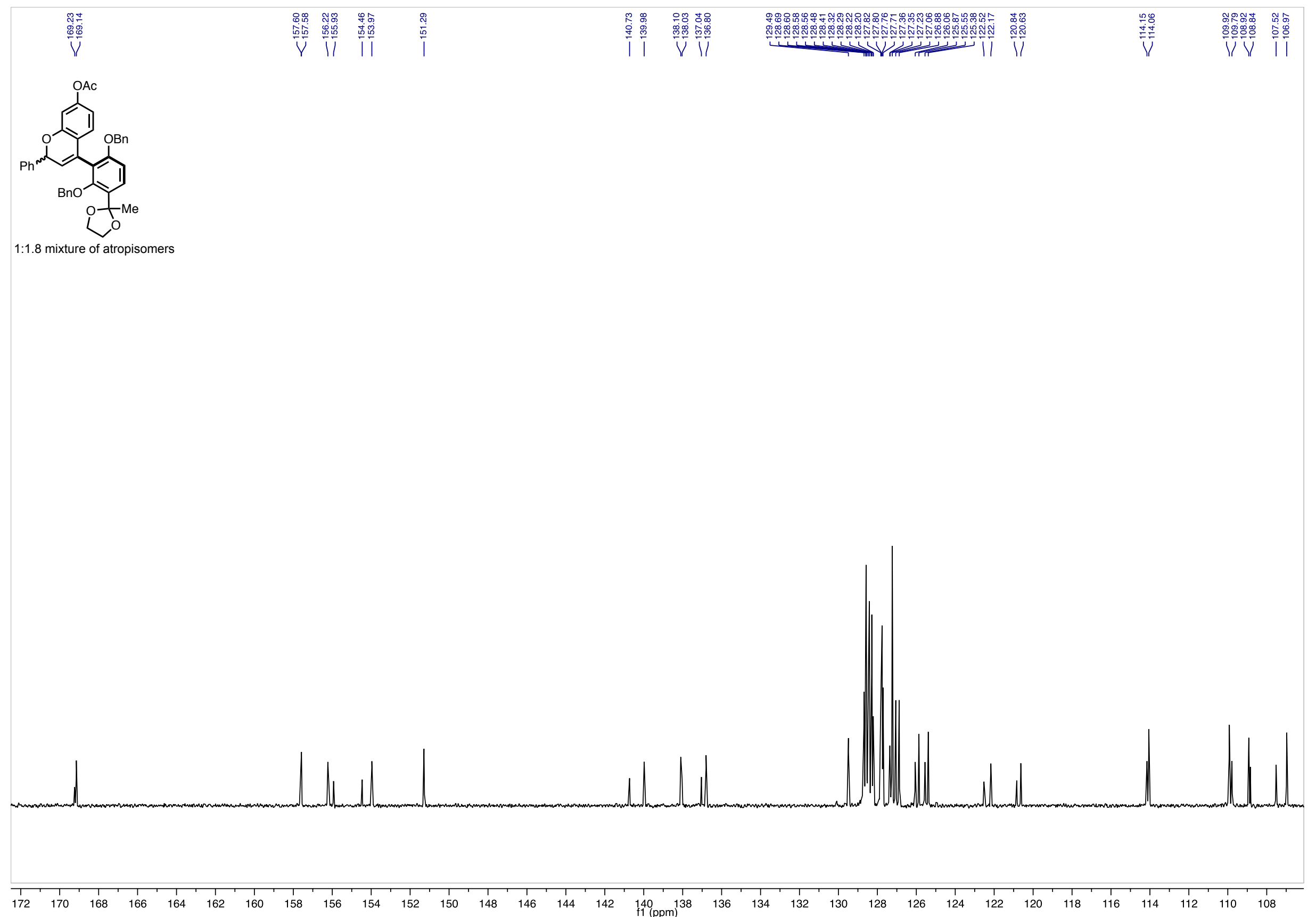

Figure S29. ${ }^{13} \mathrm{C}$ NMR (151 MHz, chloroform-d), 9 (inset). 


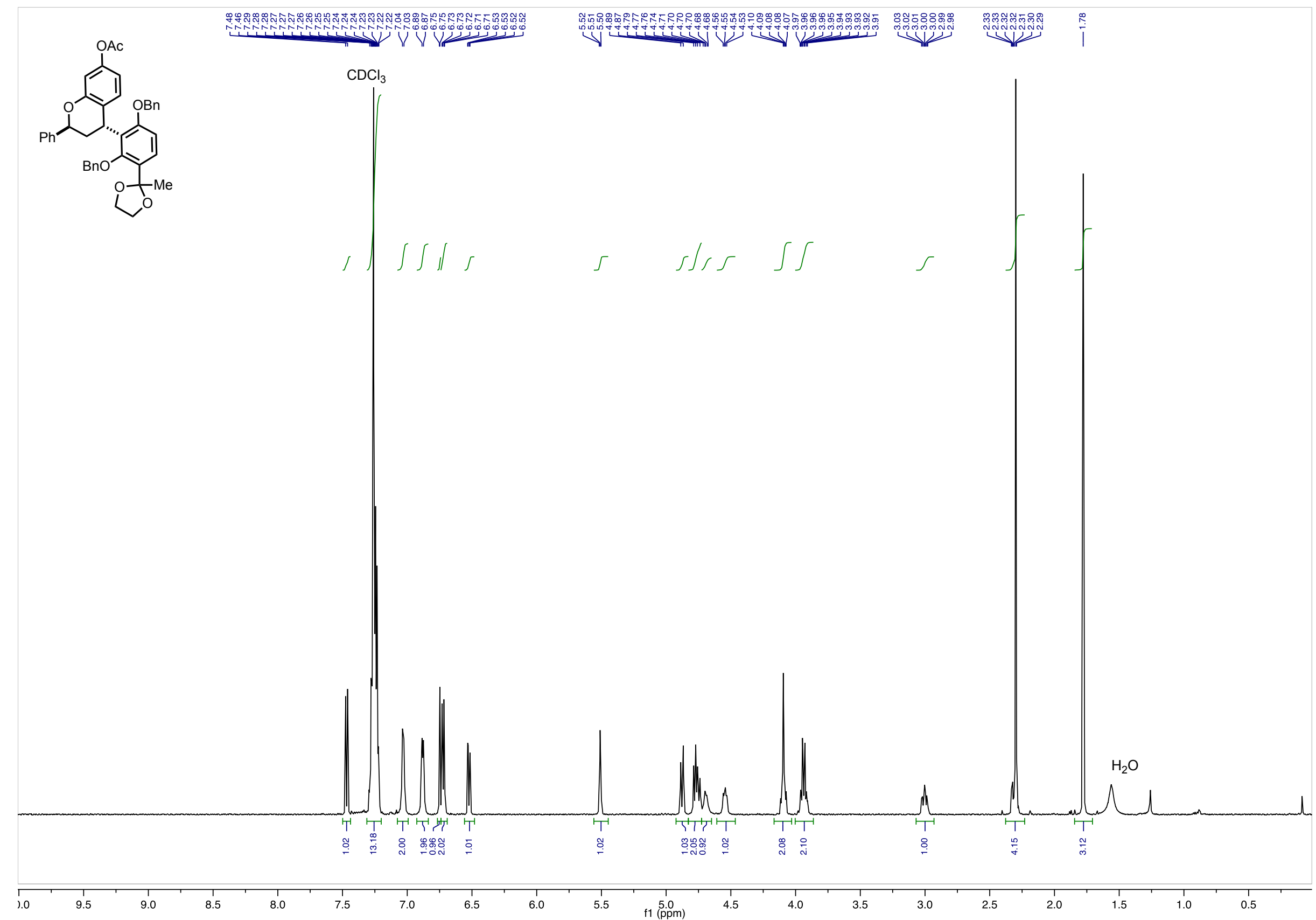

Figure S30. ${ }^{1} \mathrm{H}$ NMR (600 MHz, chloroform- $d$ ), 10a. 


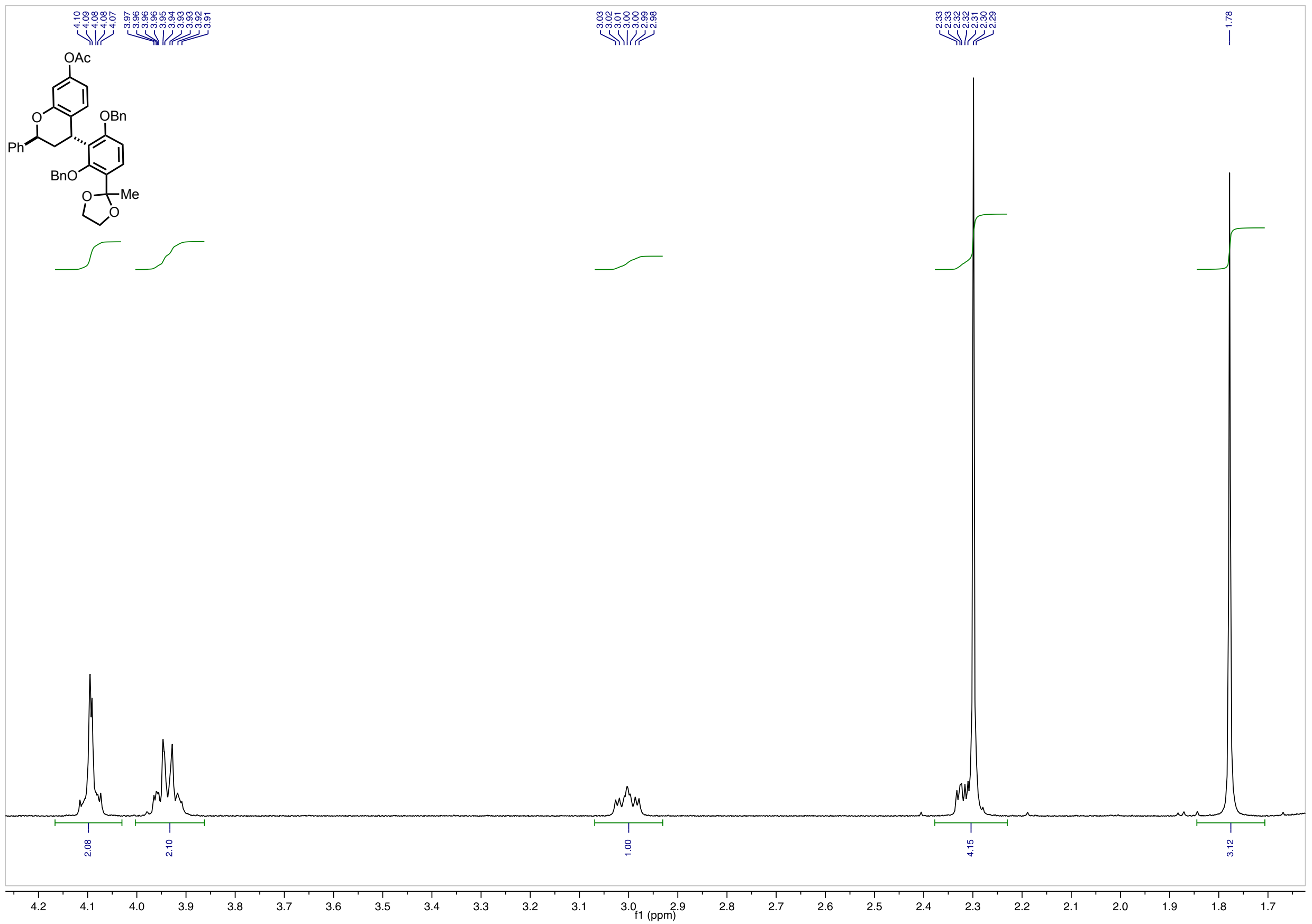

Figure S31. ${ }^{1} \mathrm{H}$ NMR (600 MHz, chloroform- $d$ ), 10a (inset). 


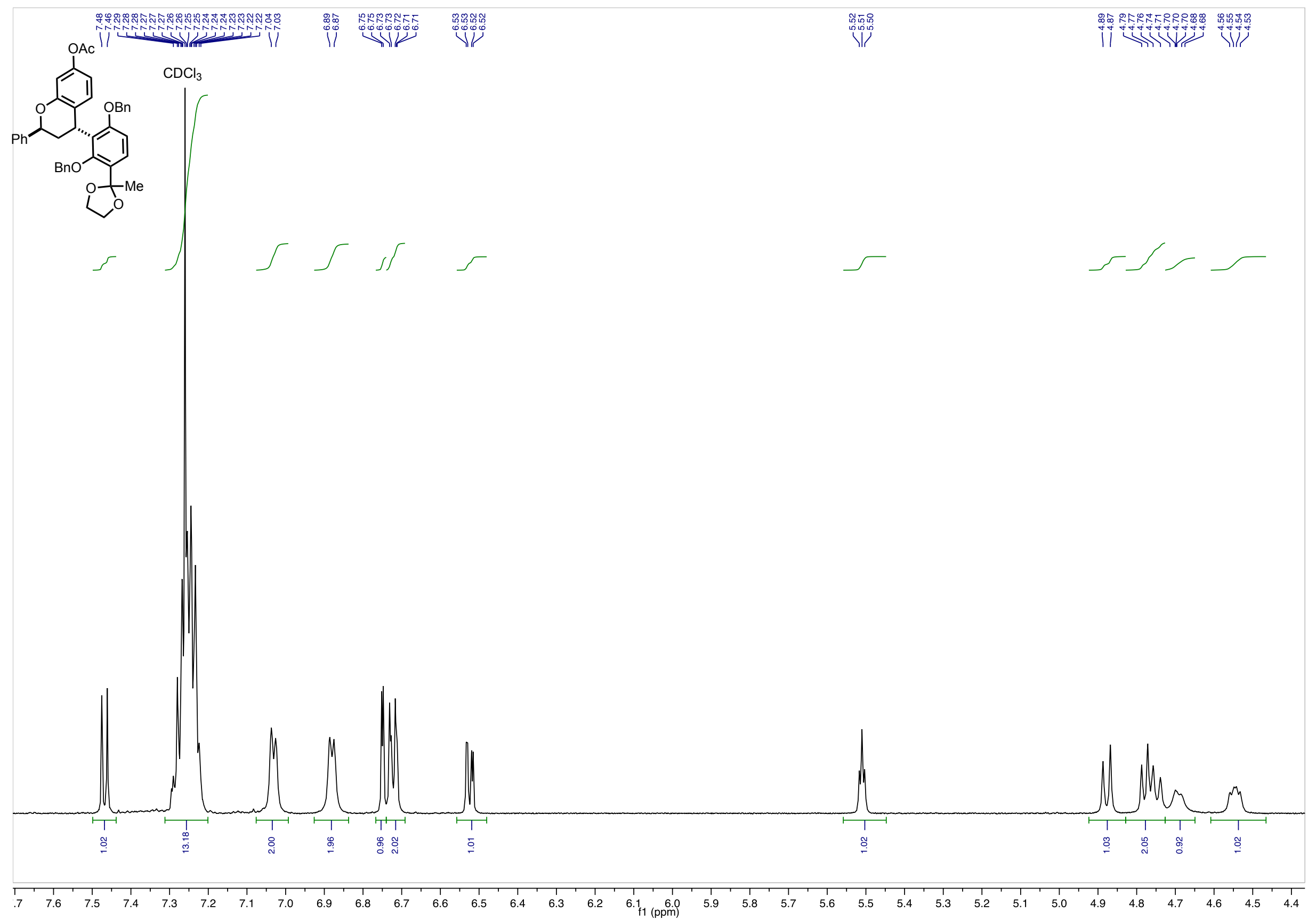

Figure S32. ${ }^{1} \mathrm{H}$ NMR (600 MHz, chloroform- $d$ ), 10a (inset). 


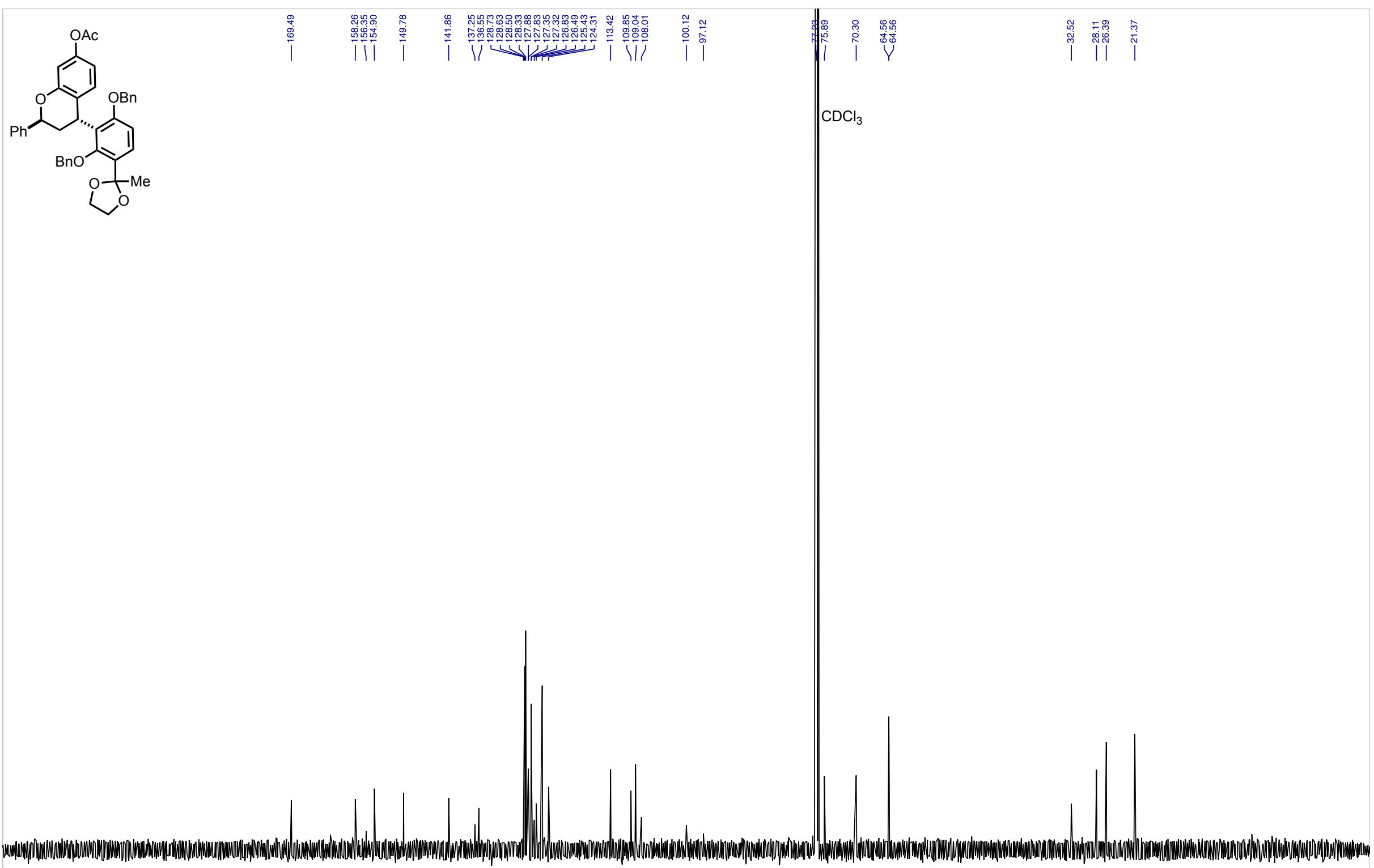

20

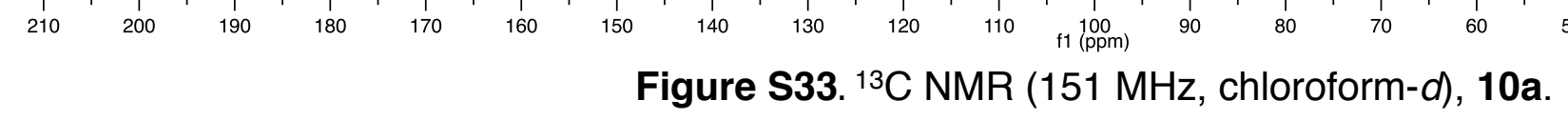




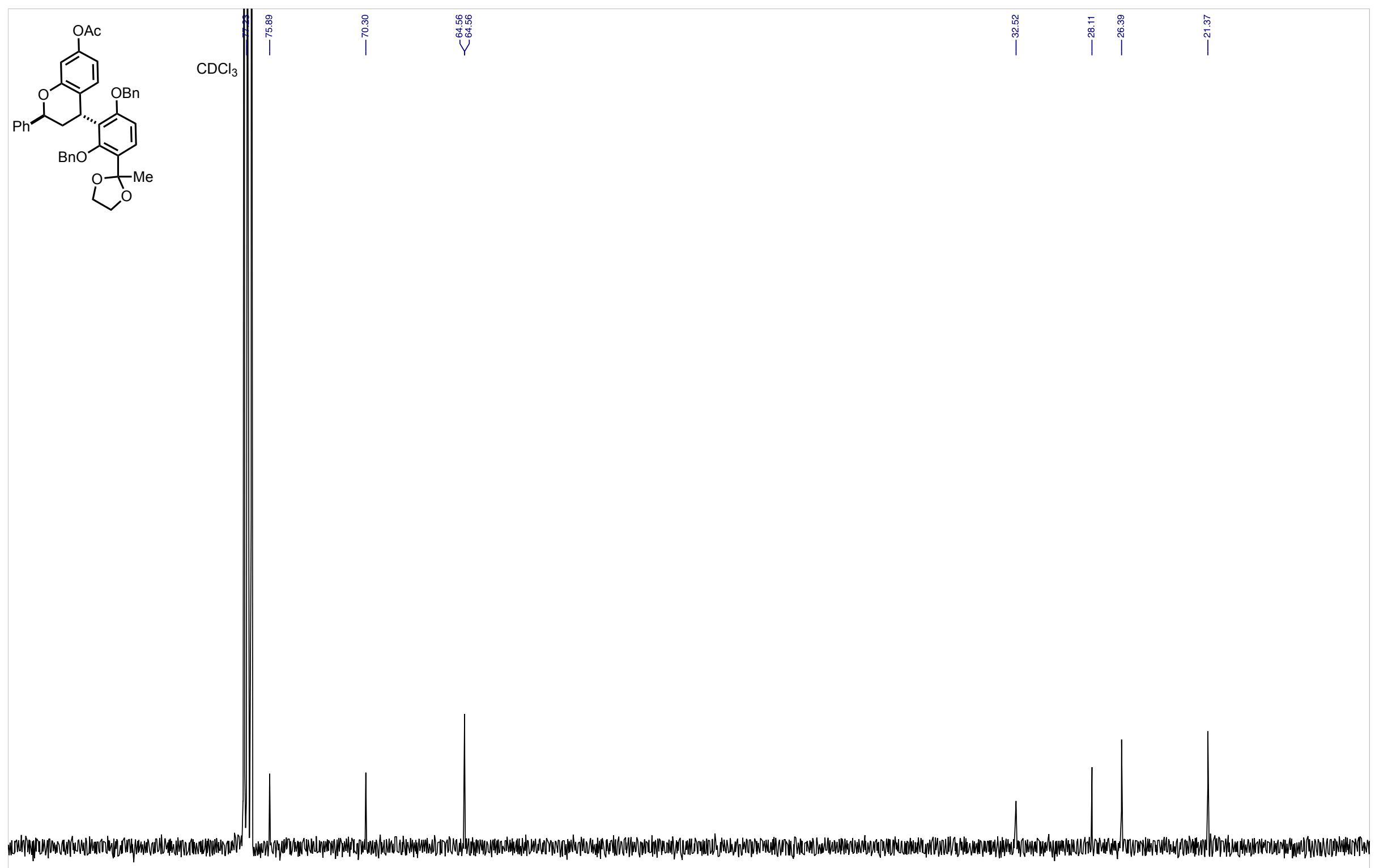

Figure S34. ${ }^{13} \mathrm{C}$ NMR (151 MHz, chloroform-d), 10a (inset). 


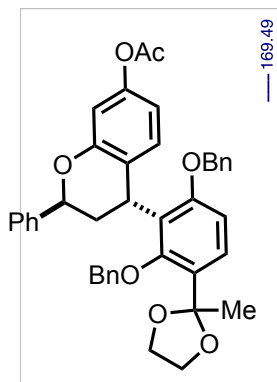

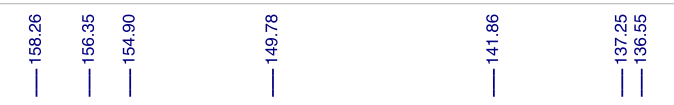
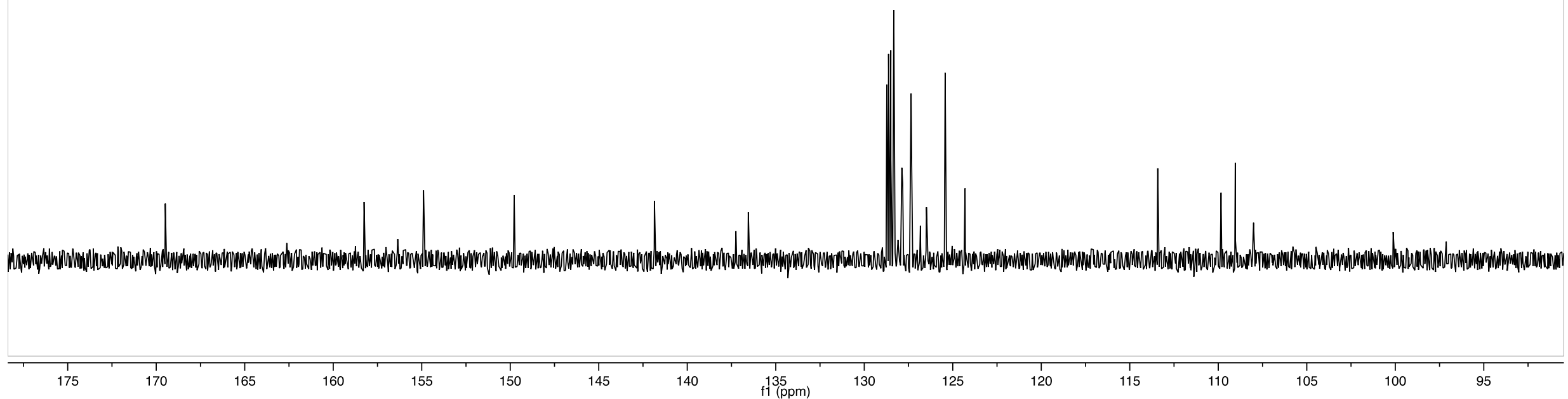

Figure S35. ${ }^{13} \mathrm{C}$ NMR (151 MHz, chloroform- $d$ ), 10a (inset). 


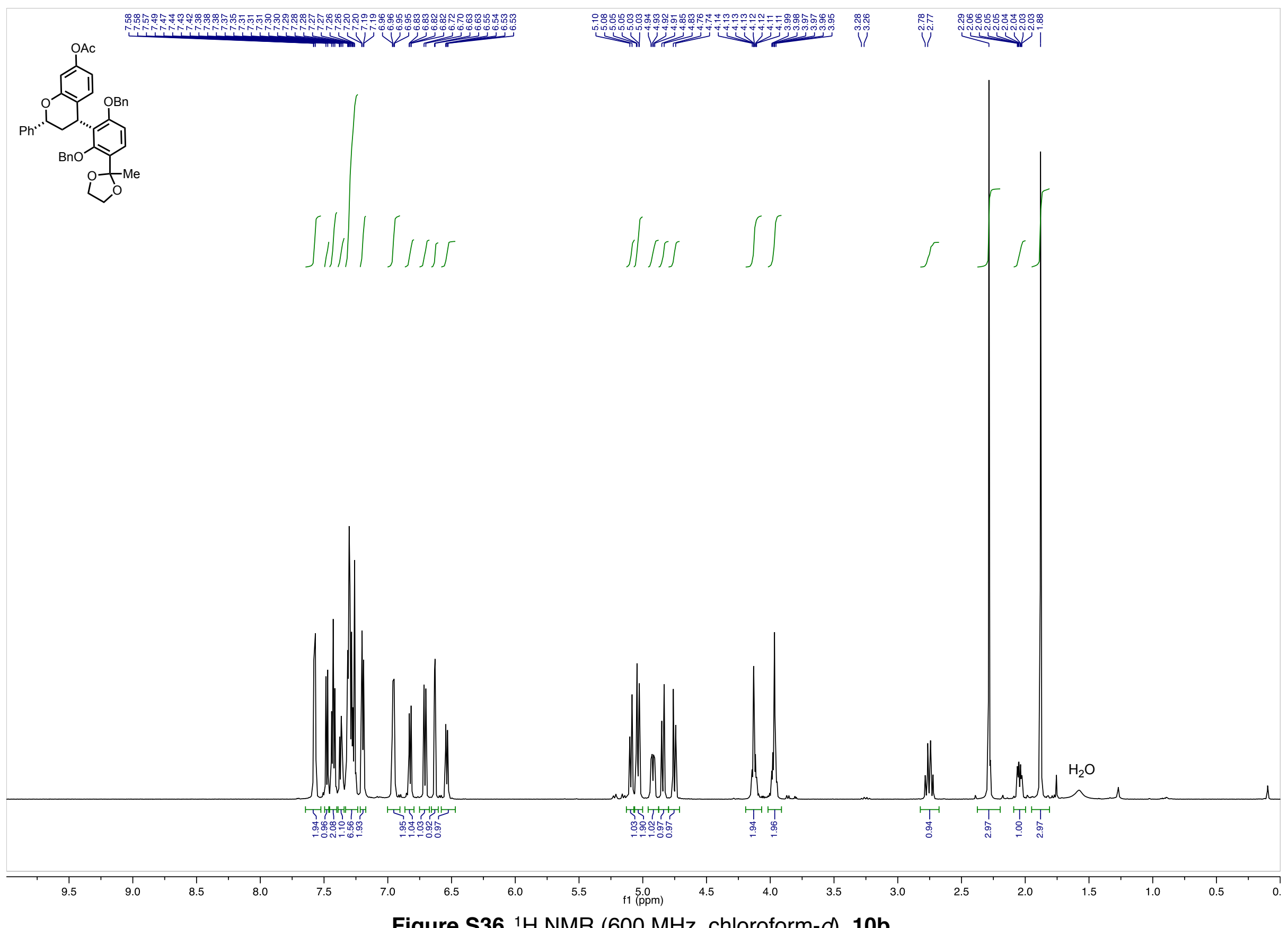

Figure S36. ${ }^{1} \mathrm{H}$ NMR (600 MHz, chloroform- $d$ ), $10 \mathrm{~b}$. 


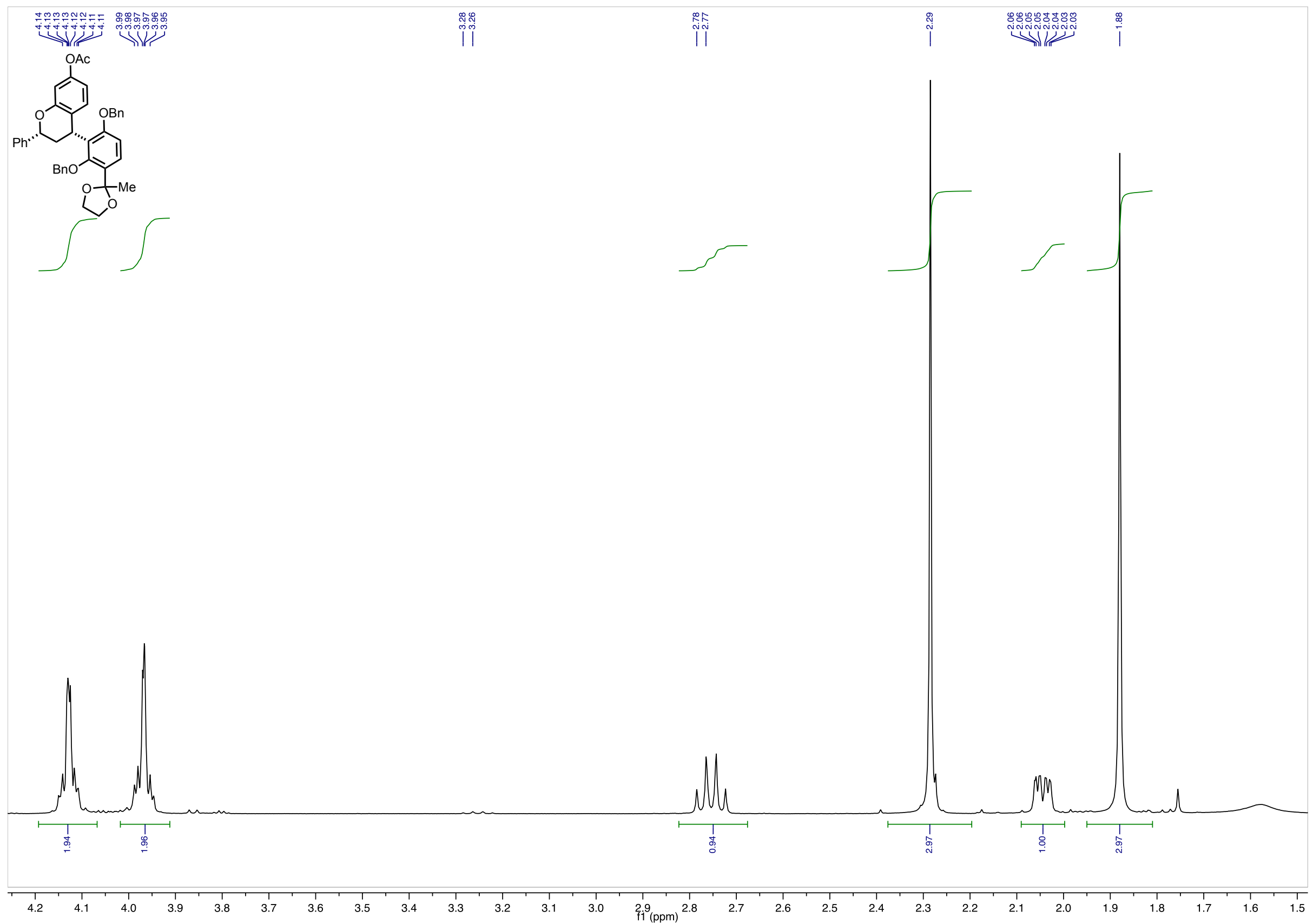

Figure S37. ${ }^{1} \mathrm{H}$ NMR $(600 \mathrm{MHz}$, chloroform- $d$ ), 10b (inset). 


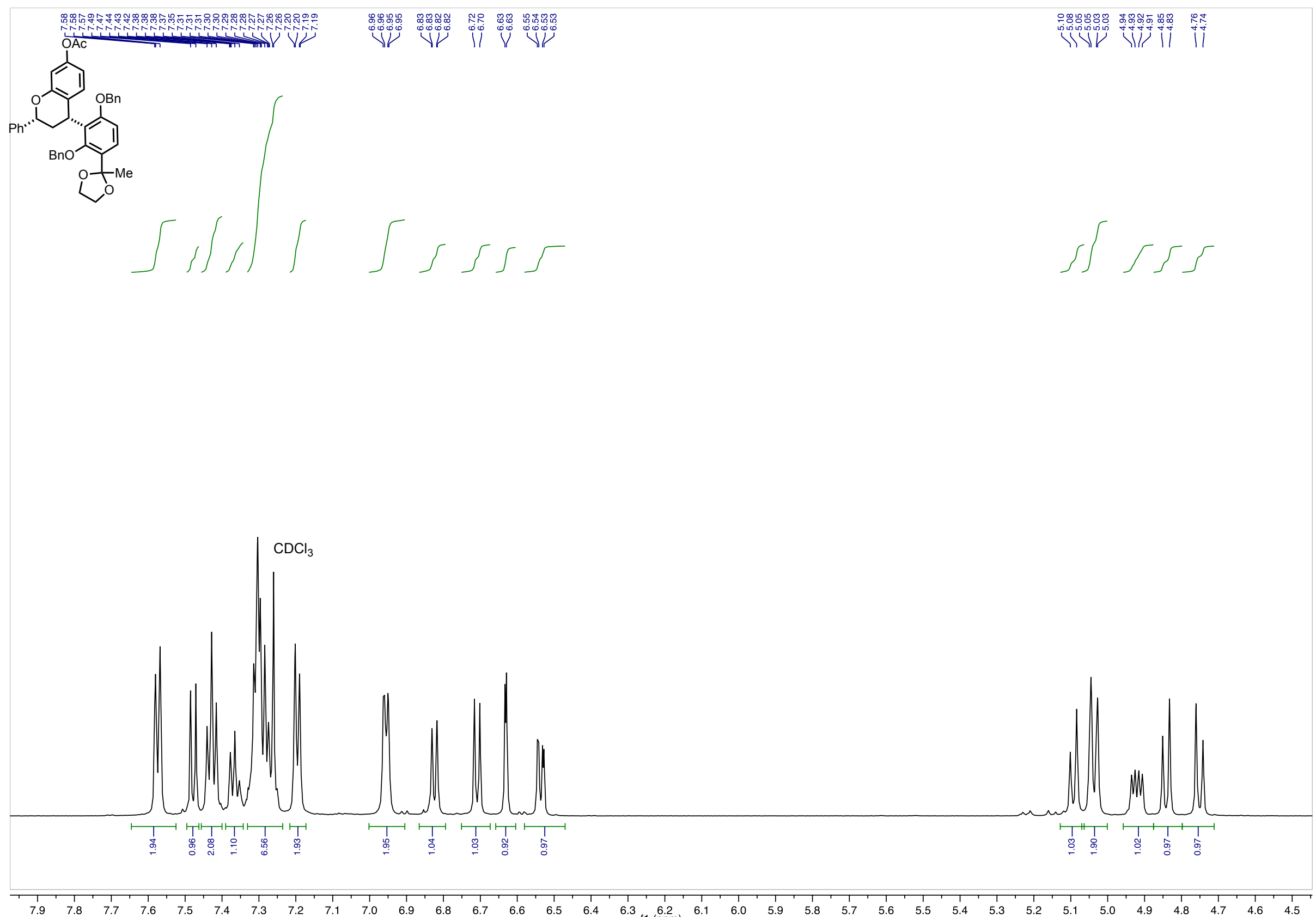

Figure S38. ${ }^{1 \mathrm{H}} \mathrm{NMR}(600 \mathrm{MHz}$, chloroform- $d$ ), 10b (inset). 

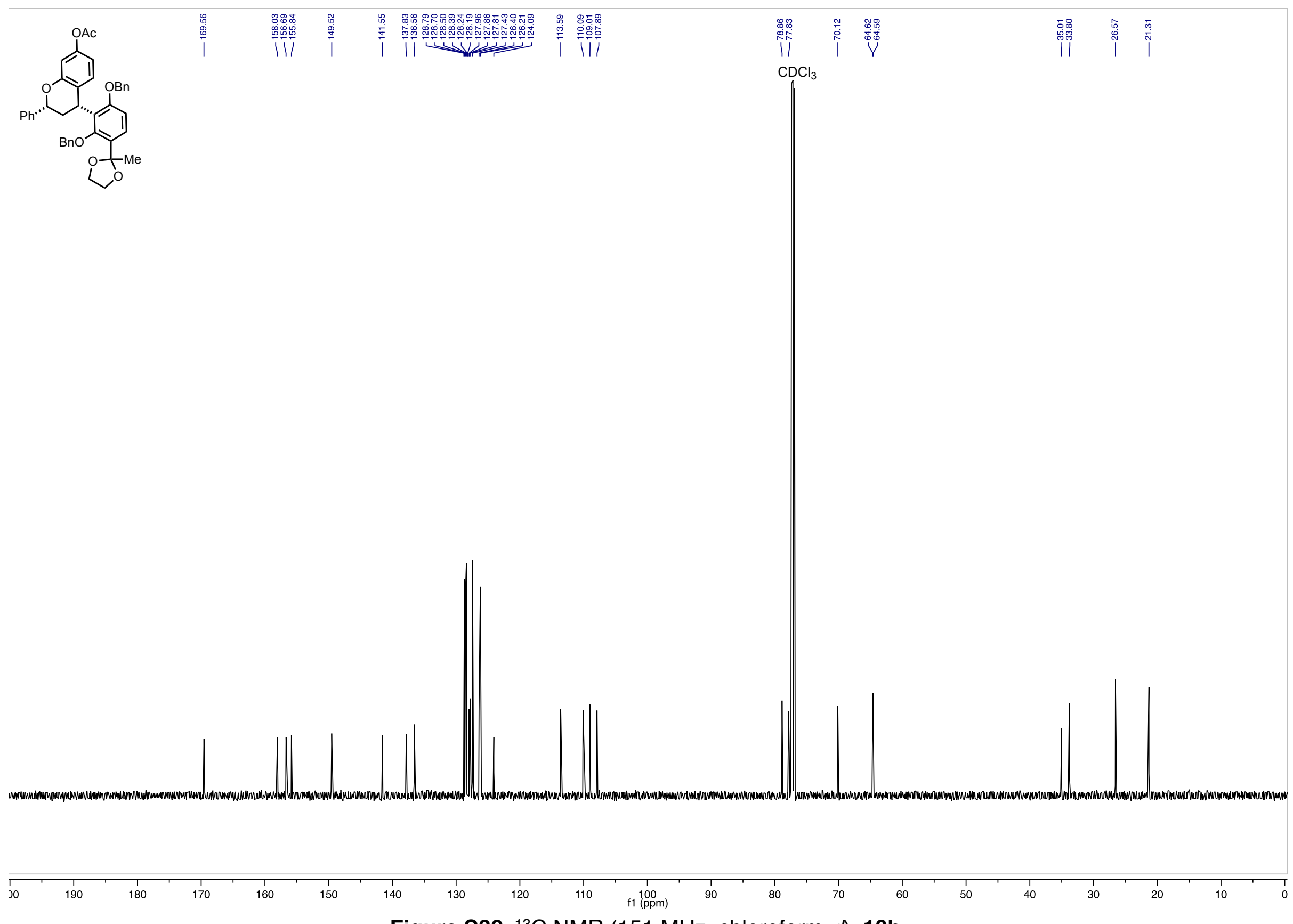

Figure S39. ${ }^{13} \mathrm{C}$ NMR (151 MHz, chloroform- $d$ ), 10b. 


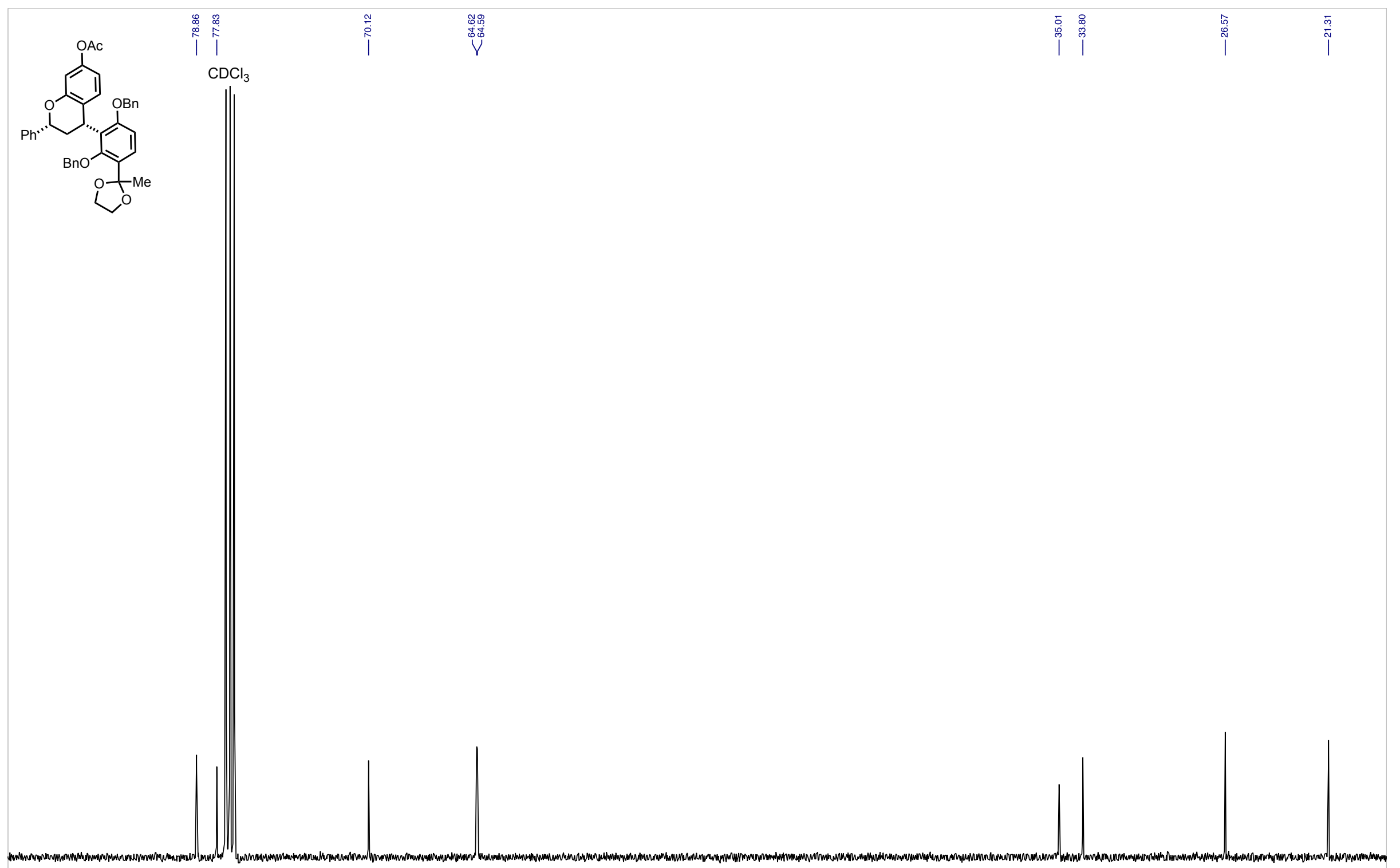

Figure S40. ${ }^{13} \mathrm{C}$ NMR (151 MHz, chloroform- $d$ ), 10b (inset). 

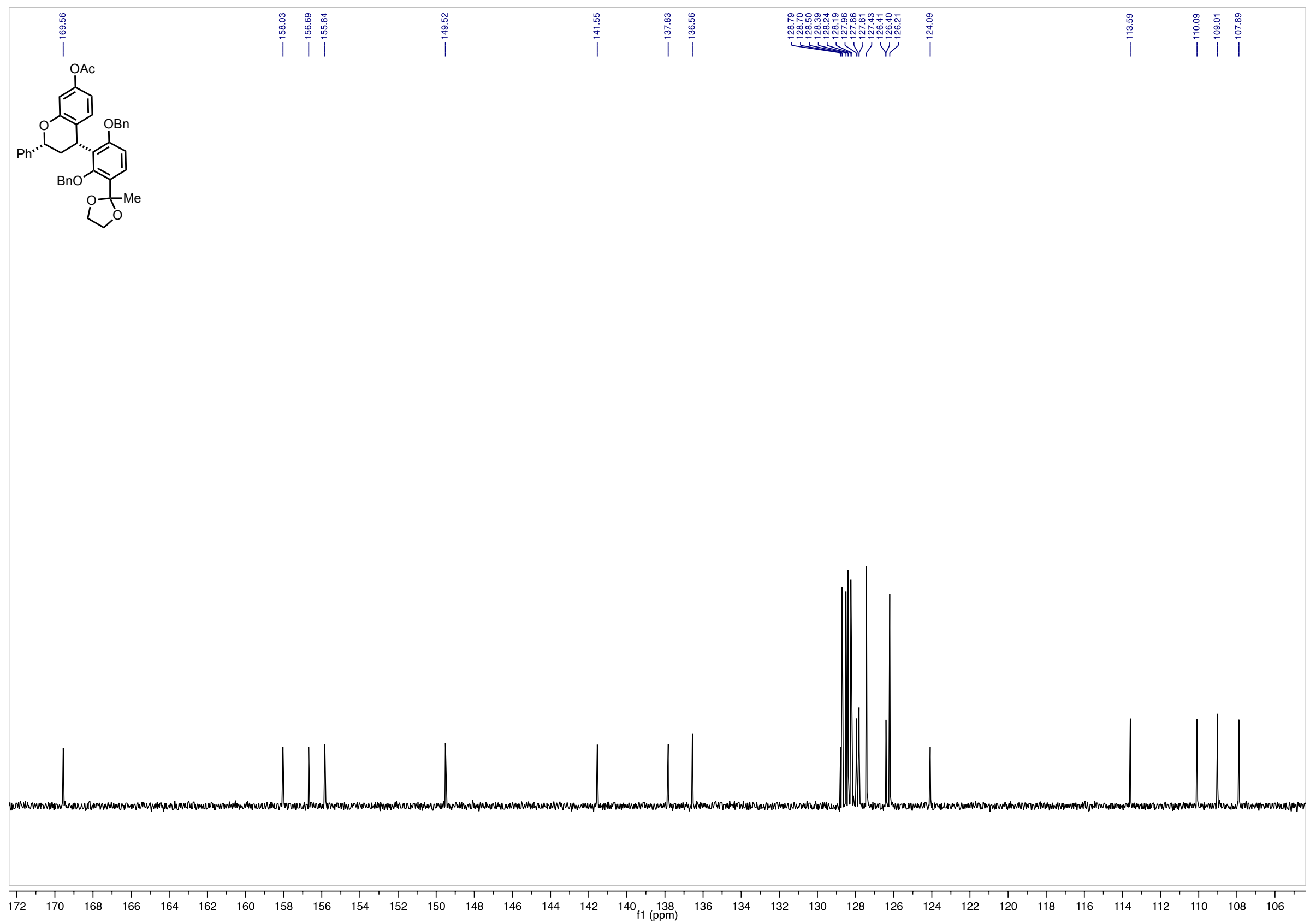

Figure S41. ${ }^{13} \mathrm{C}$ NMR (151 MHz, chloroform- $d$ ), 10b (inset). 


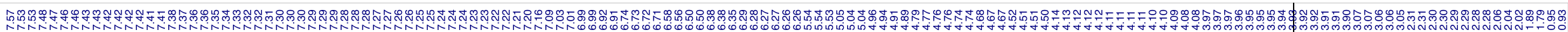
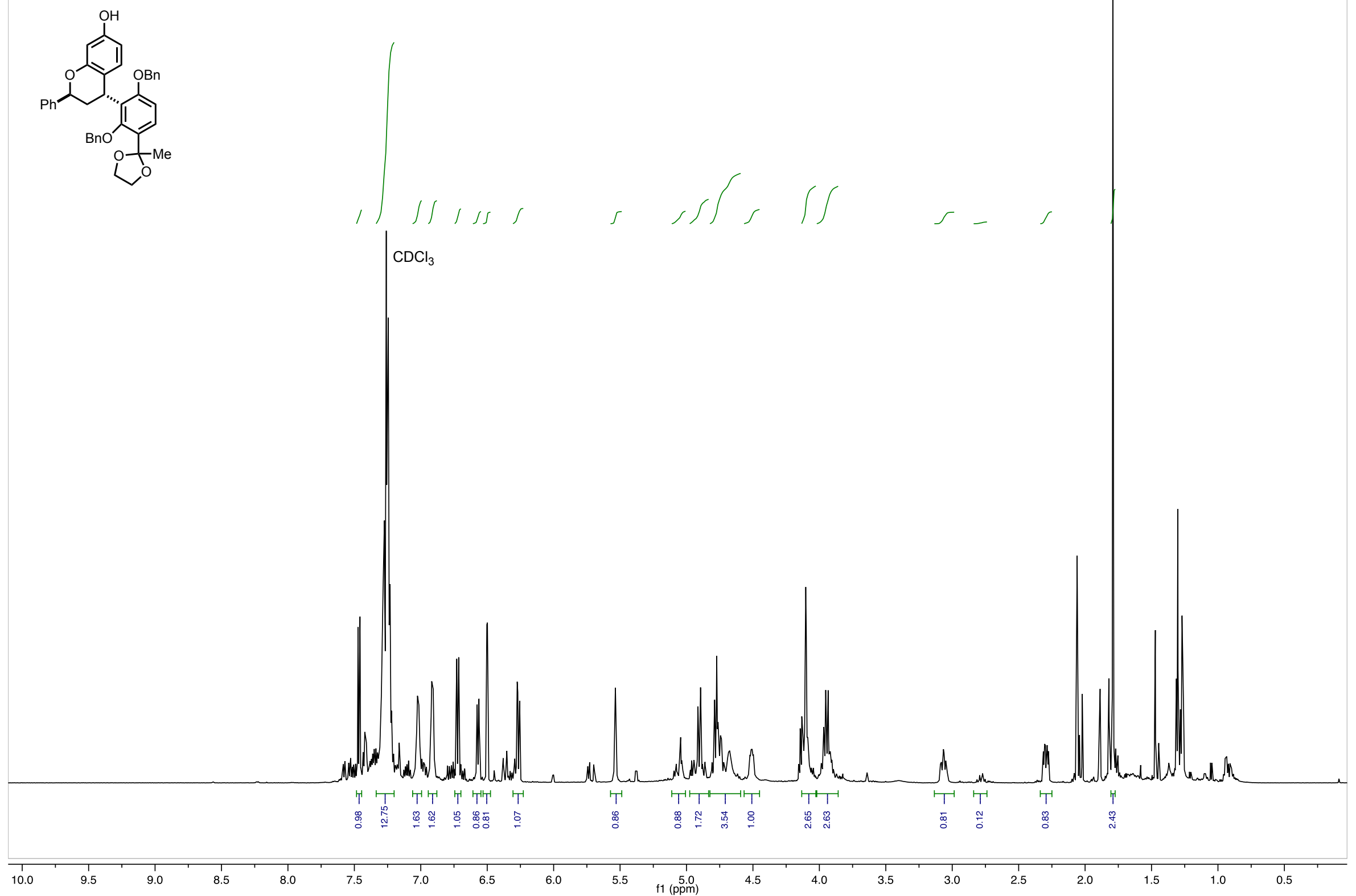

Figure S42. ${ }^{1 H}$ NMR (600 MHz, chloroform- $d$ ), 11.

S98 


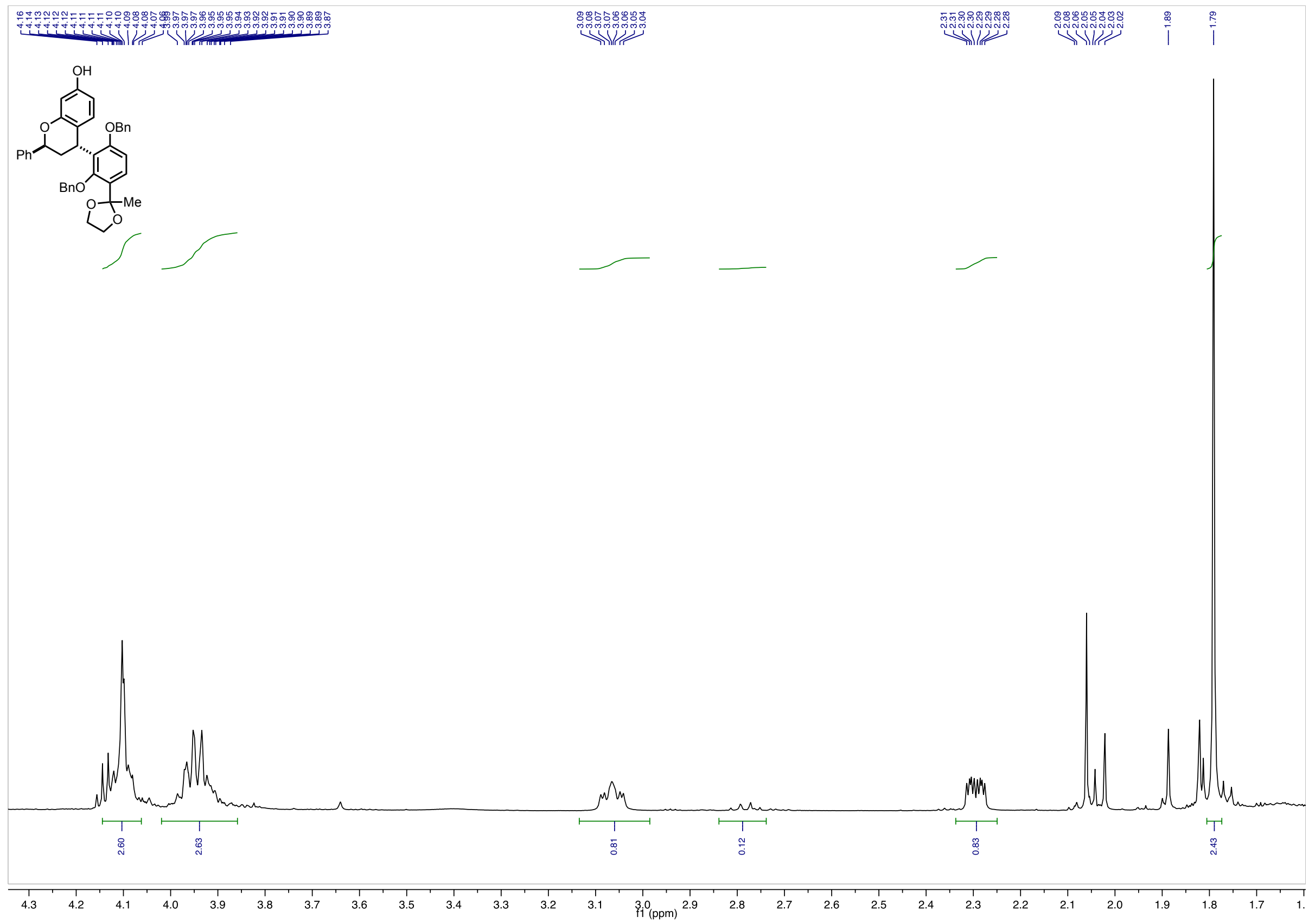

Figure S43. ${ }^{1} \mathrm{H}$ NMR (600 MHz, chloroform- $\left.d\right), 11$ (inset). 


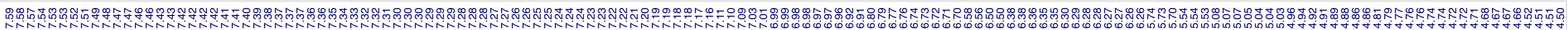<smiles>CCCOc1ccc(C2(C)OCCO2)c(OCc2ccccc2)c1[C@H]1CC(c2ccccc2)Oc2cc(O)ccc21</smiles>

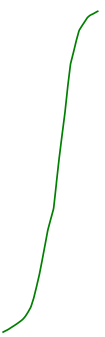

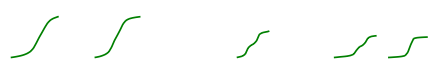

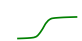
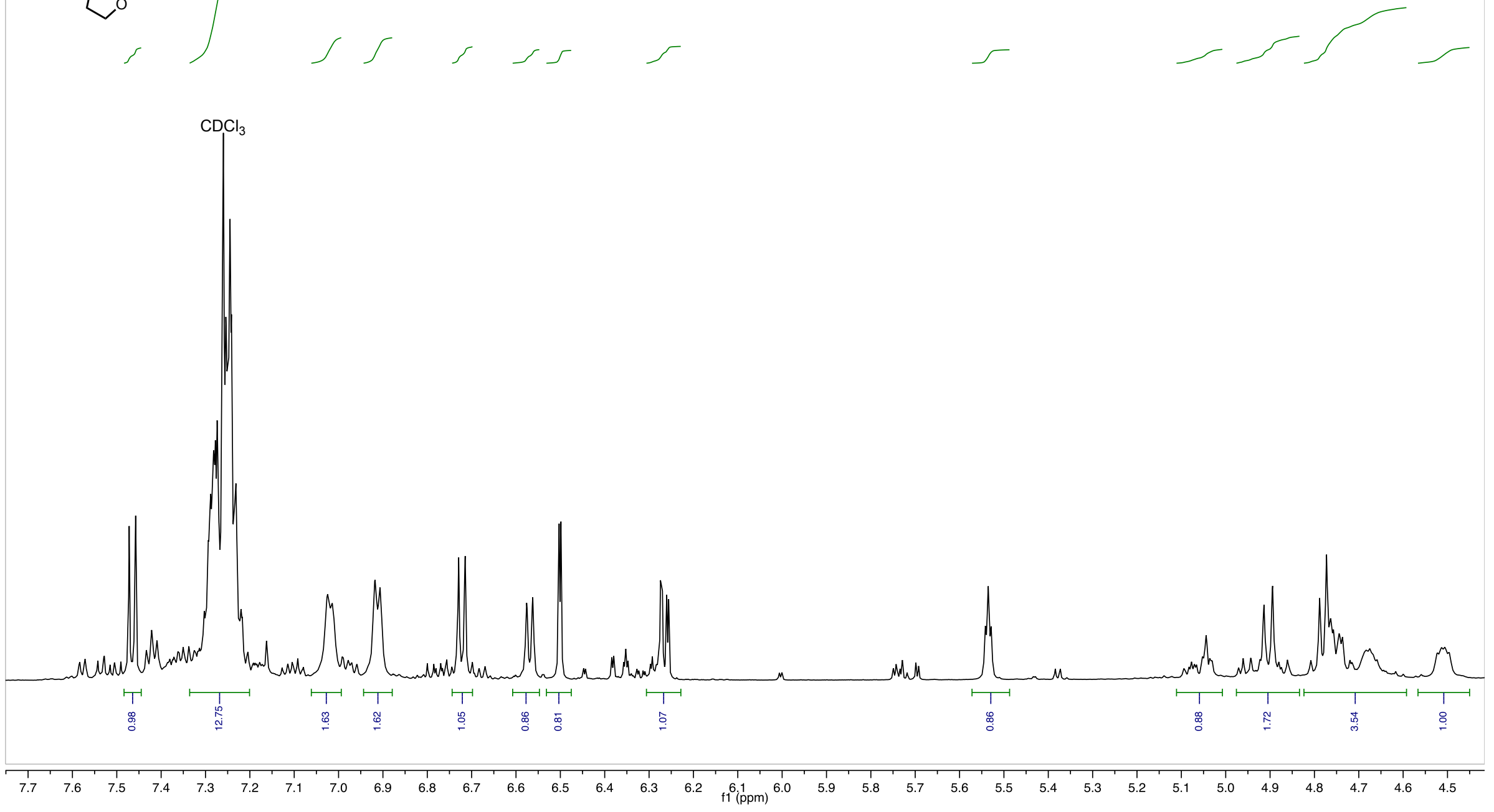

Figure S44. ${ }^{1} \mathrm{H}$ NMR (600 MHz, chloroform- $d$ ), 11 (inset).

S100 


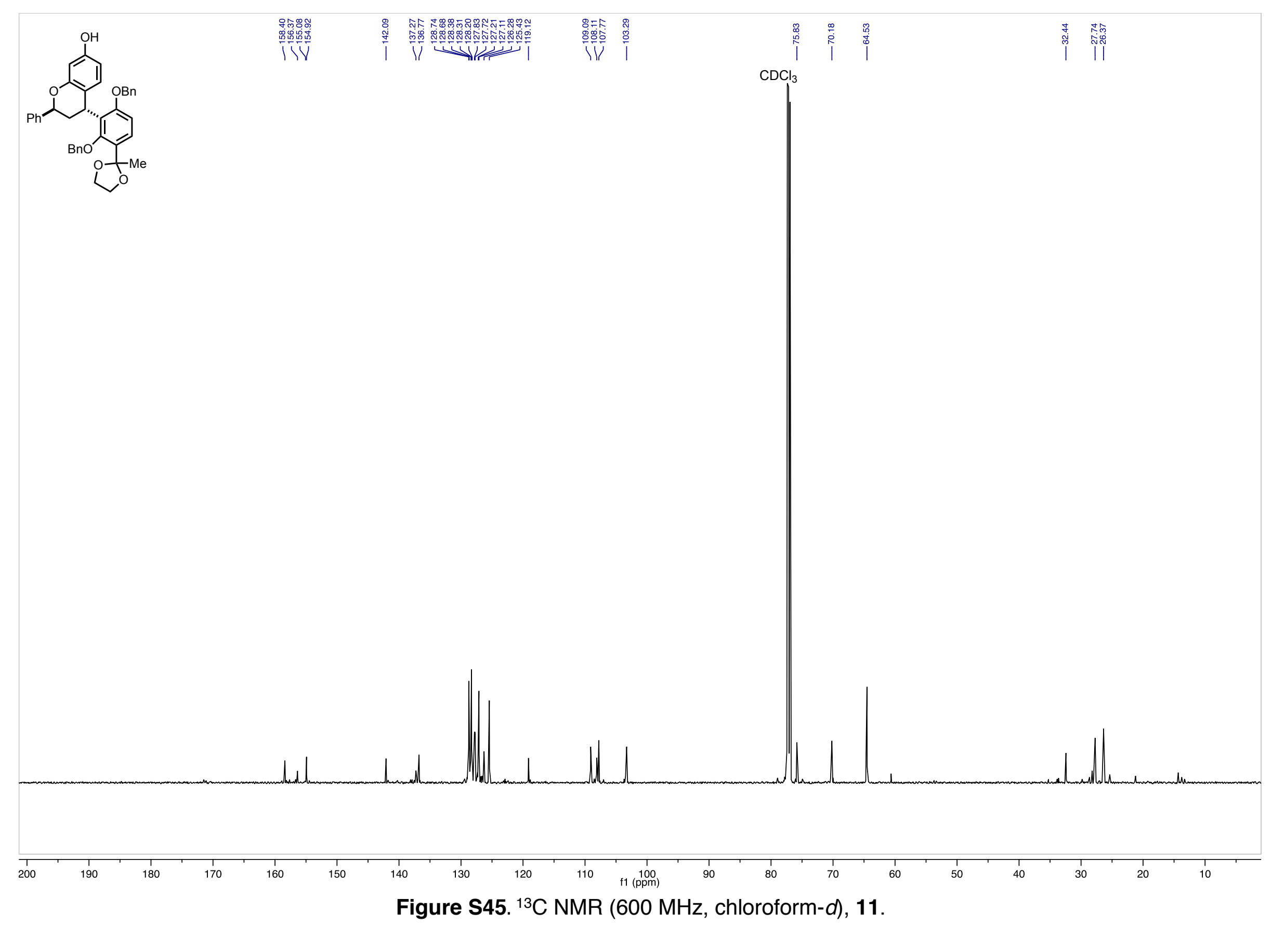

S101 

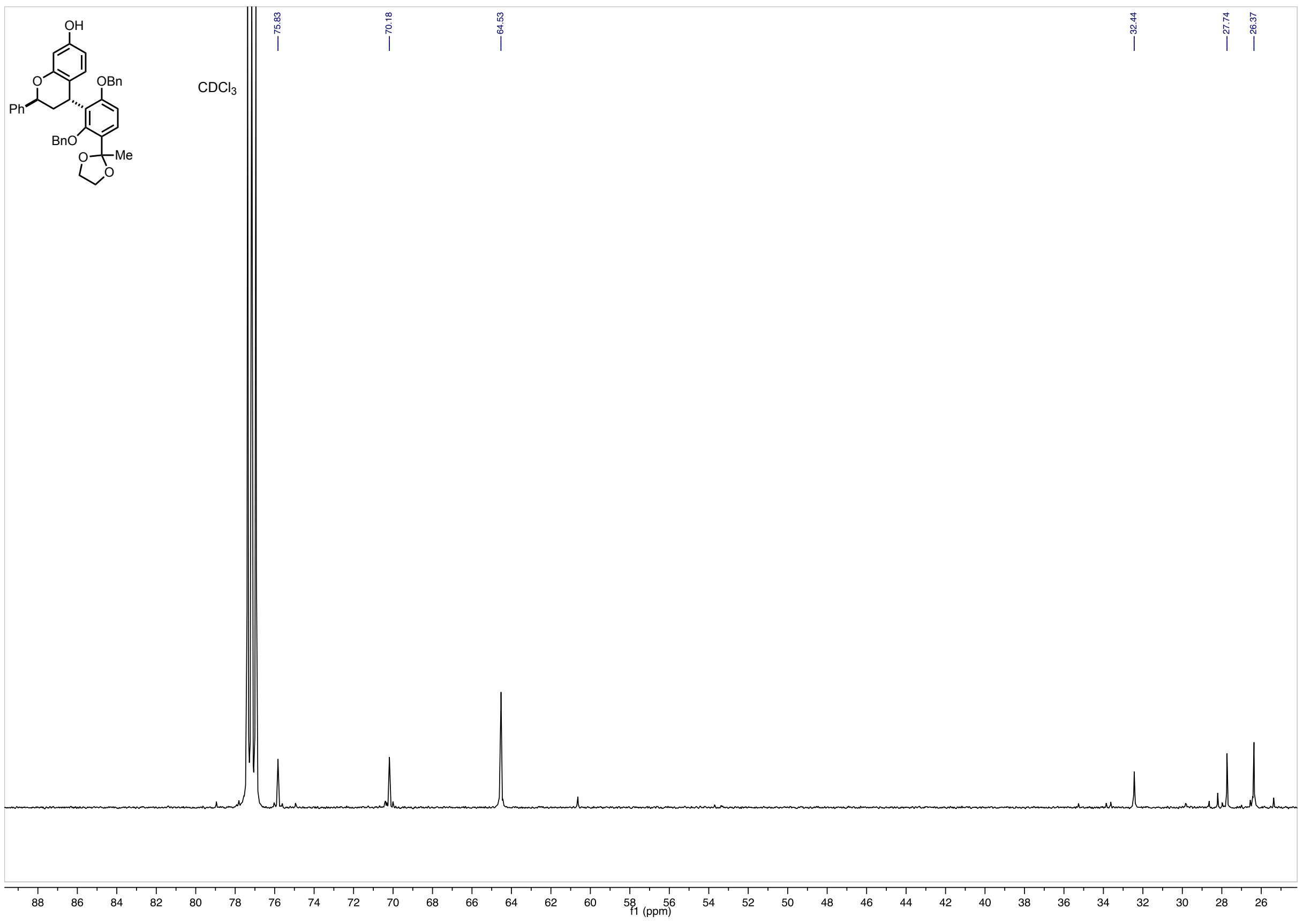

Figure S46. ${ }^{13} \mathrm{C}$ NMR (151 MHz, chloroform-d), 11 (inset). 


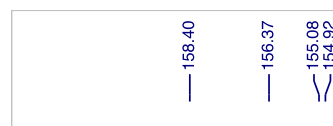

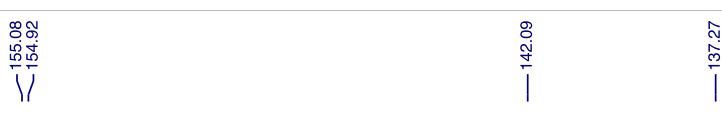
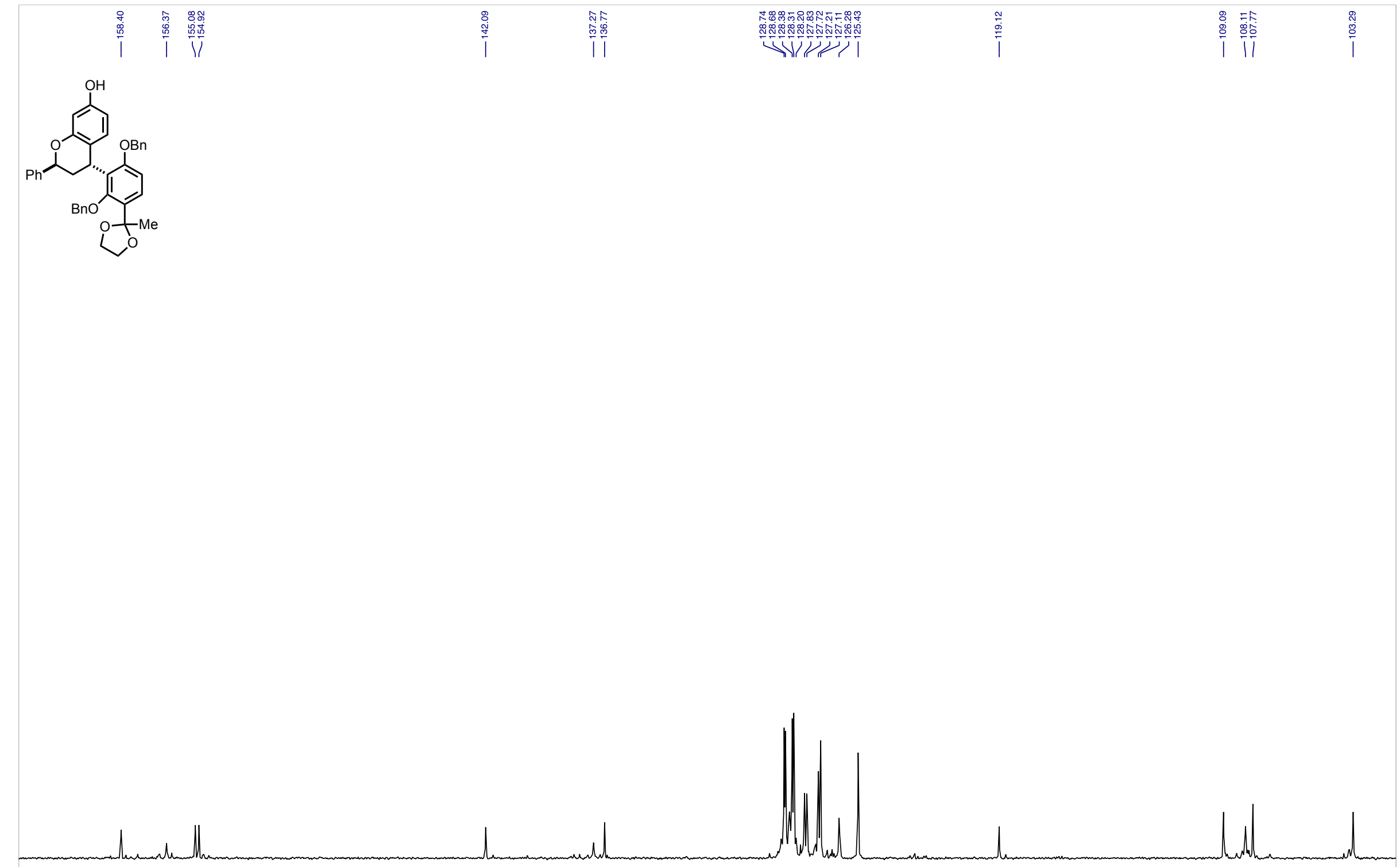

Figure S47. ${ }^{13} \mathrm{C}$ NMR (151 MHz, chloroform- $d$ ), 11 (inset). 


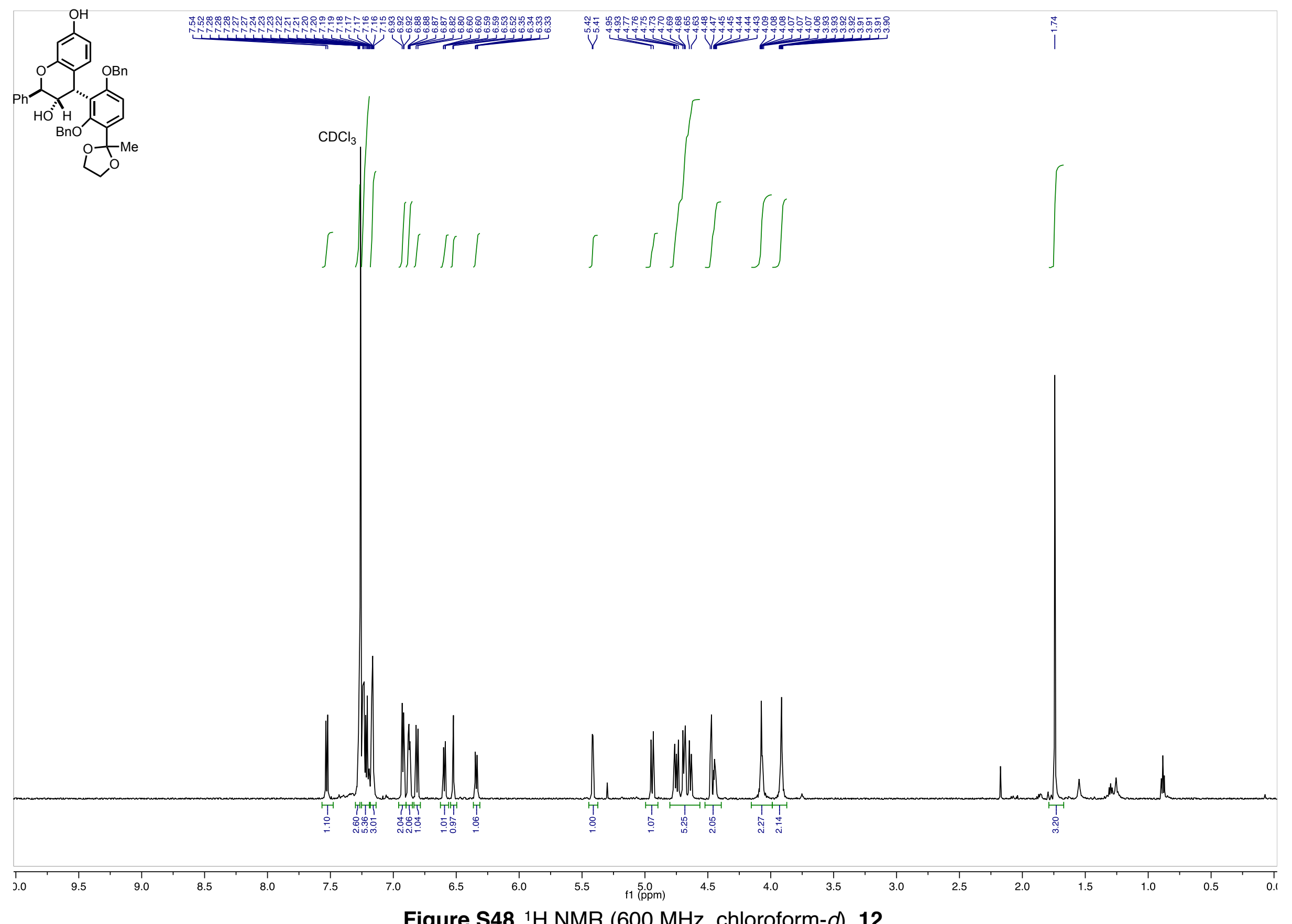

Figure S48. ${ }^{1 H} \mathrm{NMR}(600 \mathrm{MHz}$, chloroform- $d$ ), 12. 

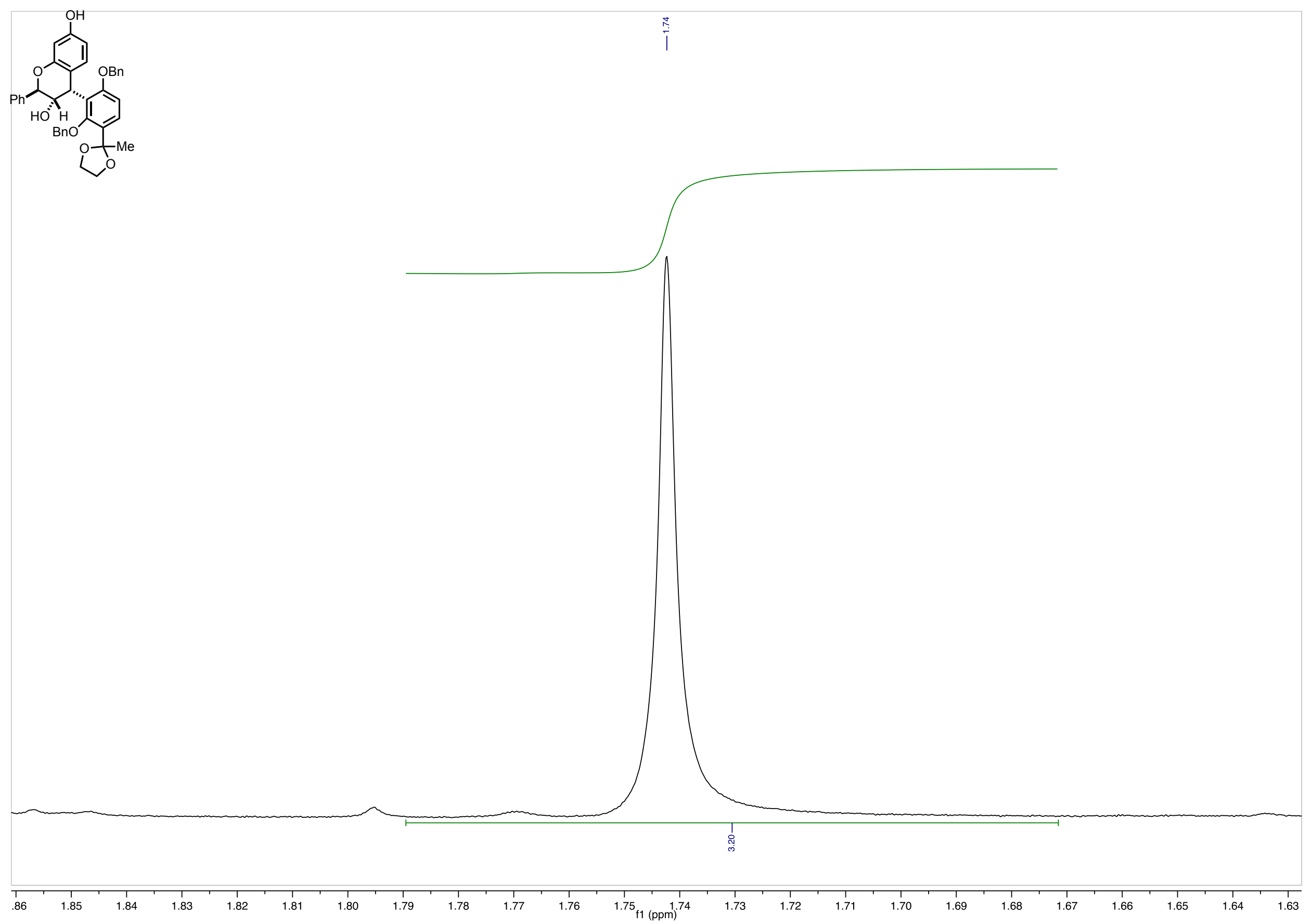

Figure S49. ${ }^{1} \mathrm{H}$ NMR (600 MHz, chloroform- $d$ ), 12 (inset). 


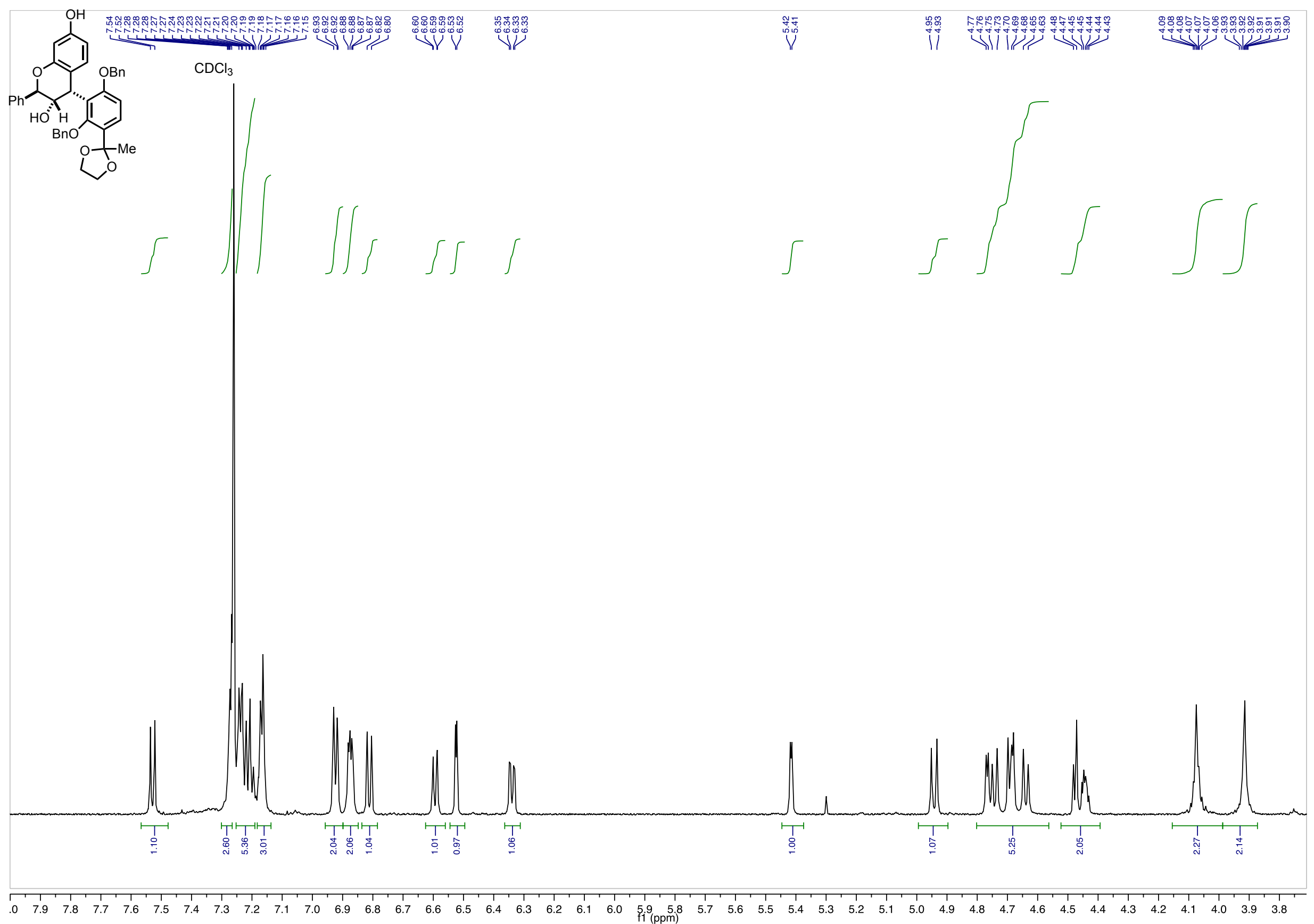

Figure S50. ${ }^{1} \mathrm{H}$ NMR (600 MHz, chloroform-d), 12 (inset). 


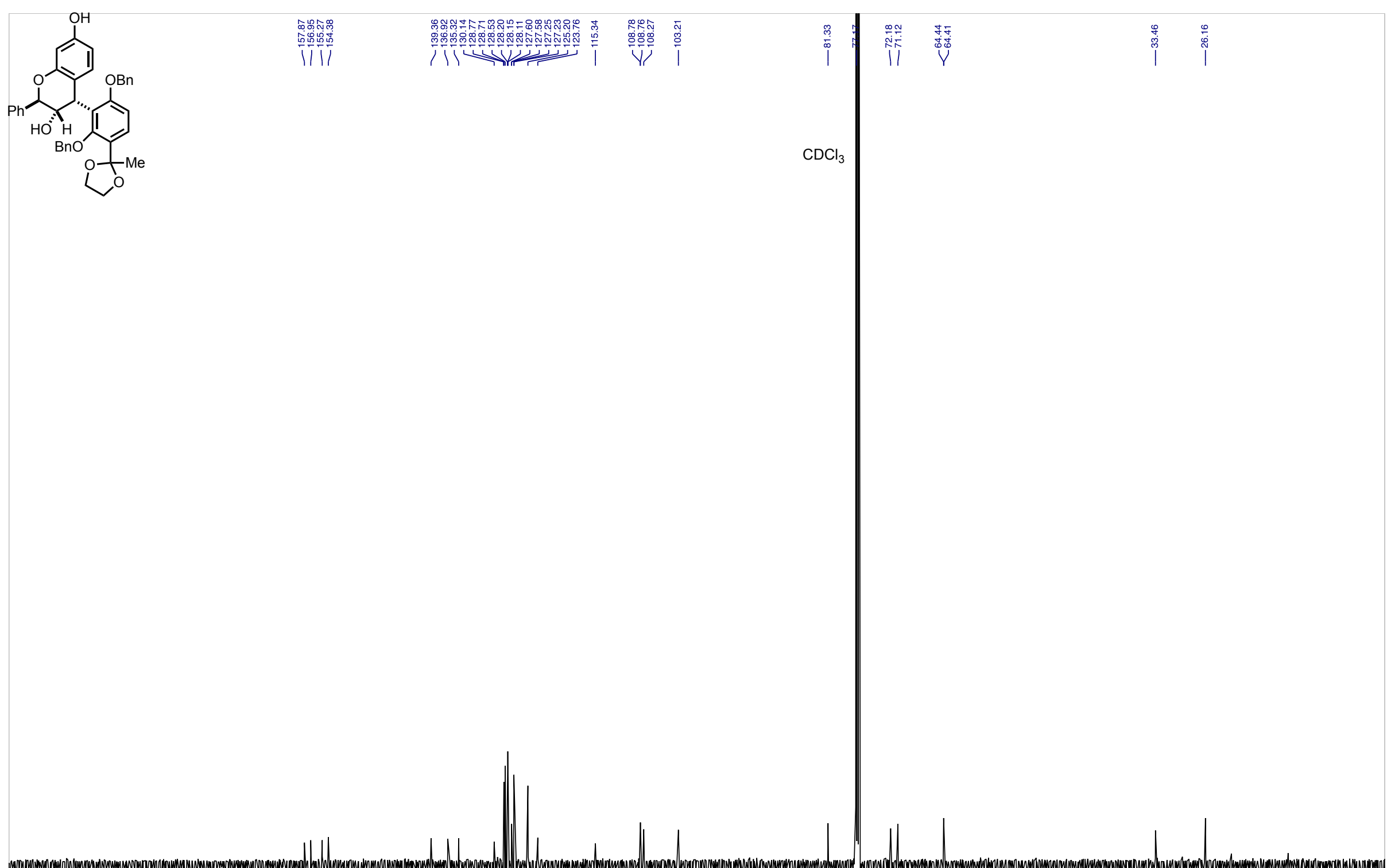

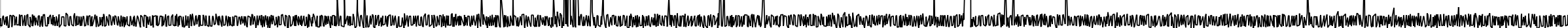

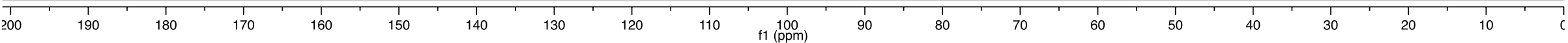

Figure S51. ${ }^{13} \mathrm{C}$ NMR (151 MHz, chloroform- $d$ ), 12. 


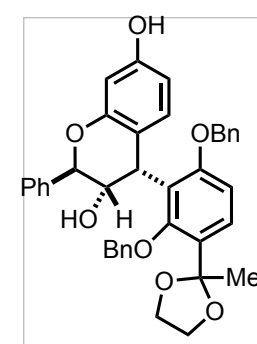

$$
\text { ì }
$$

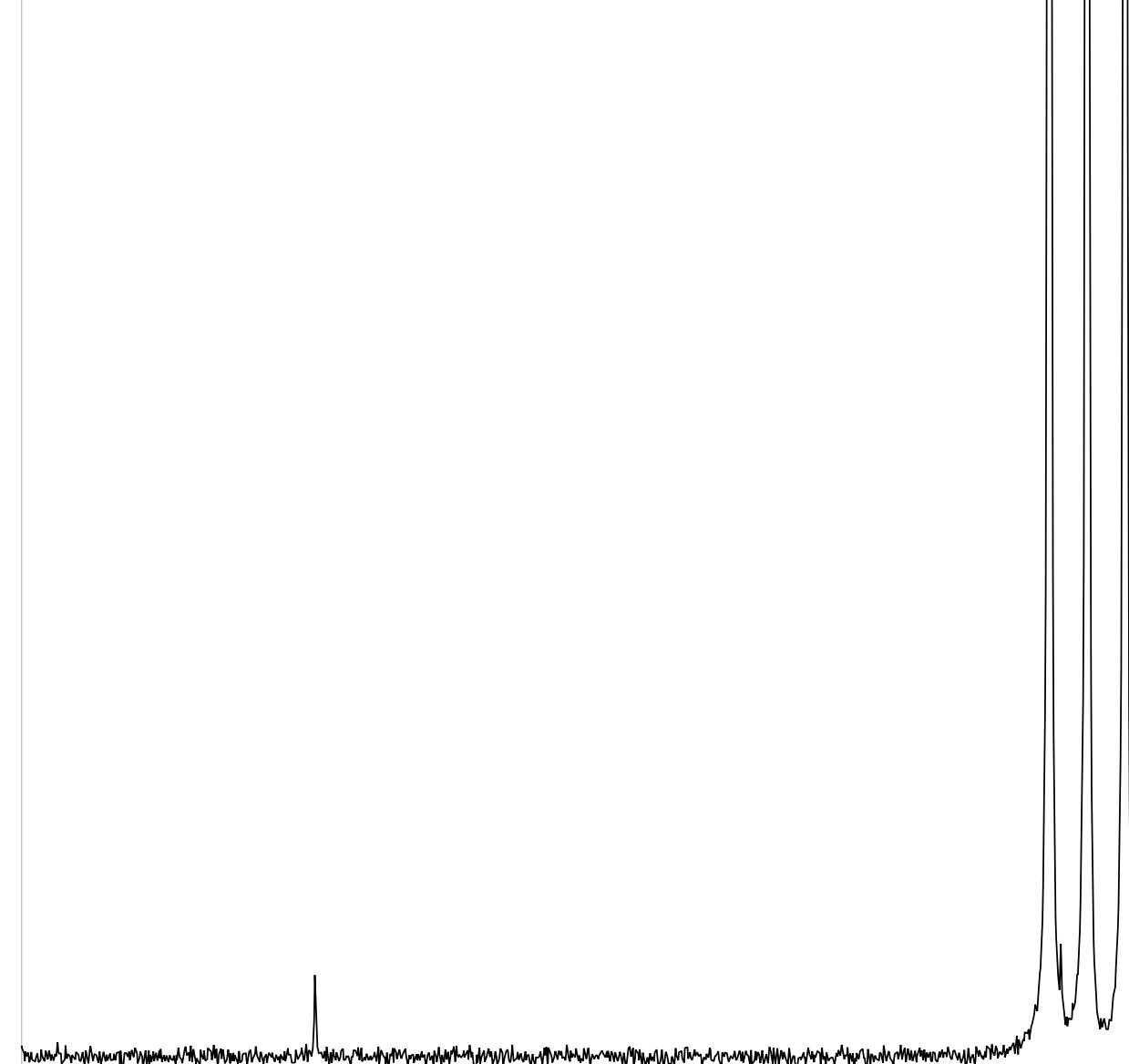

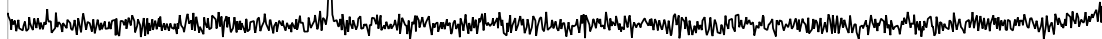



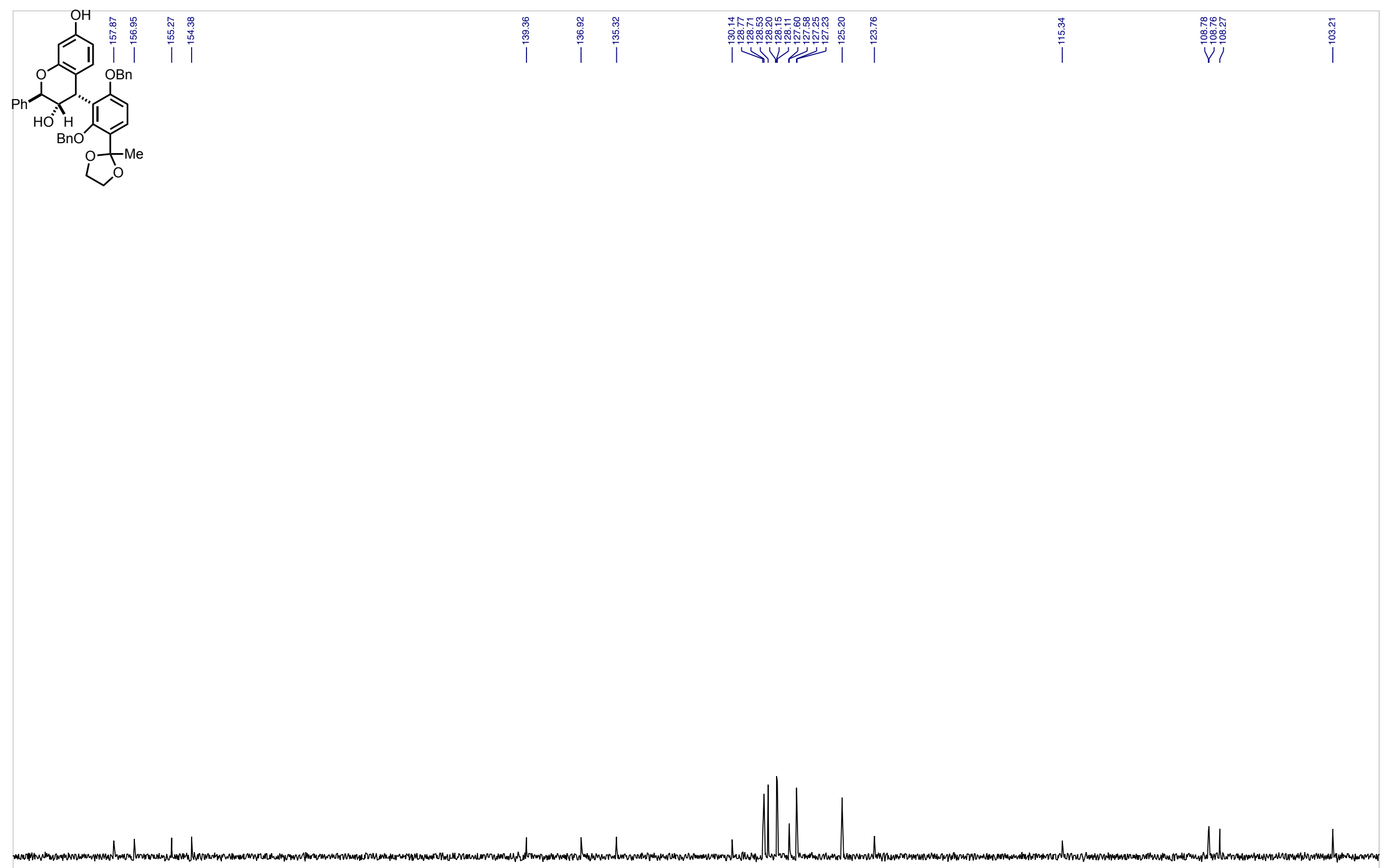

Figure S53. ${ }^{13} \mathrm{C}$ NMR (151 MHz, chloroform-d), 12 (inset). 

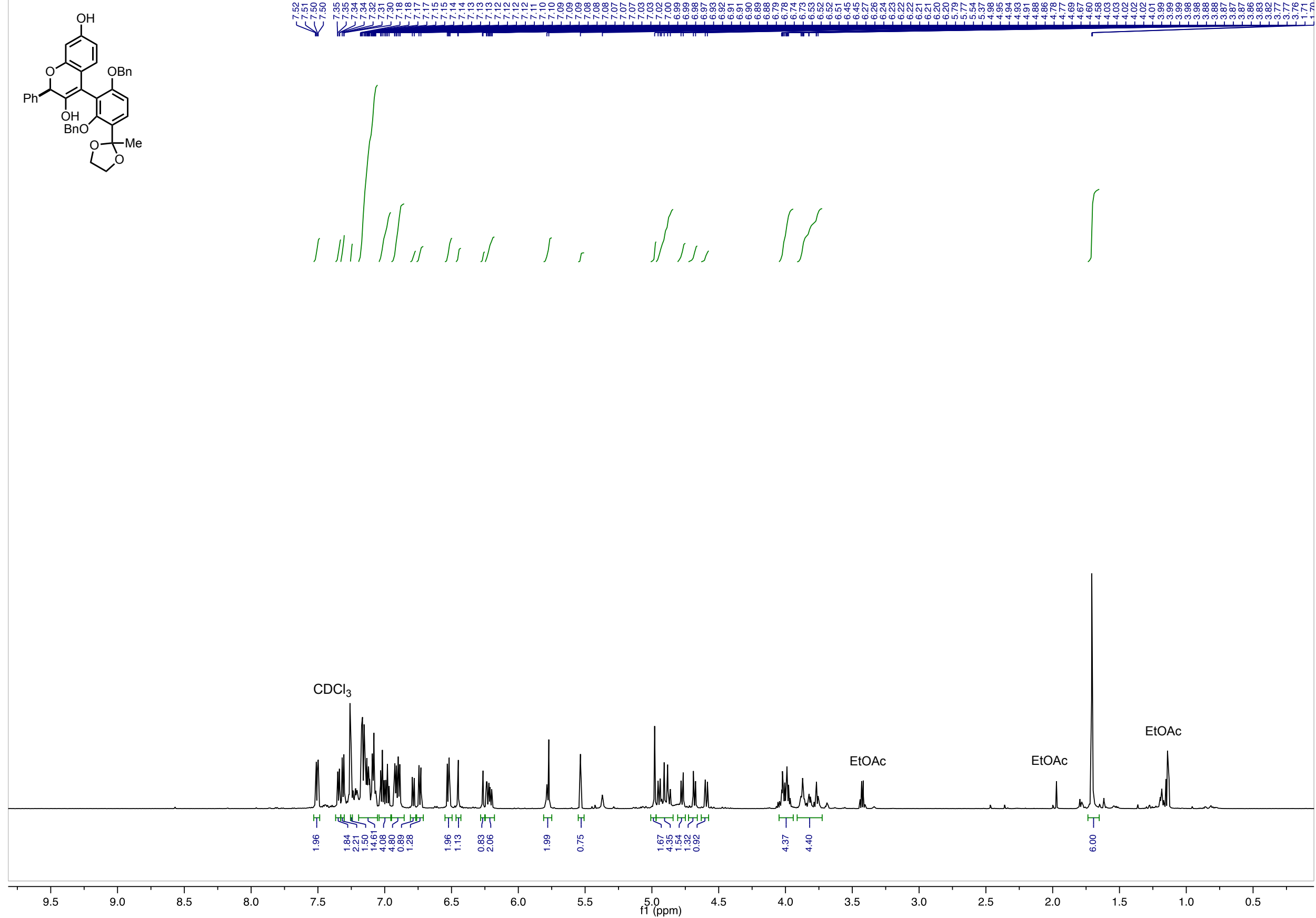

Figure S54. ${ }^{1} \mathrm{H}$ NMR $(600 \mathrm{MHz}$, chloroform- $d), 13$ (crude spectrum). 

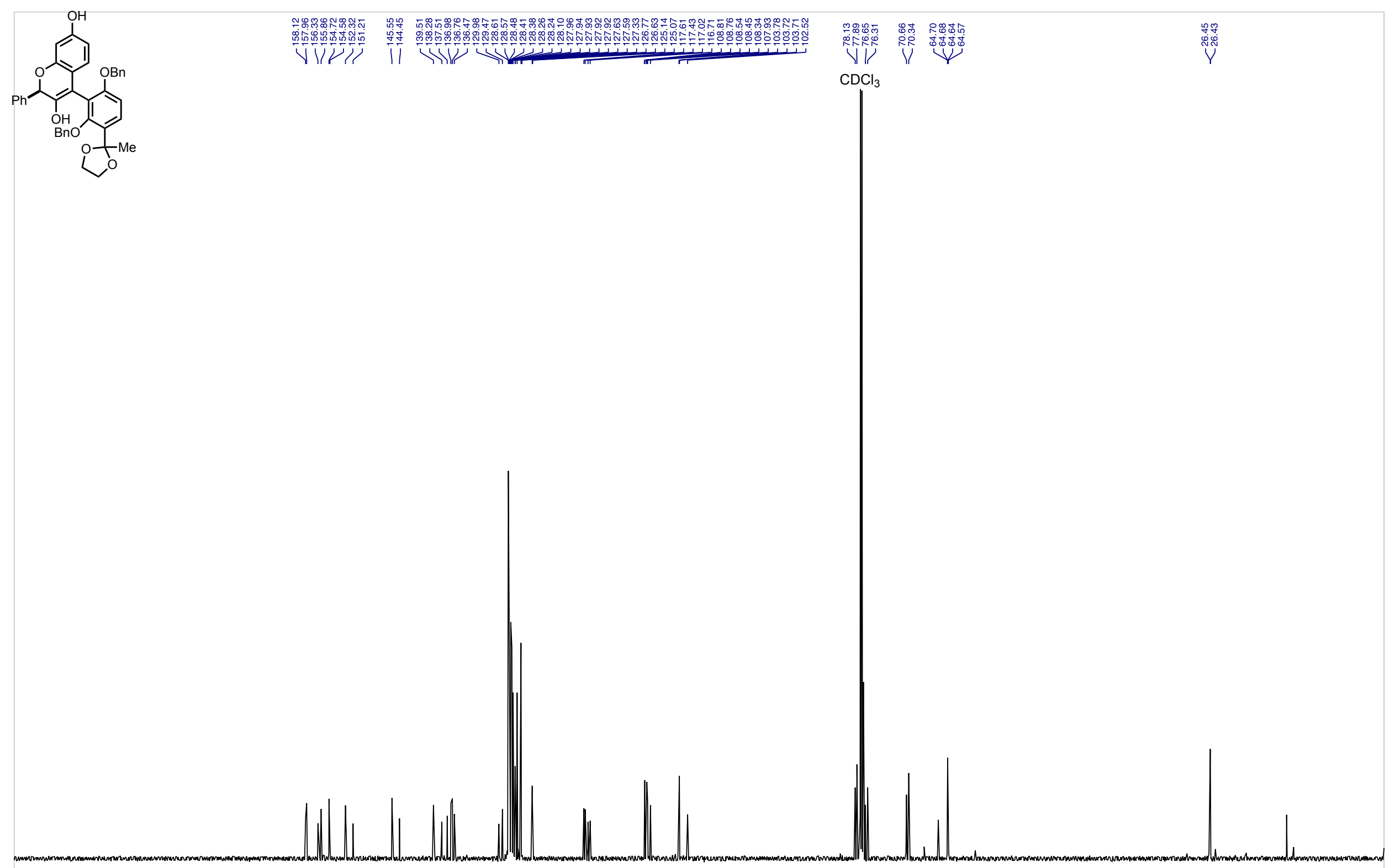

Figure S55. ${ }^{13} \mathrm{C}$ NMR (151 MHz, chloroform- $d$ ), 13 (crude spectrum). 

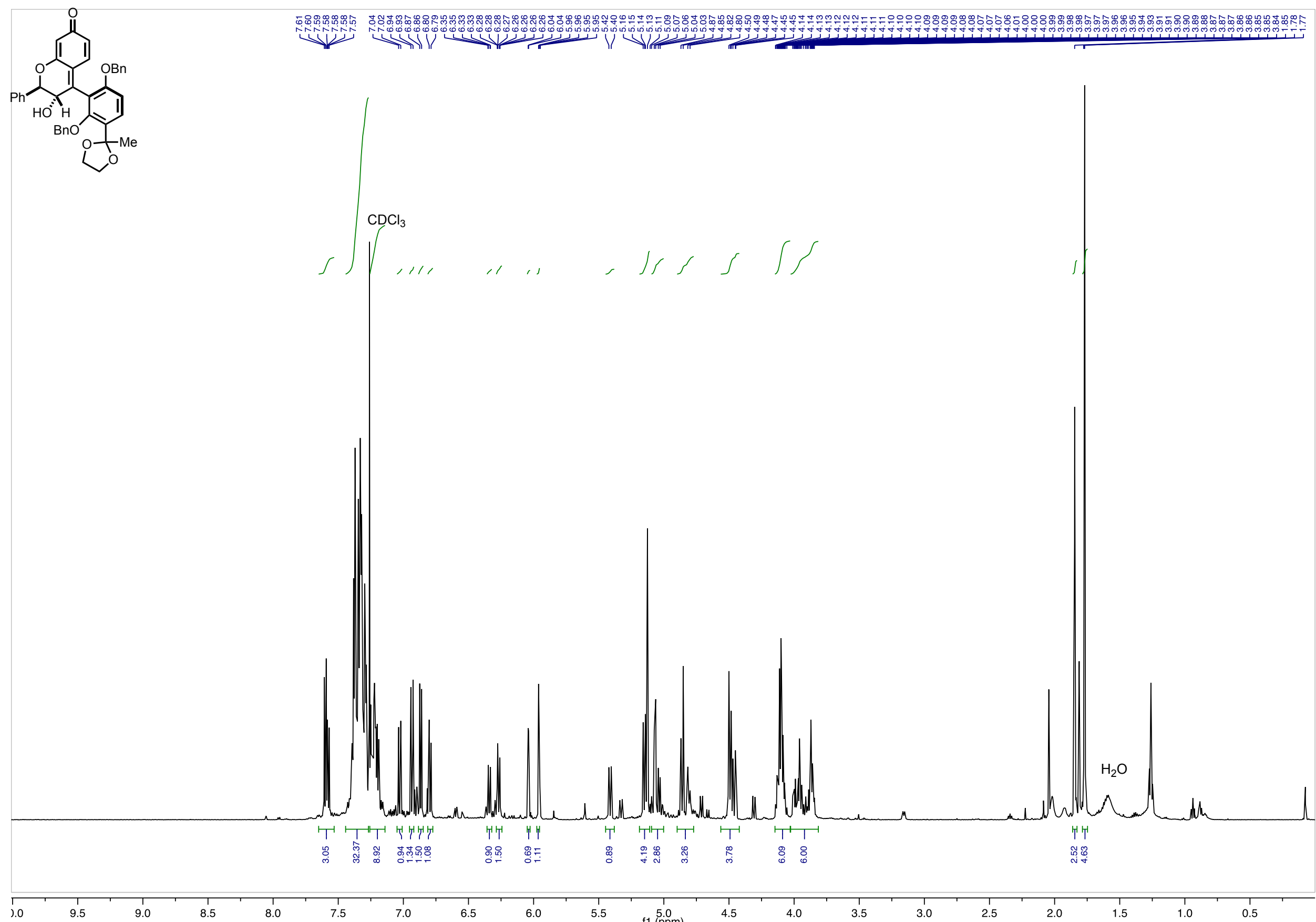

Figure S56. ${ }^{1} \mathrm{H}$ NMR (600 MHz, chloroform-d), 14.

S112 


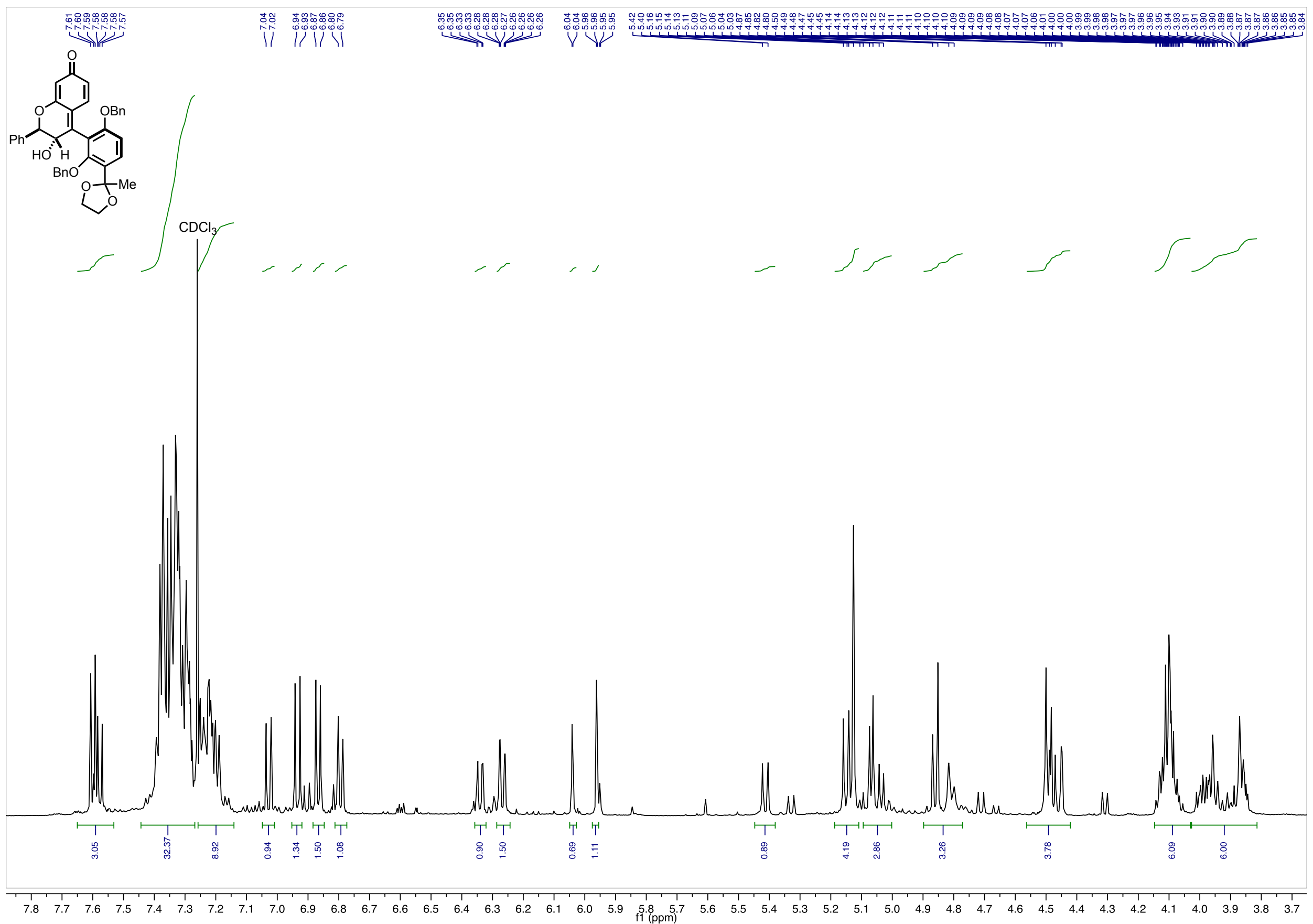

Figure S57. ${ }^{1} \mathrm{H}$ NMR (600 MHz, chloroform- $d$ ), 14 (inset).

$\mathrm{S} 113$ 


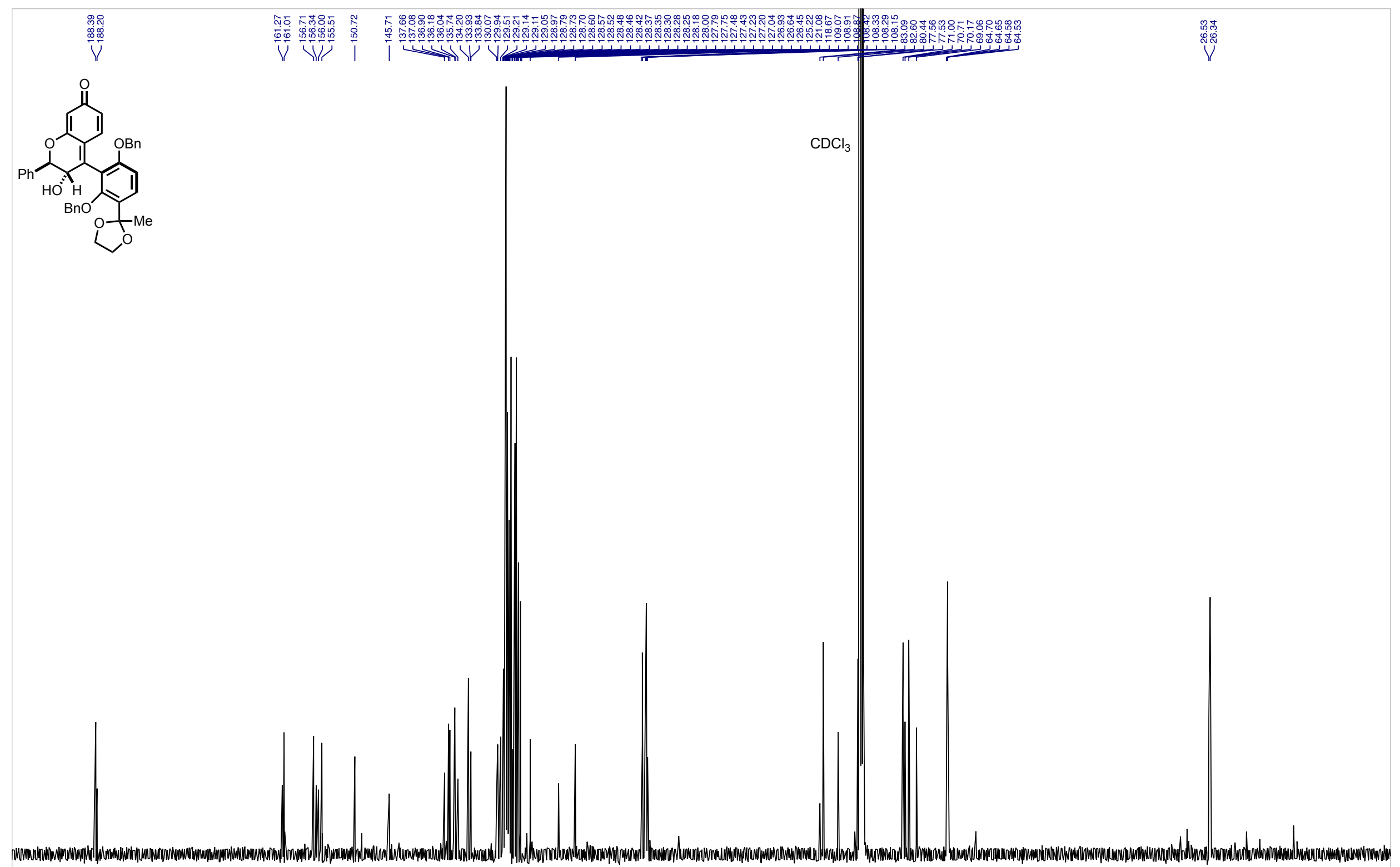

190 180 170 160 150 140 130 120 110 f1 $\left(\begin{array}{c}100 \\ \mathrm{ppm})\end{array}\right.$ $\begin{array}{lll}1 & 1 \\ 90 & 80 & 70\end{array}$ $\begin{array}{lll}70 & 1 & \\ 60 & 50\end{array}$ 40 30 20 10

Figure S58. ${ }^{13} \mathrm{C}$ NMR (151 MHz, chloroform-d), 14. 


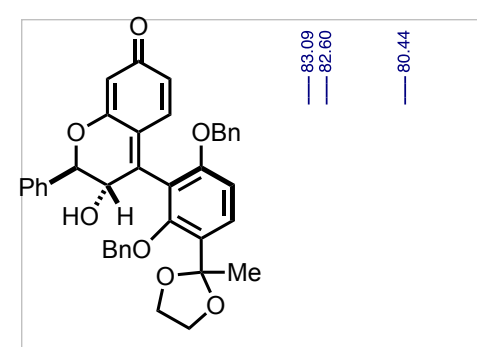

$\mathrm{CDCl}_{3}$
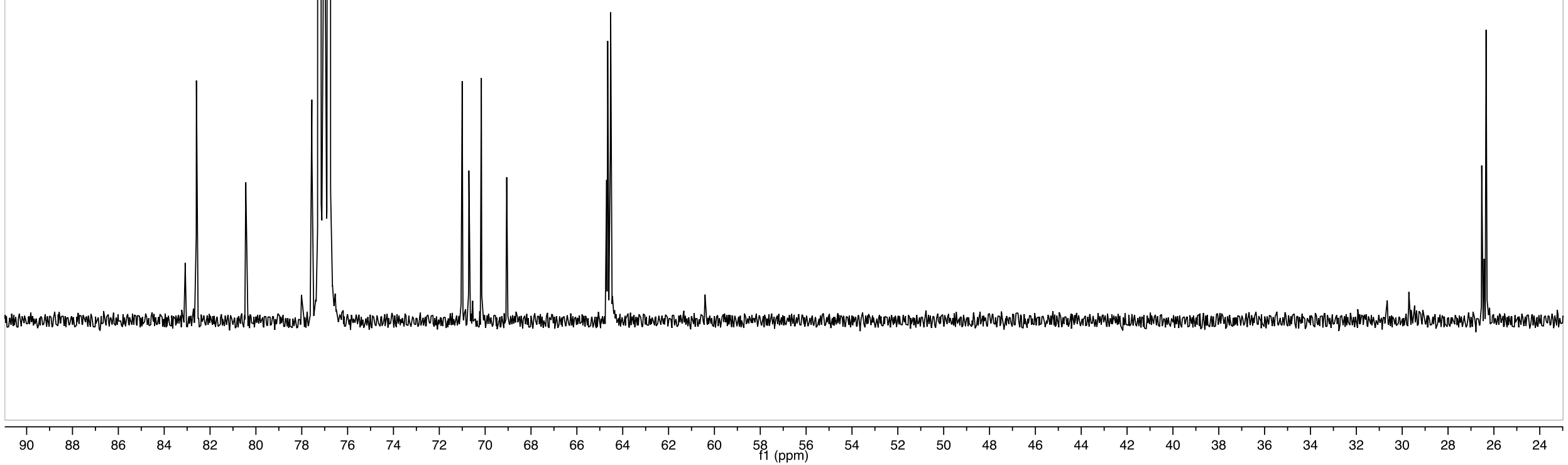

Figure S59. ${ }^{13} \mathrm{C}$ NMR (151 MHz, chloroform-d), 14 (inset). 


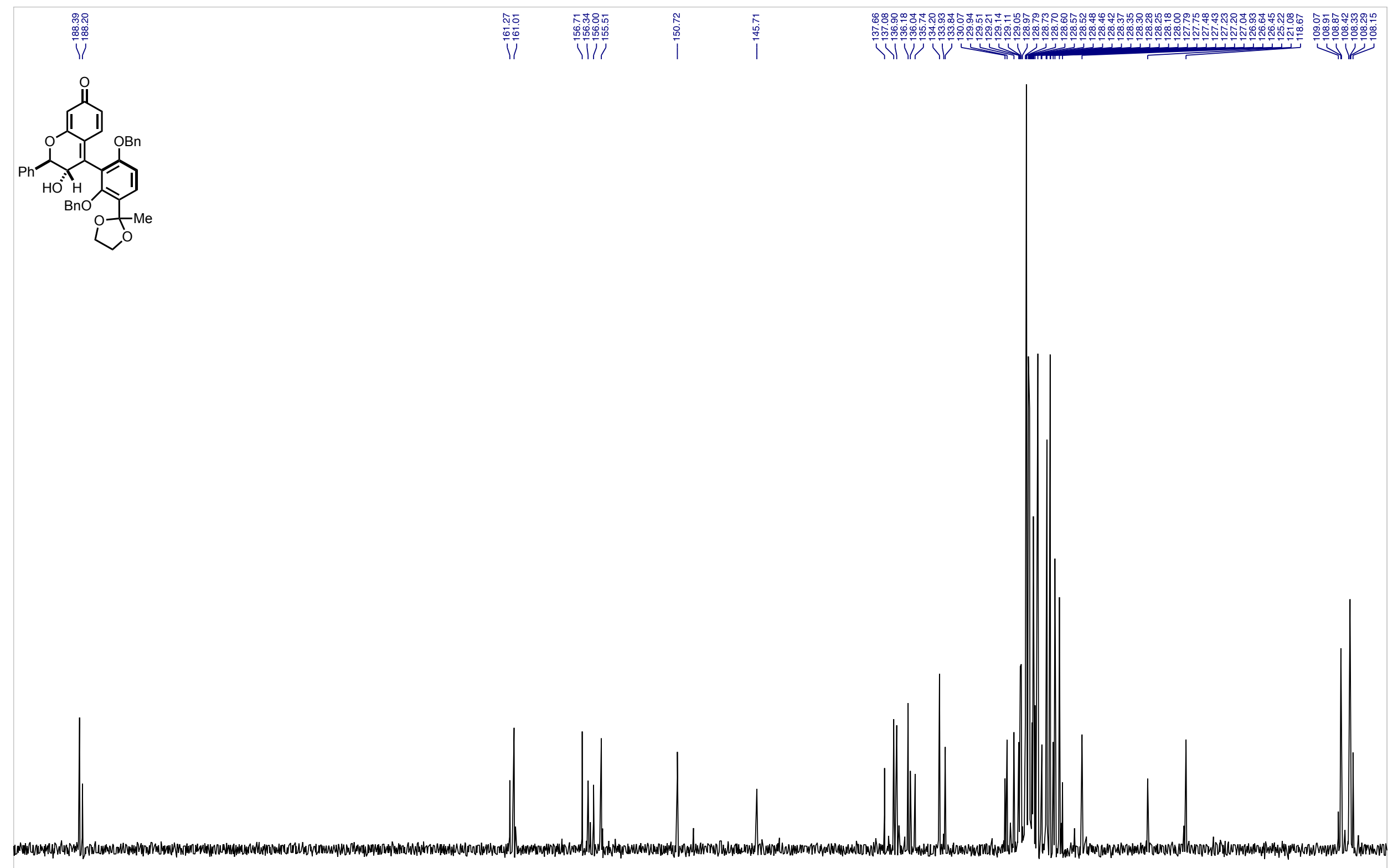

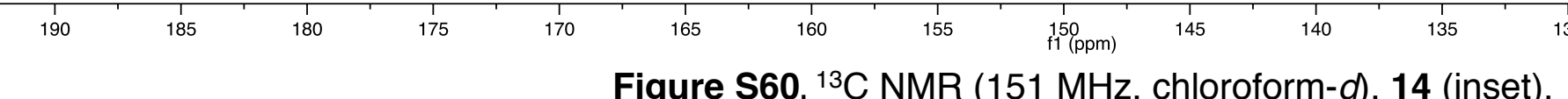

S116 


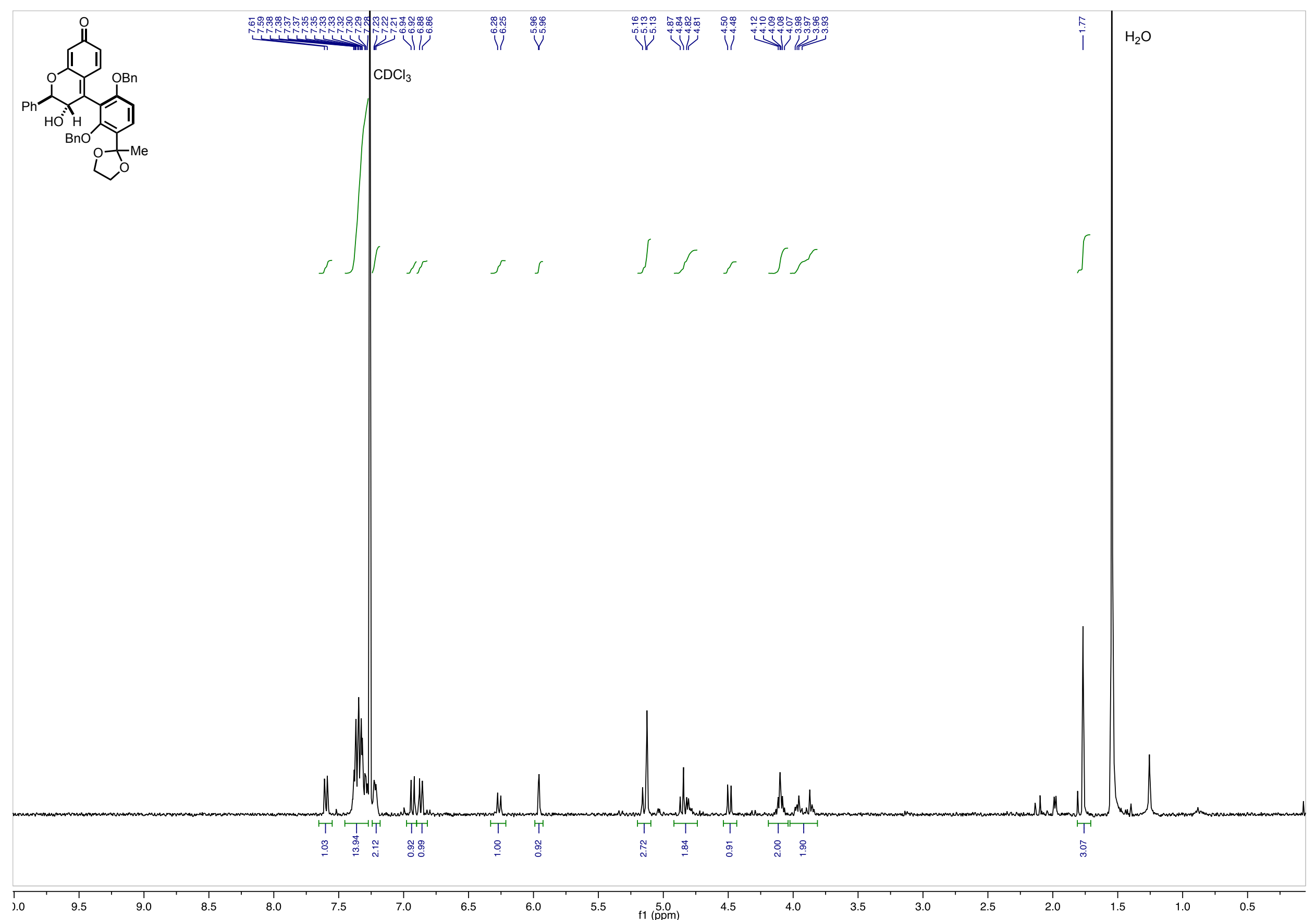

Figure S61. ${ }^{1} \mathrm{H} \mathrm{NMR}(600 \mathrm{MHz}$, chloroform- $d)$, 14, less polar atropisomer. 


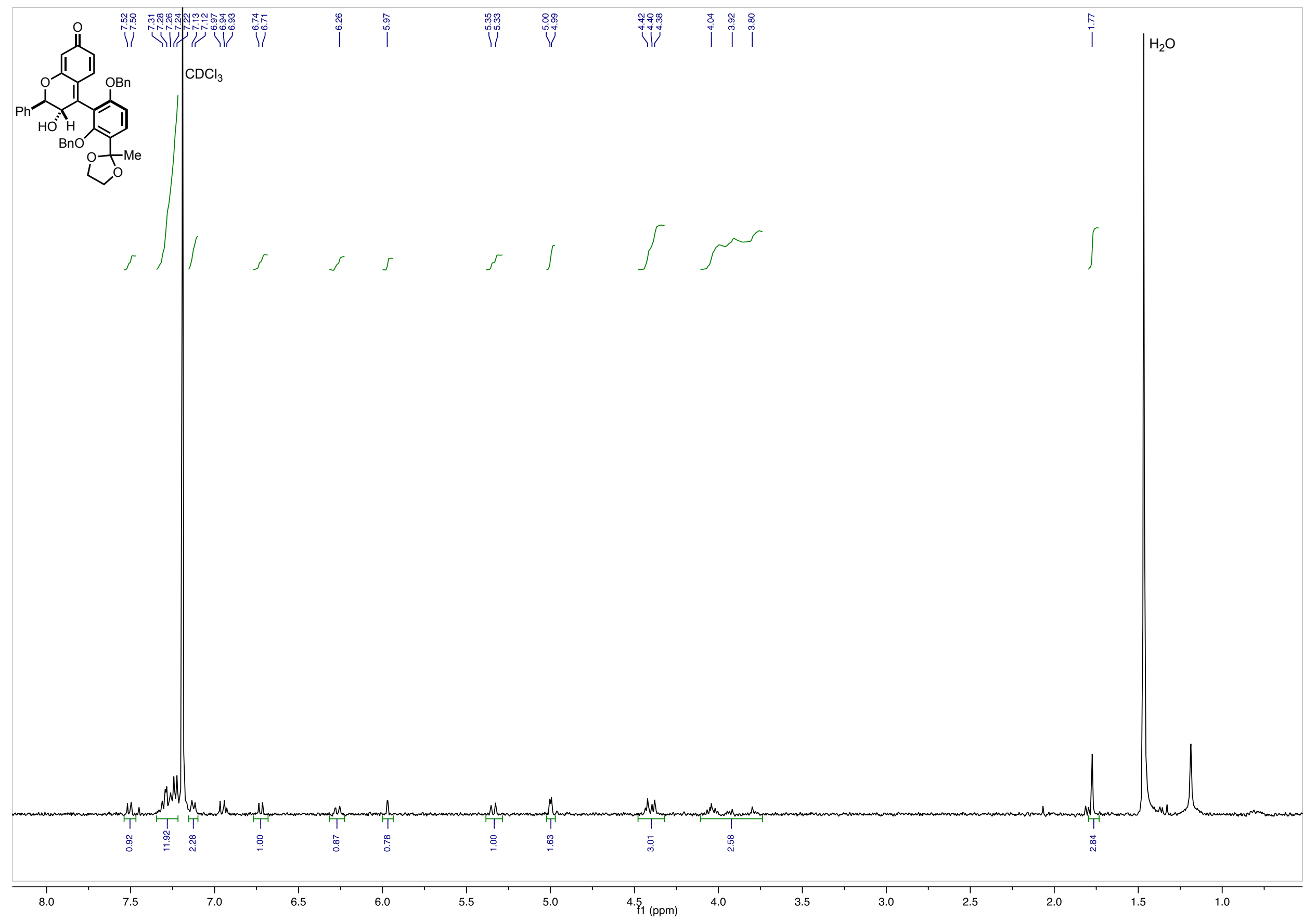

Figure S62. ${ }^{1} \mathrm{H}$ NMR $(600 \mathrm{MHz}$, chloroform- $d)$, 14, more polar atropisomer.

S118 


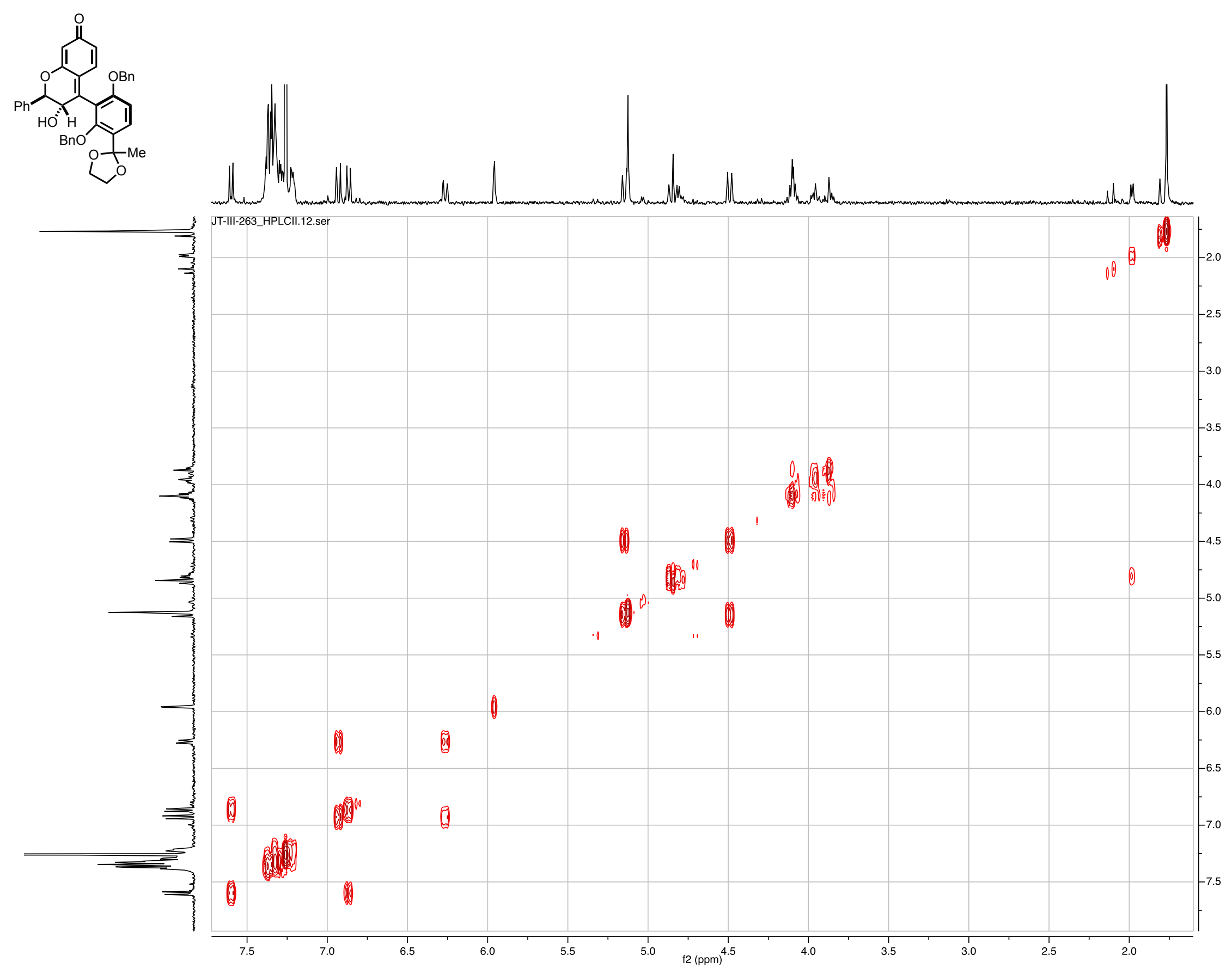

Figure S63. COSY NMR spectrum, 14, less polar diastereomer. 


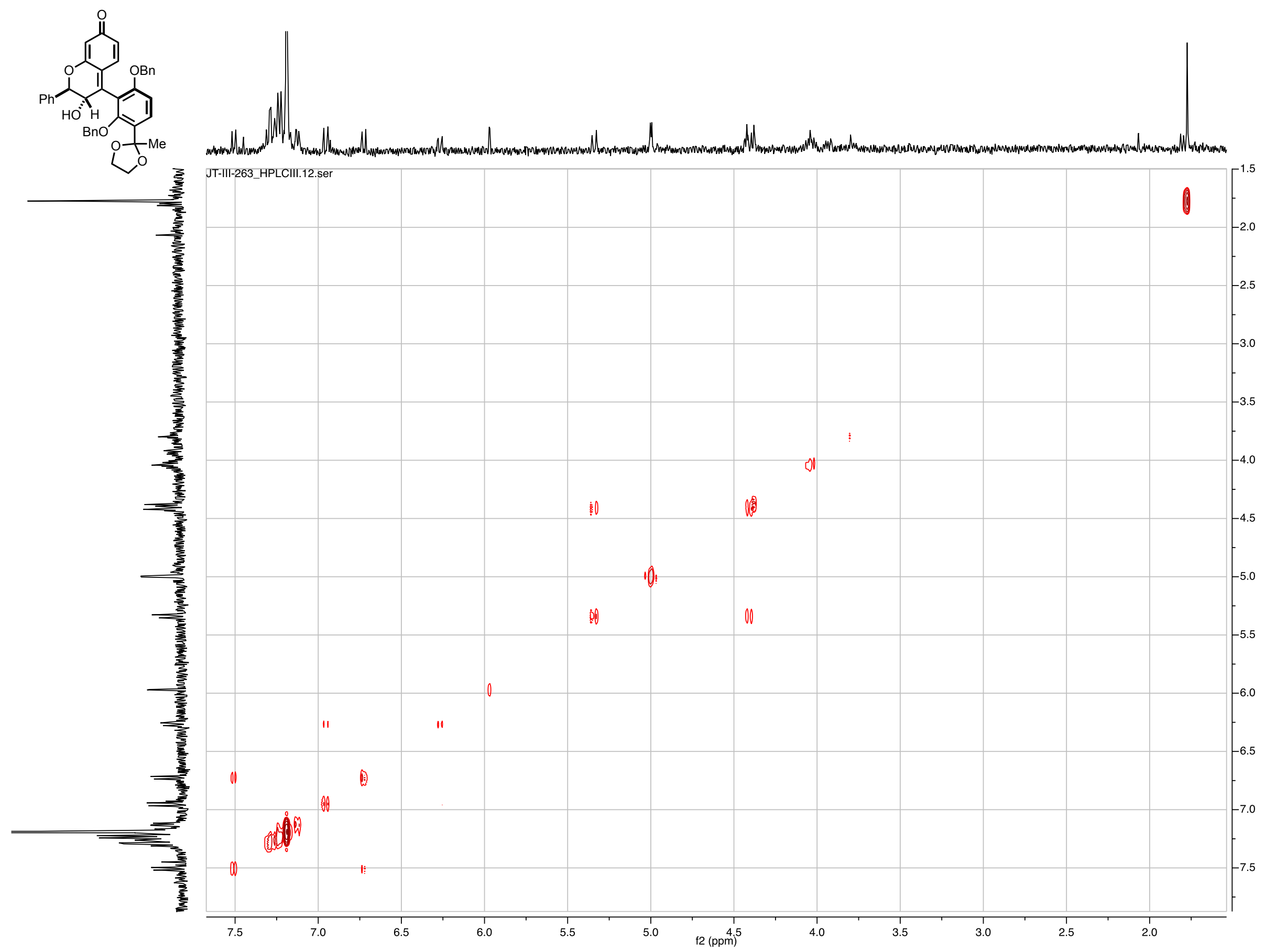

Figure S64. COSY NMR spectrum, 14, more polar diastereomer. 

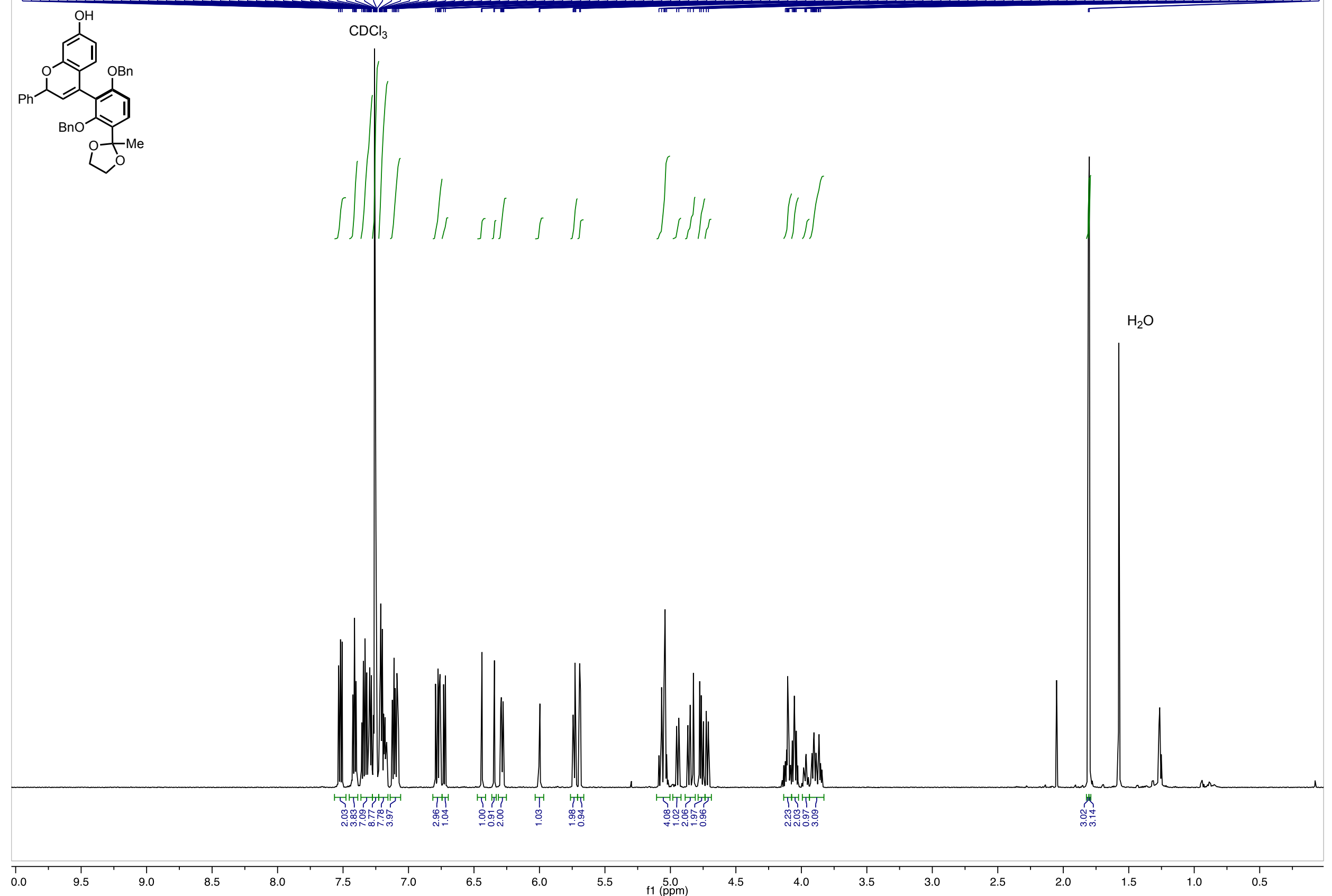

Figure S65. ${ }^{1} \mathrm{H}$ NMR $(600 \mathrm{MHz}$, chloroform- $d) 15$.

S121 


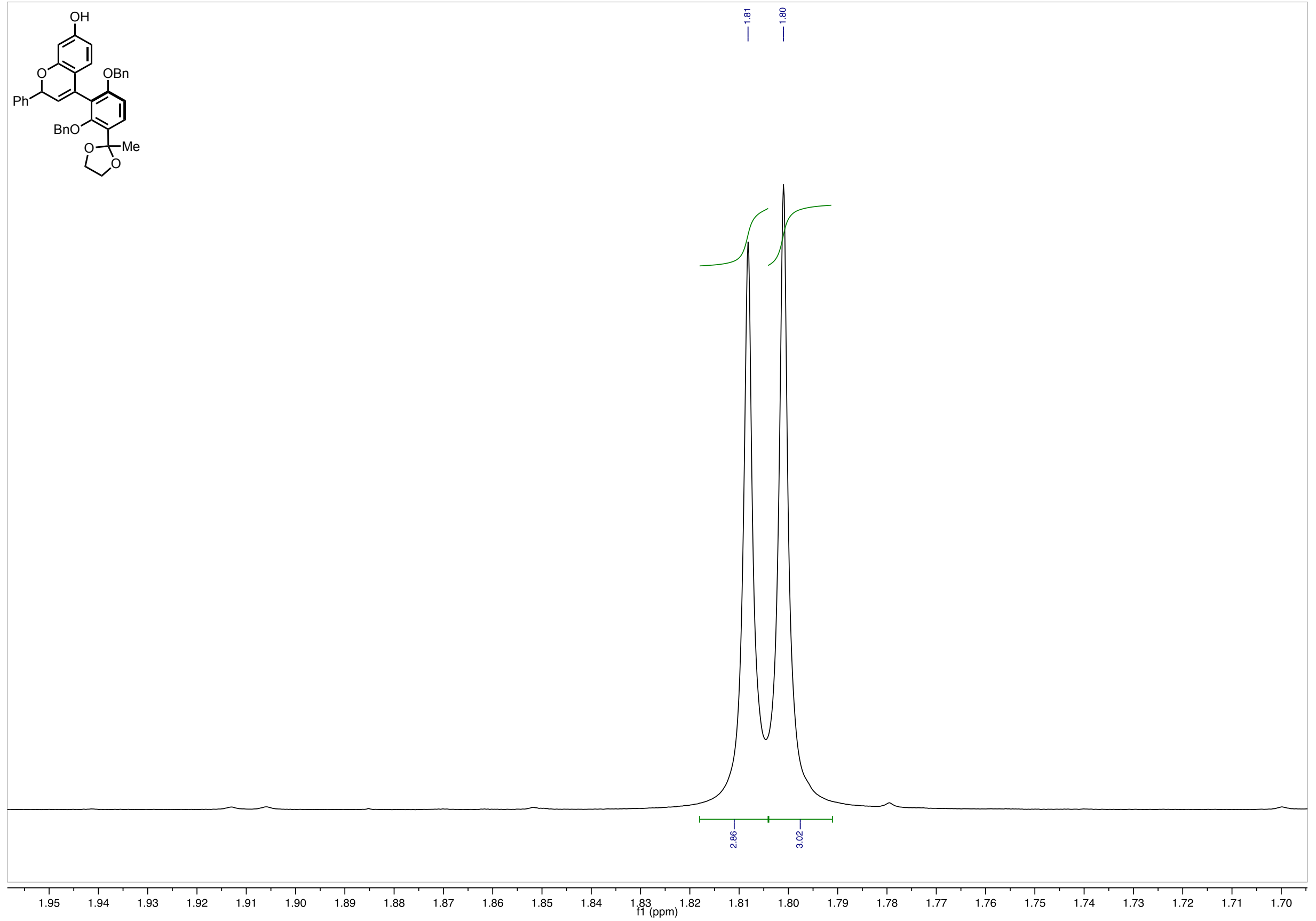

Figure S66. ${ }^{1} \mathrm{H}$ NMR (600 MHz, chloroform- $\left.d\right) 15$ (inset). 

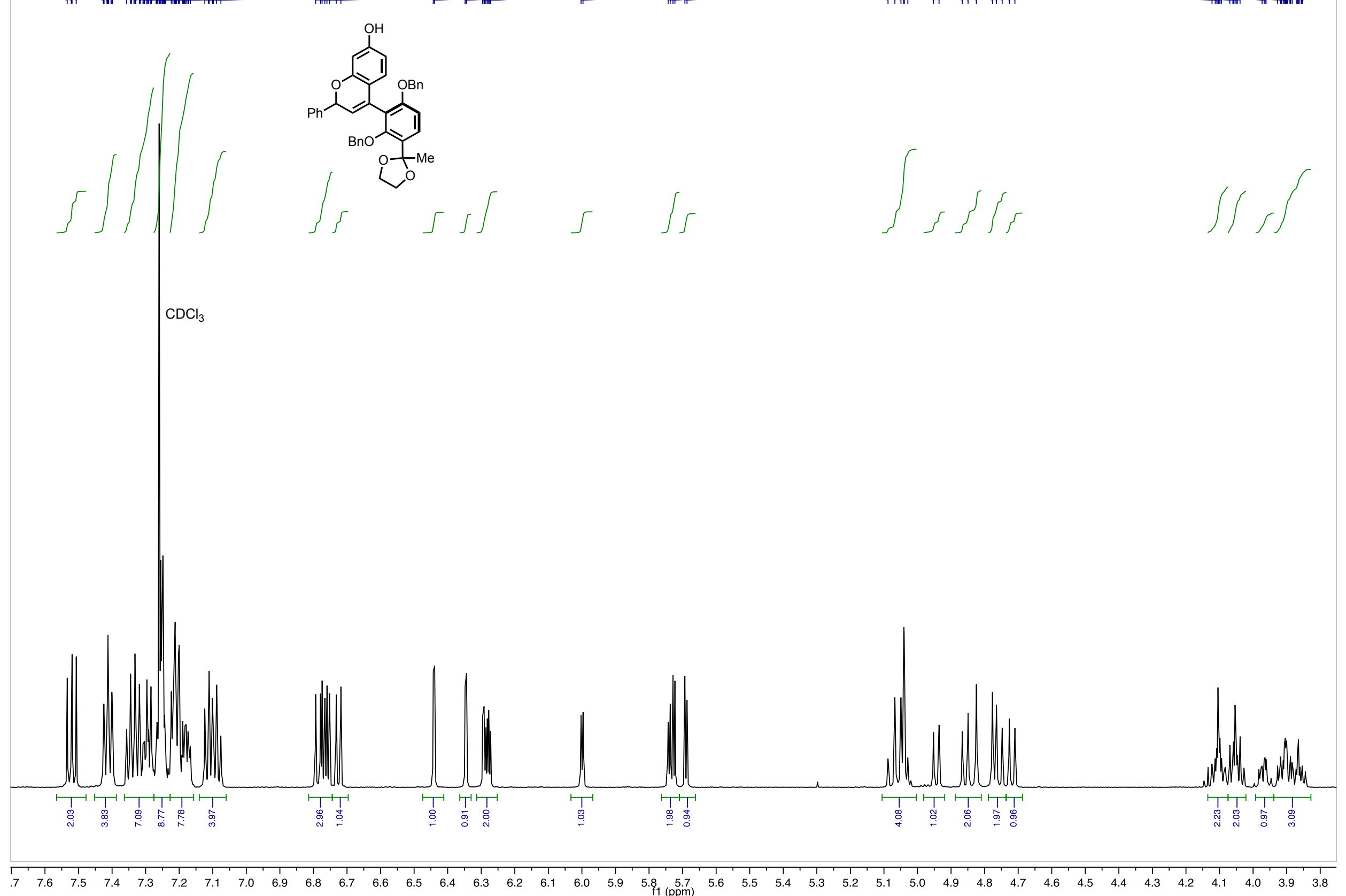

Figure S67. ${ }^{1} \mathrm{H}$ NMR (600 MHz, chloroform-d) 15 (inset). 


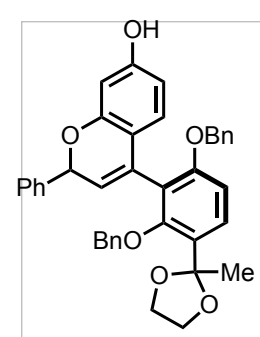

$\mathrm{CDCl}_{3}$
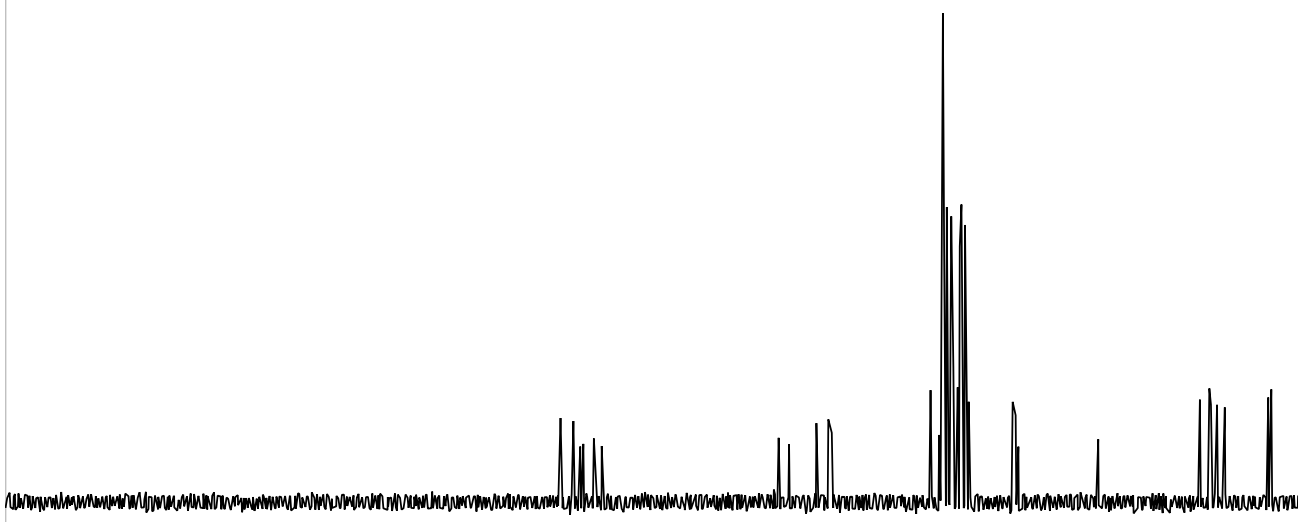

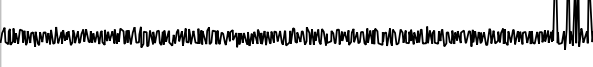
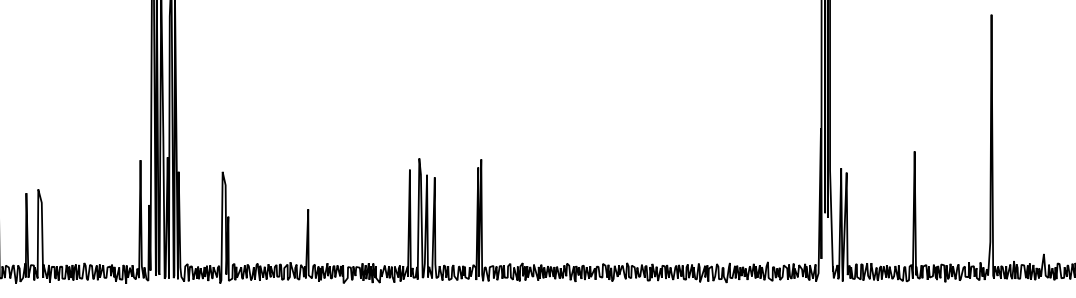

Figure S68. ${ }^{13} \mathrm{C}$ NMR (151 MHz, chloroform-d) 15. 

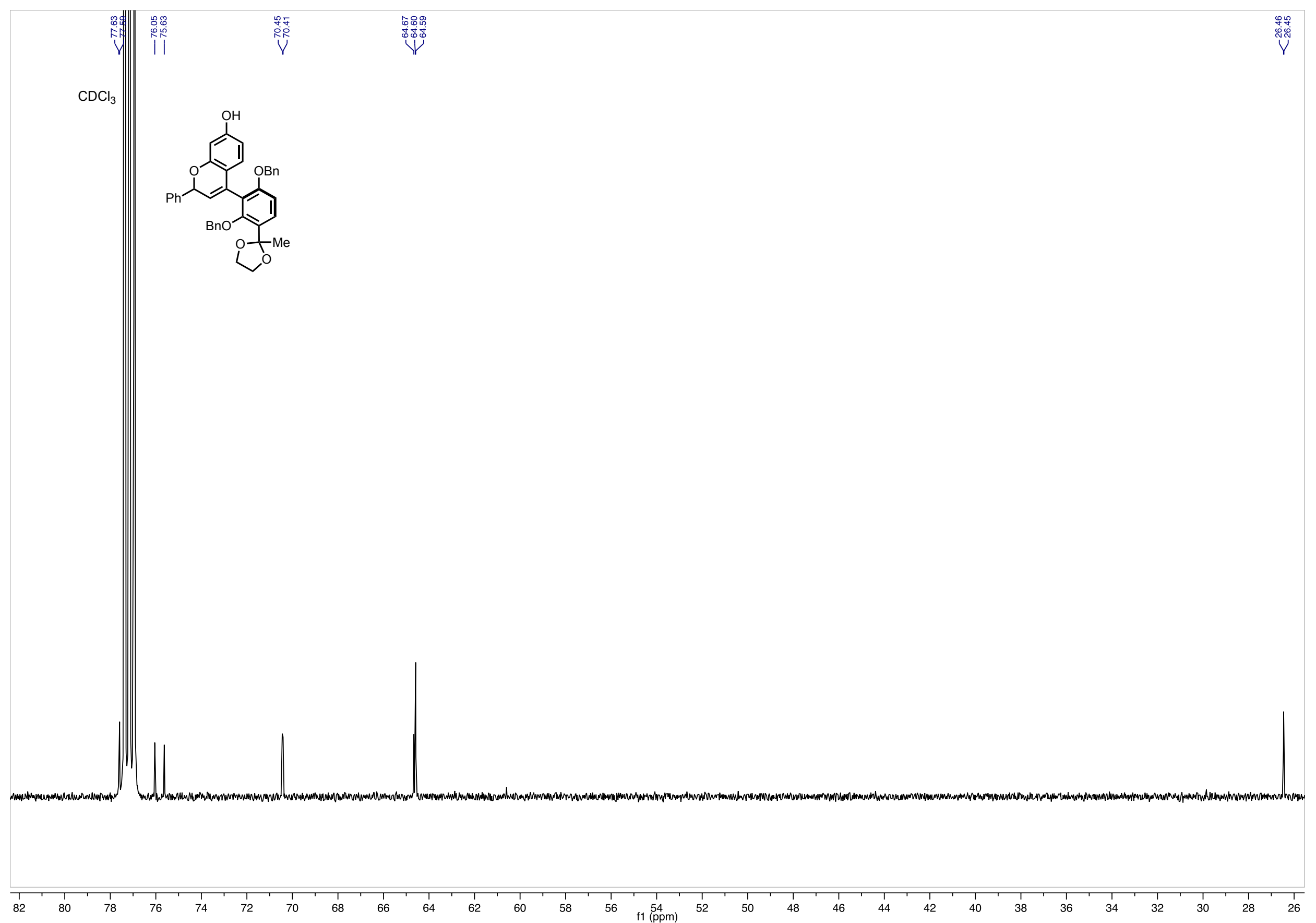

Figure S69. ${ }^{13} \mathrm{C}$ NMR (151 MHz, chloroform-d) 15 (inset).

S125 

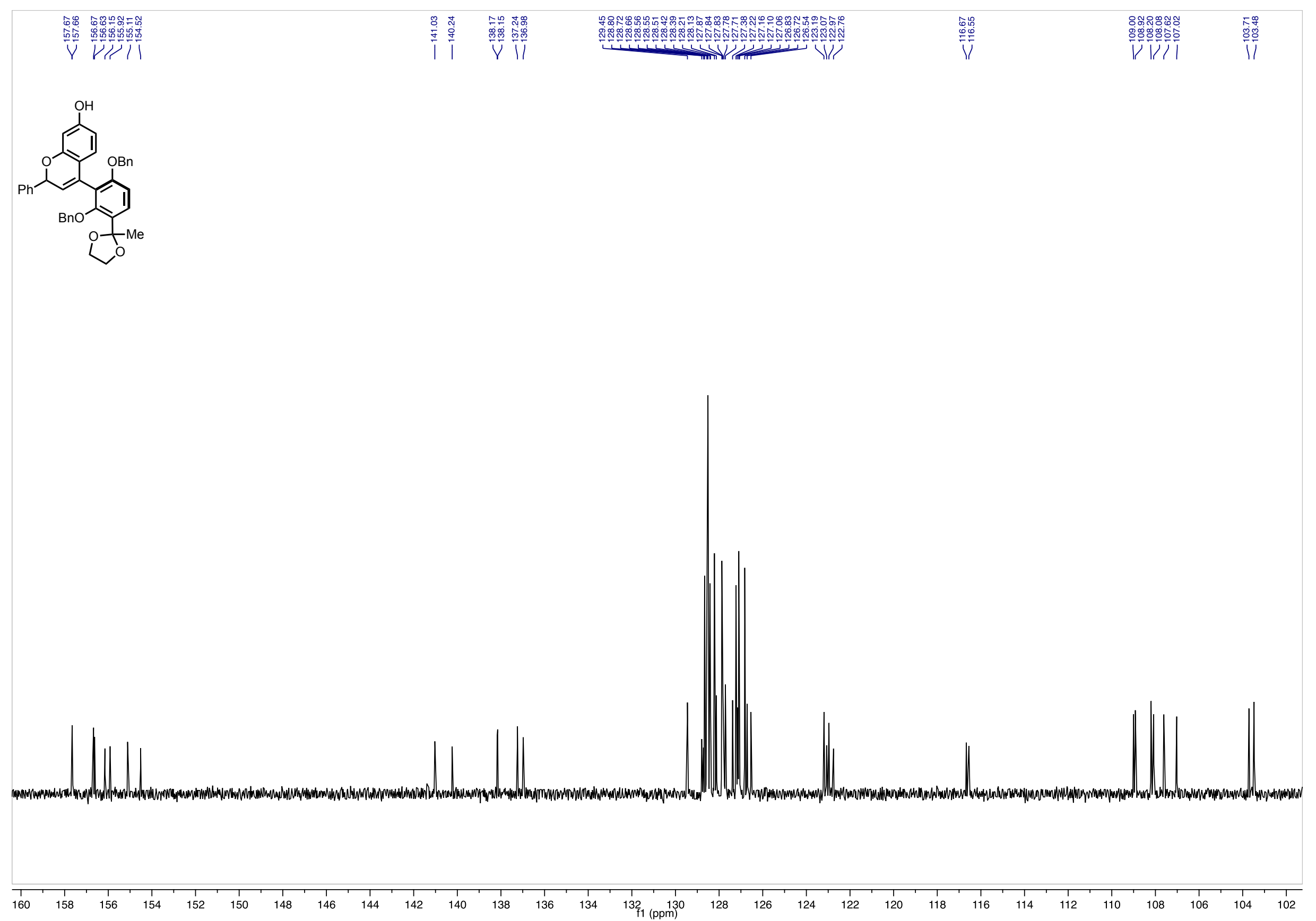

Figure S70. ${ }^{13} \mathrm{C}$ NMR ( $151 \mathrm{MHz}$, chloroform-d) 15 (inset). 

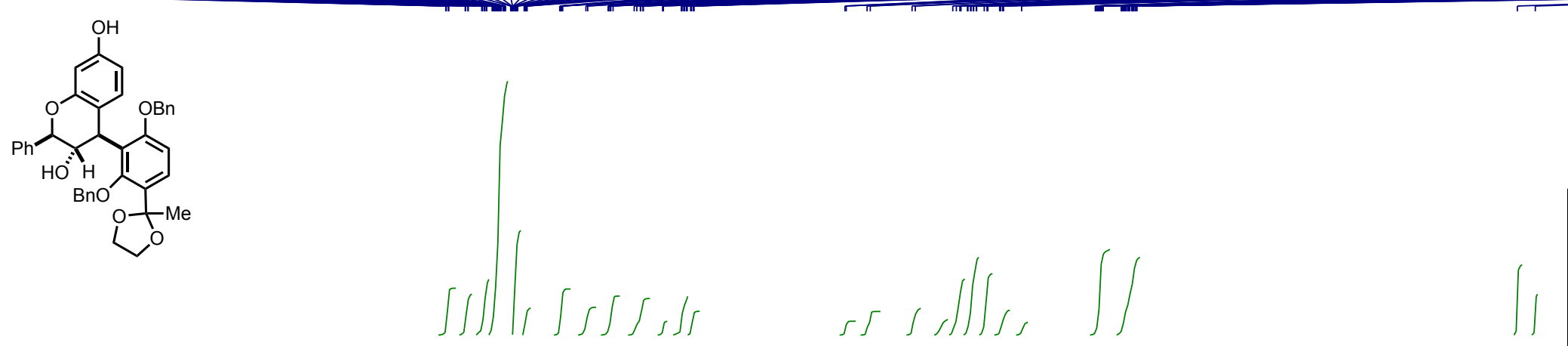

$\mathrm{CDCl}_{3}$
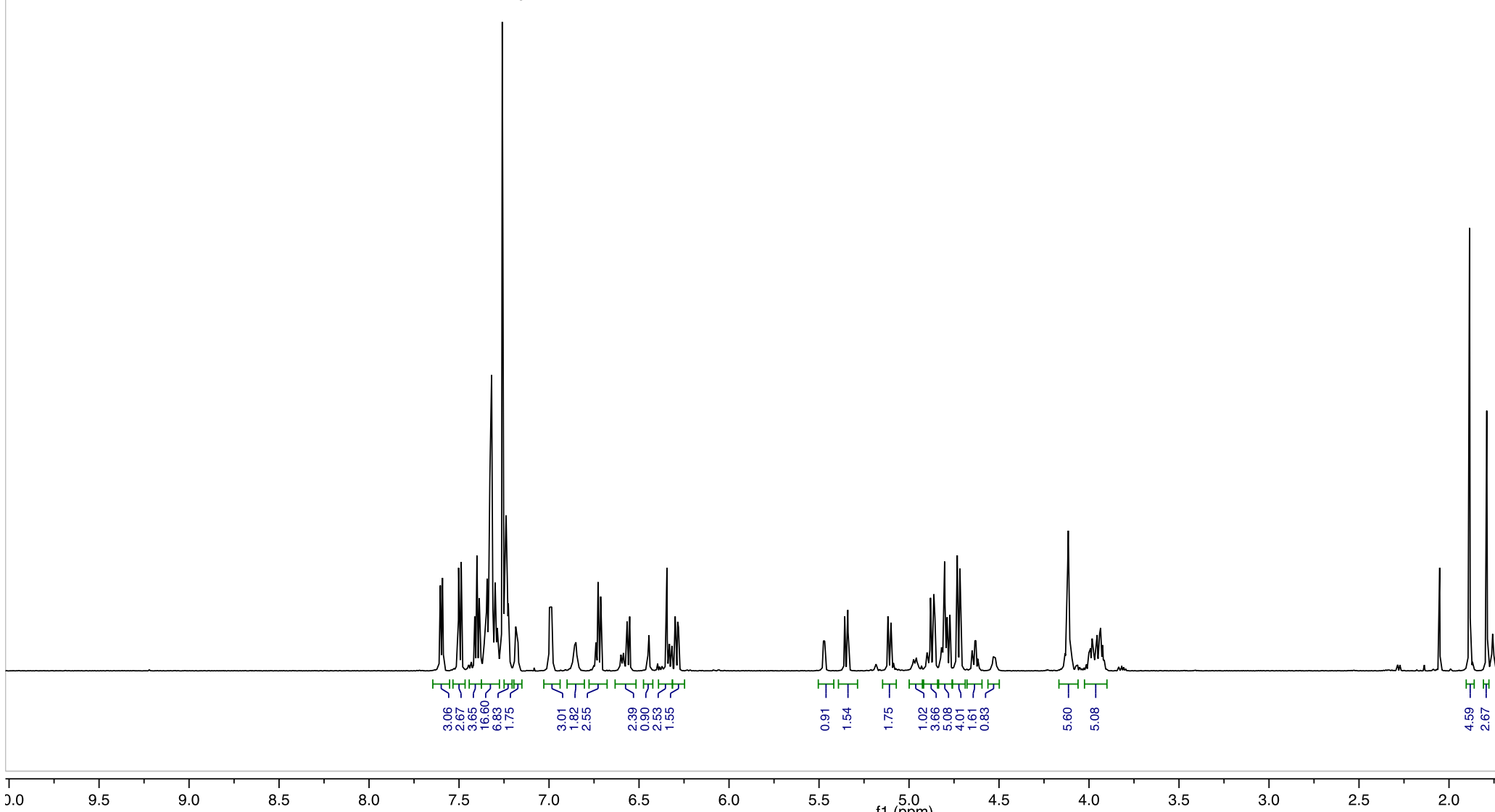

16 

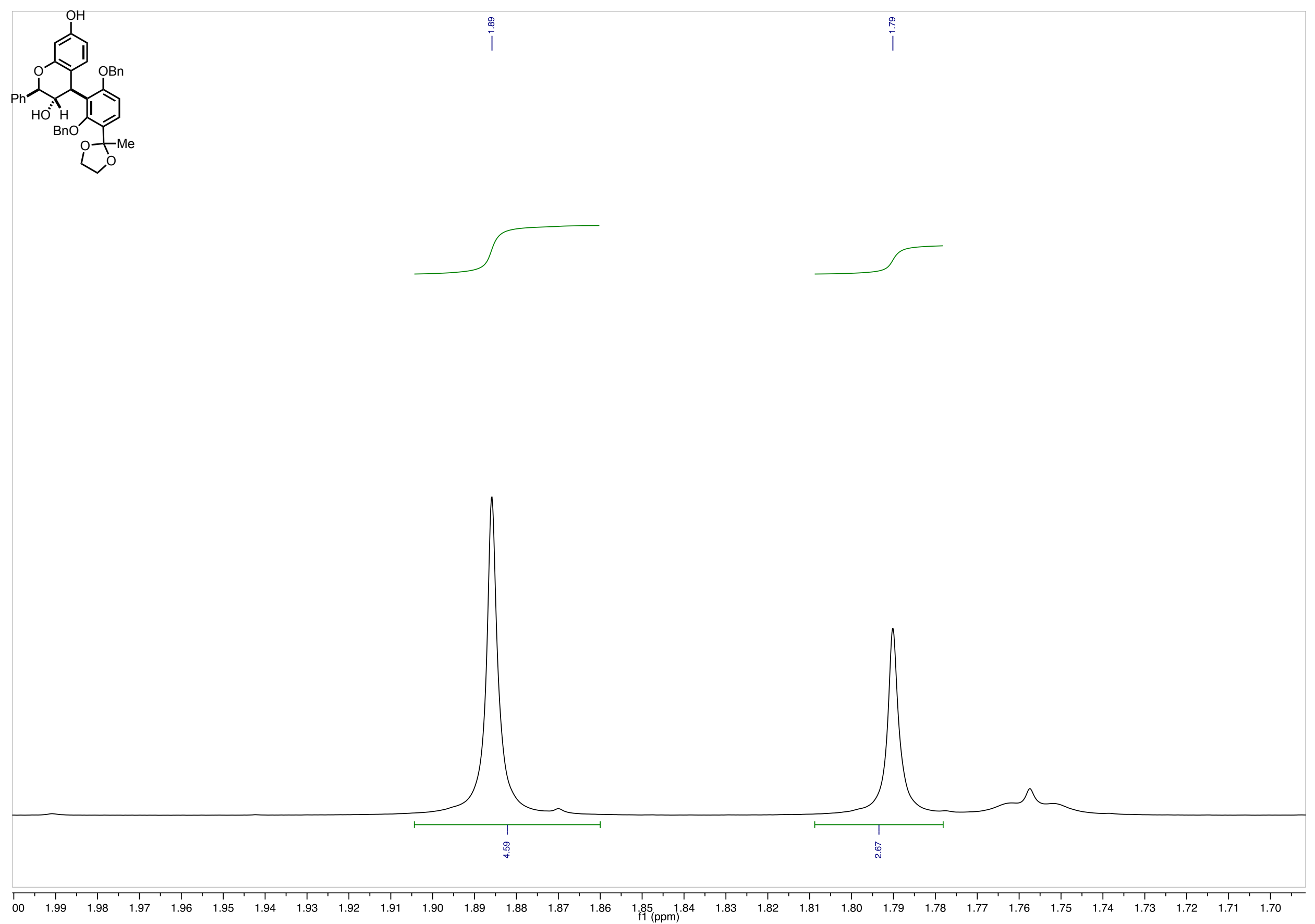

Figure S72. ${ }^{1} \mathrm{H}$ NMR (600 MHz, chloroform-d) 16 (inset).

$\mathrm{S} 128$ 

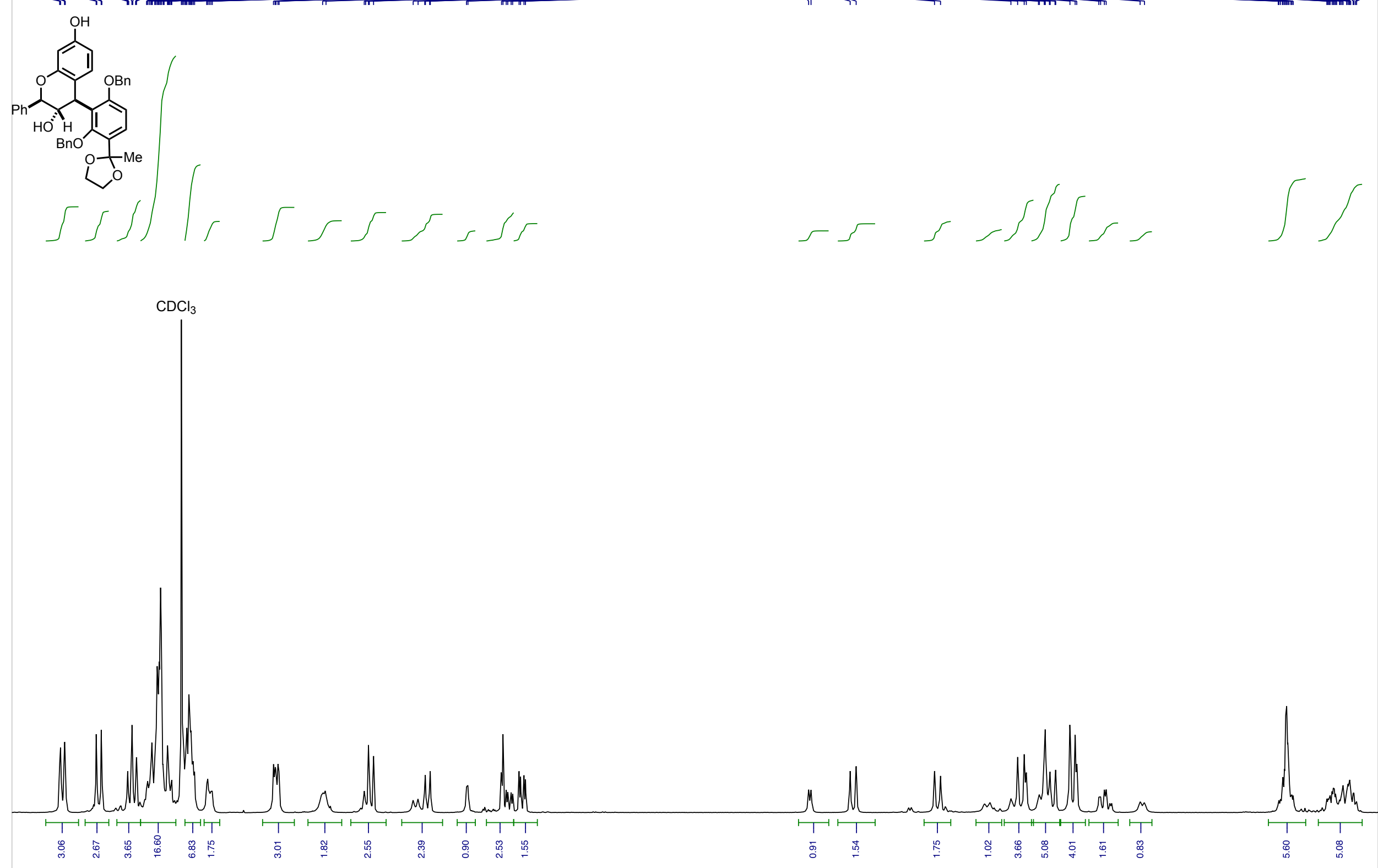

\begin{tabular}{ccccccc|cc}
\hline & 1 & 1 & 1 & 1 & \\
7.7 & 7.6 & 7.5 & 7.4 & 7.3 & 7.2
\end{tabular}

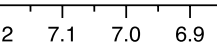
$\begin{array}{llll}6.8 & 6.7 & 6.6\end{array}$

Figure S73. ${ }^{1} \mathrm{H}$ NMR (600 MHz, chloroform-d) 16 (inset). 

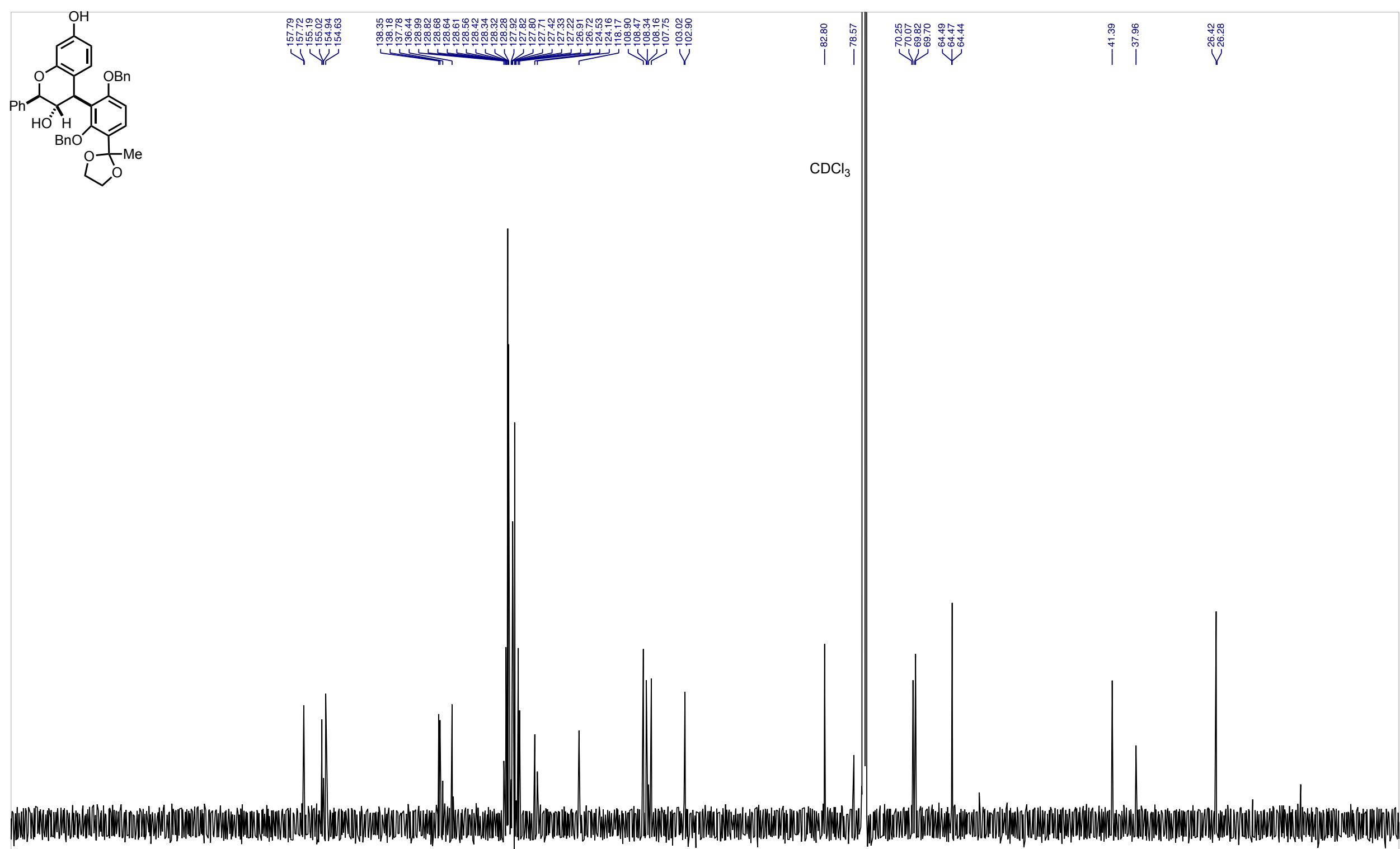

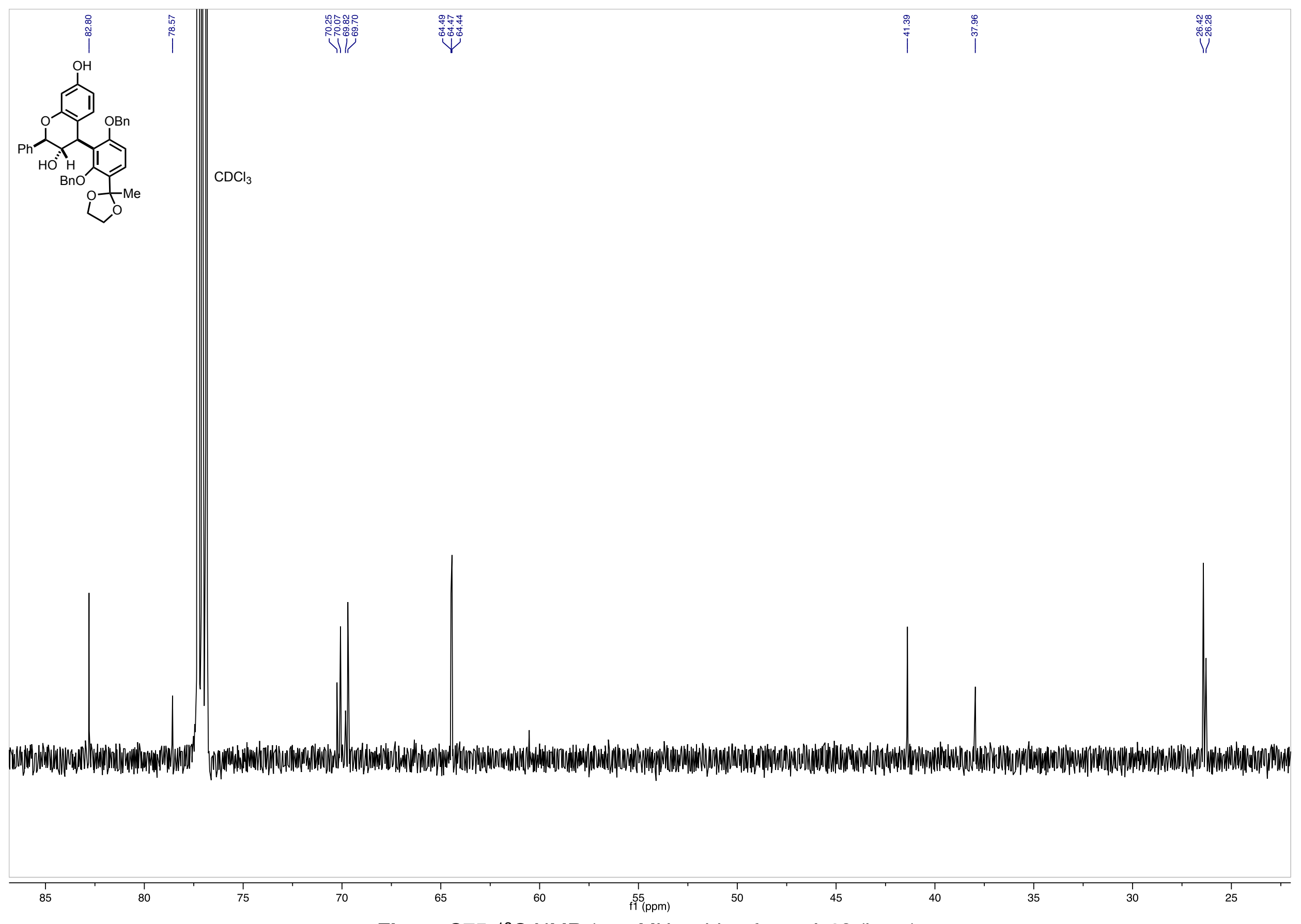

Figure S75. ${ }^{13} \mathrm{C}$ NMR (151 MHz, chloroform- $d$ ) 16 (inset).

S131 


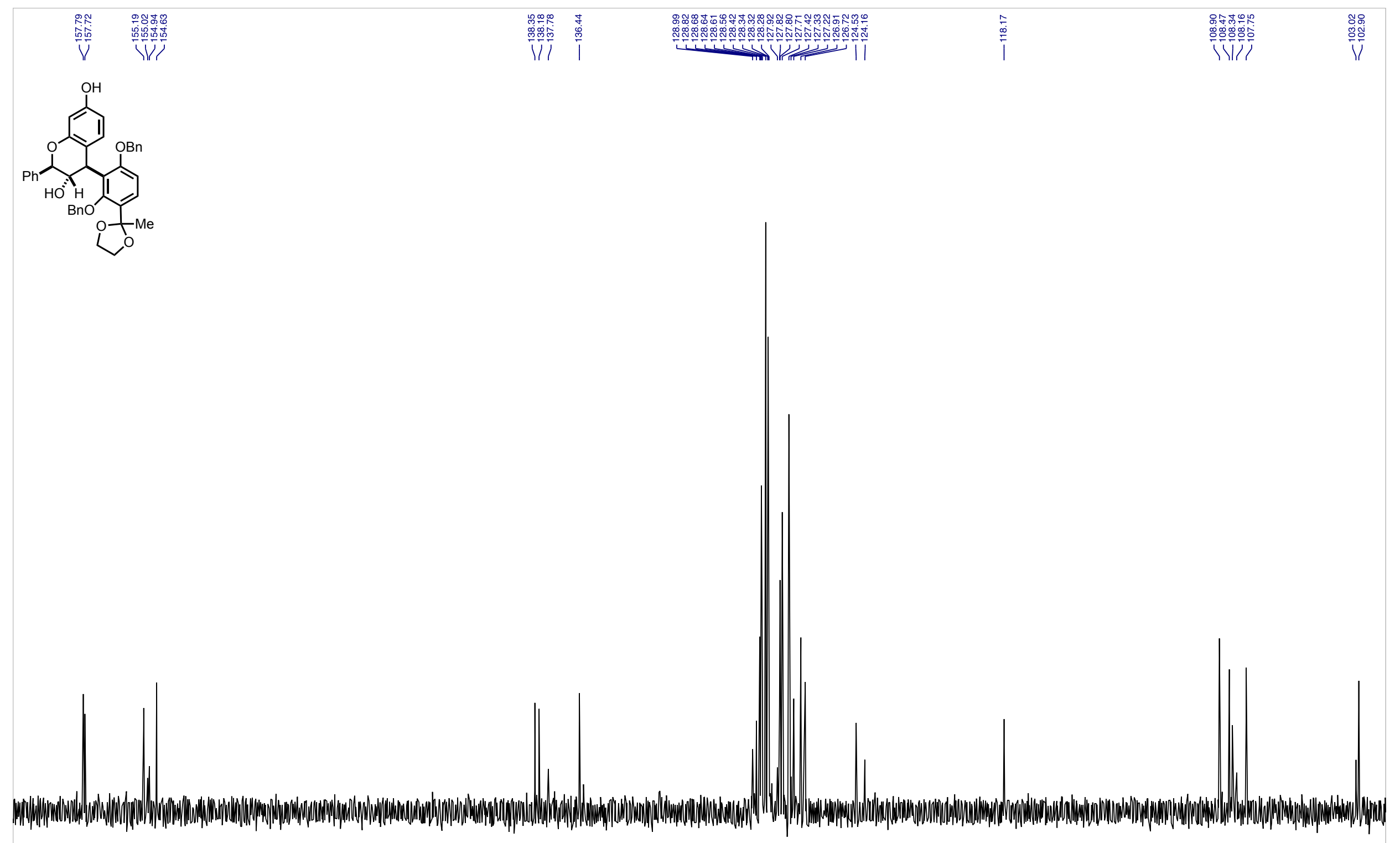

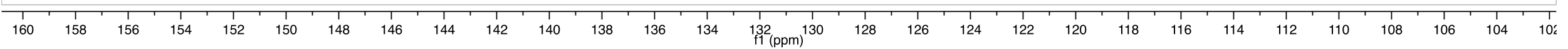

Figure S76. ${ }^{13} \mathrm{C}$ NMR ( $151 \mathrm{MHz}$, chloroform- $d$ ) 16 (inset). 

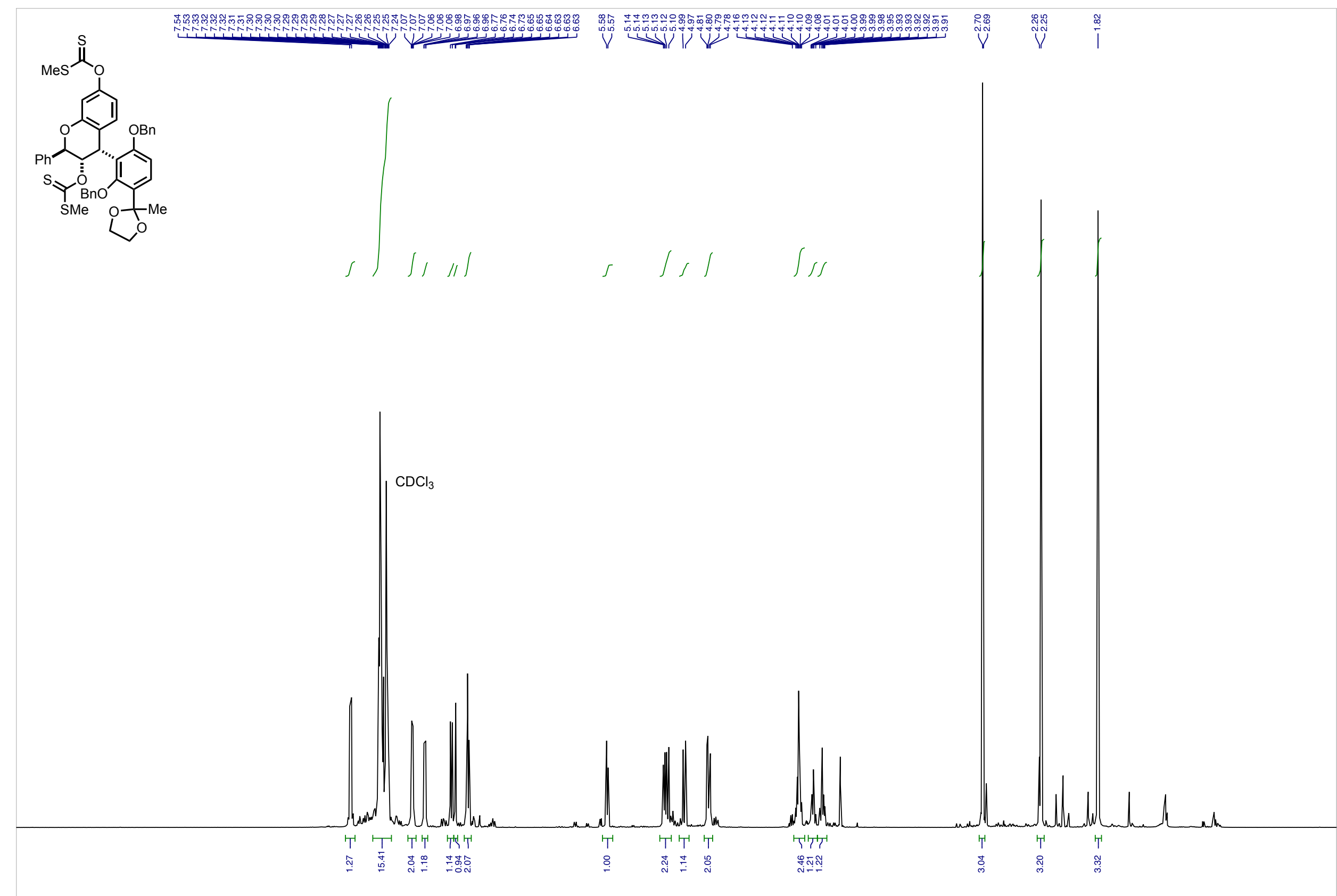

Figure S77. ${ }^{1 H}$ NMR (600 MHz, chloroform- $d$ ) 17. 


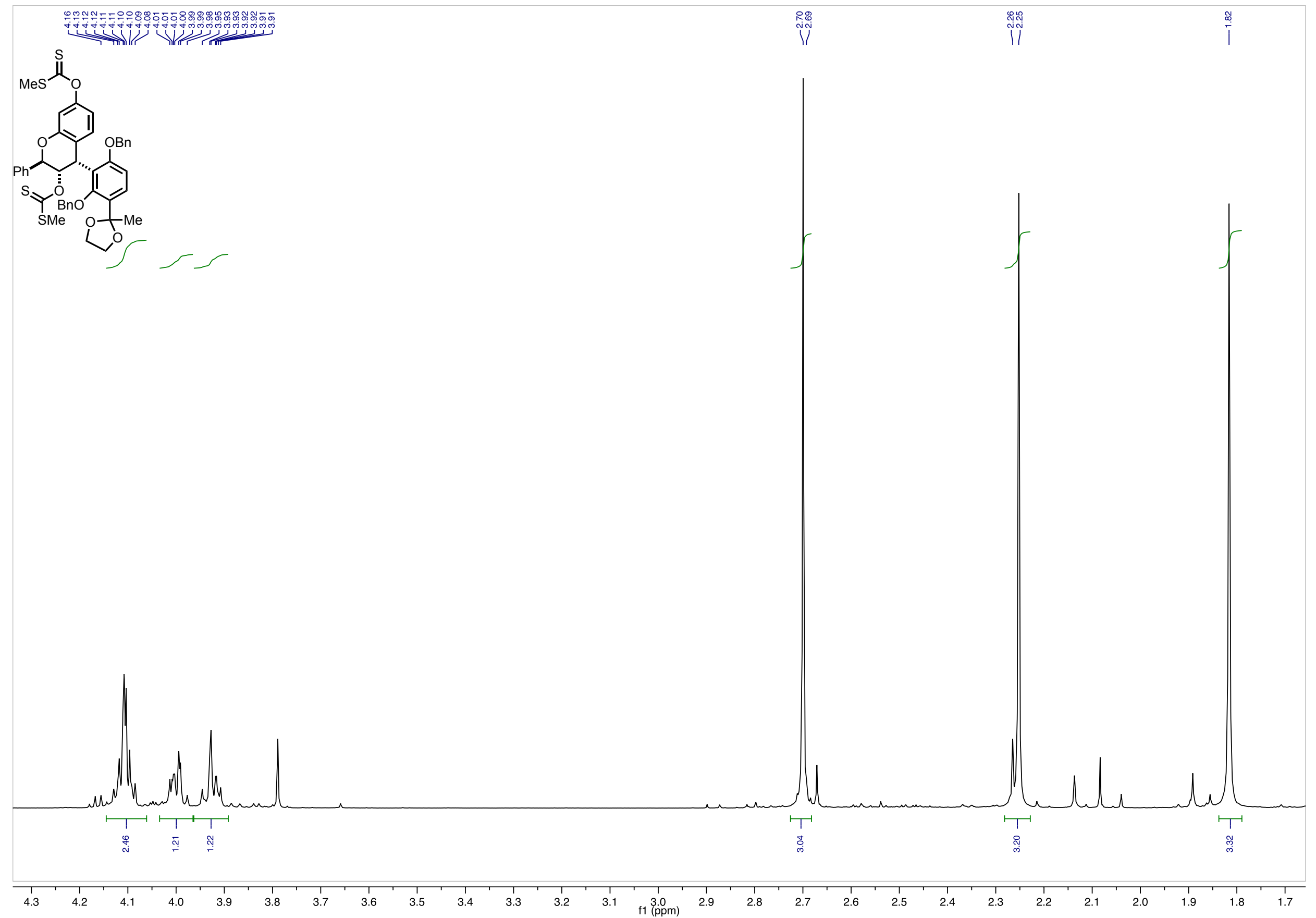

Figure S78. ${ }^{1} \mathrm{H}$ NMR (600 MHz, chloroform-d) 17 (inset). 

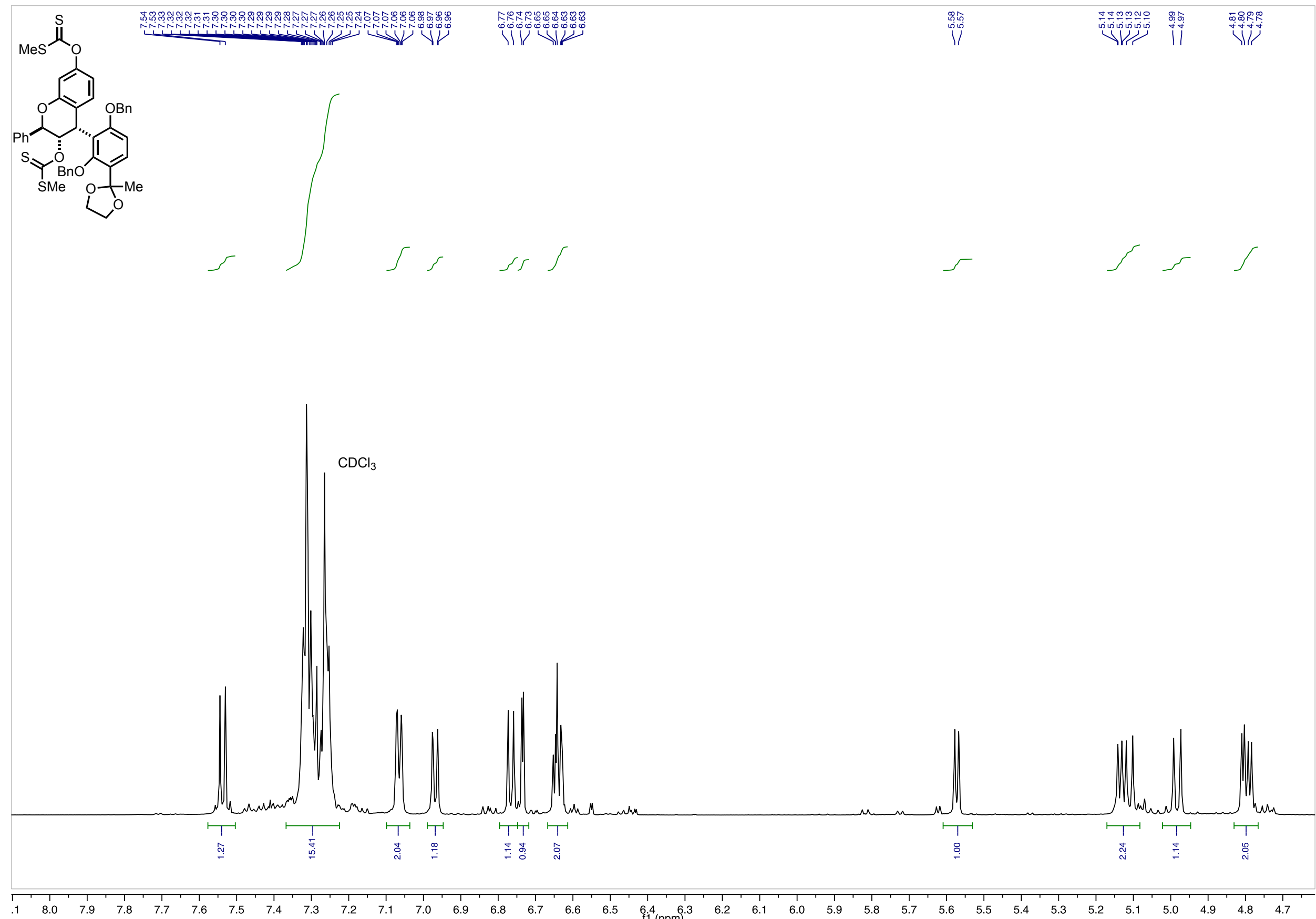
$7.3 \quad 7.2$

Figure S79. ${ }^{1} \mathrm{H}$ NMR (600 MHz, chloroform- $d$ ) 17 (inset). 

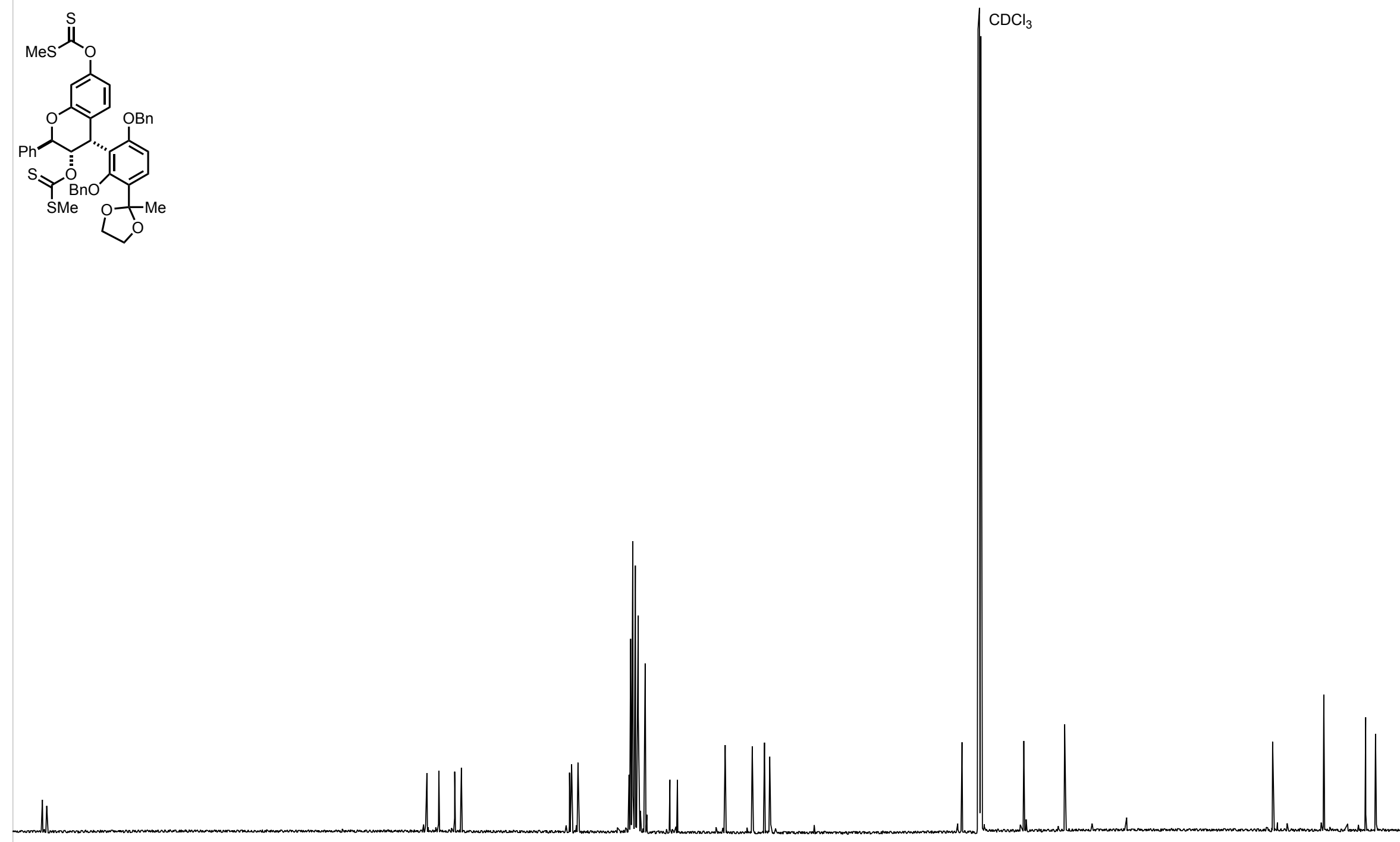

Figure S80. ${ }^{13} \mathrm{C}$ NMR (151 MHz, chloroform-d) 17. 

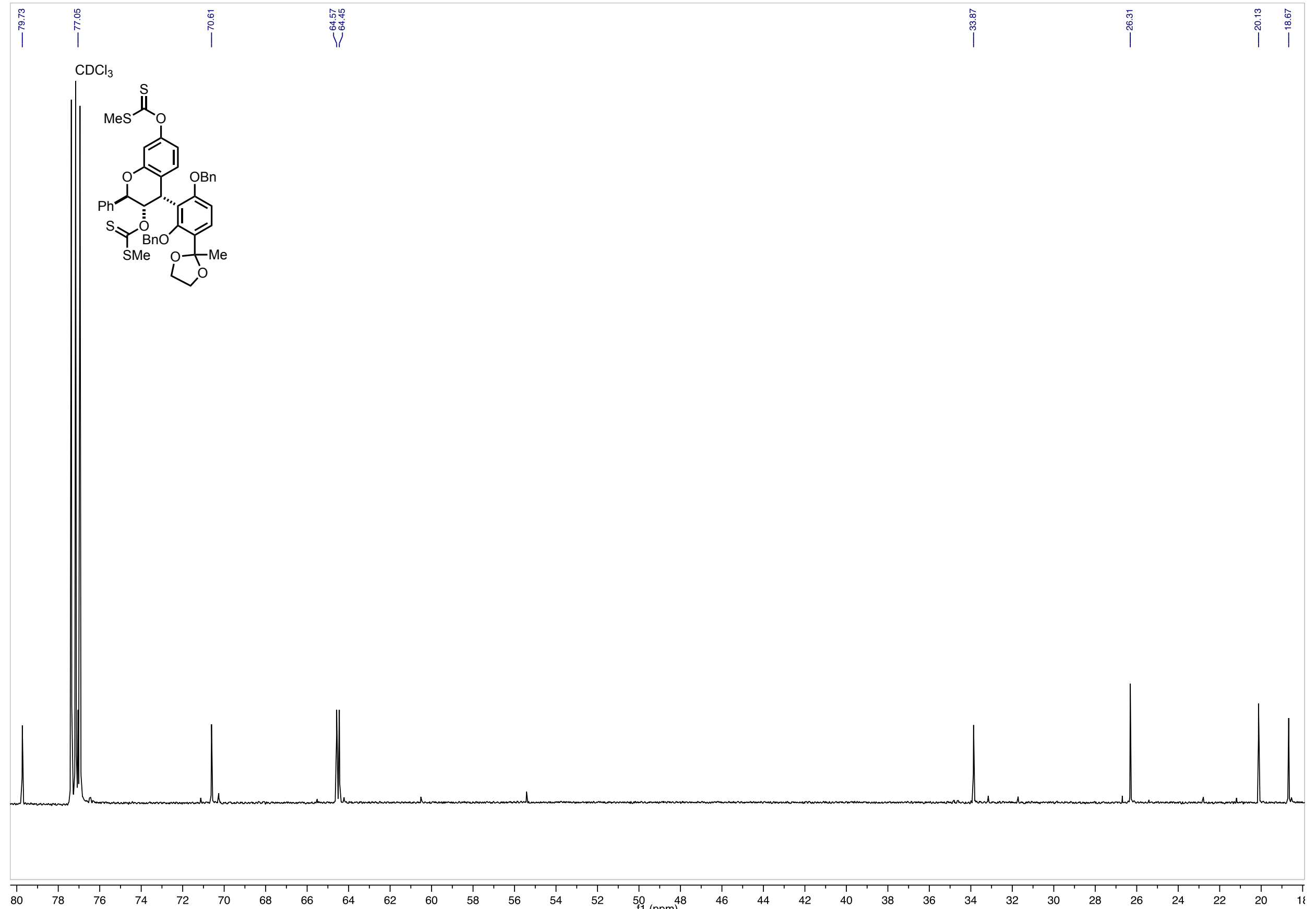

Figure S81. ${ }^{13} \mathrm{C}$ NMR (151 MHz, chloroform- $d$ ) 17 (inset). 

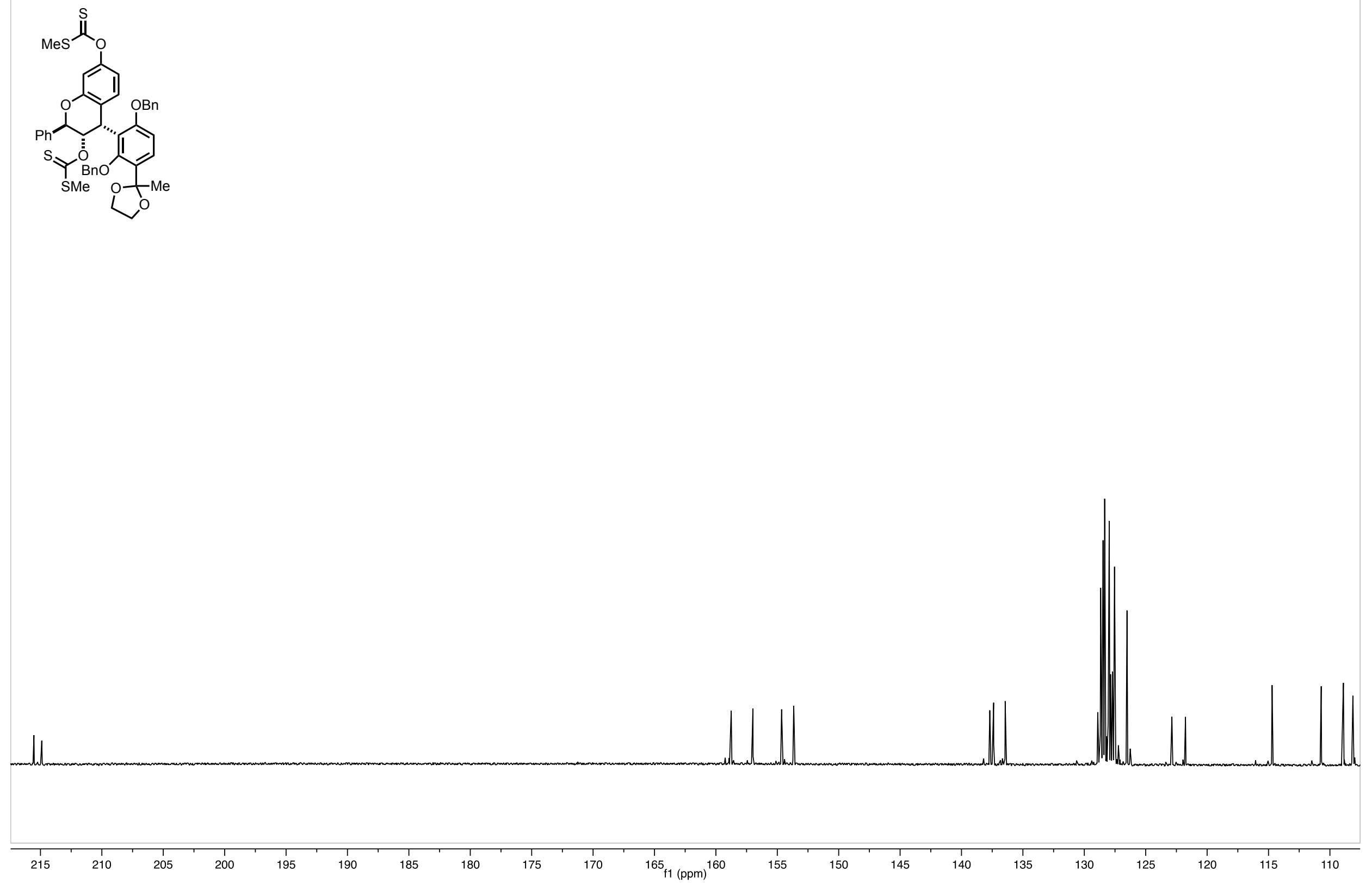

Figure S82. ${ }^{13} \mathrm{C}$ NMR (151 MHz, chloroform- $d$ ) 17 (inset).

S138 

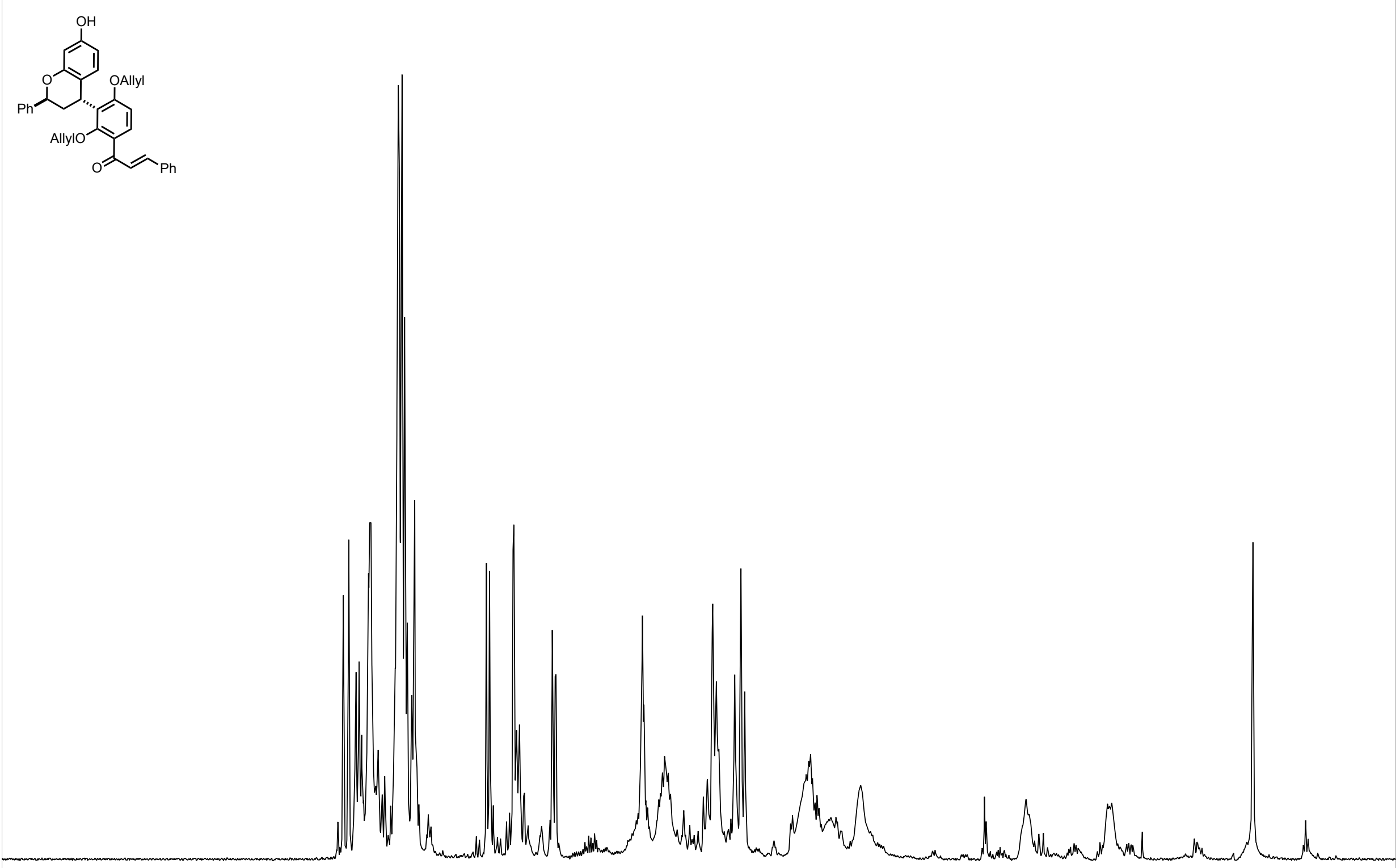

10.0

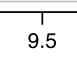




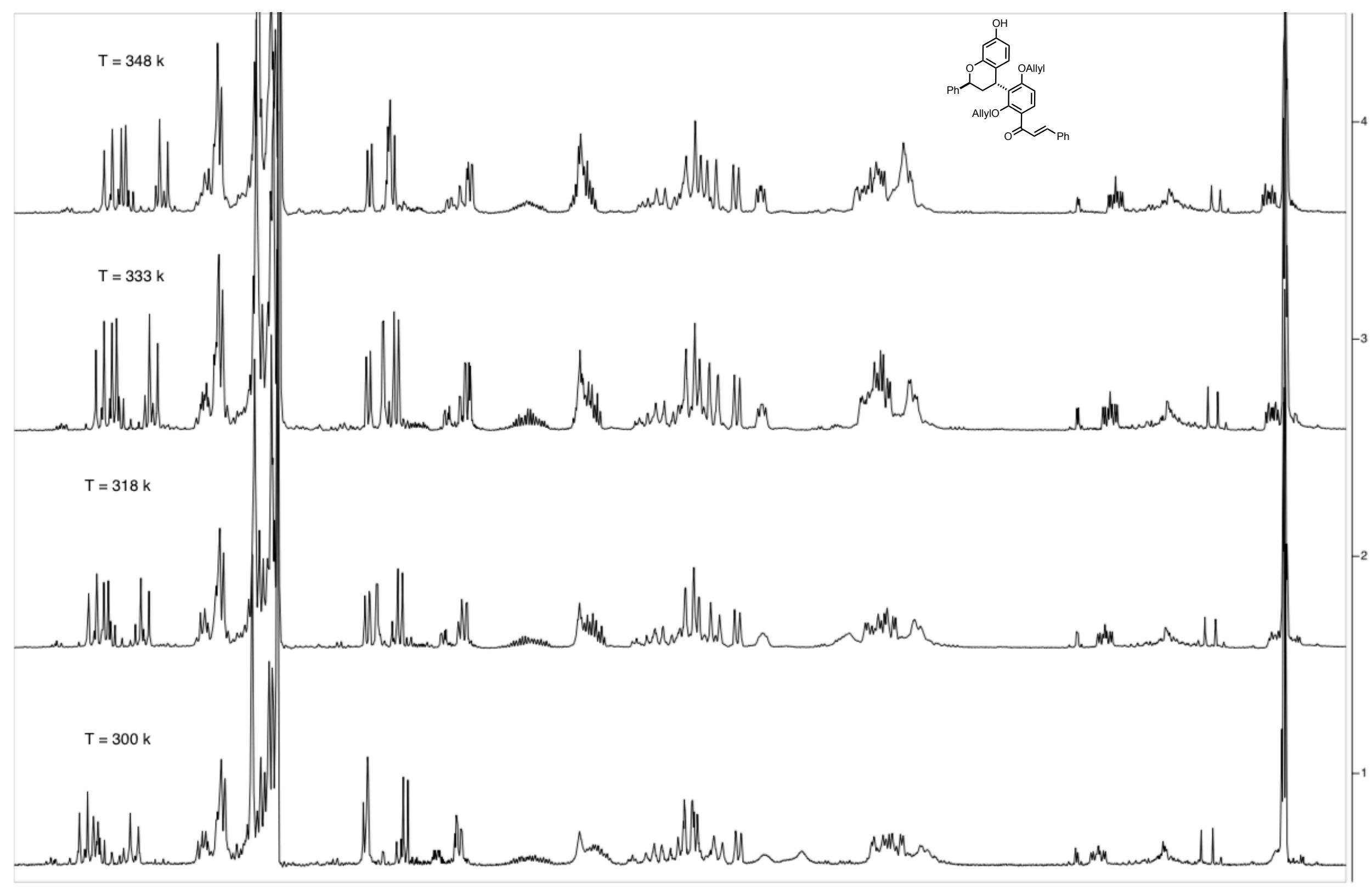

8.0

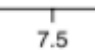

7.0

6.5

6.0

${ }_{5.5}^{1}$

f1 (ppm)

4.5

4.0

3.5

3.0

2.5

2.0

Figure S84. Variable temperature ${ }^{1} \mathrm{H}$ NMR of $18 \mathrm{a}\left(400 \mathrm{MHz}, \mathrm{PhMe}-\mathrm{d}_{8}\right)$ from 300 to $348 \mathrm{k}$.

S140 


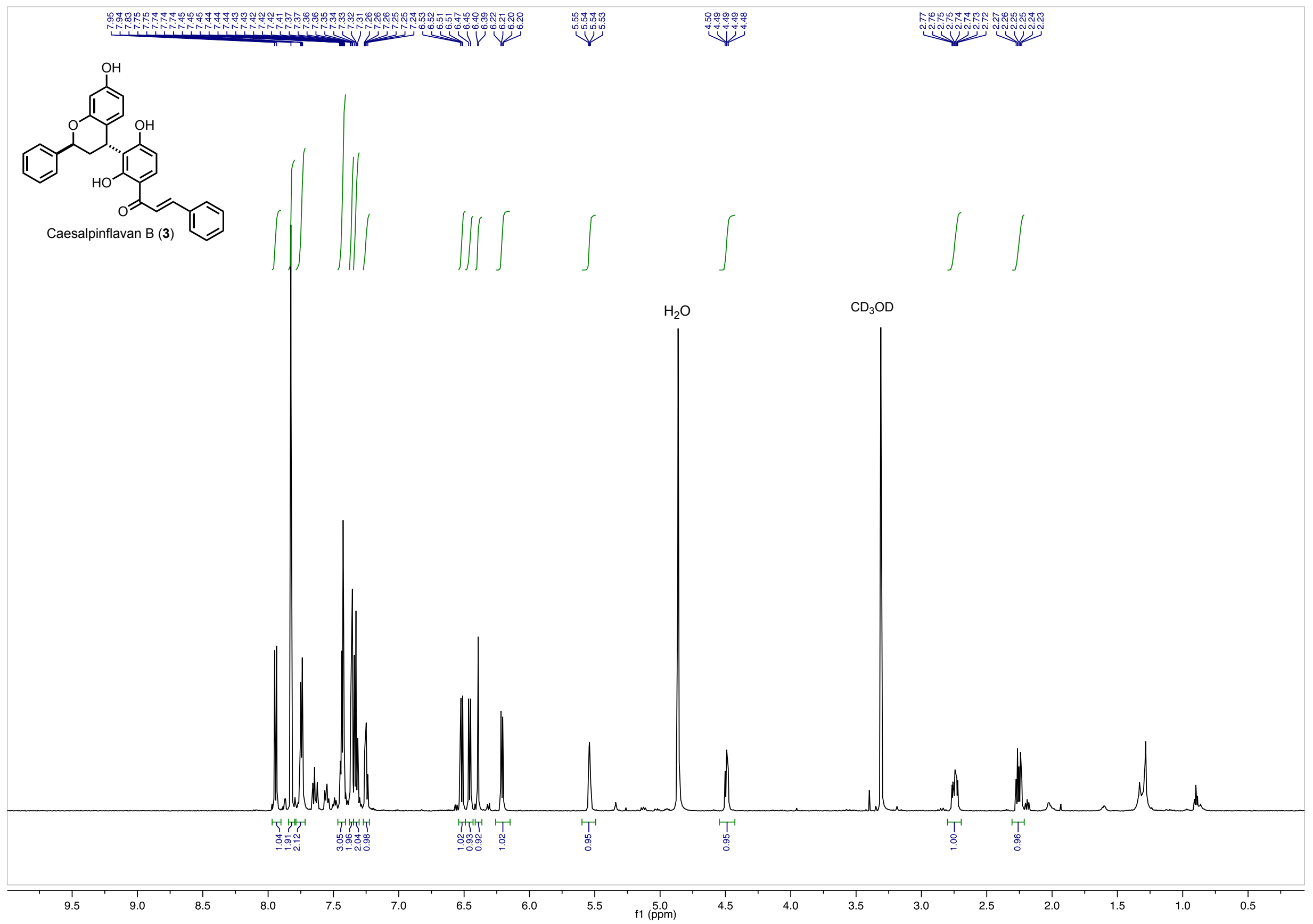

Figure S85. ${ }^{1} \mathrm{H}$ NMR (600 MHz, methanol- $\left.d_{4}\right)$, Caesalpinflavan B (3). 


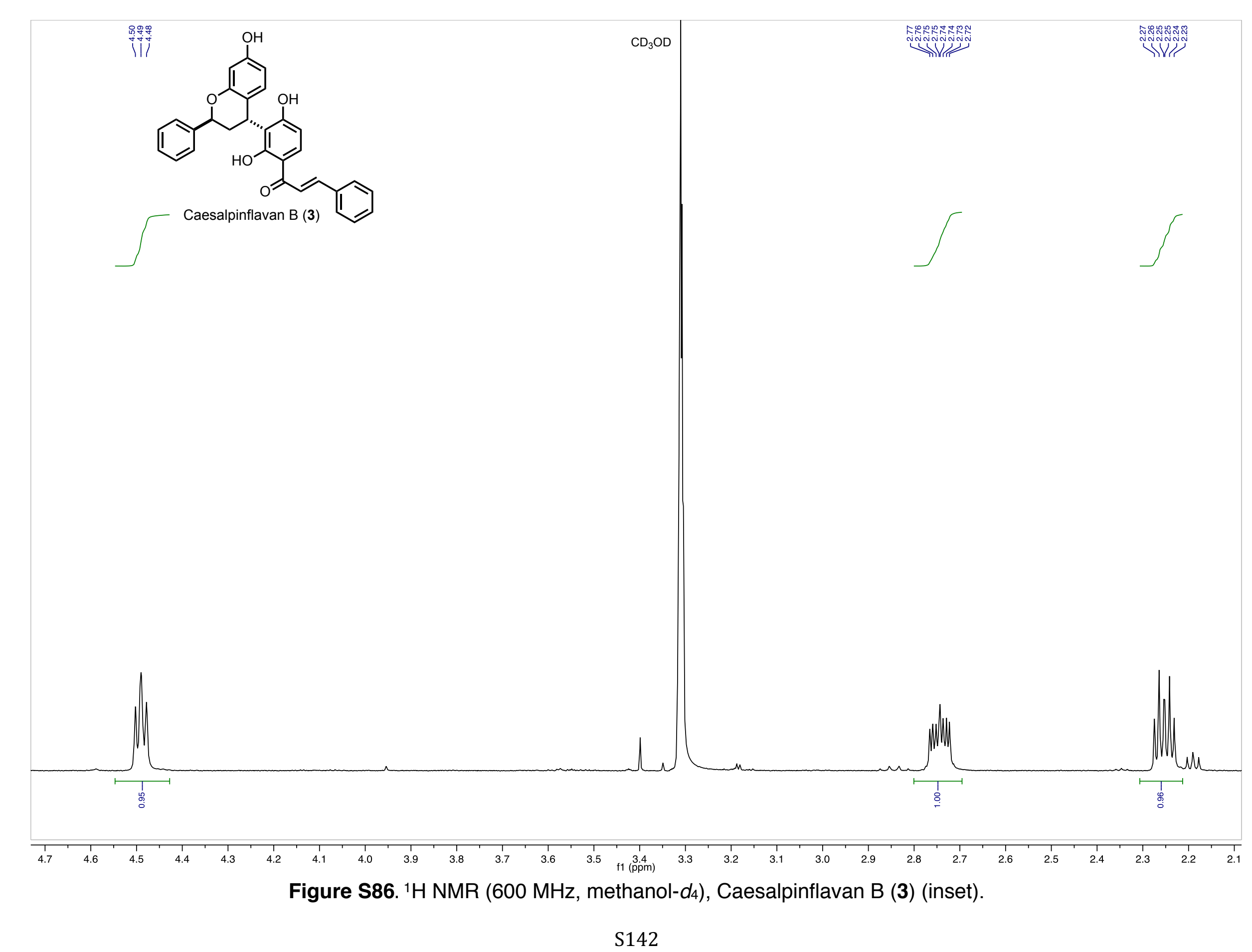




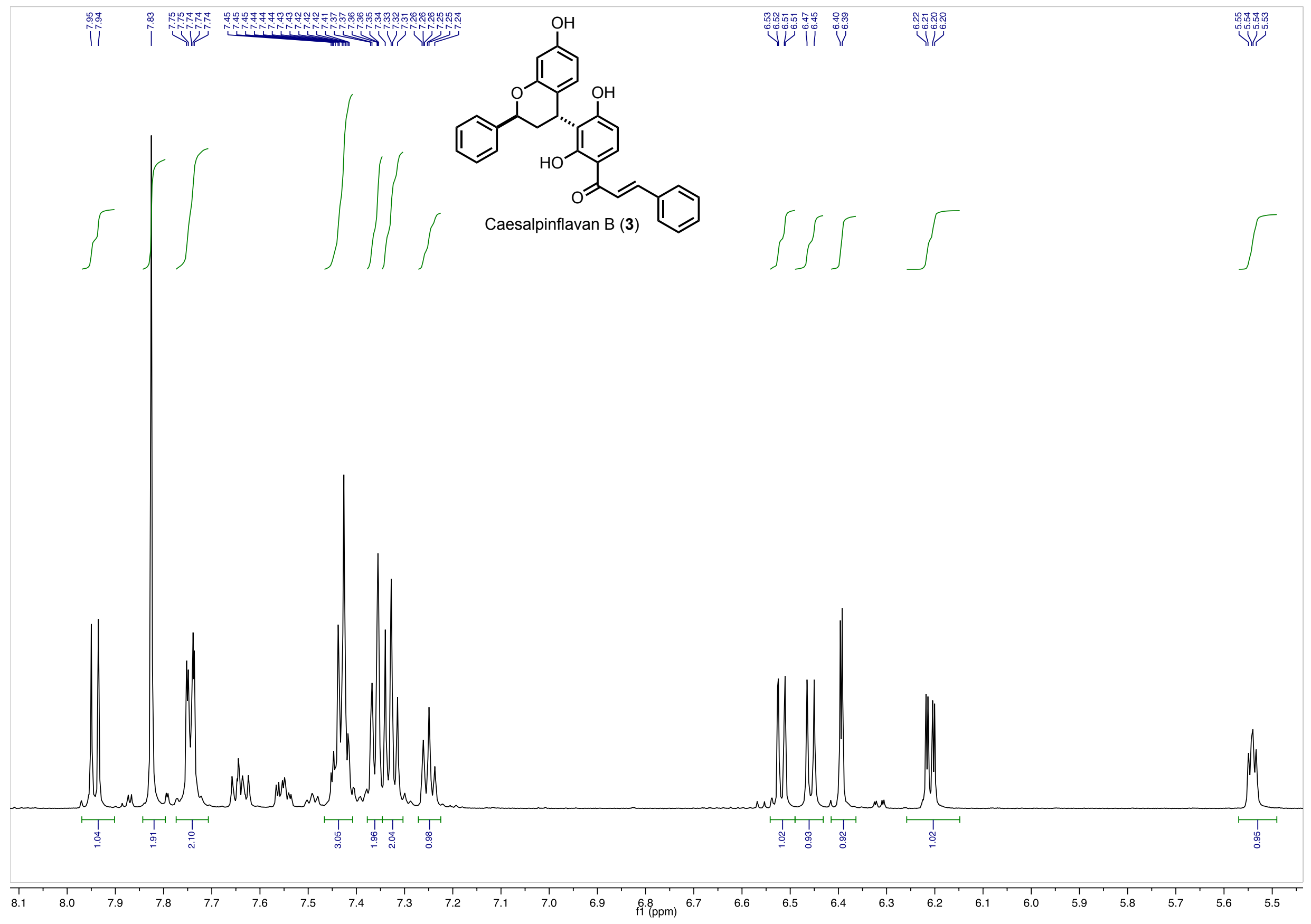

Figure $\mathbf{S 8 7}{ }^{1} \mathrm{H}$ NMR $\left(600 \mathrm{MHz}\right.$, methanol- $\left.d_{4}\right)$, Caesalpinflavan B (3) (inset). 


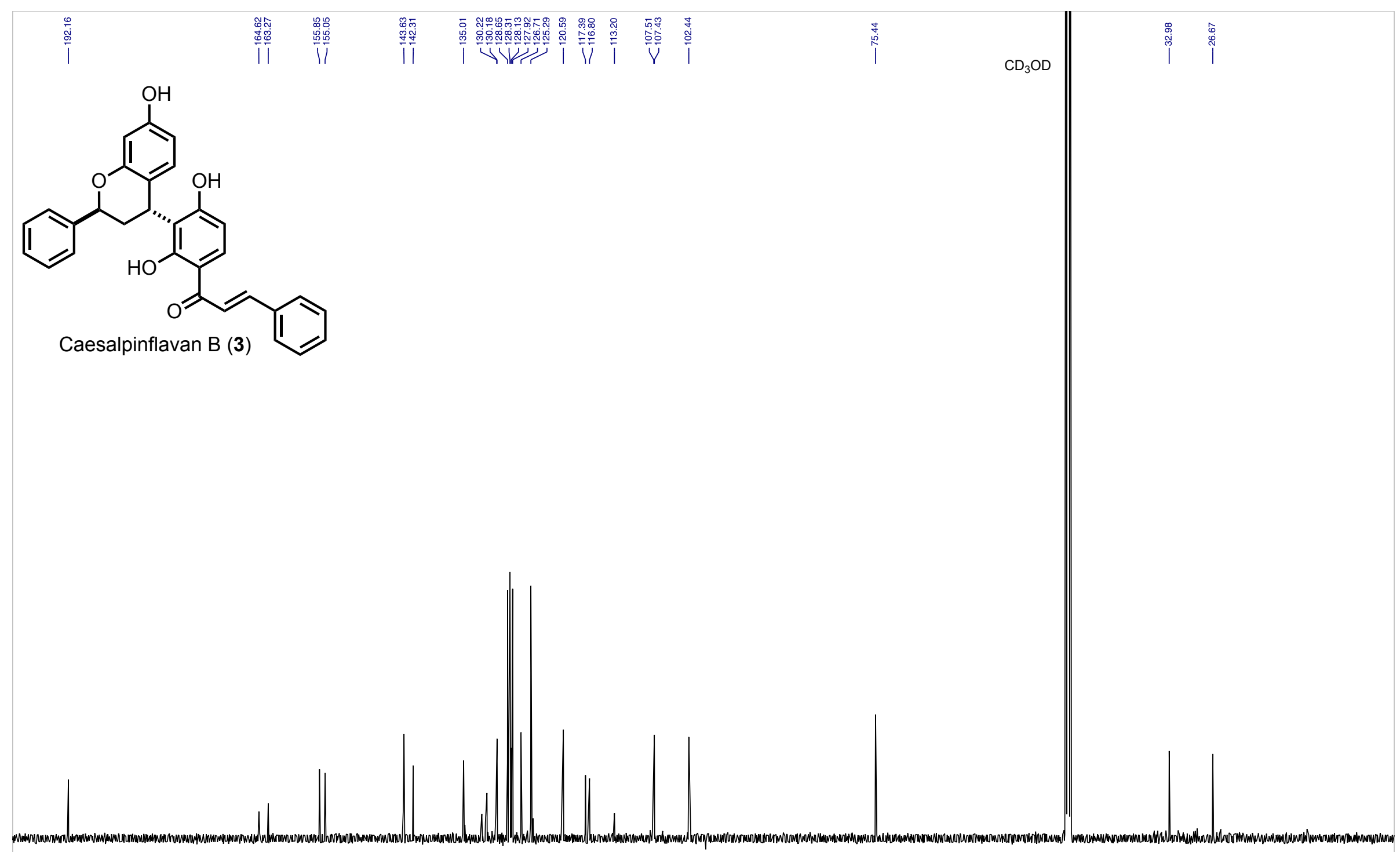

$190 \quad 180$

170

160

Figure S88. ${ }^{13} \mathrm{C}$ NMR $\left(151 \mathrm{MHz}\right.$, methanol- $\left.d_{4}\right)$, Caesalpinflavan $\mathrm{B}(\mathbf{3})$ 


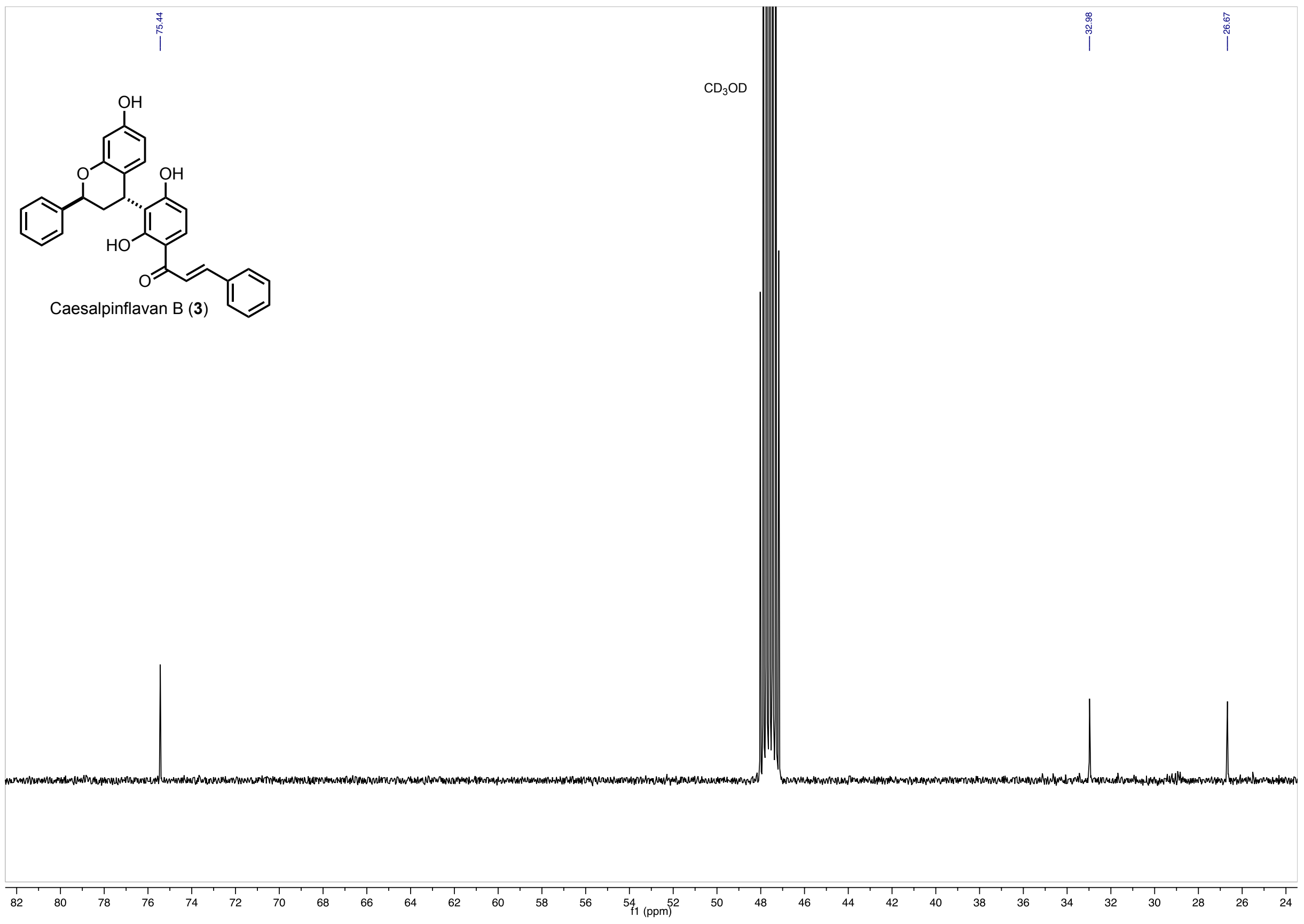

Figure S89. ${ }^{13} \mathrm{C}$ NMR (151 MHz, methanol- $\left.d_{4}\right)$, Caesalpinflavan B (3) (inset).

S145 


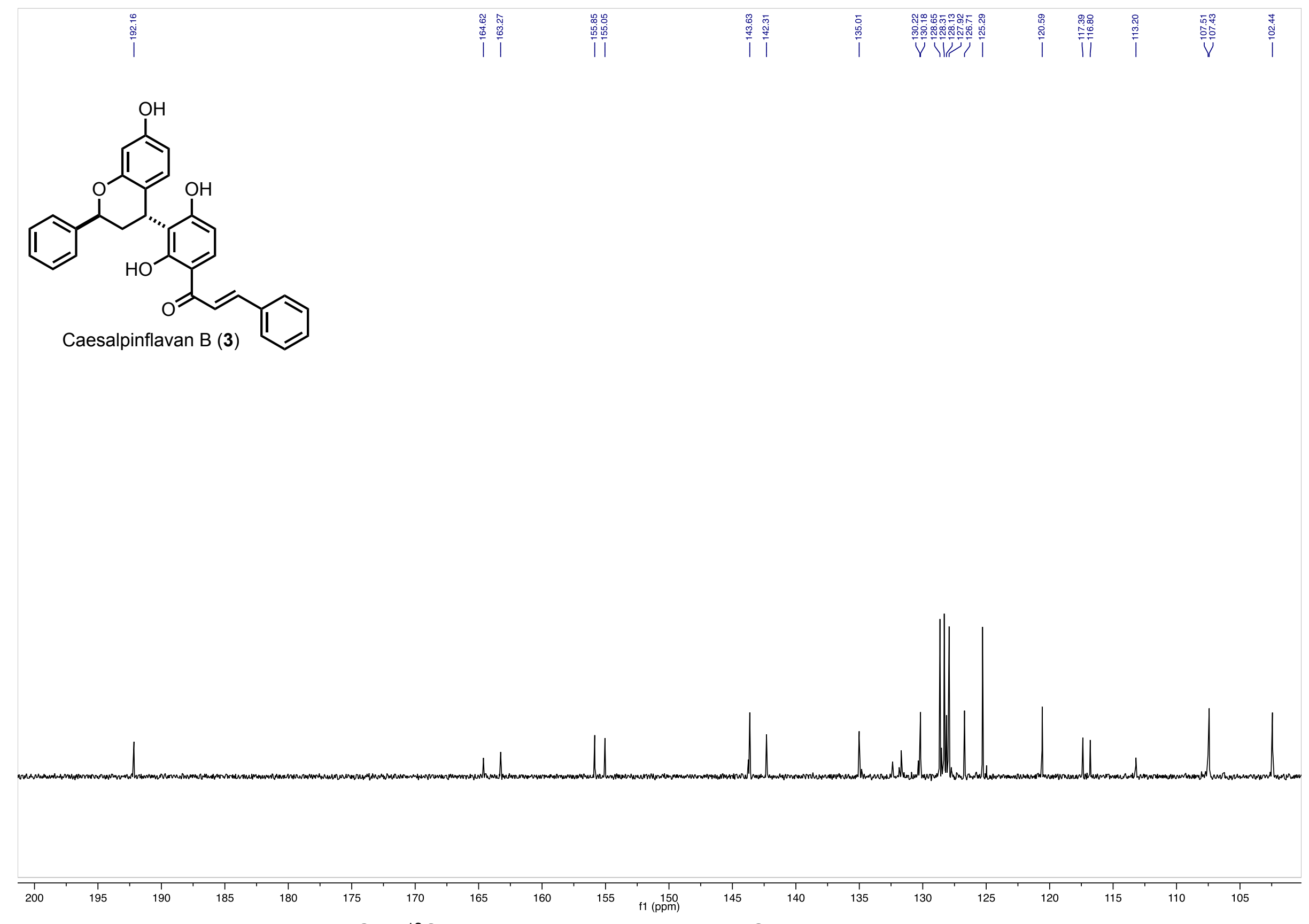

Figure S90. ${ }^{13} \mathrm{C}$ NMR (151 MHz, methanol- $\left.d_{4}\right)$, Caesalpinflavan B (3) (inset). 


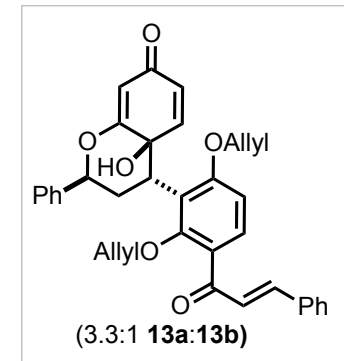

$\mathrm{CDCl}_{3}$
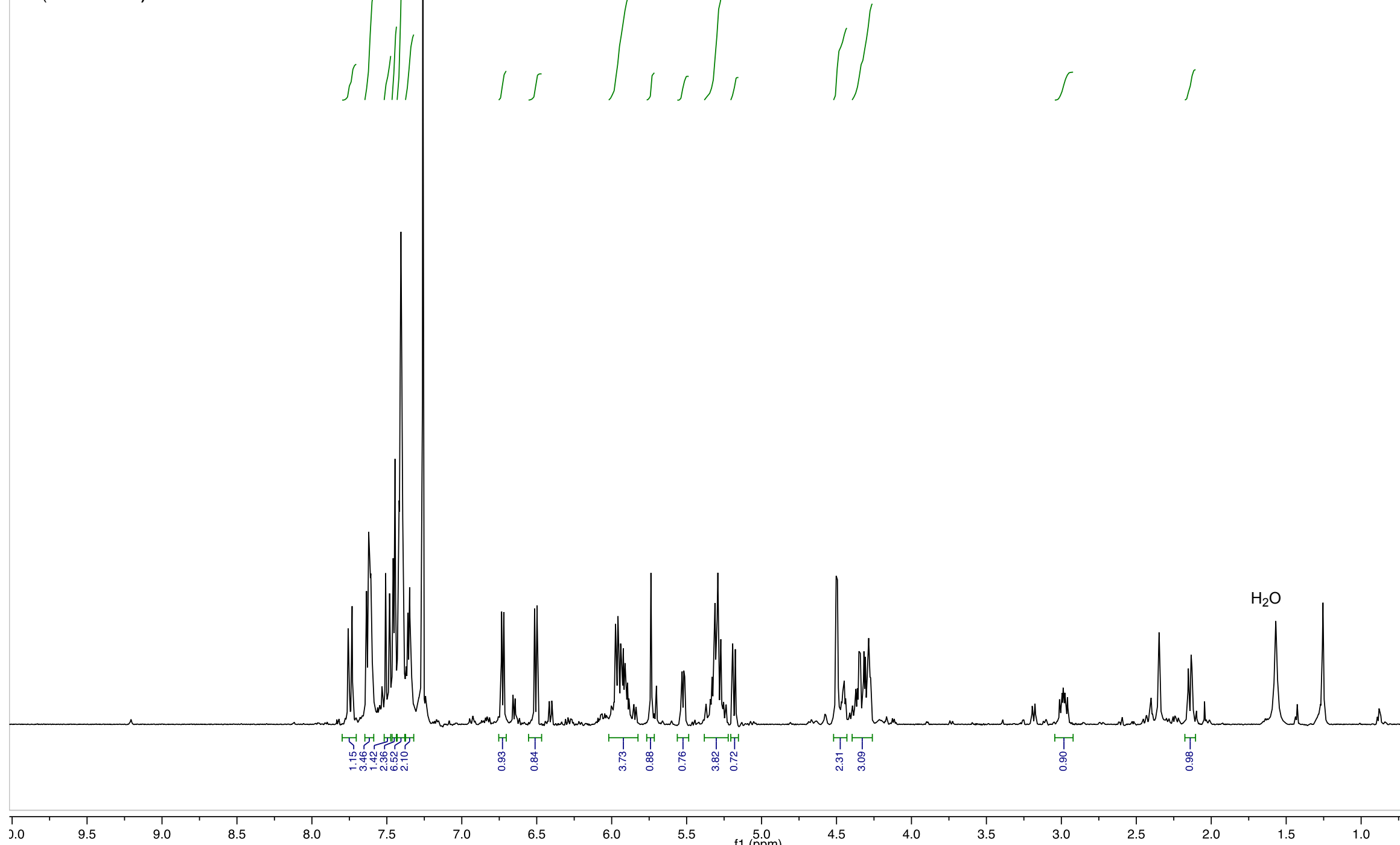

Figure S91. ${ }^{1} \mathrm{H}$ NMR (600 MHz, chloroform- $d$ ), 19a. 


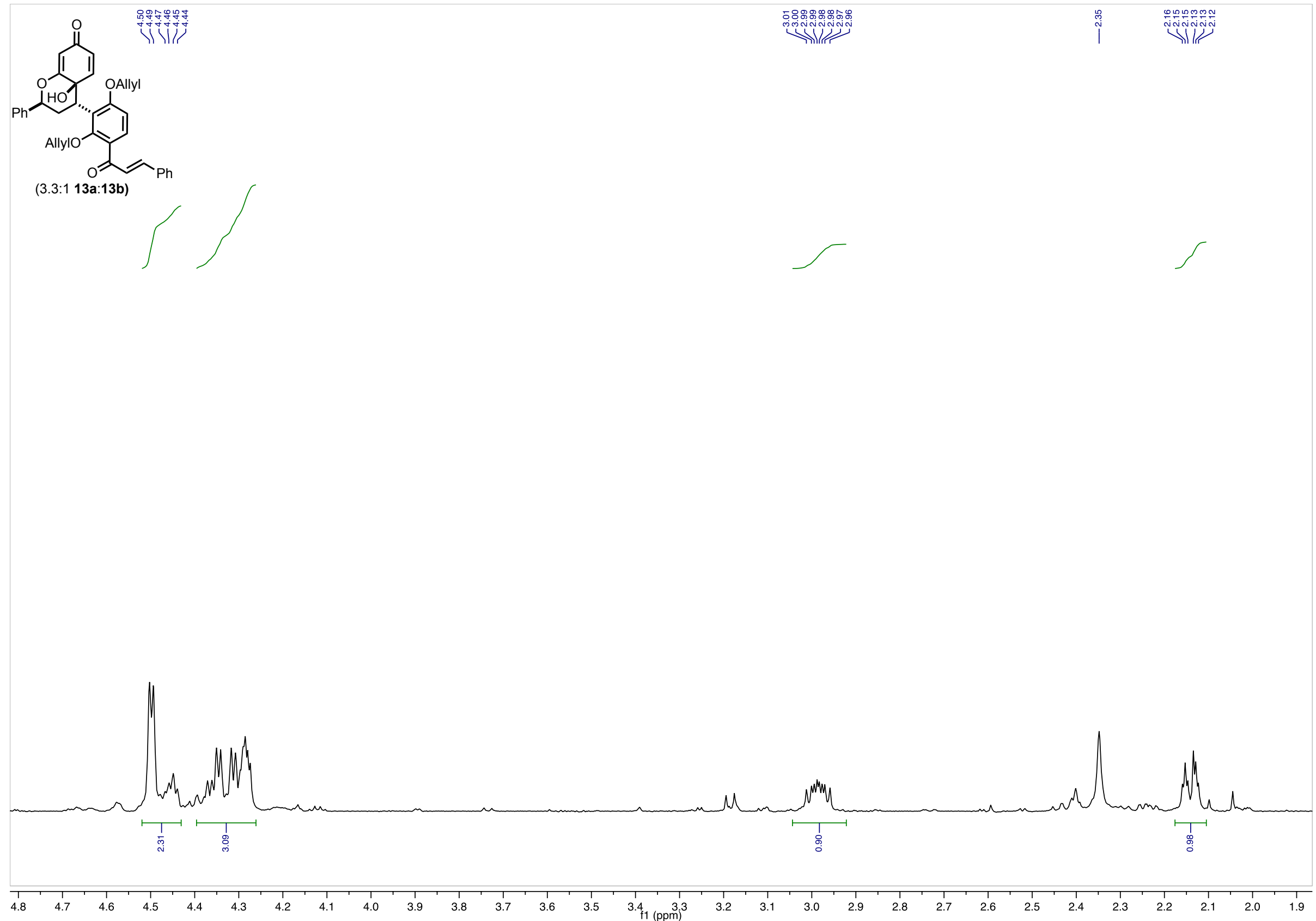

Figure S92. ${ }^{1 \mathrm{H}} \mathrm{NMR}(600 \mathrm{MHz}$, chloroform- $d$ ), 19a (inset). 


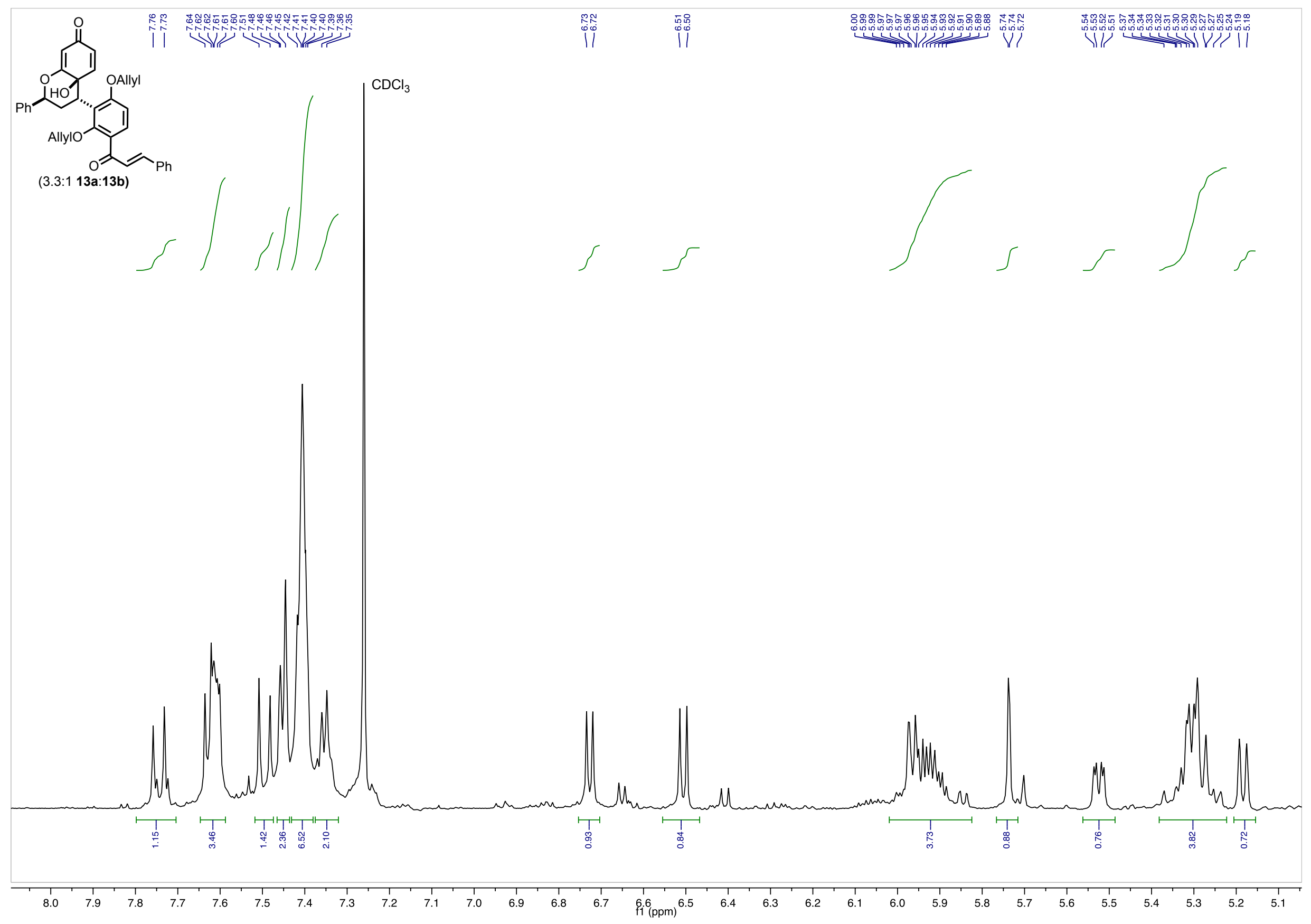

Figure S93. ${ }^{1} \mathrm{H}$ NMR (600 MHz, chloroform- $d$ ), 19a (inset). 


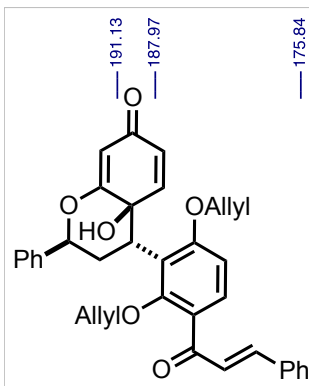

(3.3:1 13a:13b)
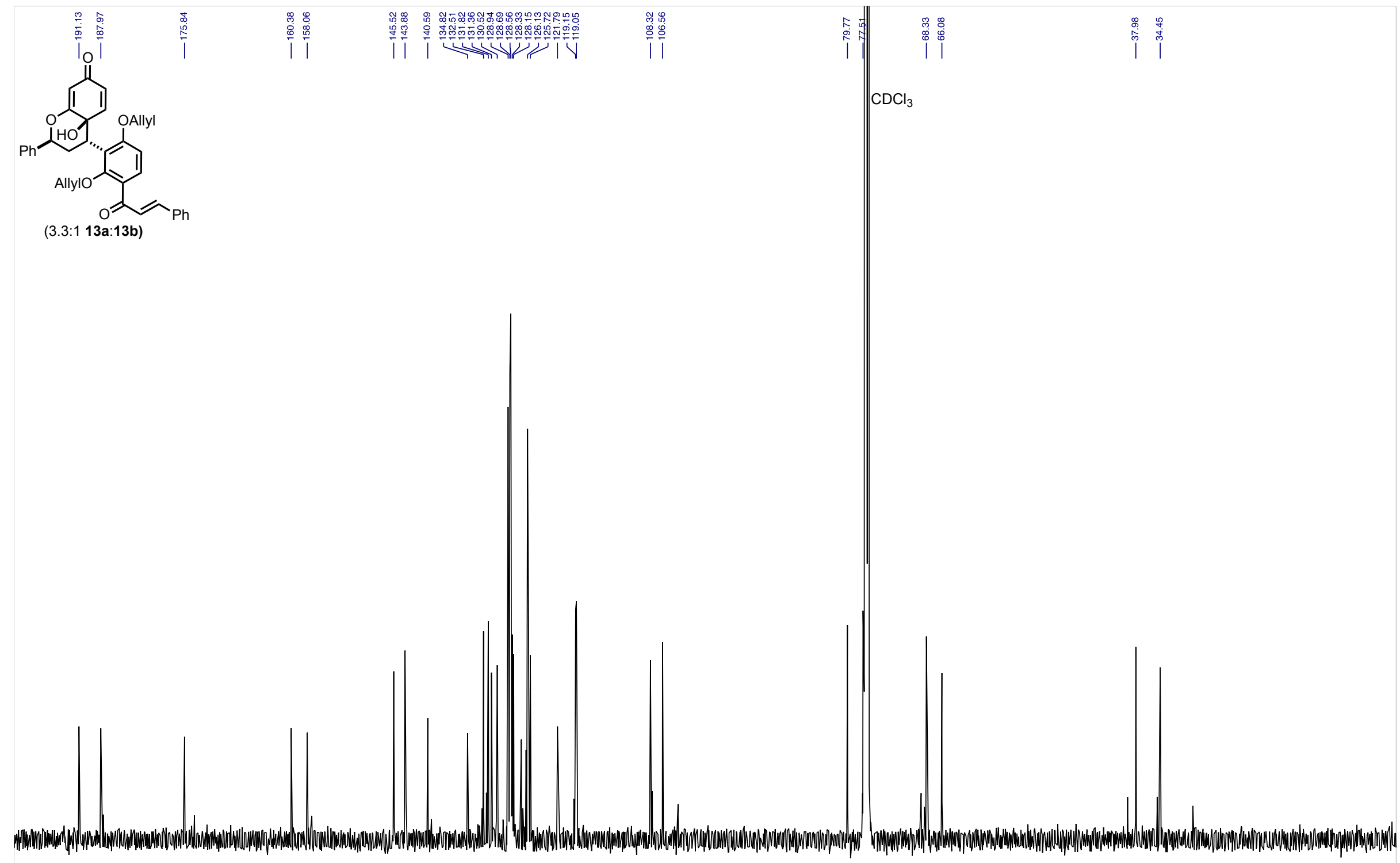

Figure S94. ${ }^{13} \mathrm{C}$ NMR (151 MHz, chloroform- $d$ ), 19a. 

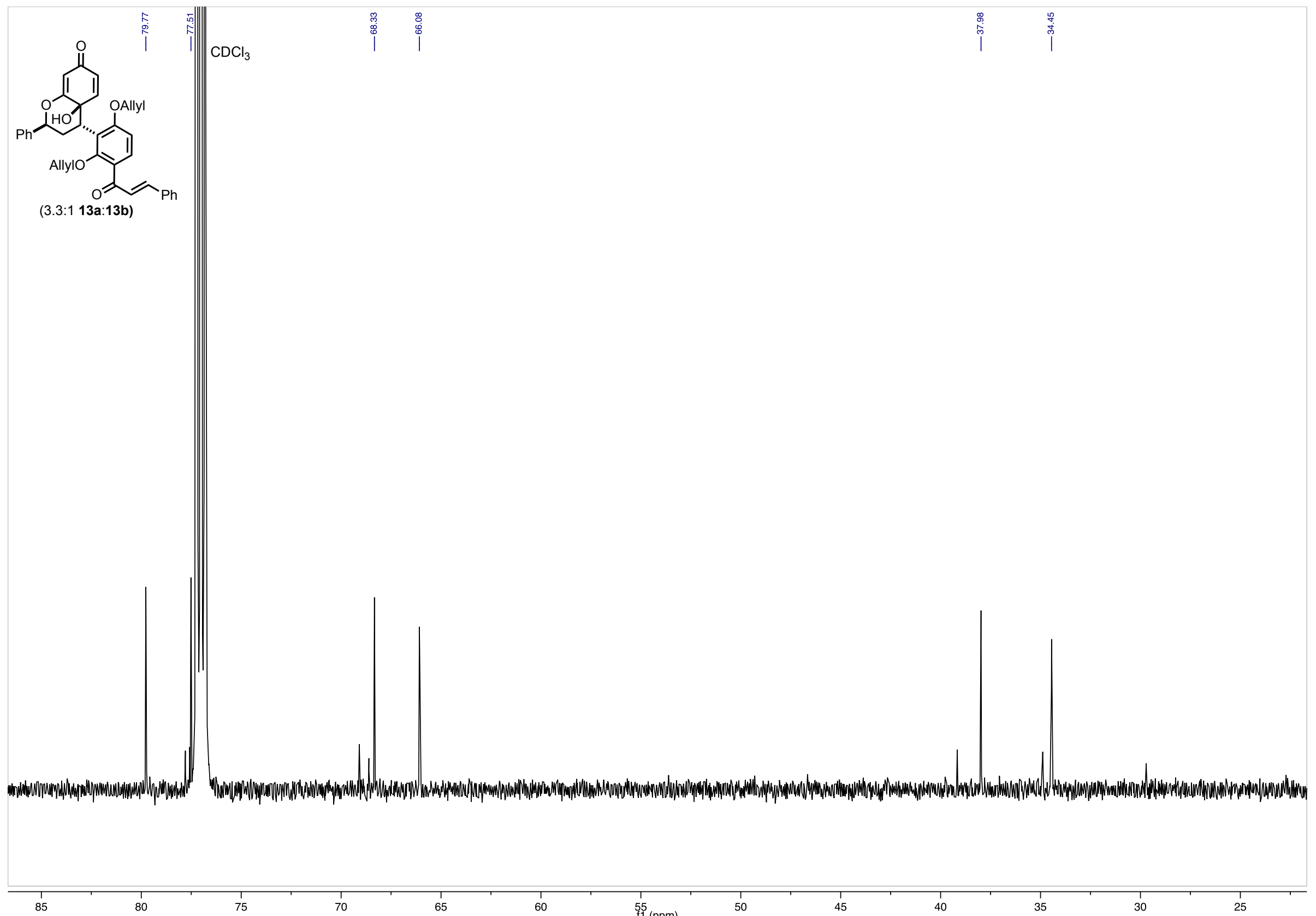

Figure S95. ${ }^{13} \mathrm{C}$ NMR (151 MHz, chloroform-d), 19a (inset). 


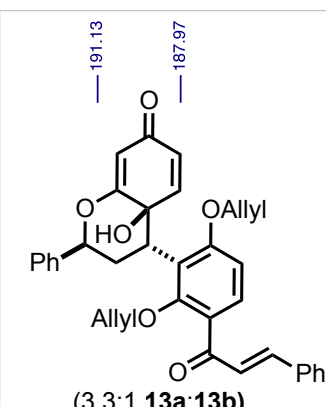

(3.3:1 13a:13b)

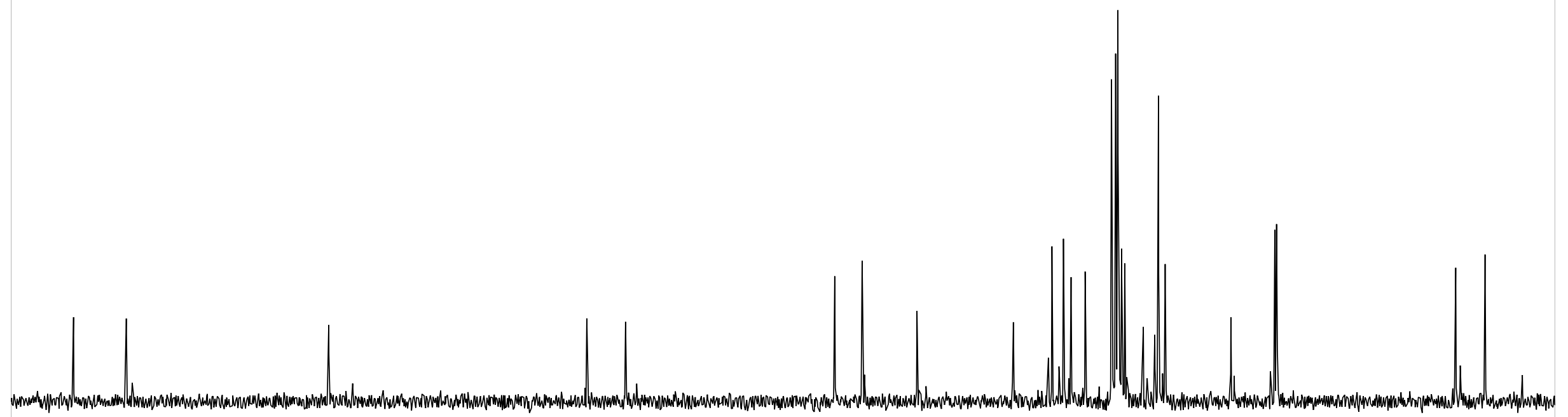

Figure S96. ${ }^{13} \mathrm{C}$ NMR (151 MHz, chloroform- $d$ ), 19a (inset). 


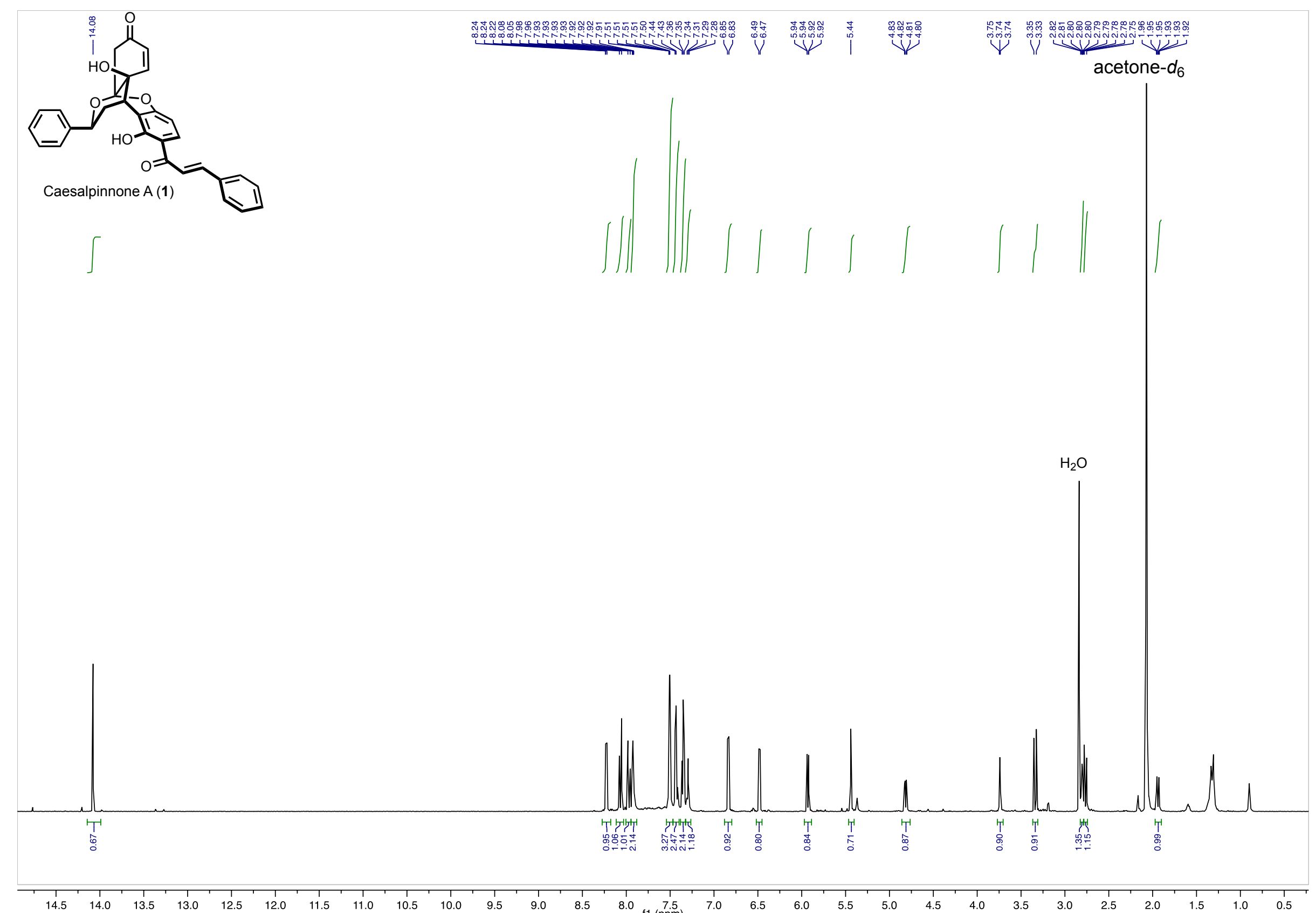

Figure S97. ${ }^{1} \mathrm{H}$ NMR (600 MHz, acetone- $\left.d_{6}\right)$, Caesalpinnone A (1). 


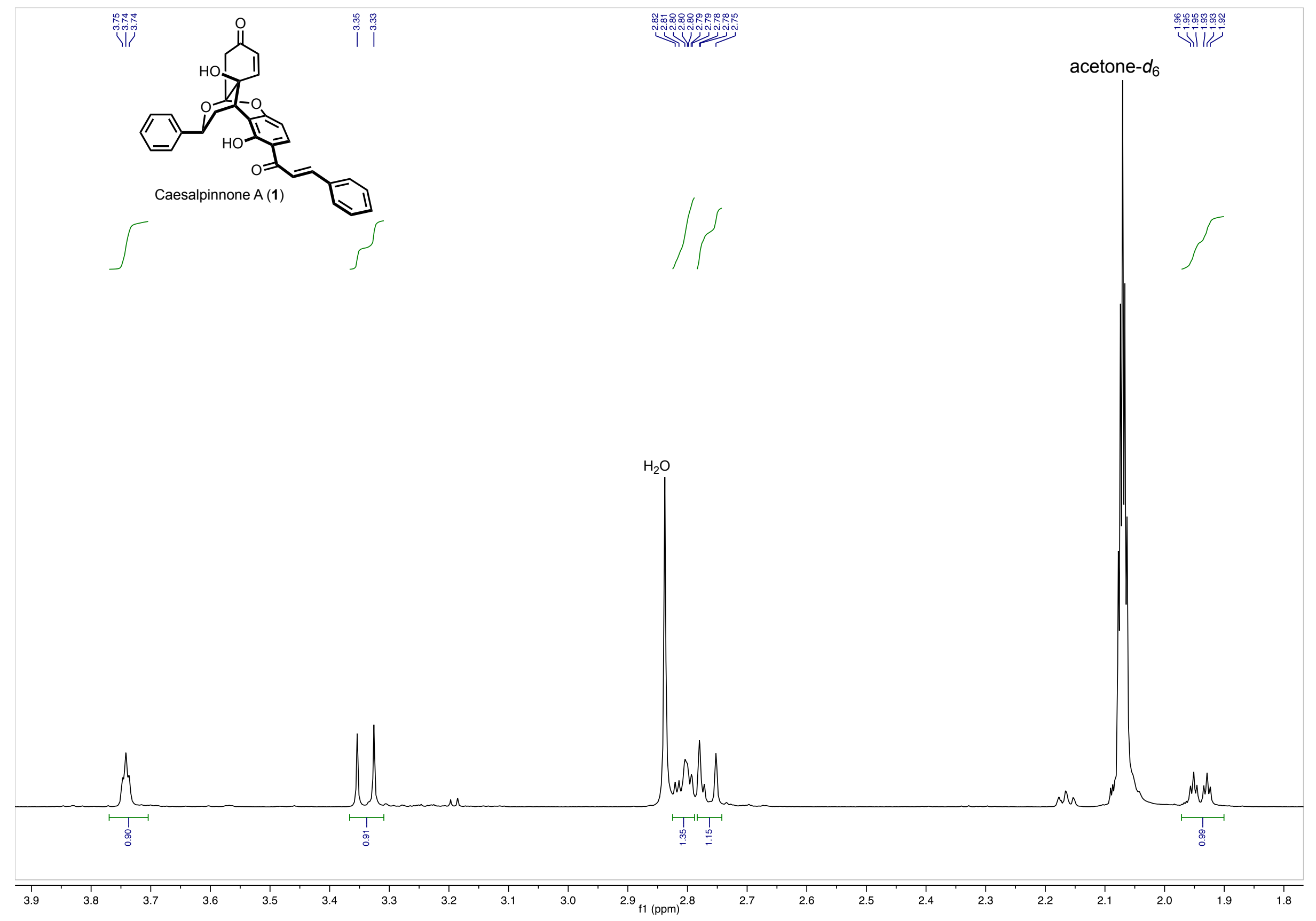

Figure S98. ${ }^{1} \mathrm{H}$ NMR $\left(600 \mathrm{MHz}\right.$, acetone- $\left.d_{6}\right)$, Caesalpinnone A (1) (inset). 

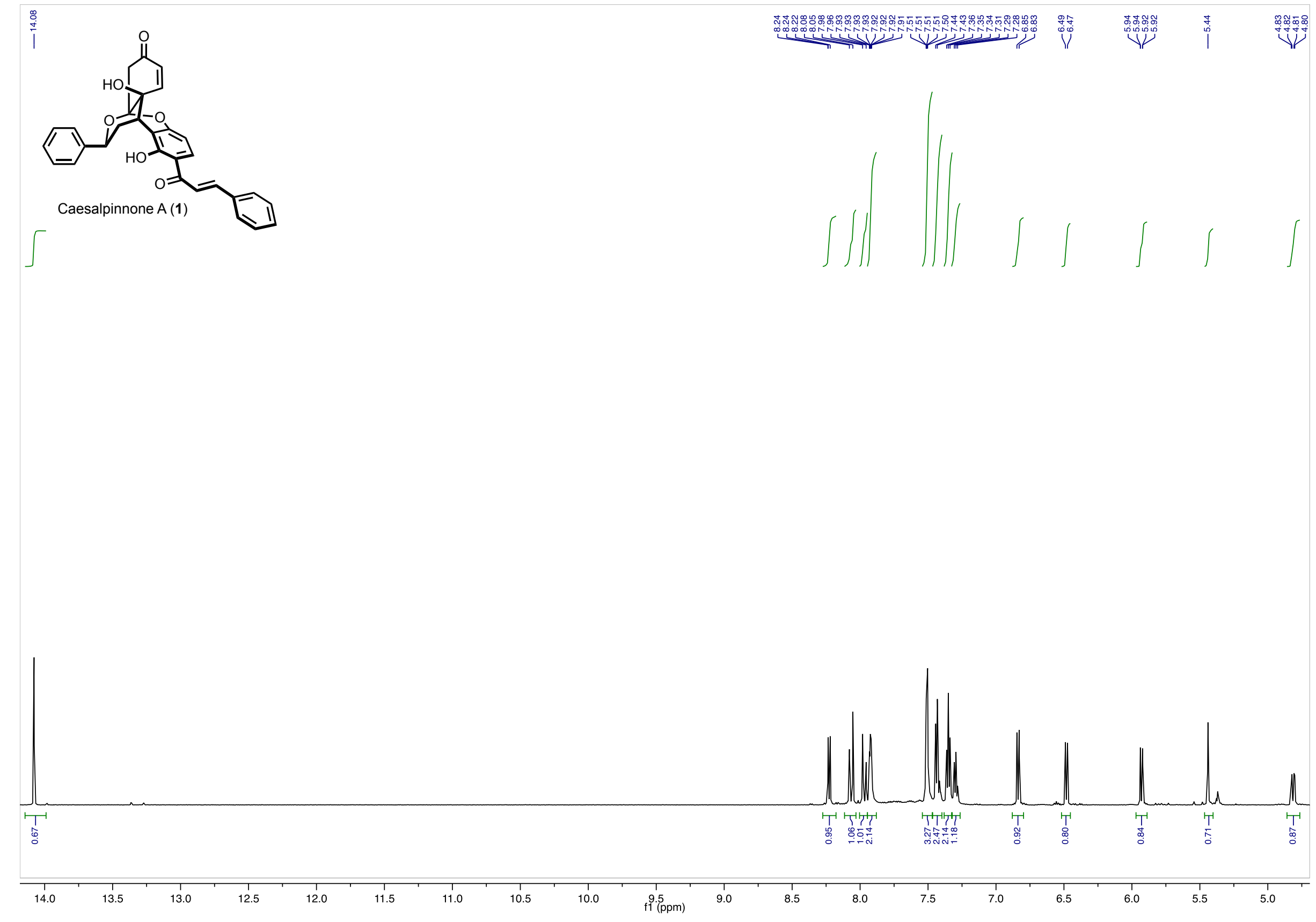

Figure S99. ${ }^{1} \mathrm{H}$ NMR (600 MHz, acetone-d6), Caesalpinnone A (1) (inset). 


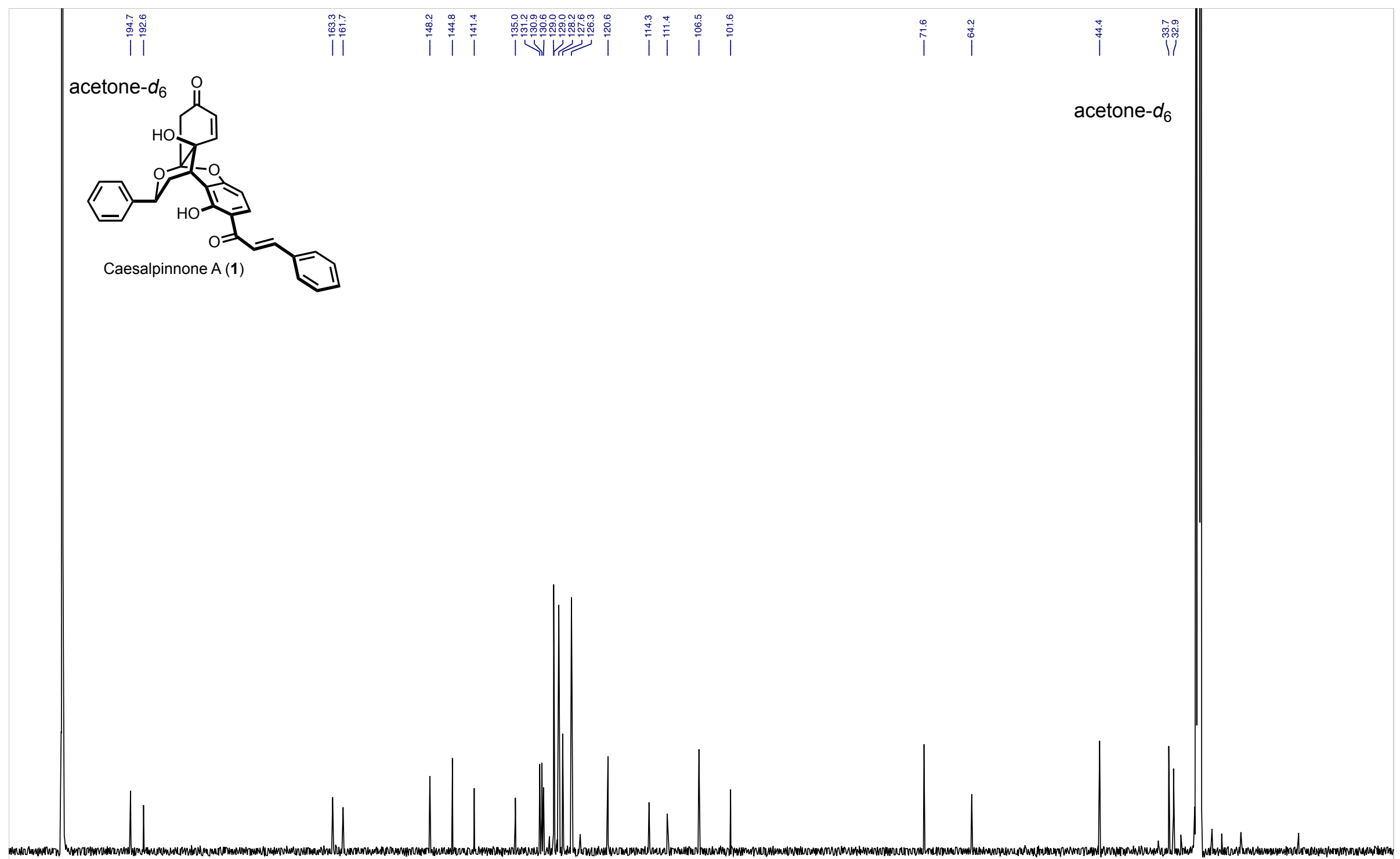

210

$200 \quad 190$

180

Figure S100. ${ }^{13} \mathrm{C}$ NMR (151 MHz, acetone- $\left.d_{6}\right)$, Caesalpinnone A (1). 


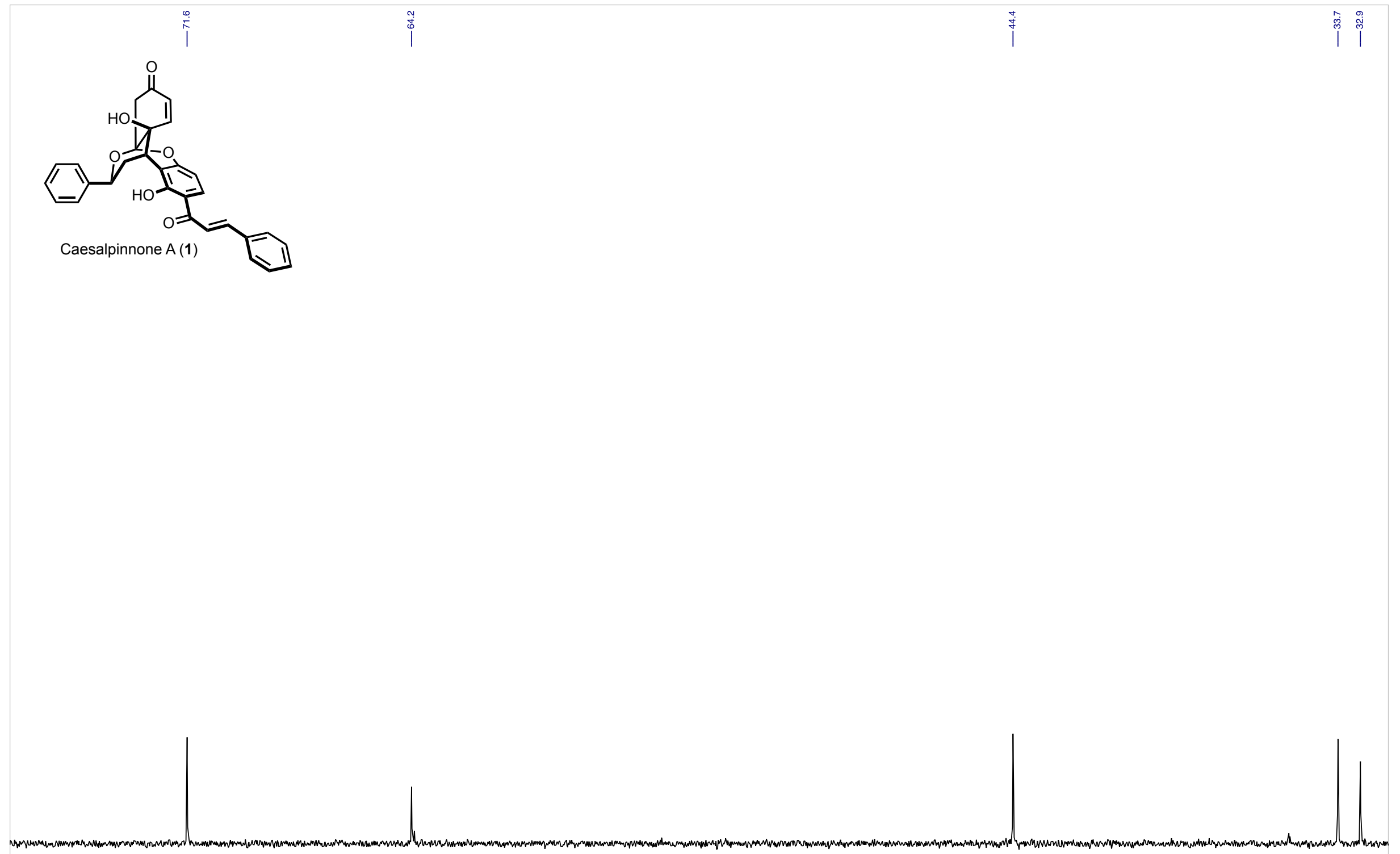

75

70

65

60

55
$\mathrm{f1}(\mathrm{ppm})$

50

45

40

Figure S101. ${ }^{13} \mathrm{C}$ NMR (151 MHz, acetone-d6), Caesalpinnone A (1) (inset). 


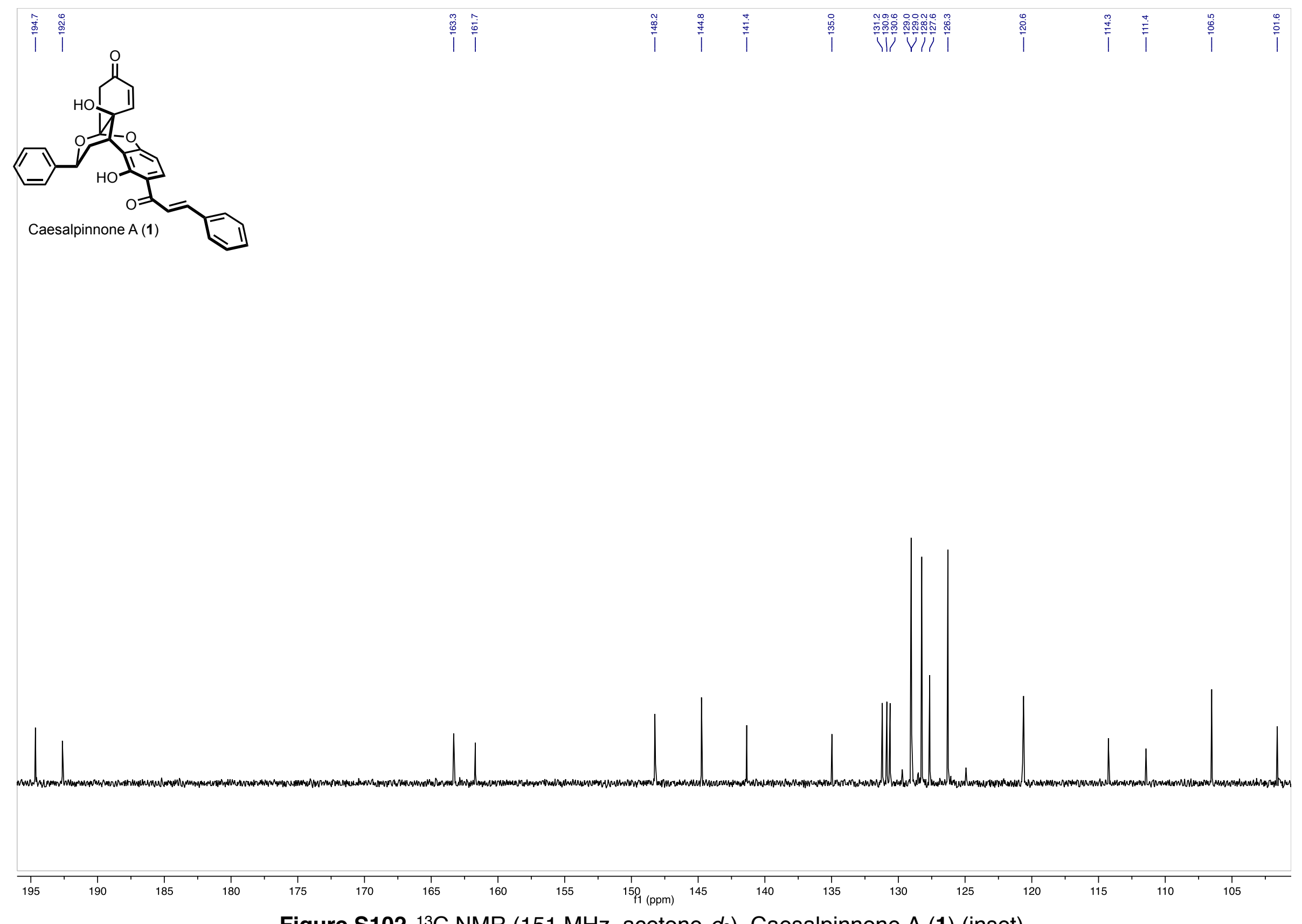

Figure S102. ${ }^{13} \mathrm{C}$ NMR (151 MHz, acetone- $d 6$ ), Caesalpinnone A (1) (inset). 


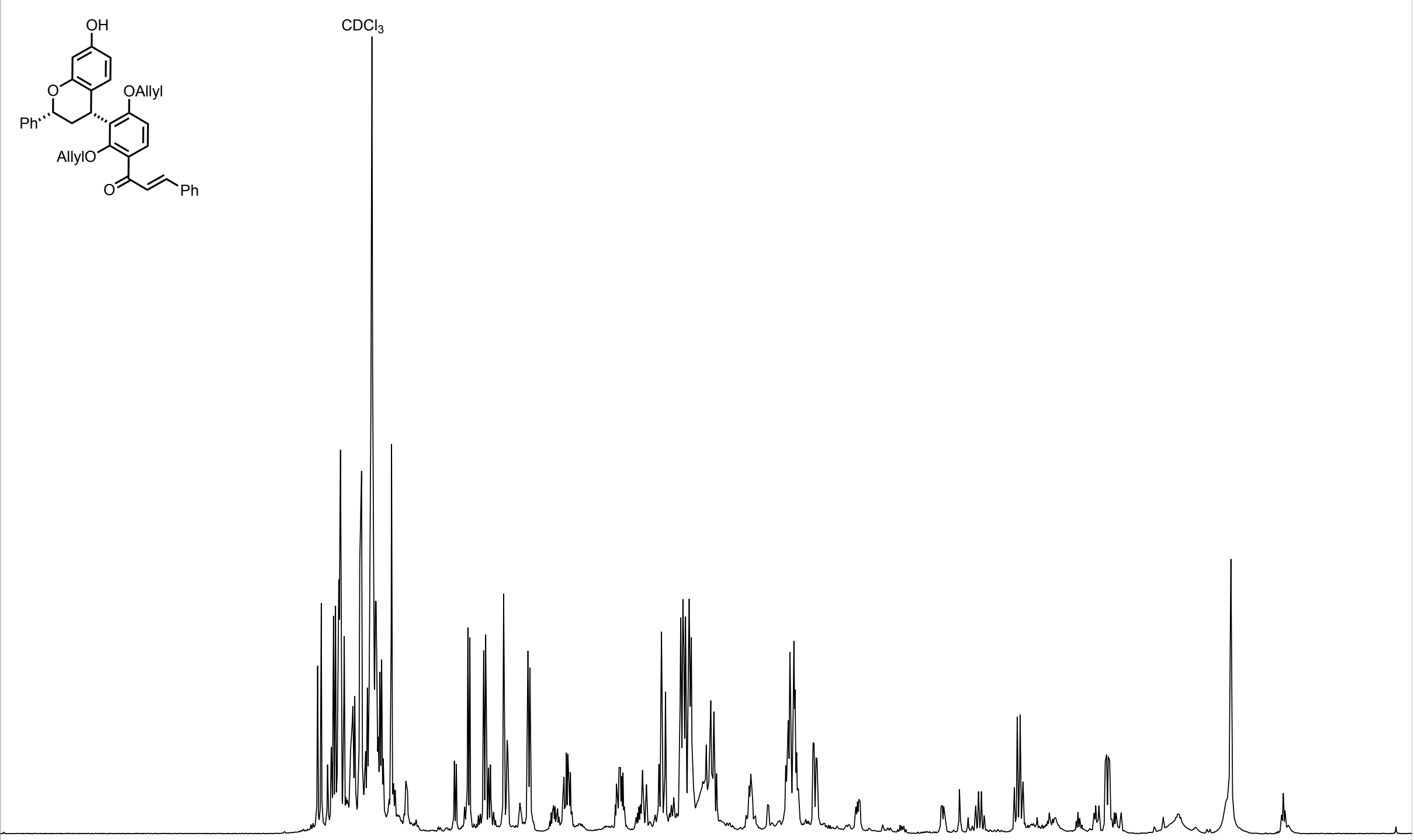

Figure S103. ${ }^{1 H} \mathrm{NMR}(600 \mathrm{MHz}$, chloroform- $d$ ), 18b. 

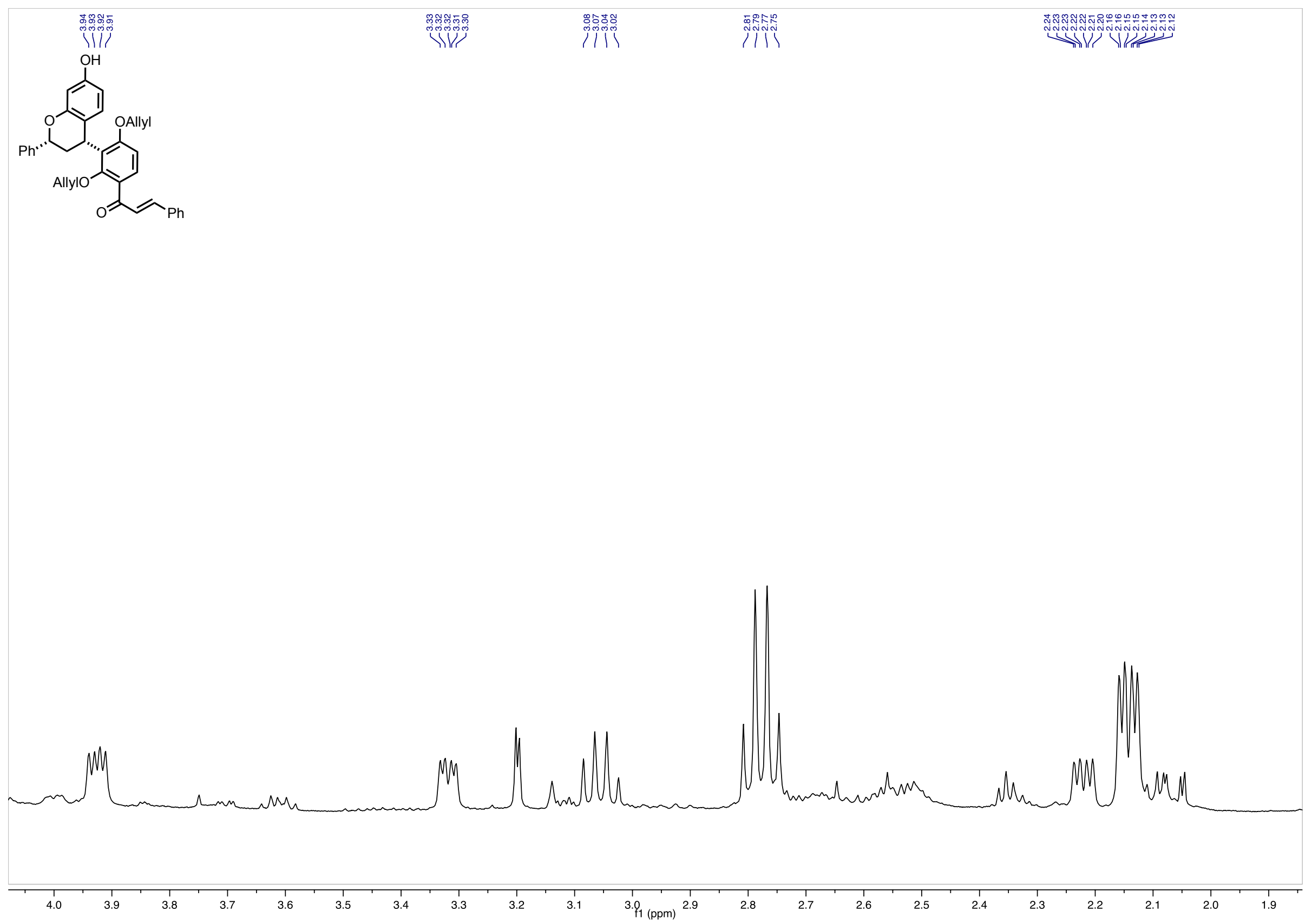

Figure S104. ${ }^{1} \mathrm{H}$ NMR (600 MHz, chloroform- $d$ ), 18b (inset). 

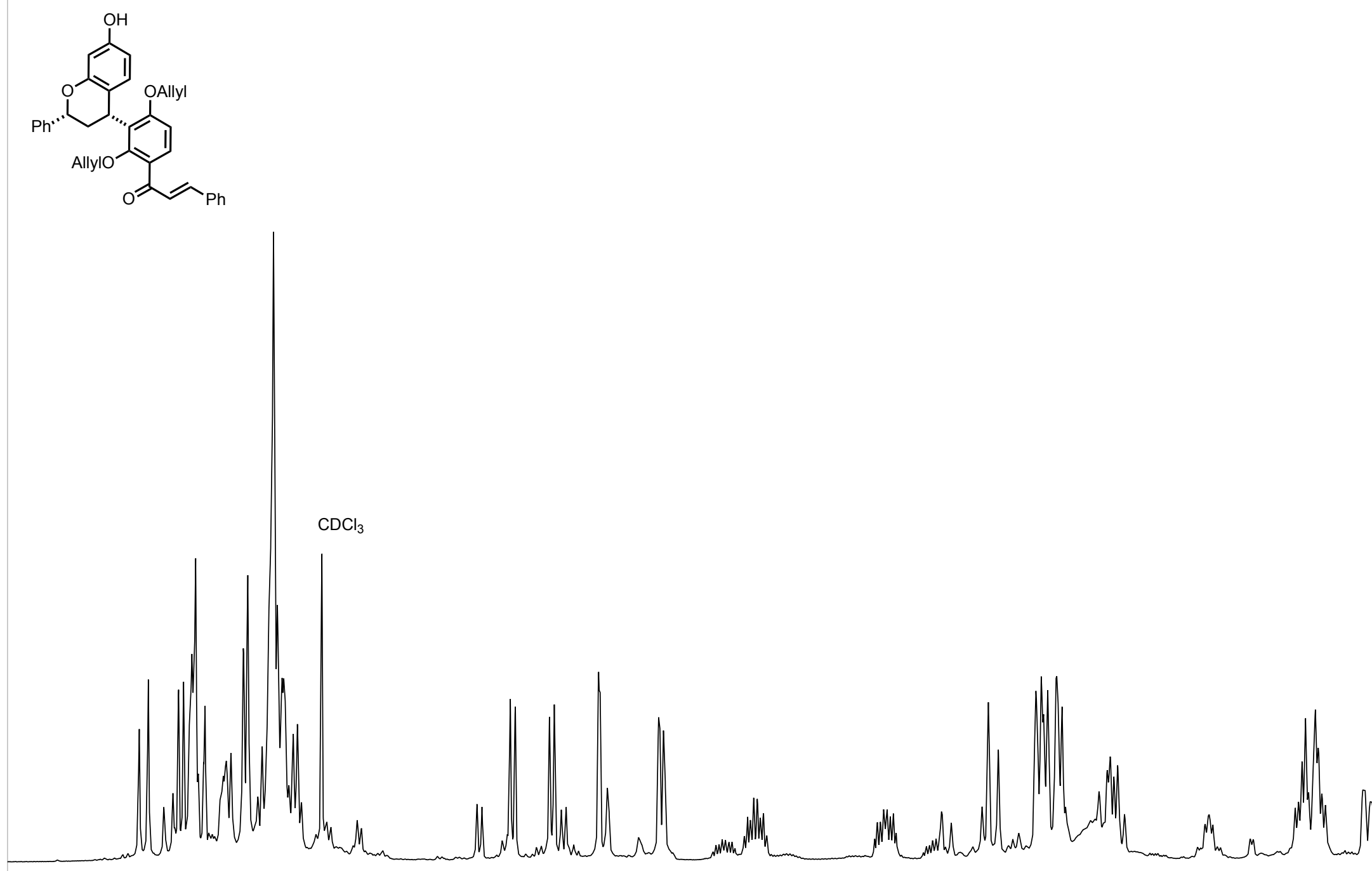

Figure S105. ${ }^{1} \mathrm{H}$ NMR (600 MHz, chloroform- $d$ ), 18b (inset). 

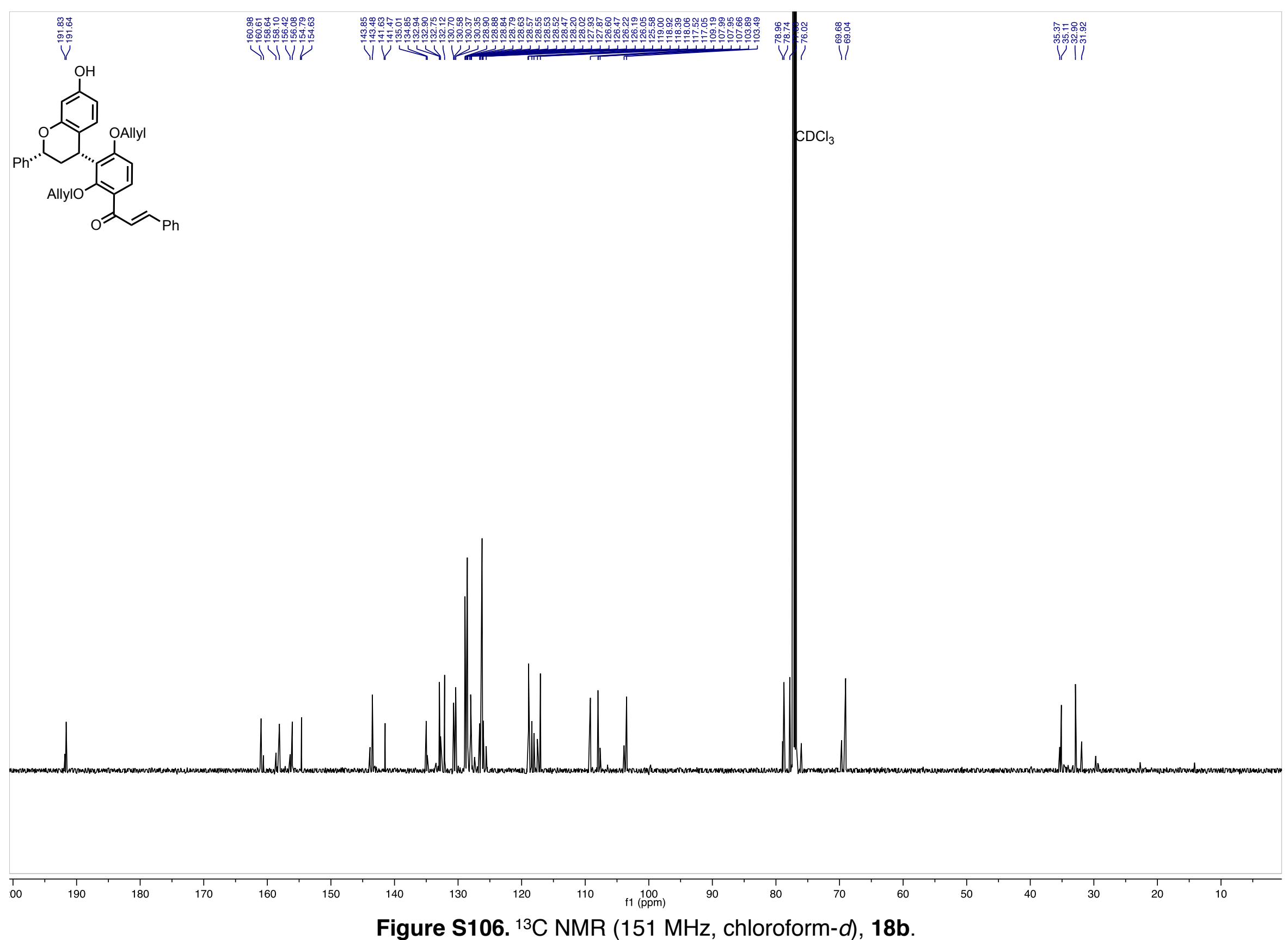

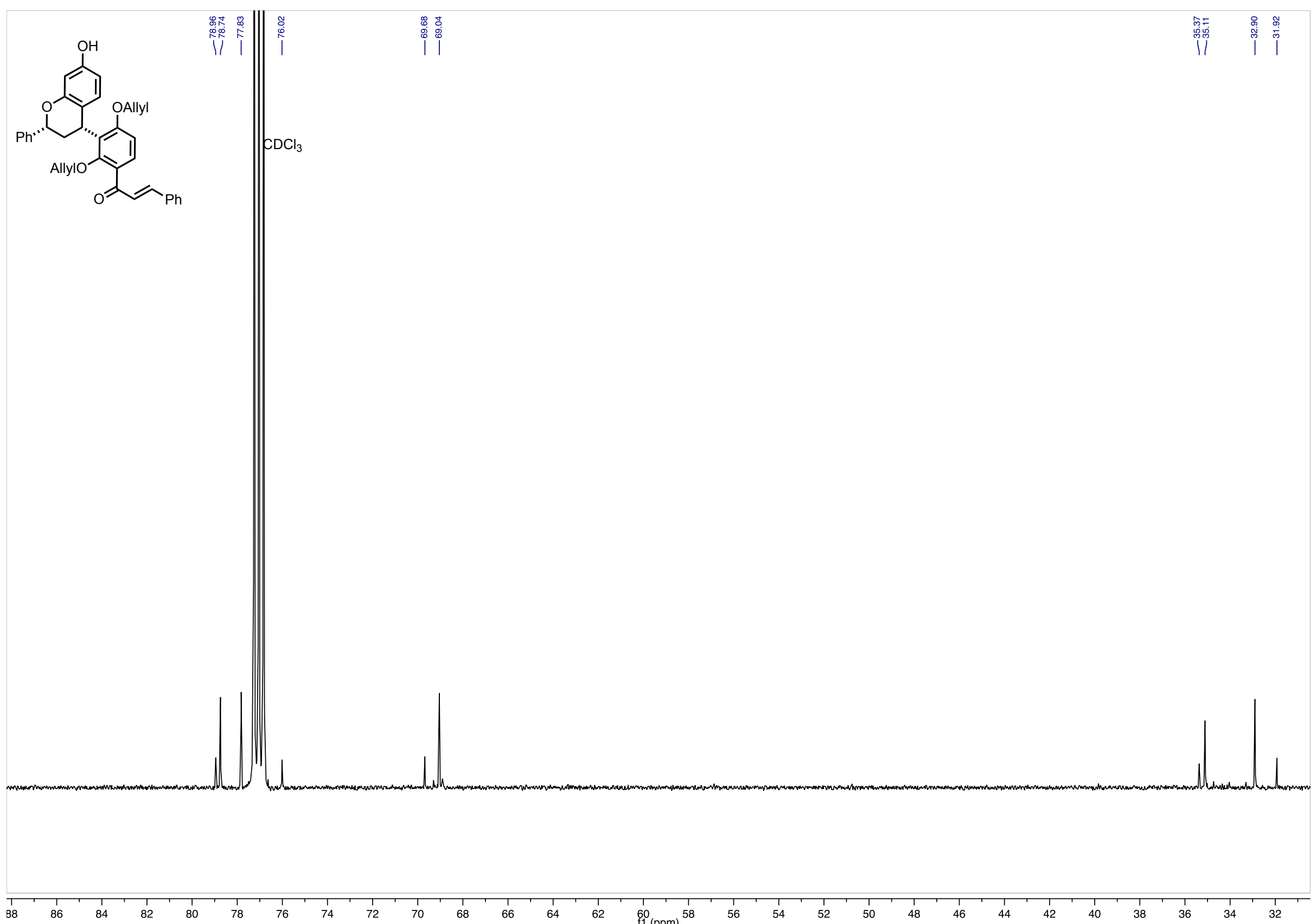

Figure S107. ${ }^{13} \mathrm{C}$ NMR (151 MHz, chloroform- $d$ ), 18b (inset). 


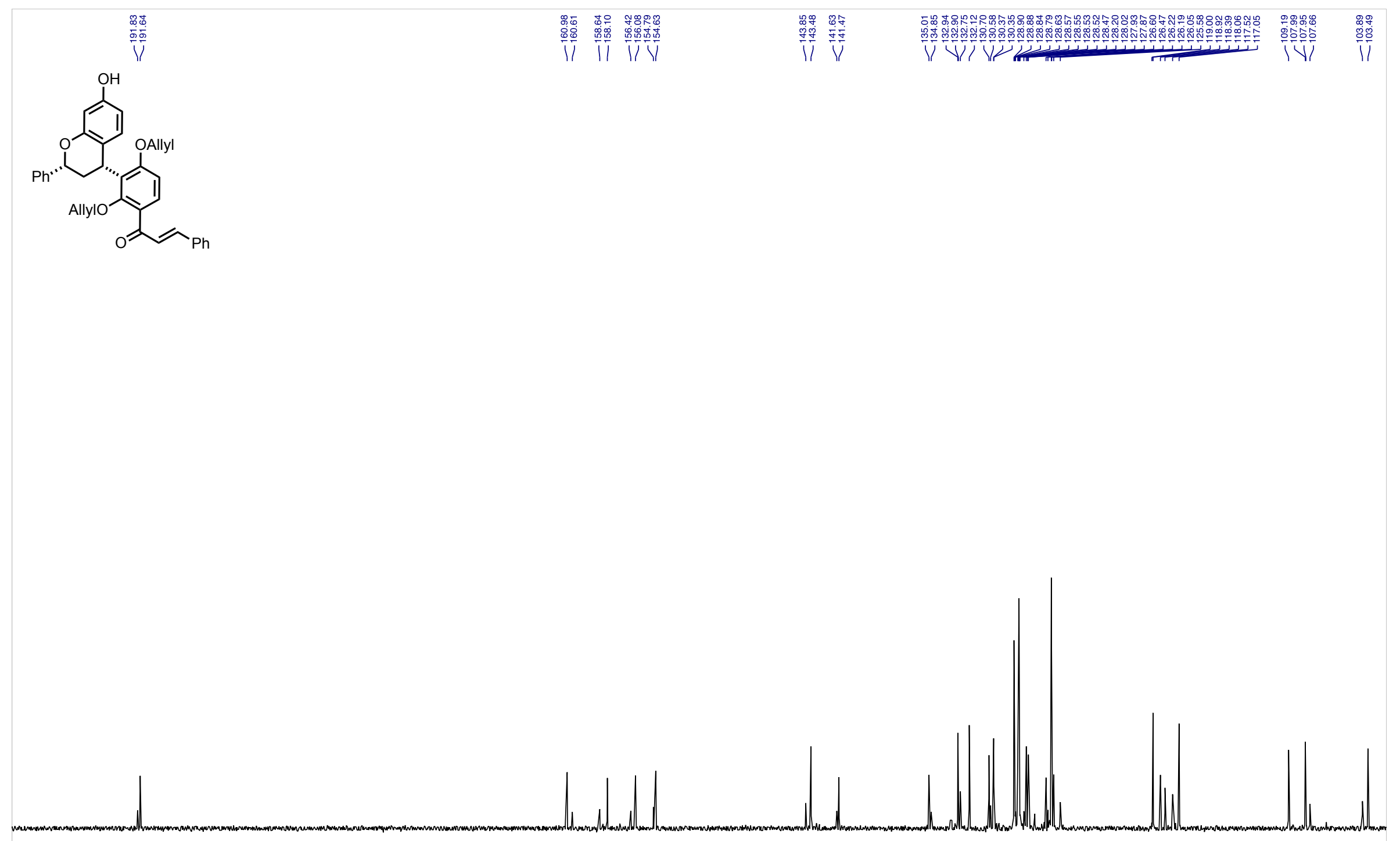

Figure S108. ${ }^{13} \mathrm{C}$ NMR (151 MHz, chloroform- $d$ ), 18b (inset). 


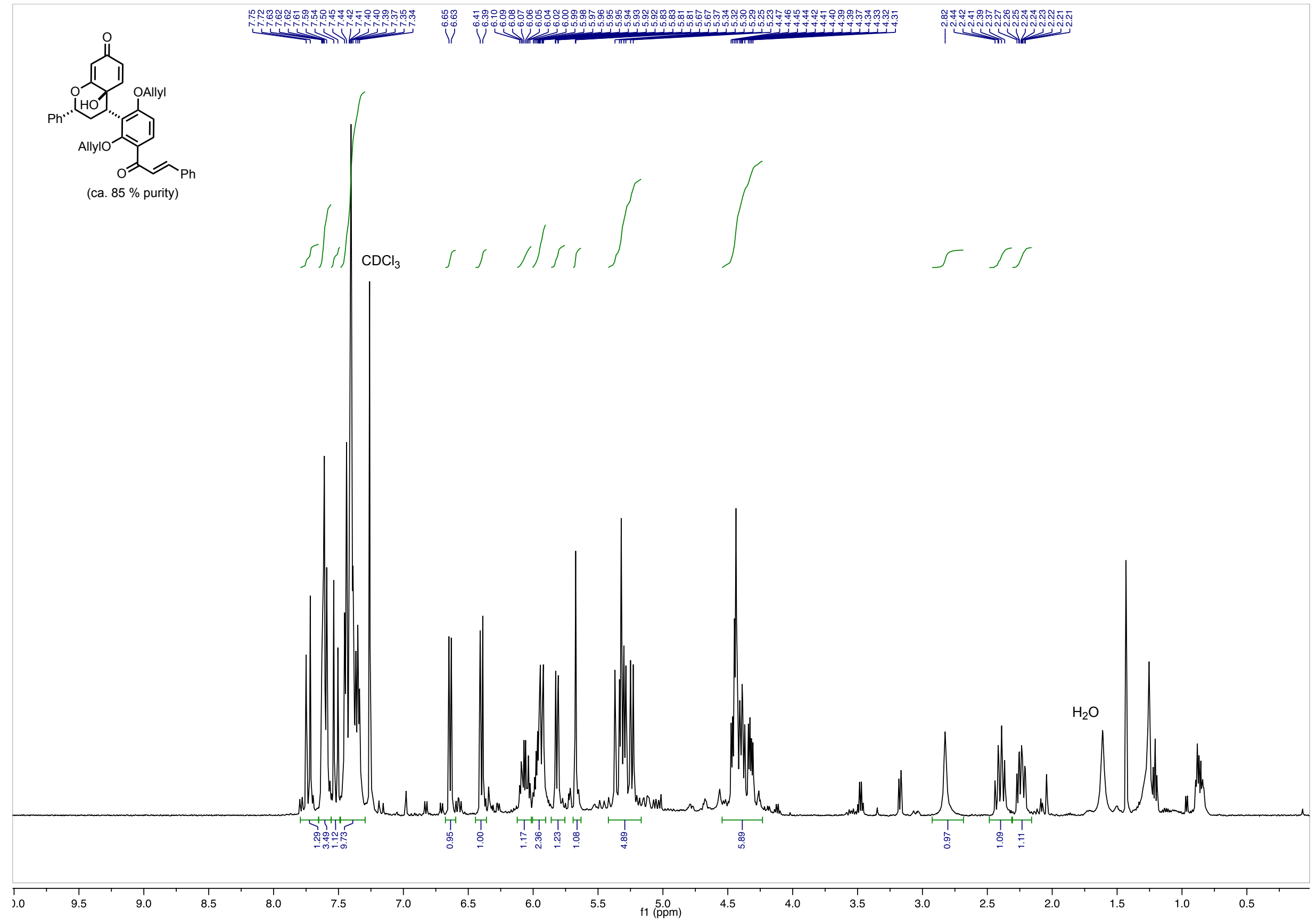

Figure S109. ${ }^{1} \mathrm{H}$ NMR (600 MHz, chloroform- $d$ ), $19 b$. 


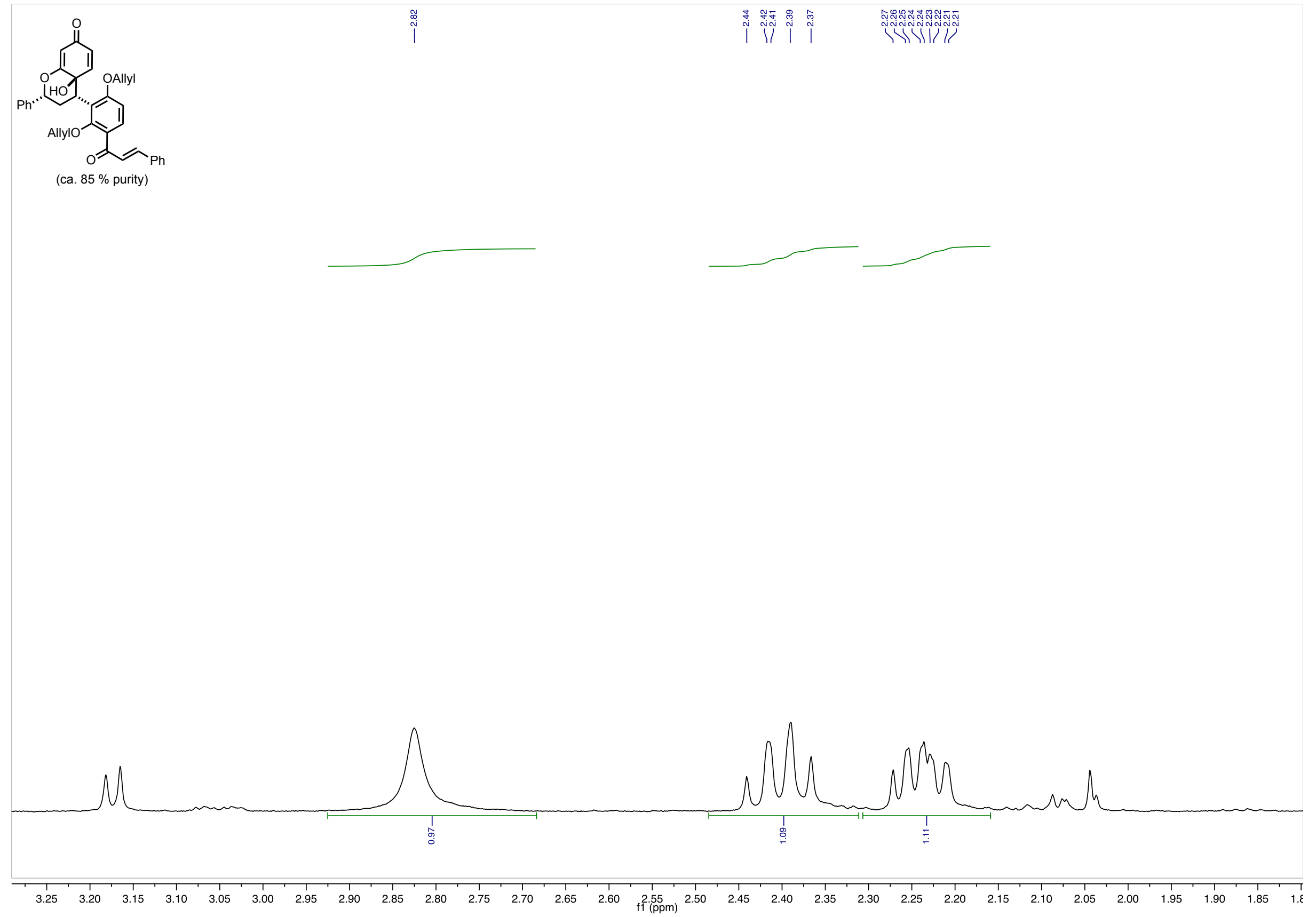

Figure S110. ${ }^{1} \mathrm{H}$ NMR (600 MHz, chloroform-d), 19b (inset). 


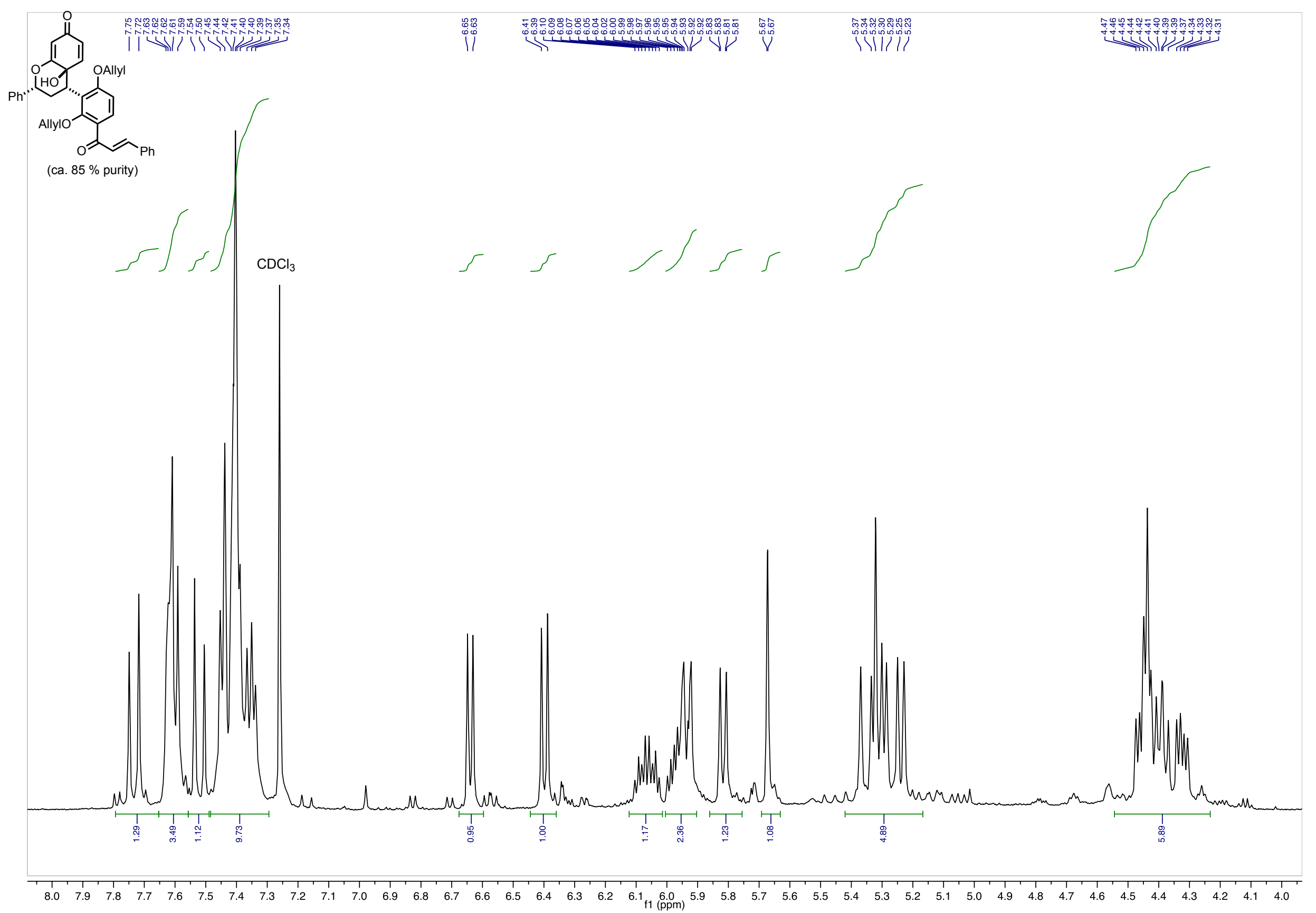

Figure S111. ${ }^{1} \mathrm{H}$ NMR (600 MHz, chloroform- $d$ ), 19b (inset). 

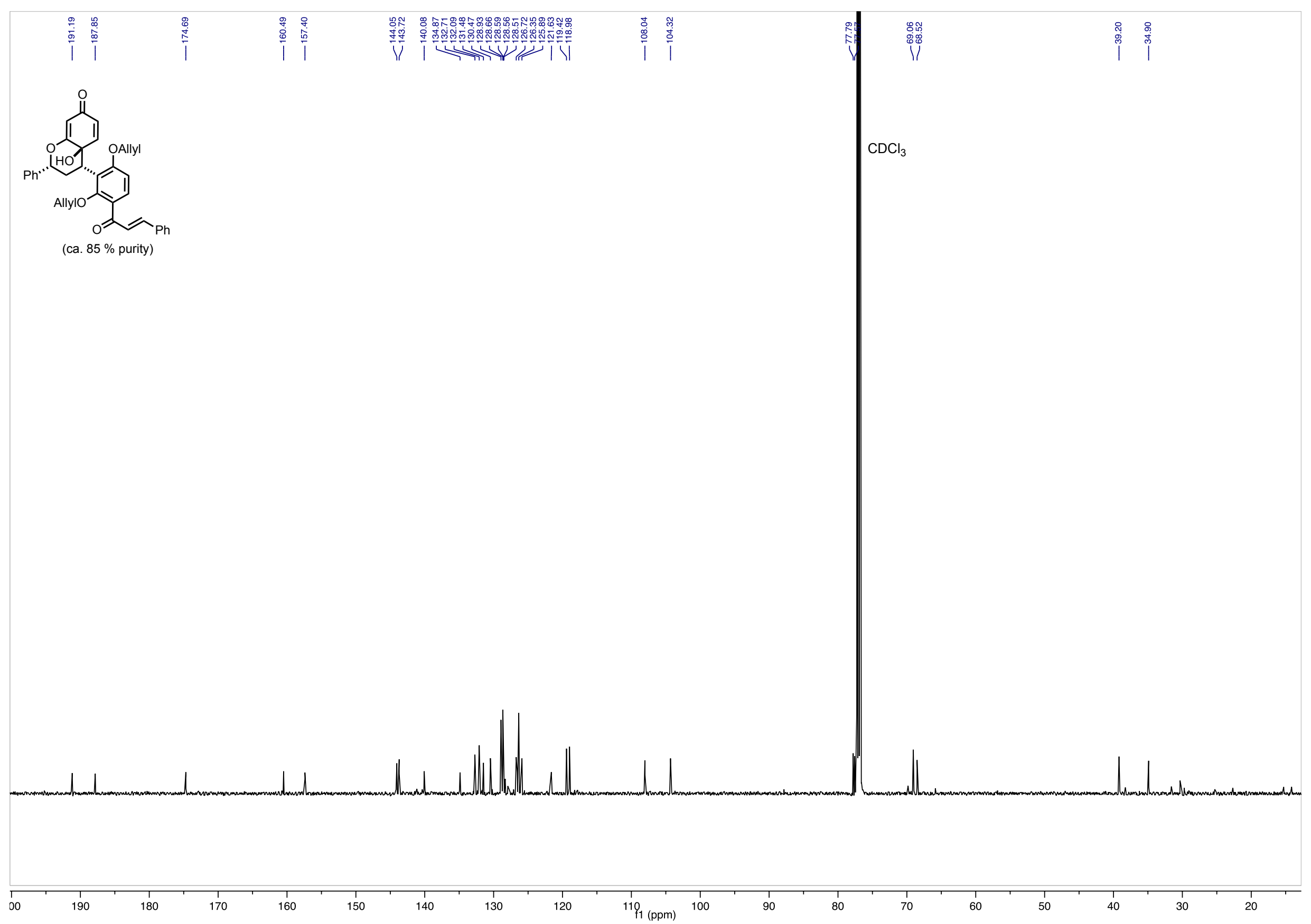

Figure S112. ${ }^{13} \mathrm{C}$ NMR (151 MHz, chloroform- $d$ ), $19 b$. 


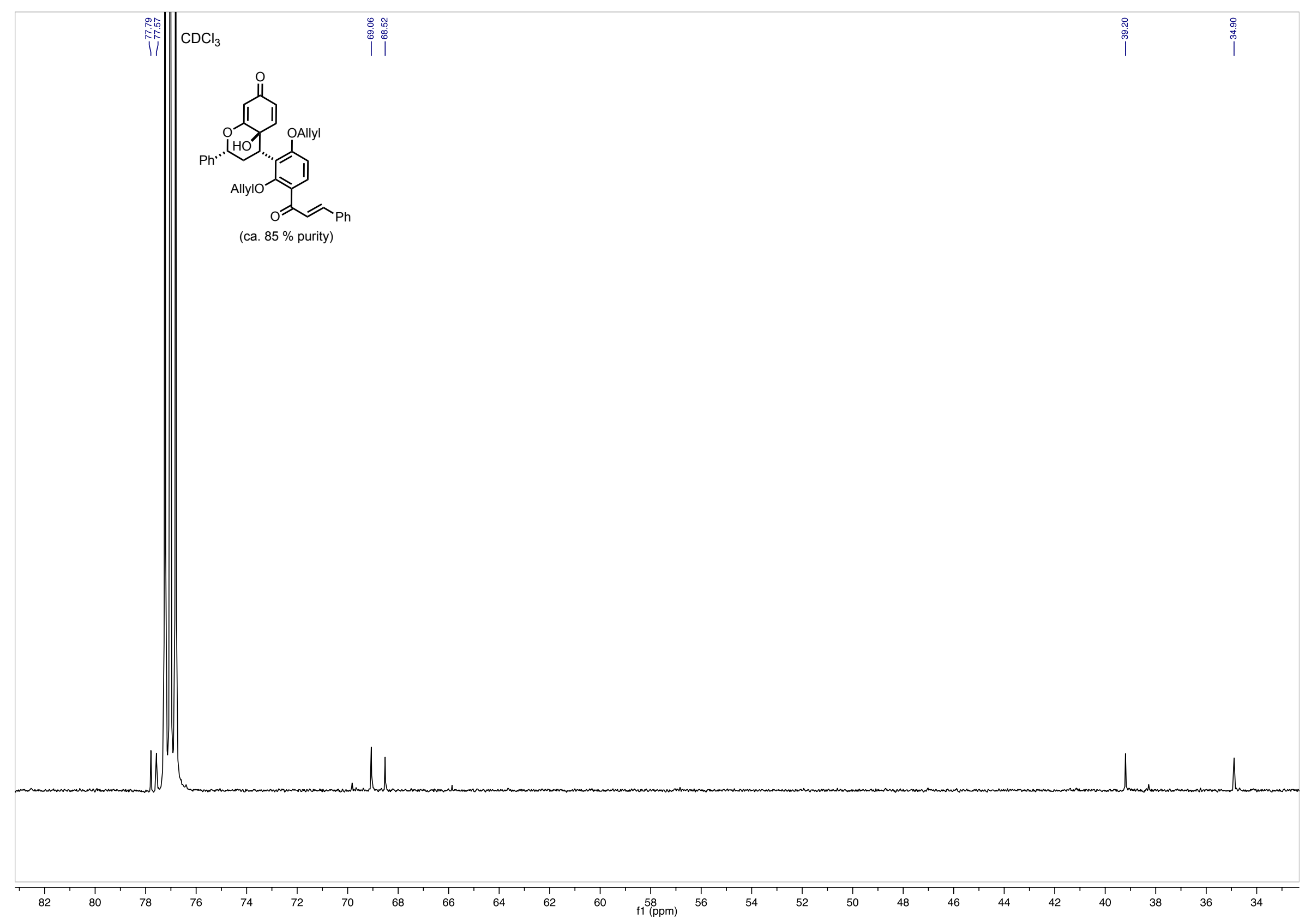

Figure S113. ${ }^{13} \mathrm{C}$ NMR (151 MHz, chloroform-d), 19b (inset). 

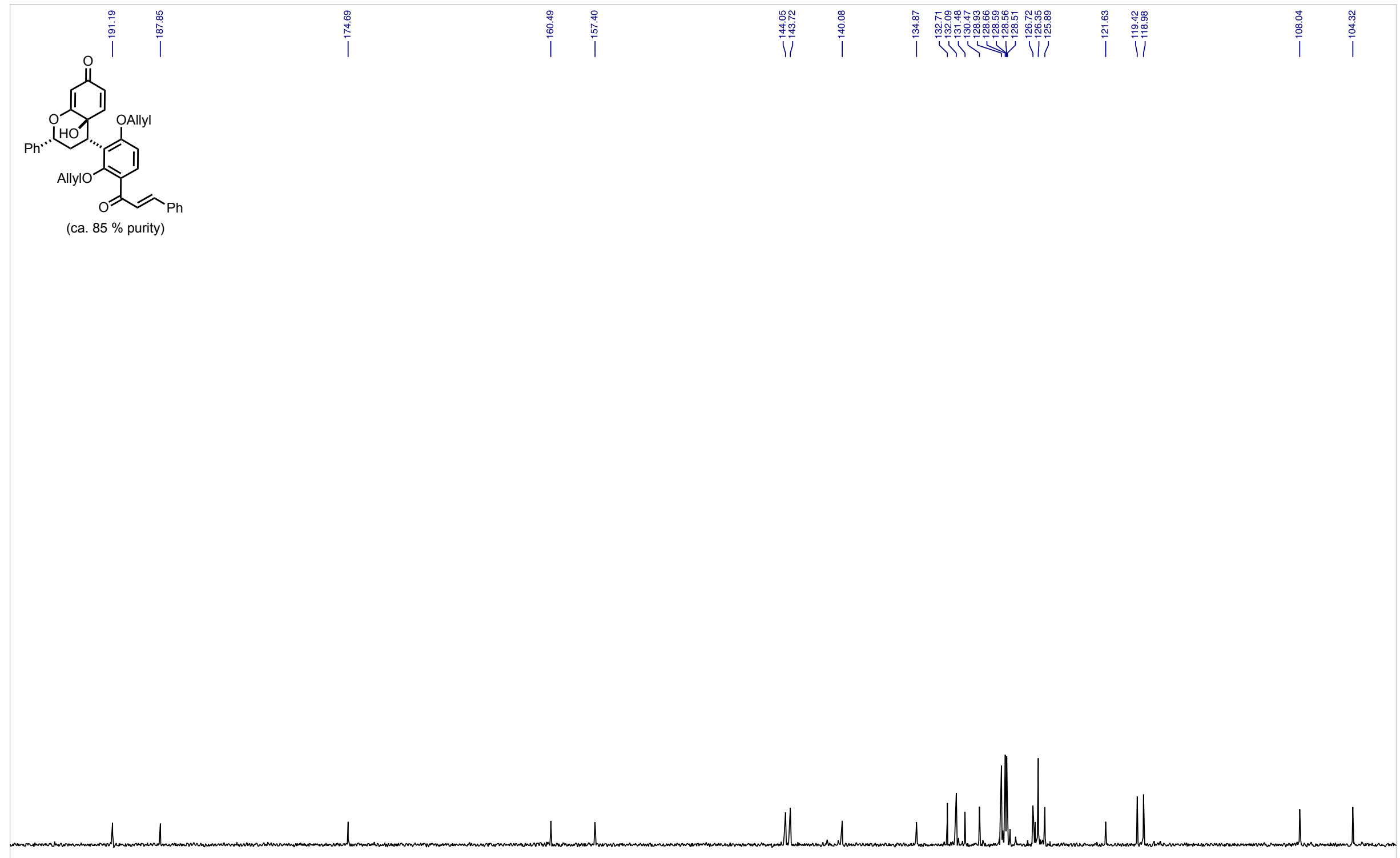

Figure S114. ${ }^{13} \mathrm{C}$ NMR (151 MHz, chloroform- $d$ ), 19b (inset). 


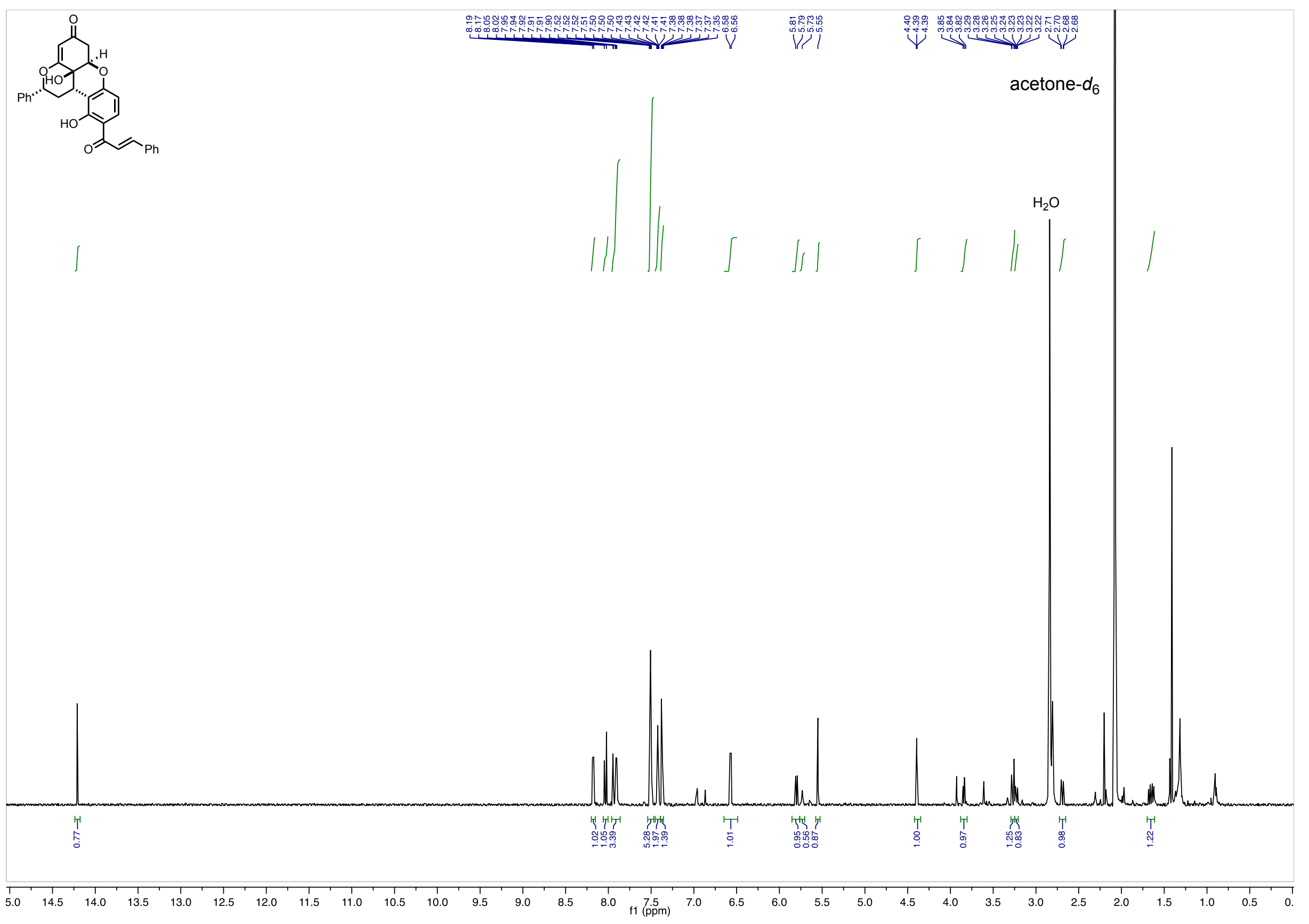

Figure S115. ${ }^{1} \mathrm{H}$ NMR (600 MHz, acetone- $\left.d_{6}\right), 21$. 


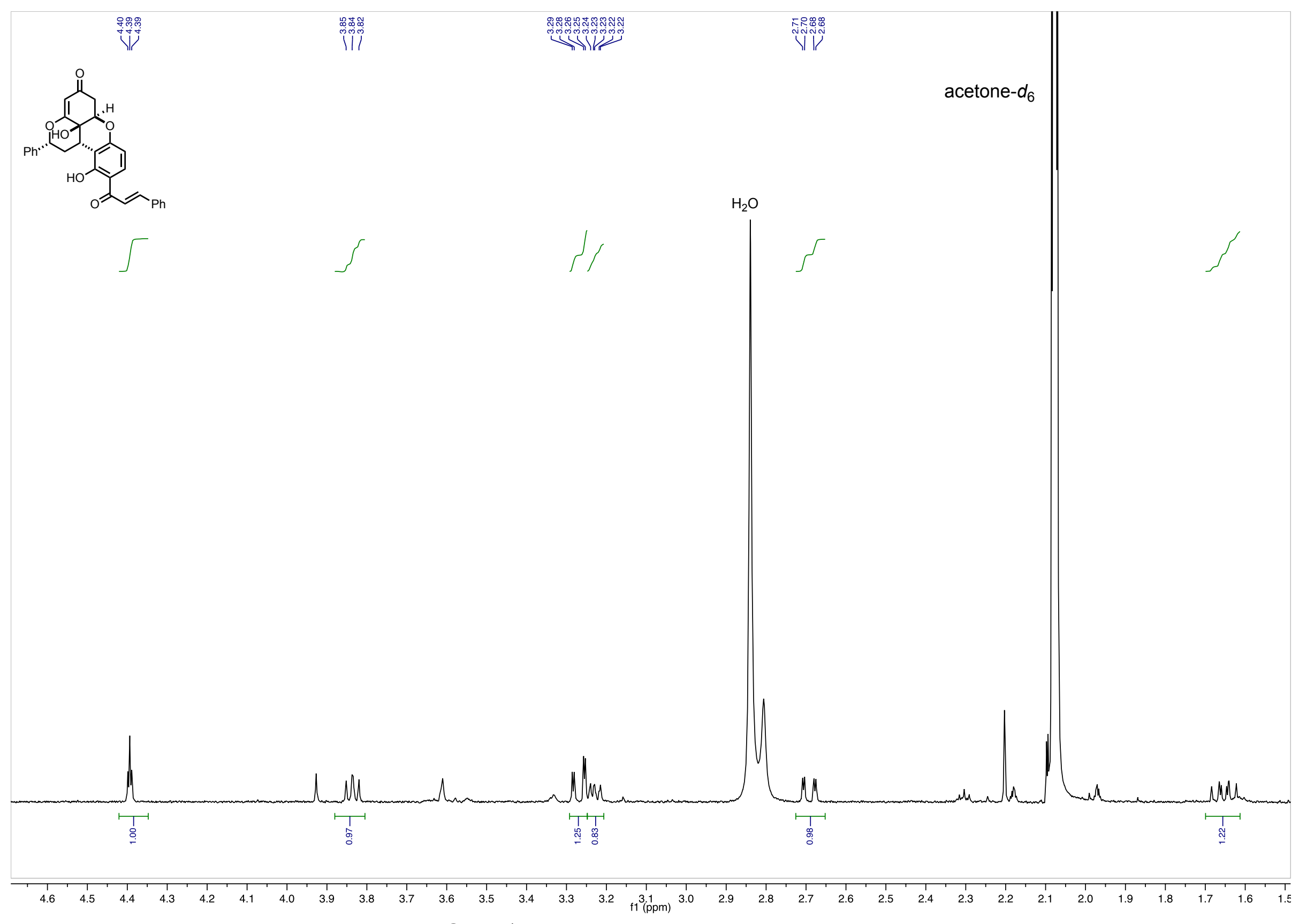

Figure S116. ${ }^{1} \mathrm{H}$ NMR (600 MHz, acetone- $\left.d_{6}\right), 21$ (inset). 

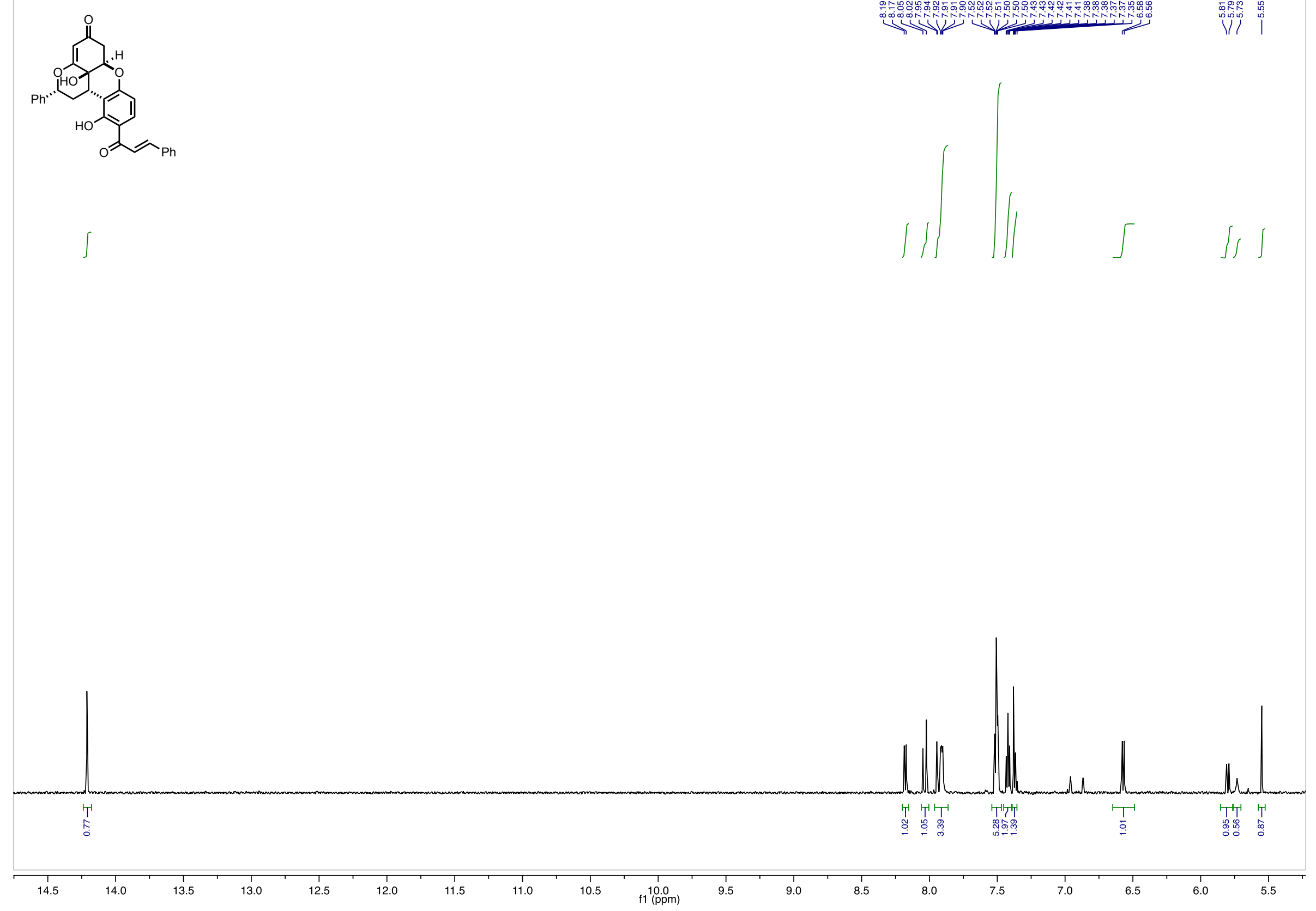

Figure S117. ${ }^{1} \mathrm{H}$ NMR (600 MHz, acetone-d6), 21 (inset). 


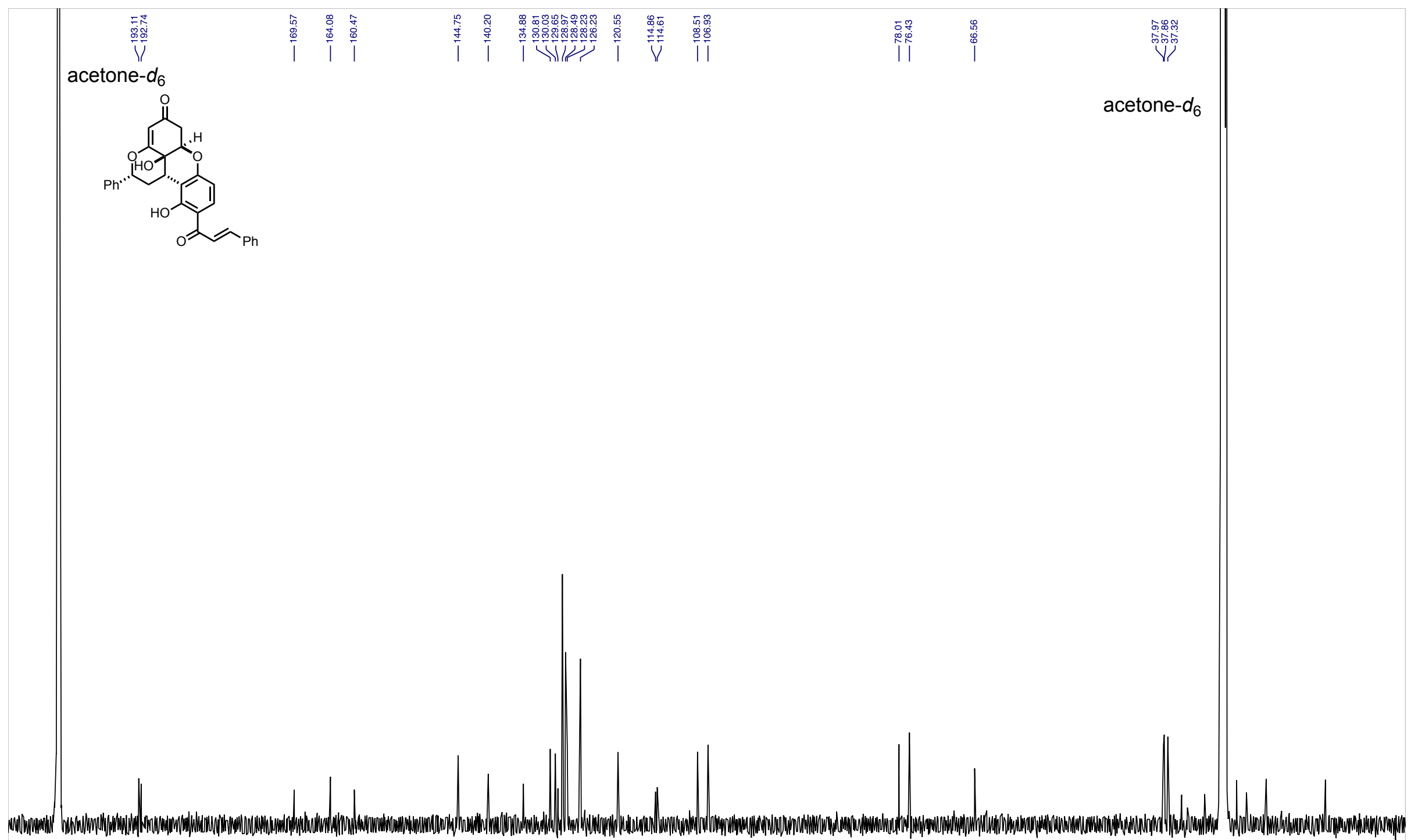

Figure S118. ${ }^{13} \mathrm{C}$ NMR (151 MHz, acetone- $\left.d_{6}\right), 21$. 


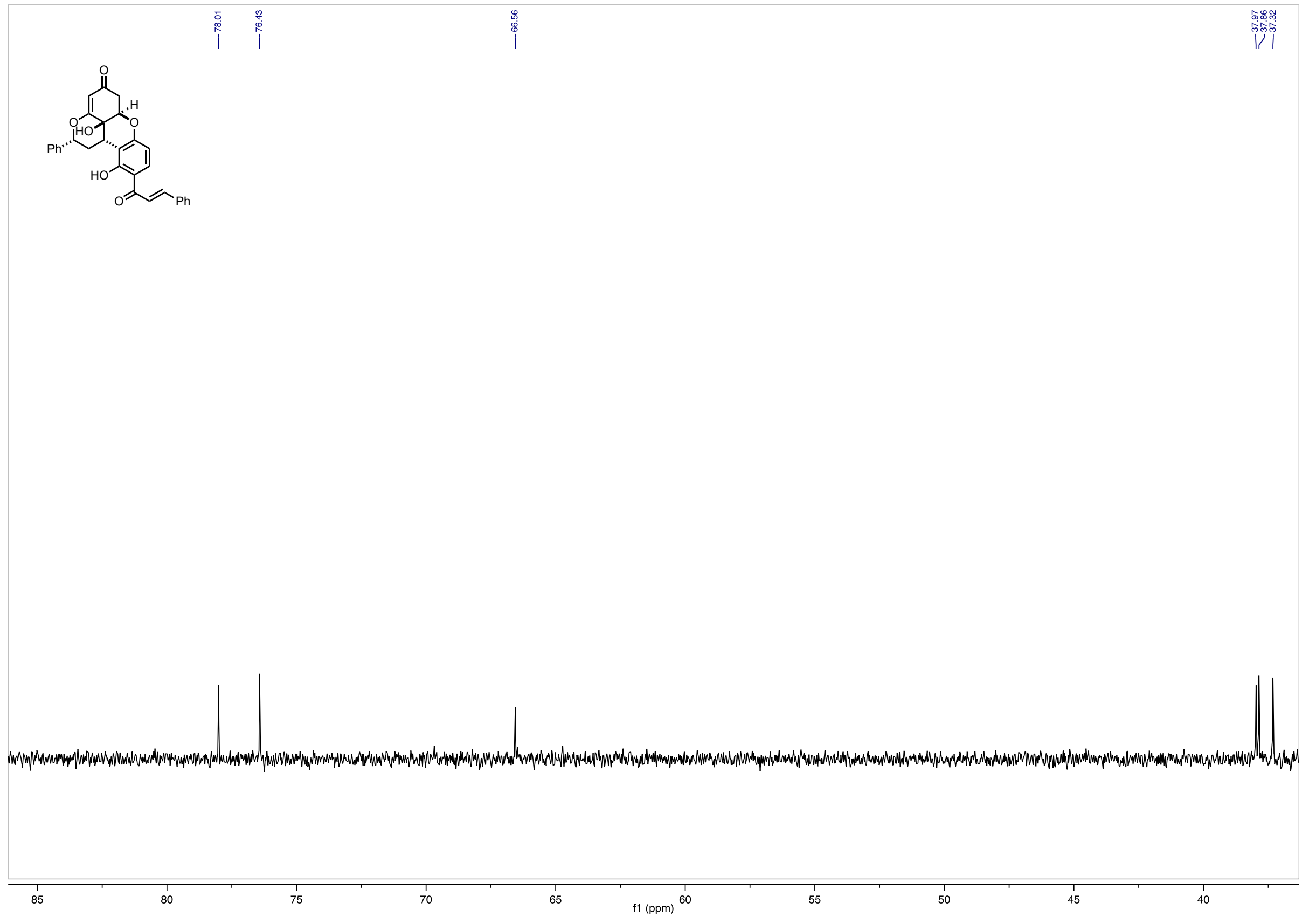

Figure S119. ${ }^{13} \mathrm{C}$ NMR (151 MHz, acetone-d6), 21 (inset). 

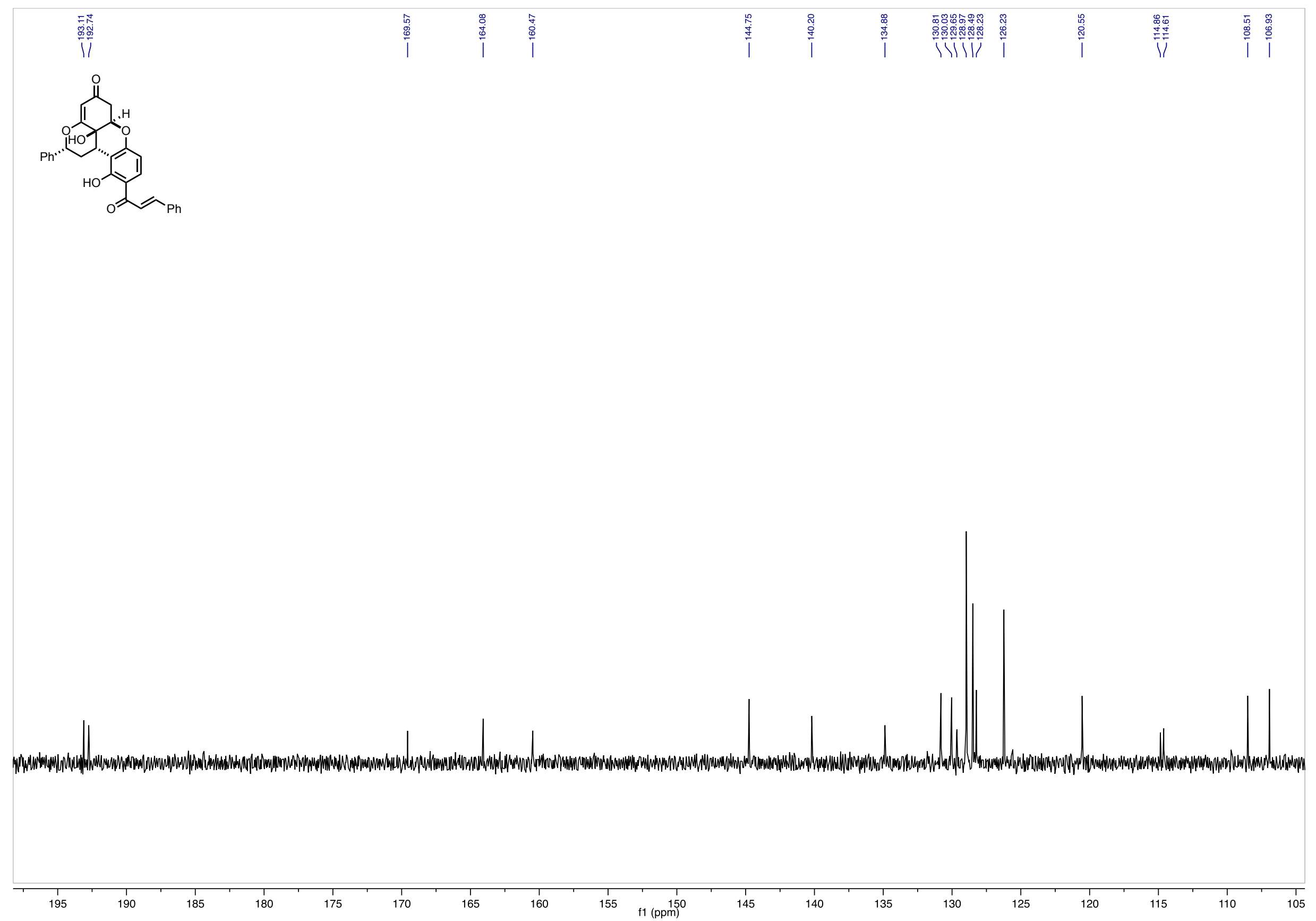

Figure $\mathbf{S 1 2 0} .{ }^{13} \mathrm{C}$ NMR (151 MHz, acetone- $\left.d_{6}\right), 21$ (inset). 


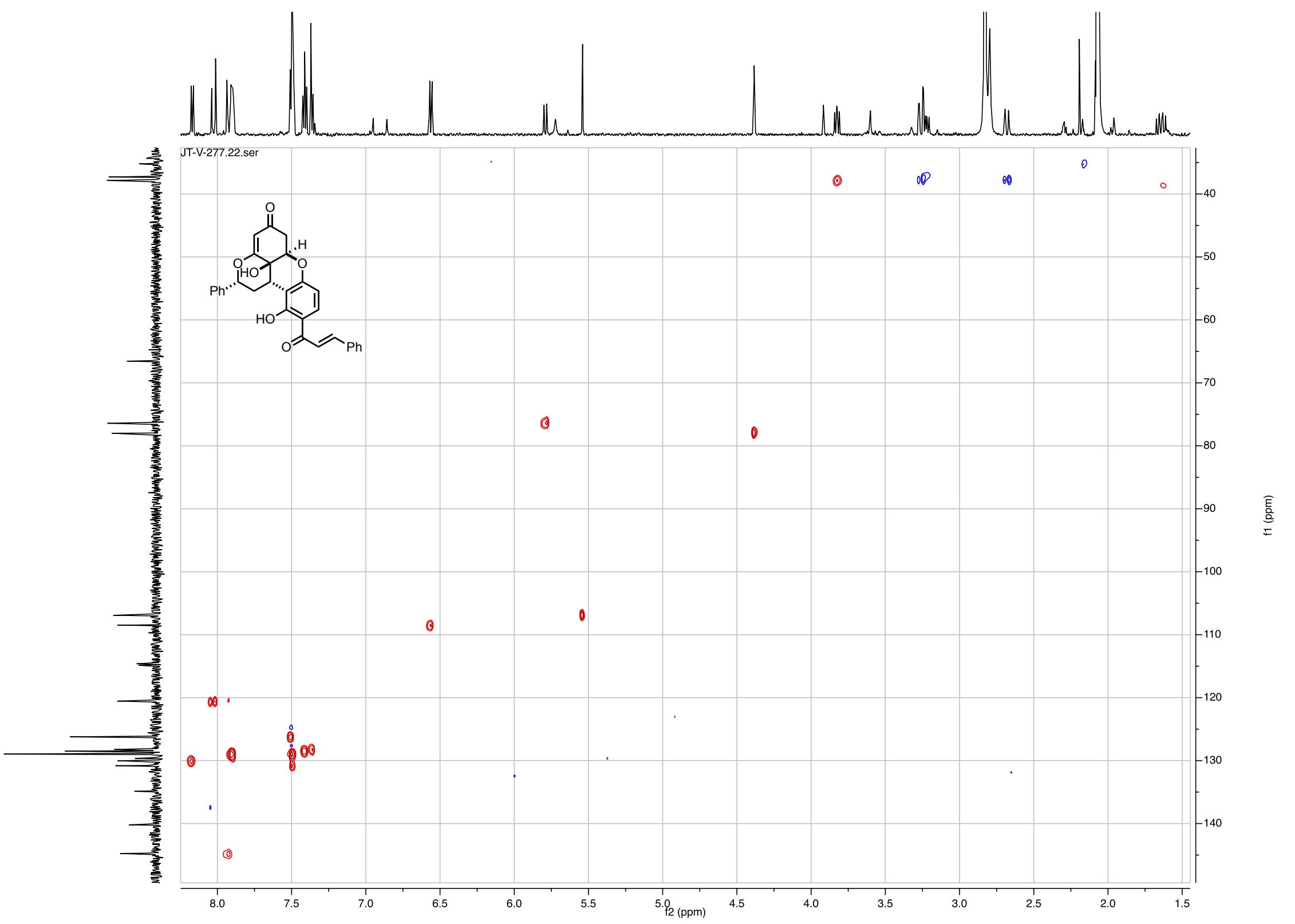

Figure S121. ${ }^{1} \mathrm{H}-{ }^{13} \mathrm{C}$ HSQC spectrum, 21. 


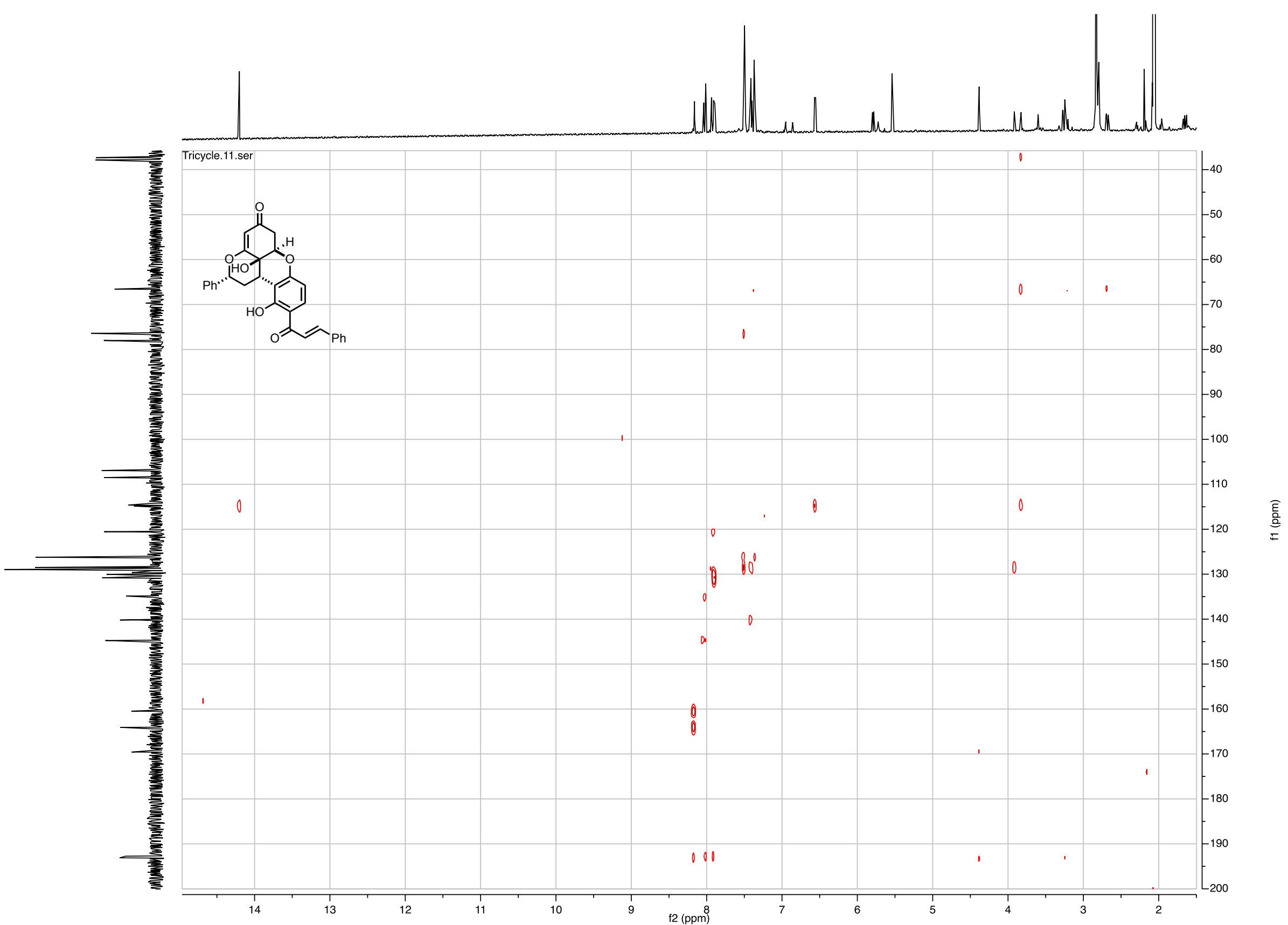

Figure S122. ${ }^{1} \mathrm{H}-{ }^{13} \mathrm{C}$ HMBC spectrum, 21.

S178 


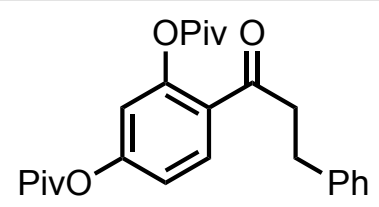

$\mathrm{PivO}_{\mathrm{Ph}}$

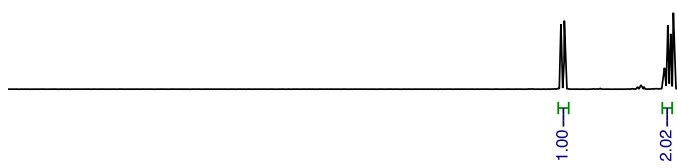

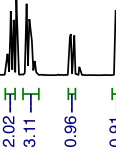

Figure S123. ${ }^{1} \mathrm{H}$ NMR (600 MHz, chloroform-d), SI-2. 


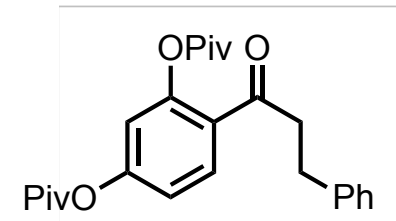

or

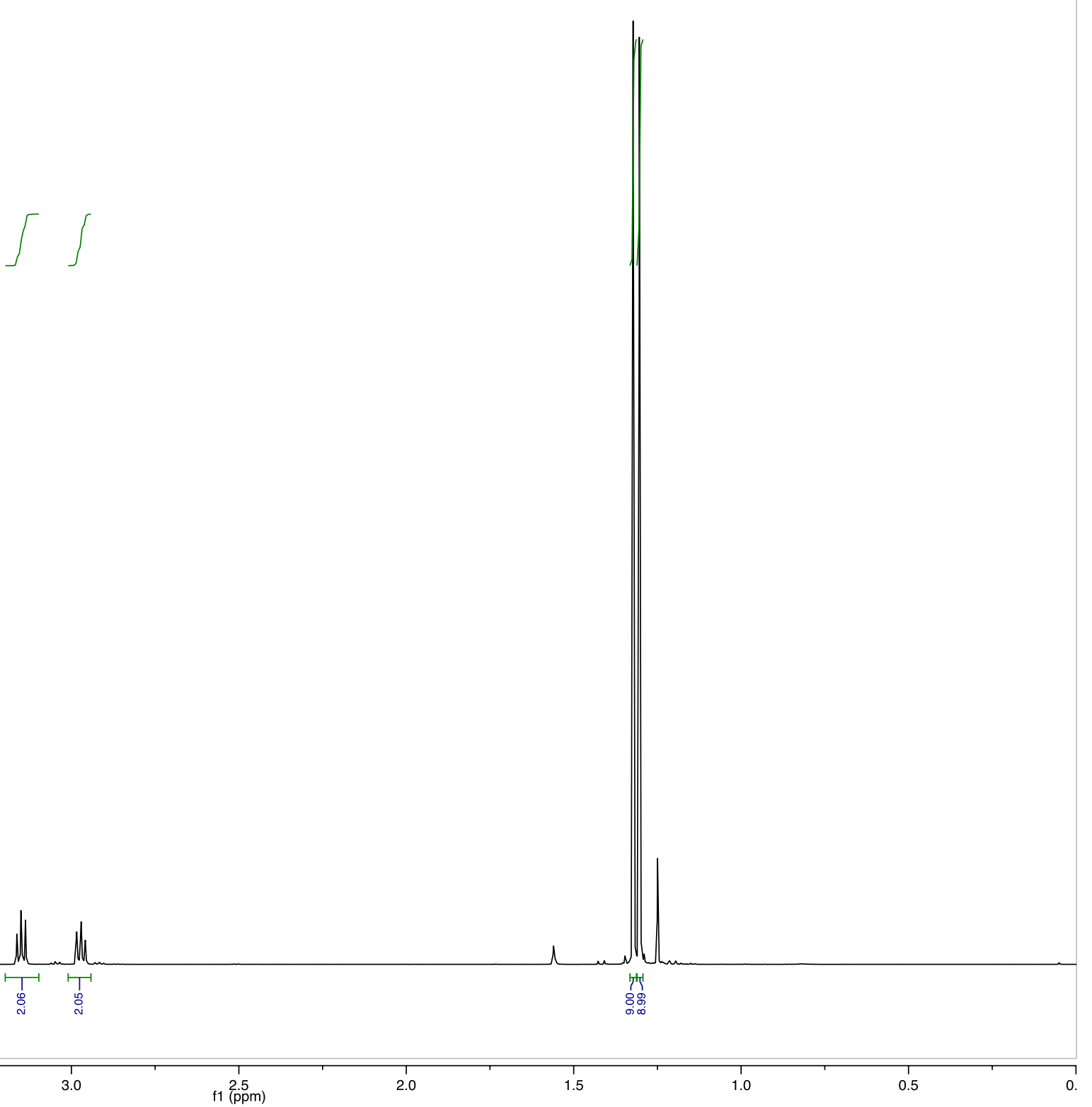

Figure S124. ${ }^{1} \mathrm{H}$ NMR (600 MHz, chloroform- $d$ ), SI-2 (inset). 

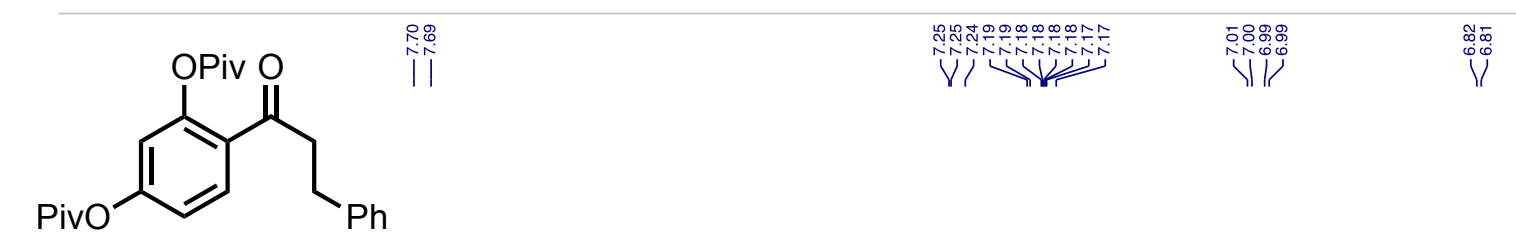

$\mathrm{PivO}_{\mathrm{Ph}}$

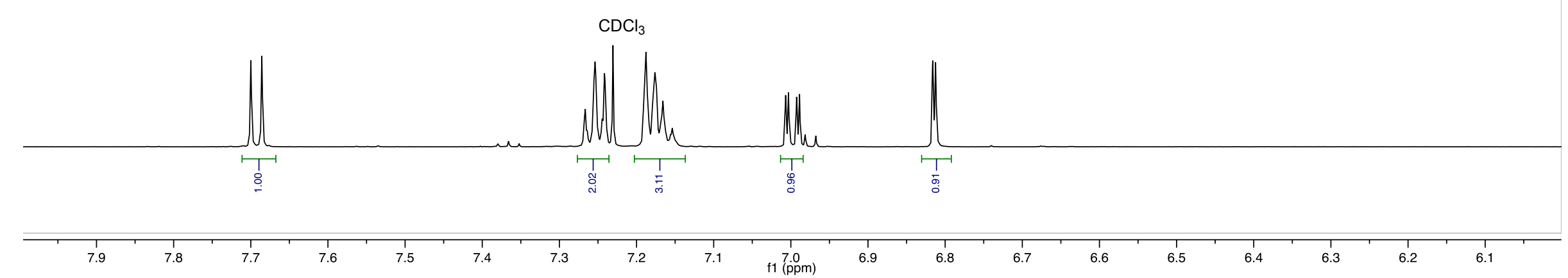

Figure S125. ${ }^{1} \mathrm{H}$ NMR (600 MHz, chloroform- $d$ ), SI-2 (inset). 


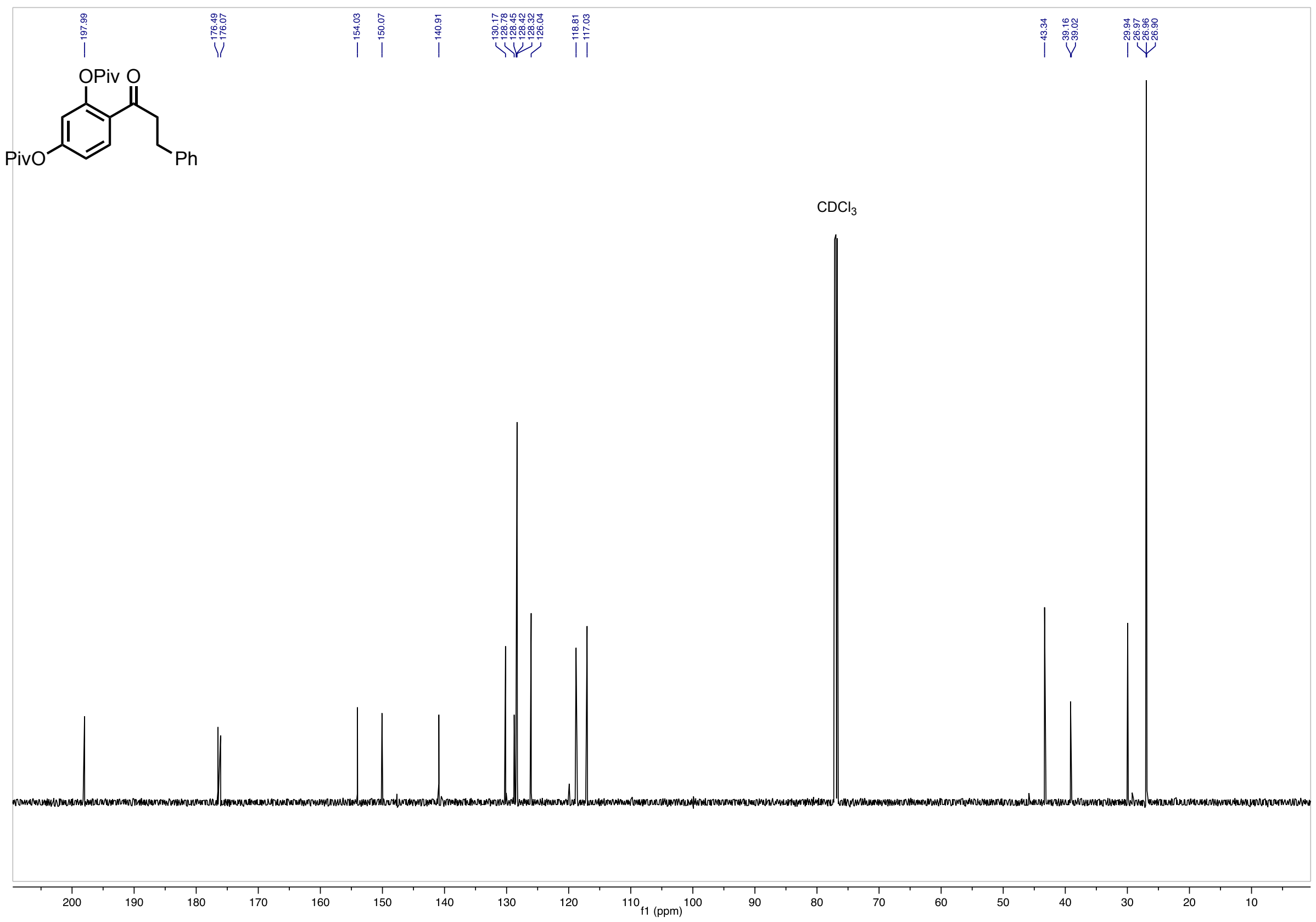

Figure S126. ${ }^{13} \mathrm{C}$ NMR (151 MHz, chloroform- $d$ ), SI-2.

S182 


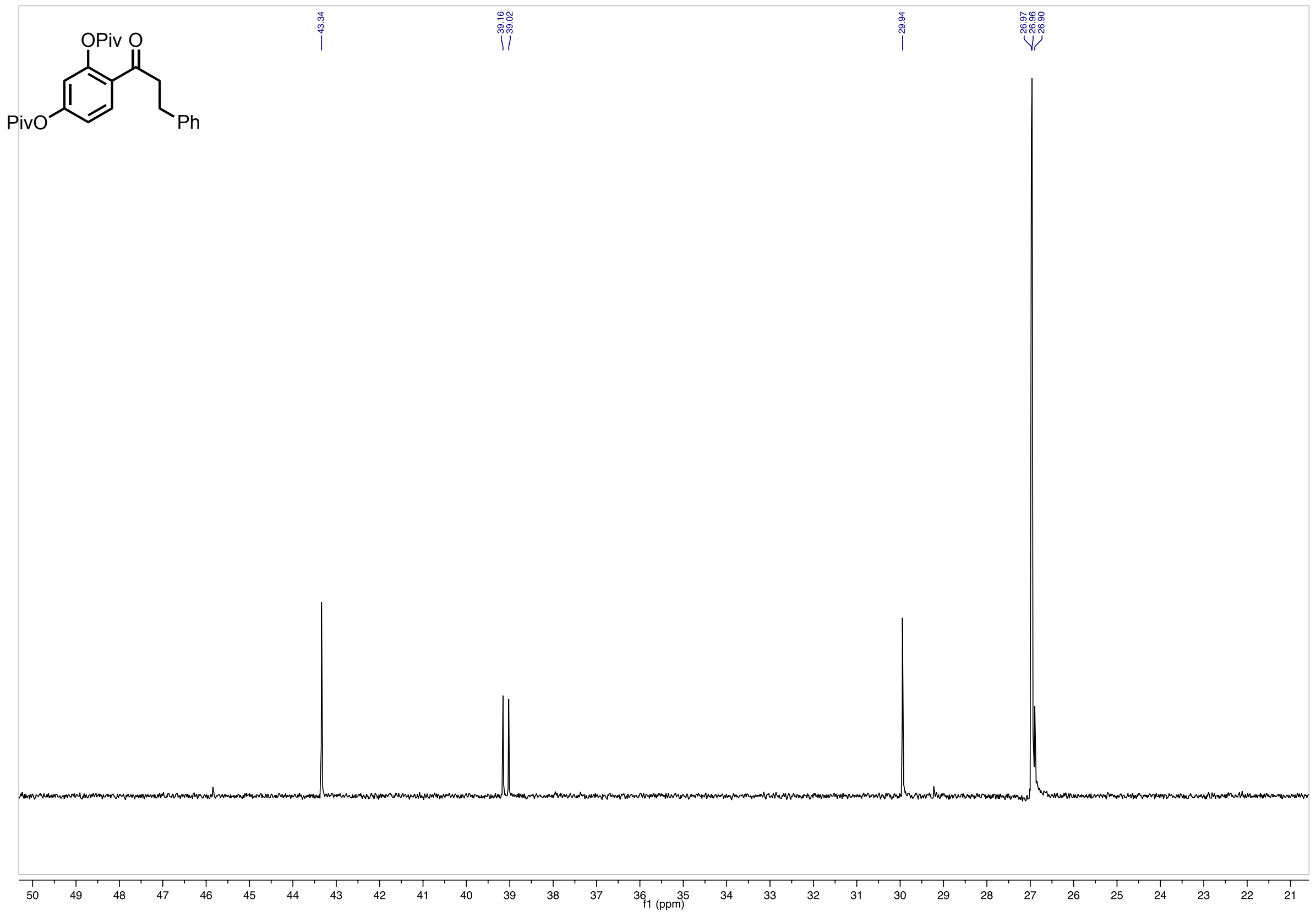

Figure S127. ${ }^{13} \mathrm{C}$ NMR (151 MHz, chloroform-d), SI-2 (inset).

S183 


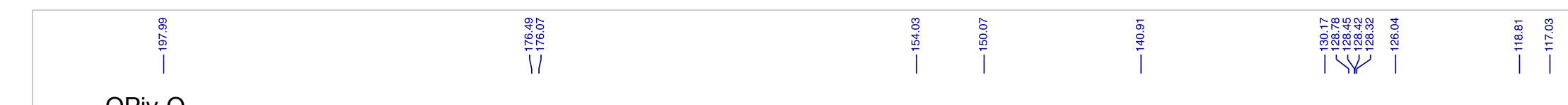
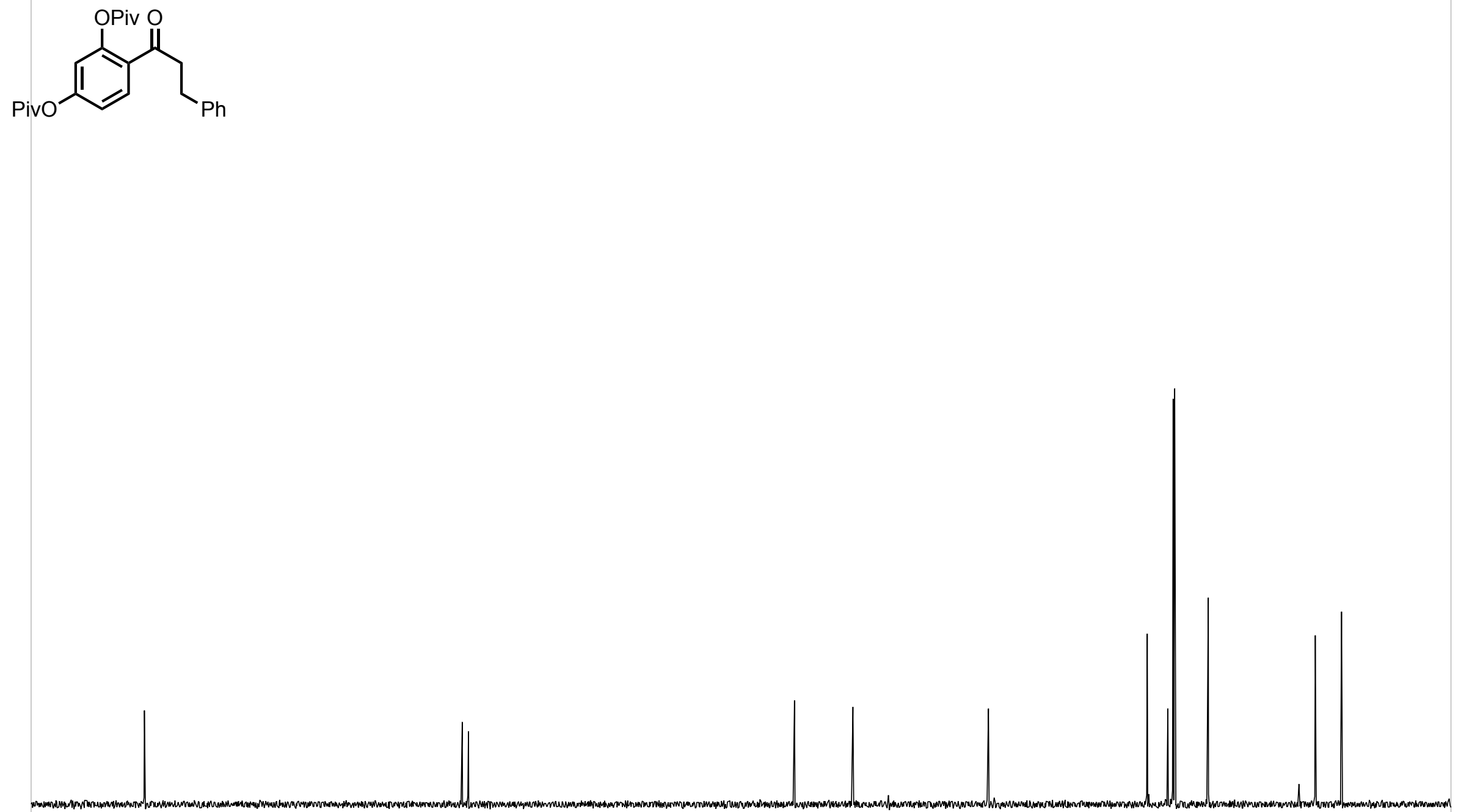$$
\text { T1 }
$$

Figure S128. ${ }^{13} \mathrm{C}$ NMR (151 MHz, chloroform-d), SI-2 (inset). 


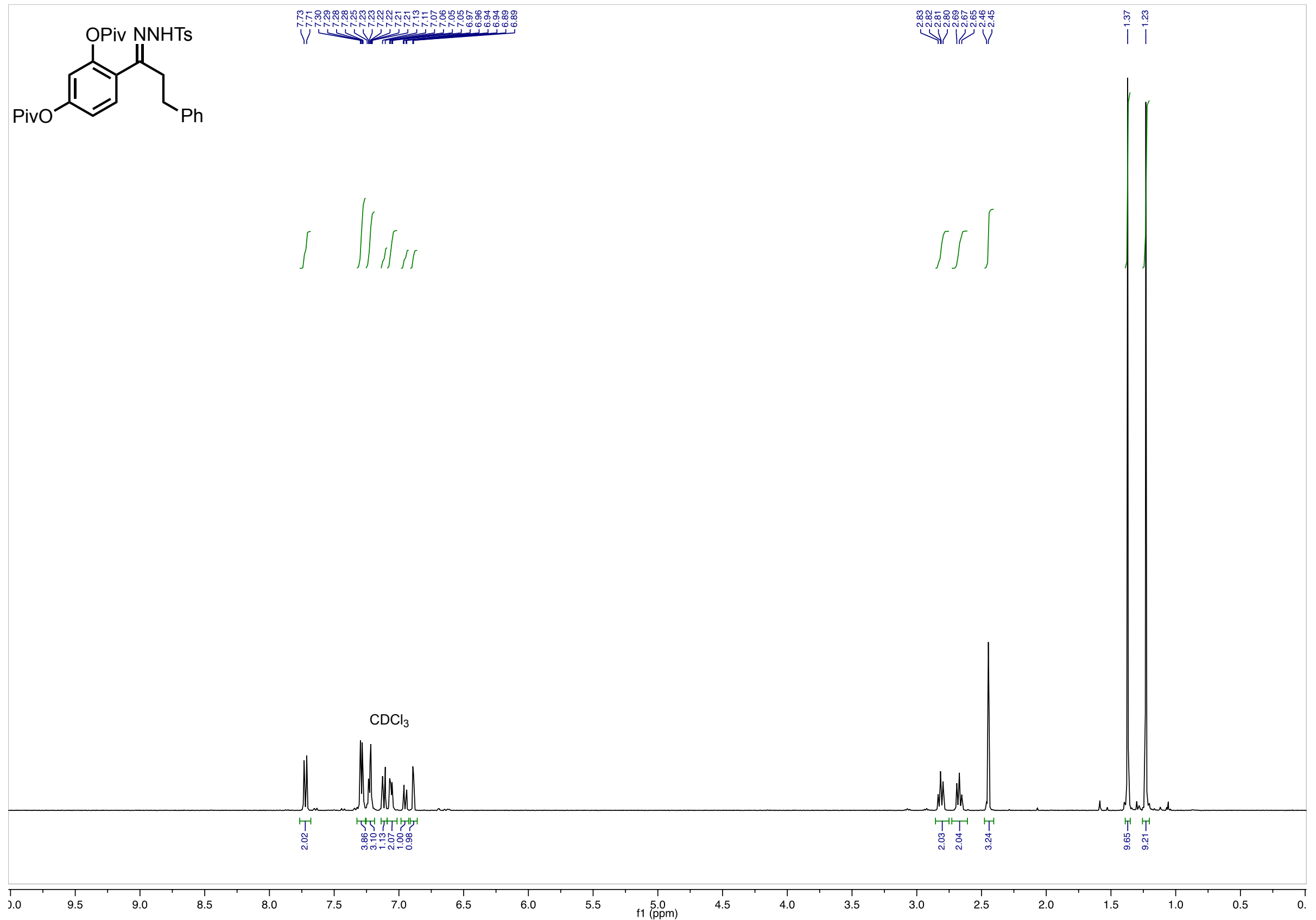

Figure S129. ' ${ }^{1 H}$ NMR (600 MHz, chloroform-d), SI-3. 


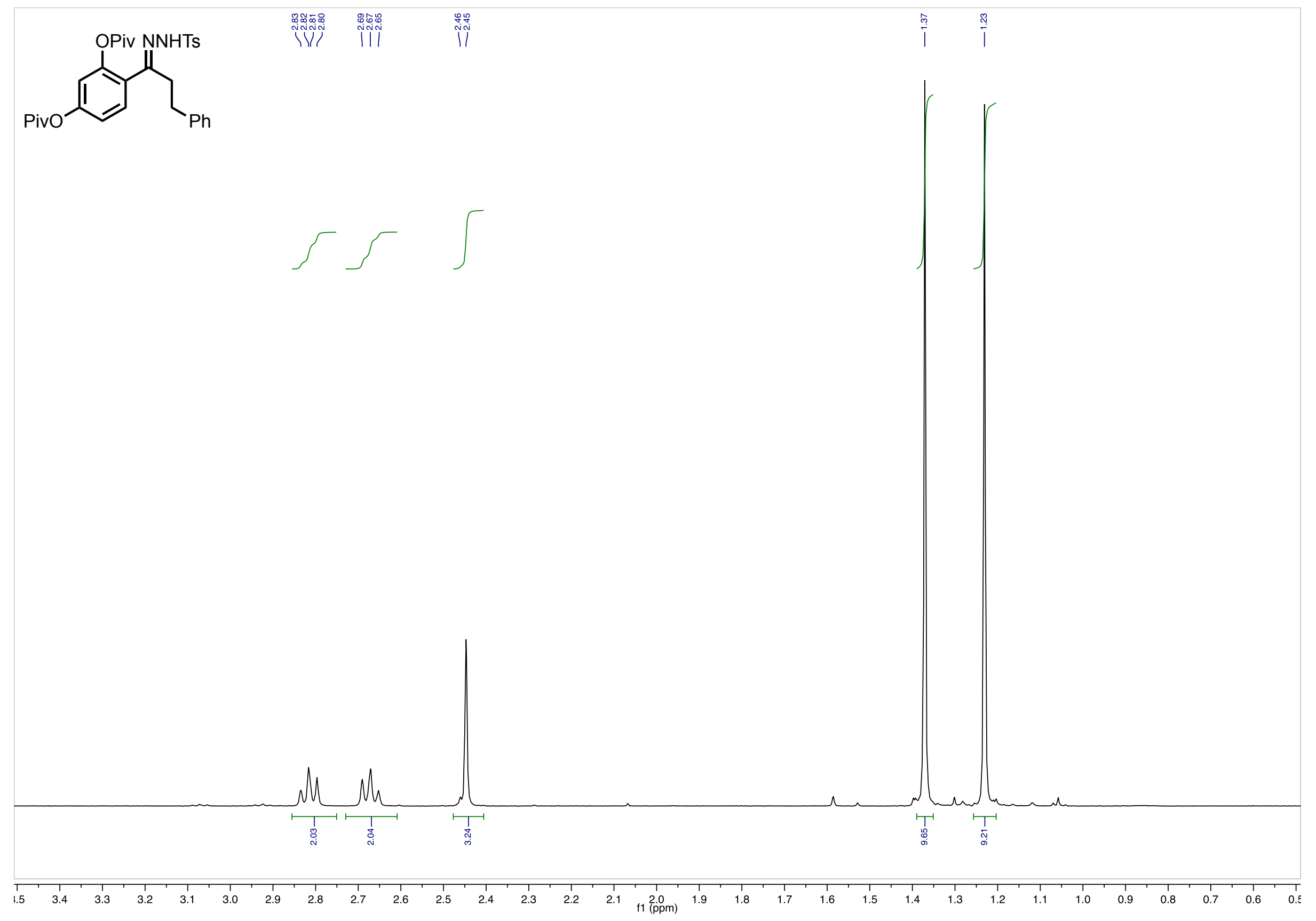

Figure S130. ${ }^{1} \mathrm{H}$ NMR (600 MHz, chloroform- $d$ ), SI-3 (inset). 


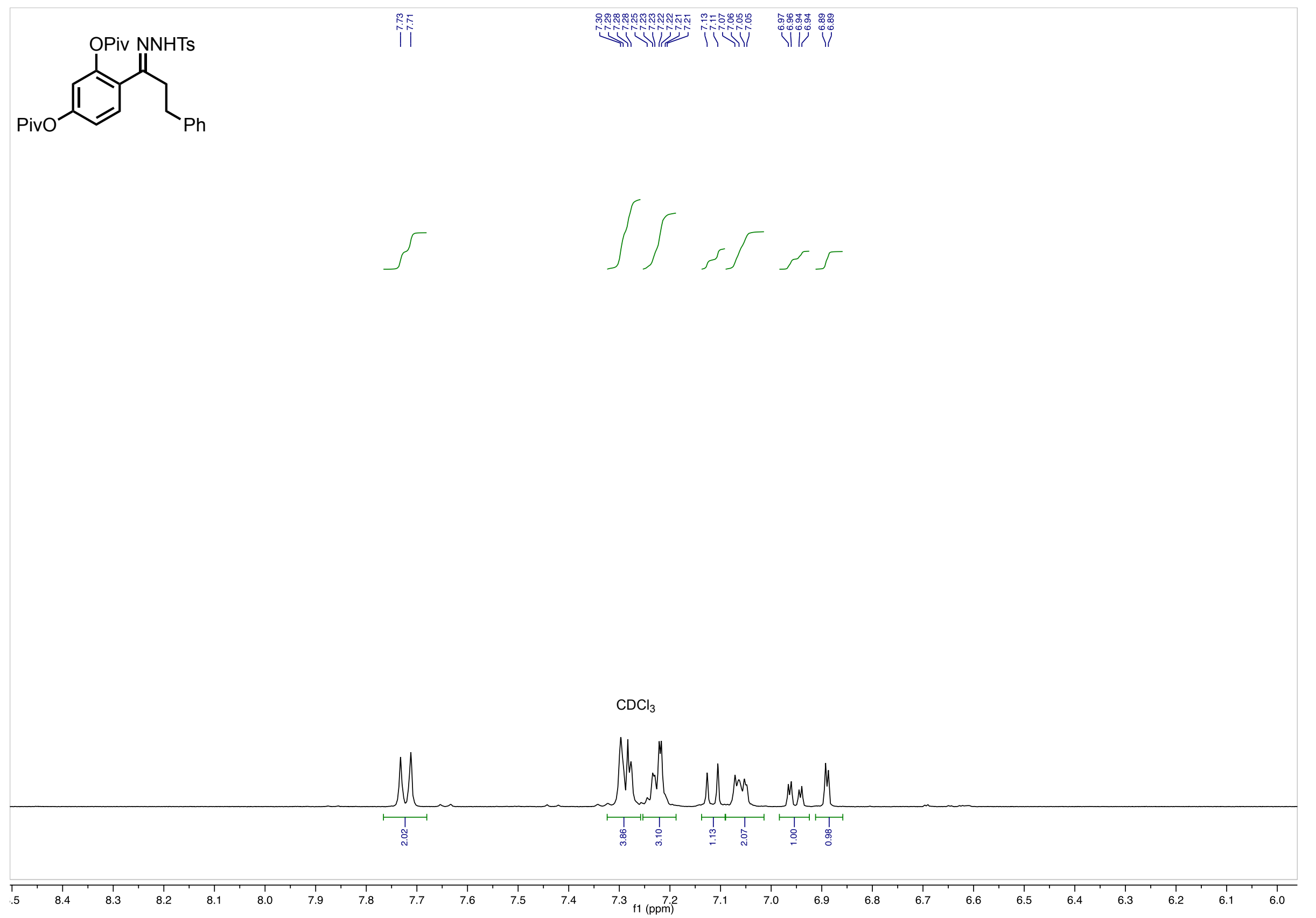

Figure S131. ${ }^{1} \mathrm{H}$ NMR (600 MHz, chloroform-d), SI-3 (inset). 


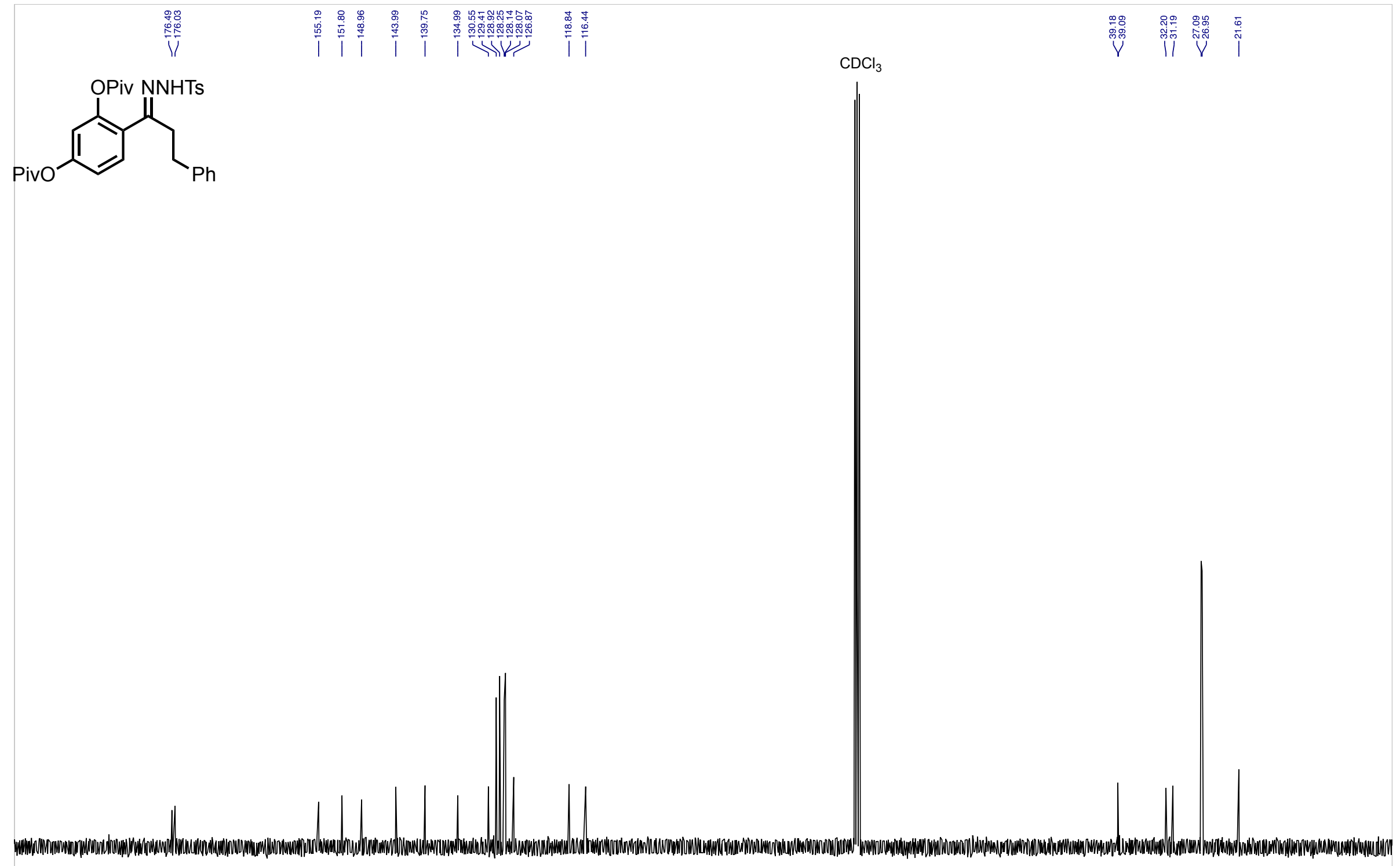

190

180

170

160

150 120 110

$\mathrm{f1}^{100}(\mathrm{ppm})$

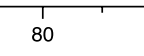

70

60

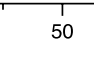

Figure S132. ${ }^{13} \mathrm{C}$ NMR (151 MHz, chloroform- $d$ ), SI-3. 

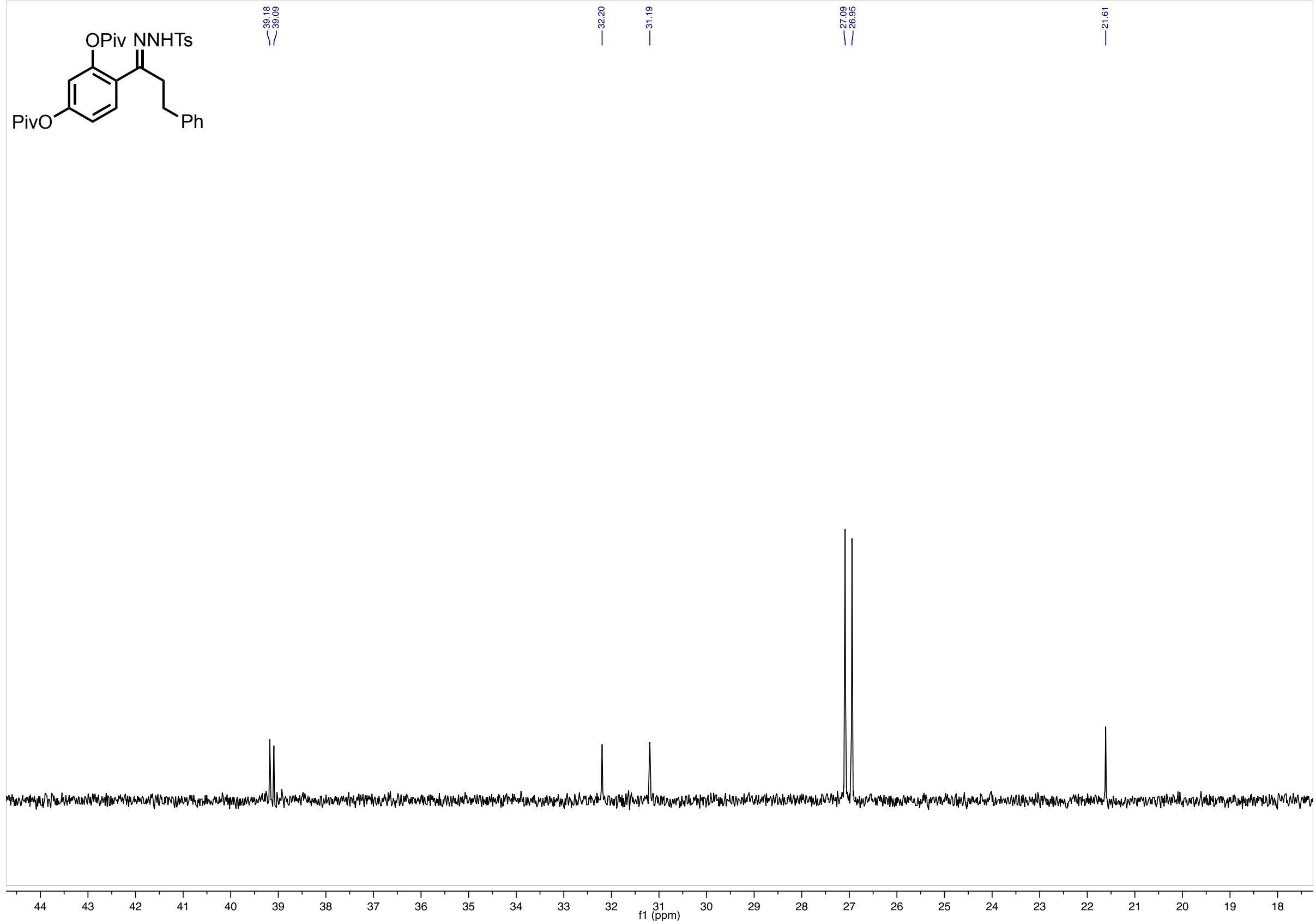

Figure S133. ${ }^{13} \mathrm{C}$ NMR (151 MHz, chloroform-d), SI-3 (inset). 


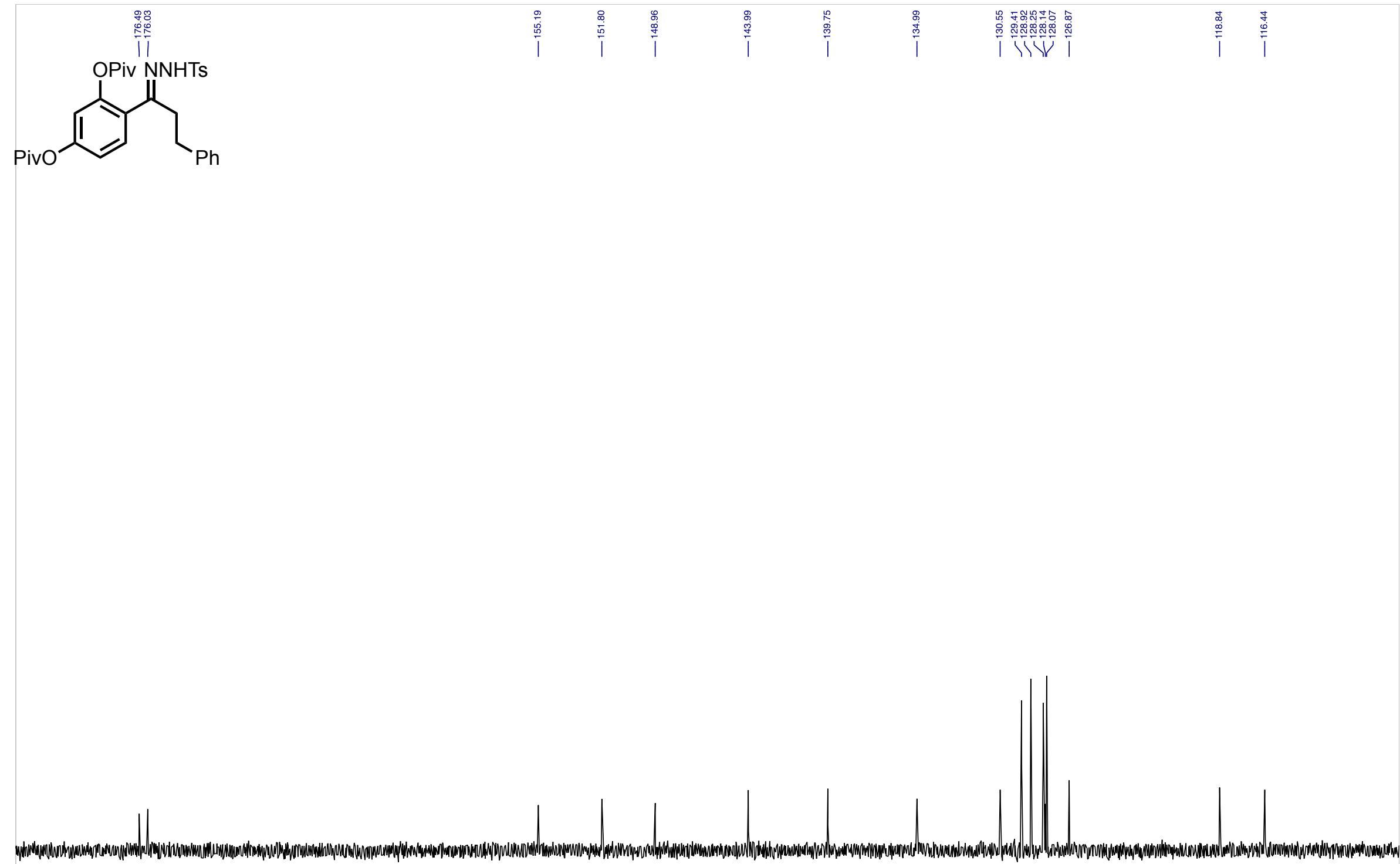

Figure S134. ${ }^{13} \mathrm{C}$ NMR (151 MHz, chloroform- $d$ ), SI-3 (inset). 


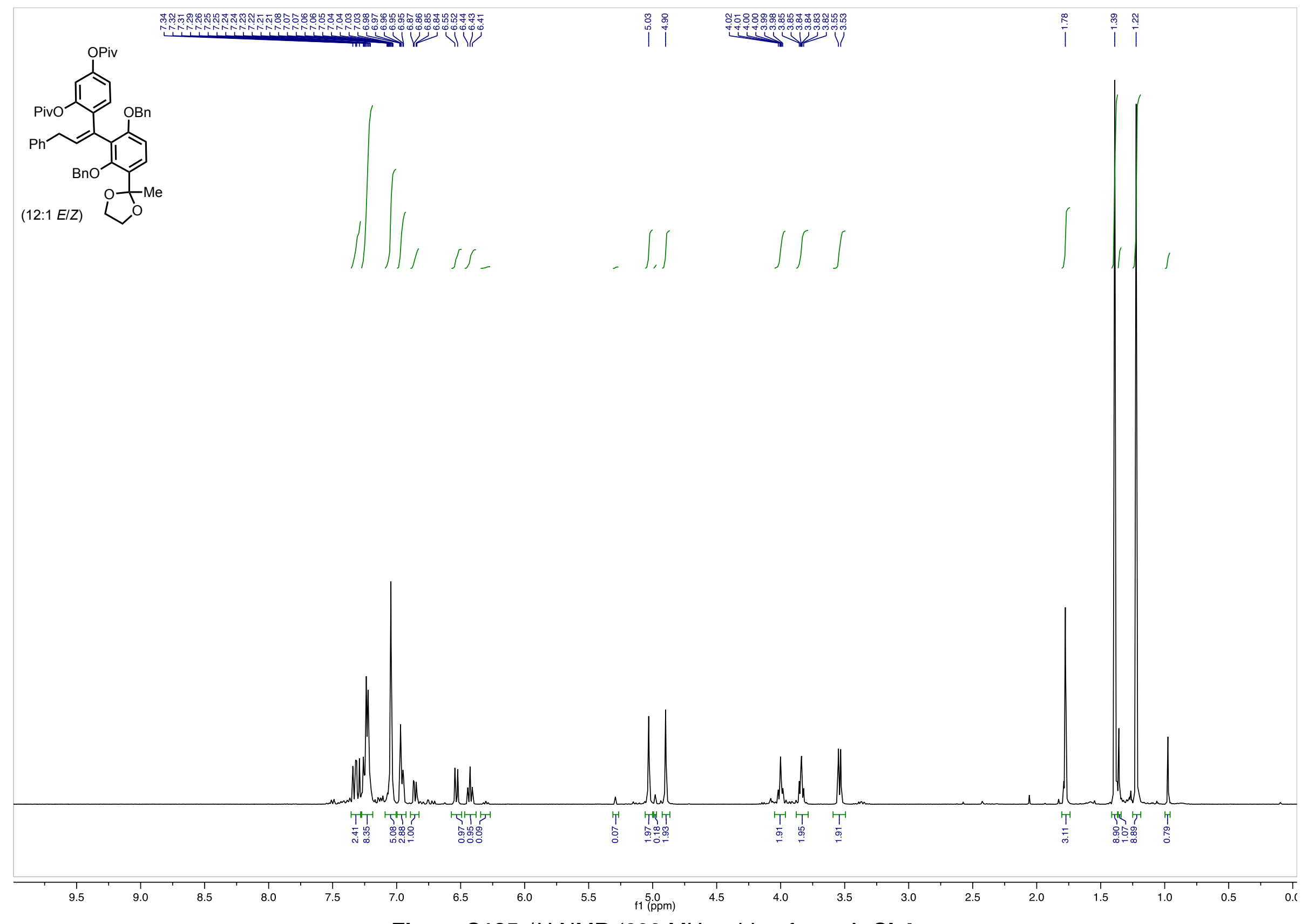

Figure S135. ${ }^{1} \mathrm{H}$ NMR (600 MHz, chloroform-d), SI-4. 


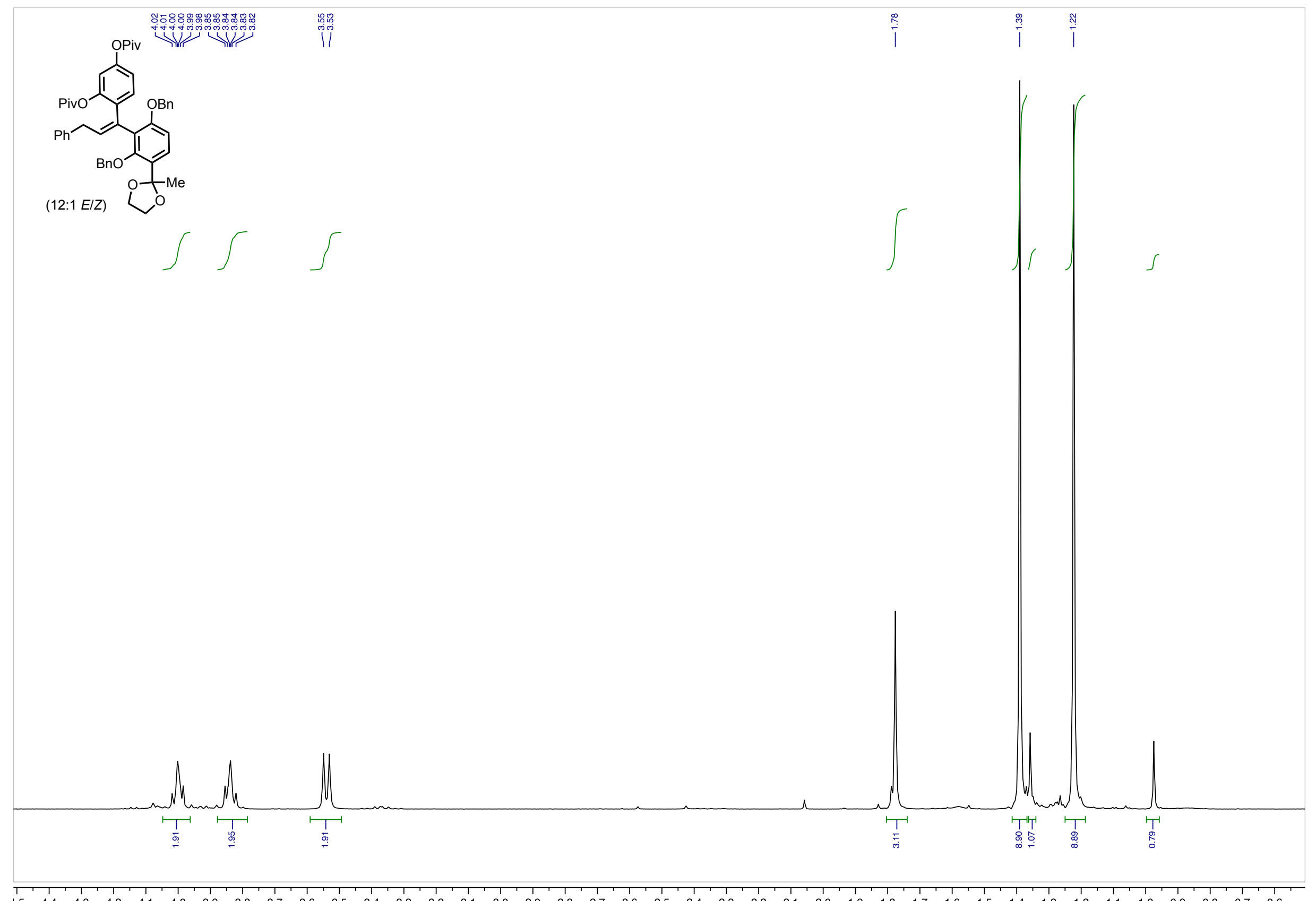

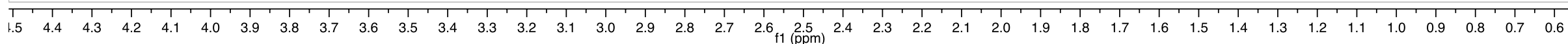

Figure S136. ${ }^{1} \mathrm{H}$ NMR (600 MHz, chloroform- $d$ ), SI-4 (inset). 


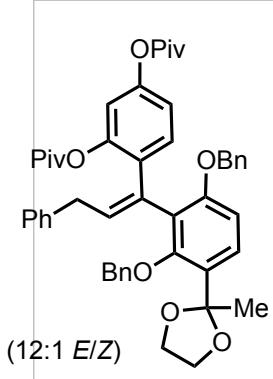

$(12: 1 E / Z)$
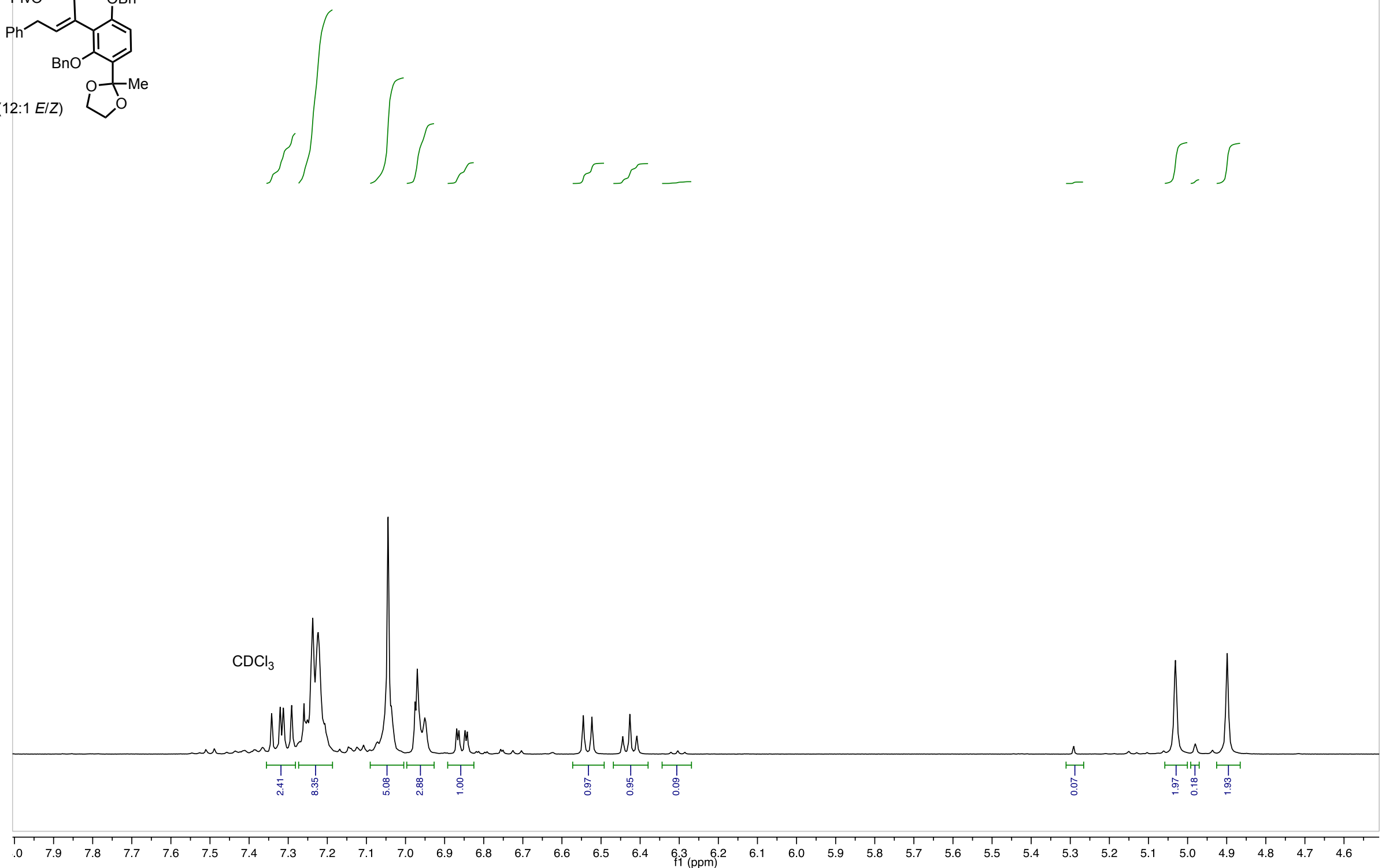

Figure S137. ${ }^{1 H} \mathrm{HMR}(600 \mathrm{MHz}$, chloroform-d), SI-4 (inset). 

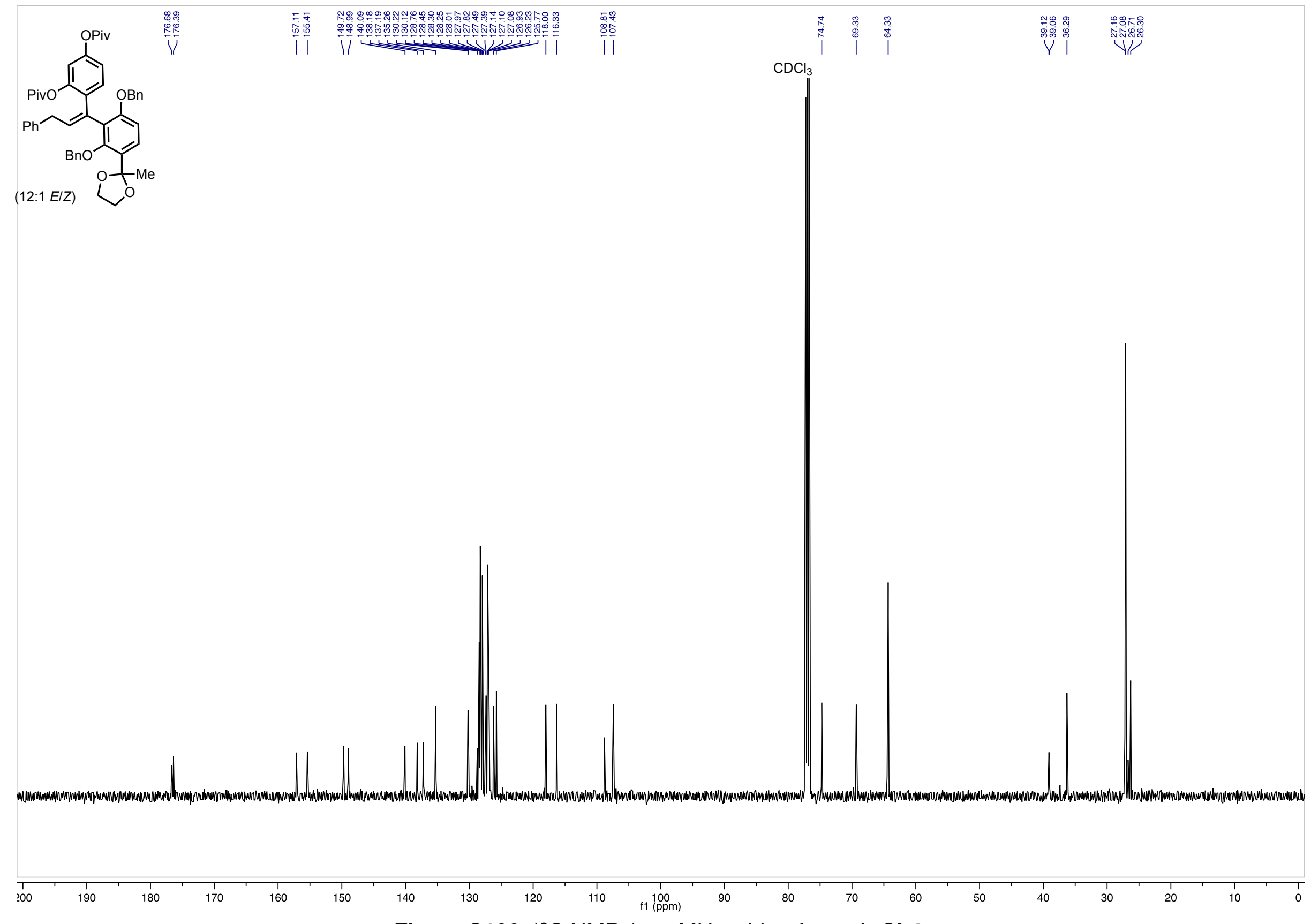

Figure S138. ${ }^{13} \mathrm{C}$ NMR (151 MHz, chloroform-d), SI-4. 


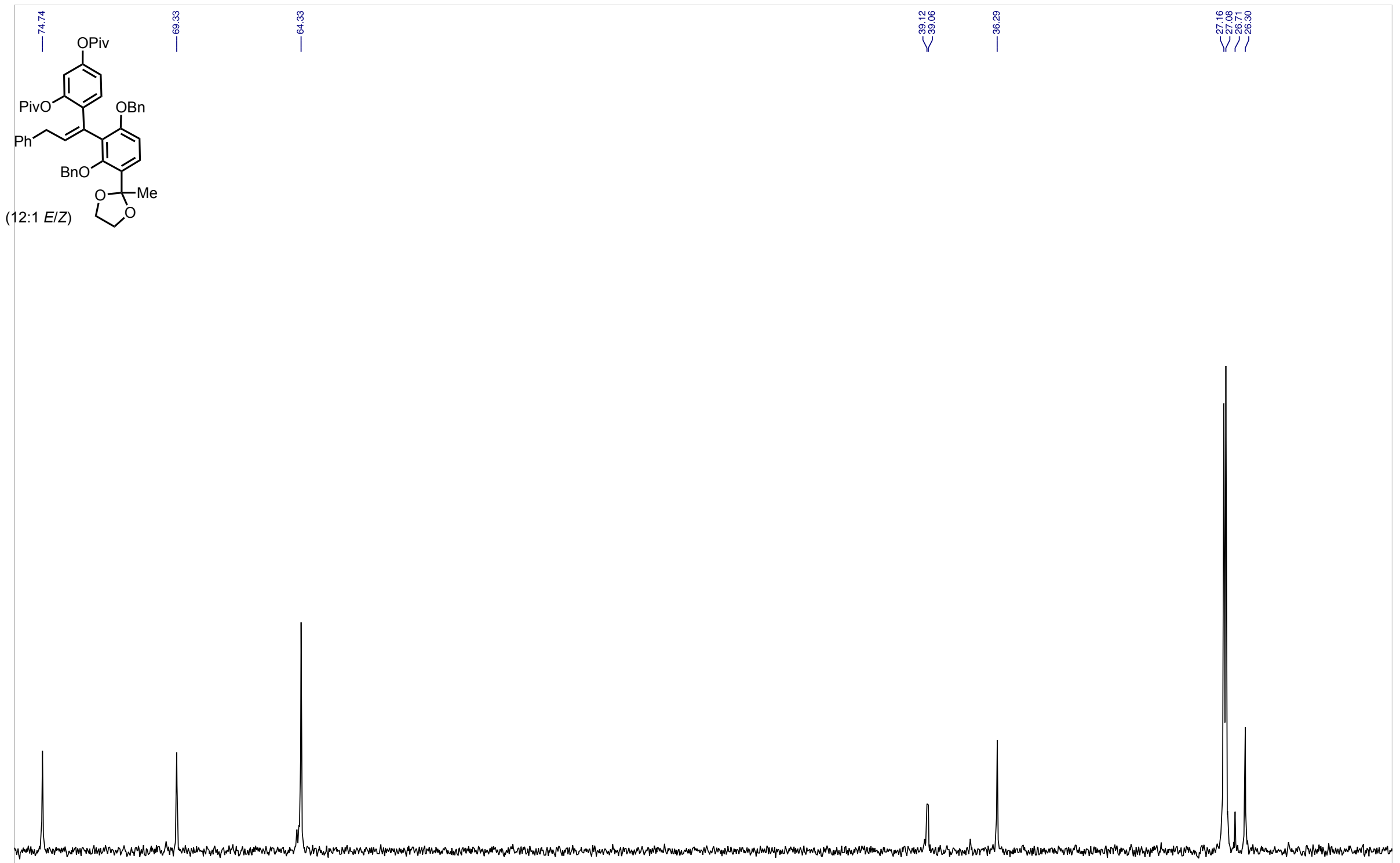

Figure S139. ${ }^{13} \mathrm{C}$ NMR (151 MHz, chloroform-d), SI-4 (inset). 


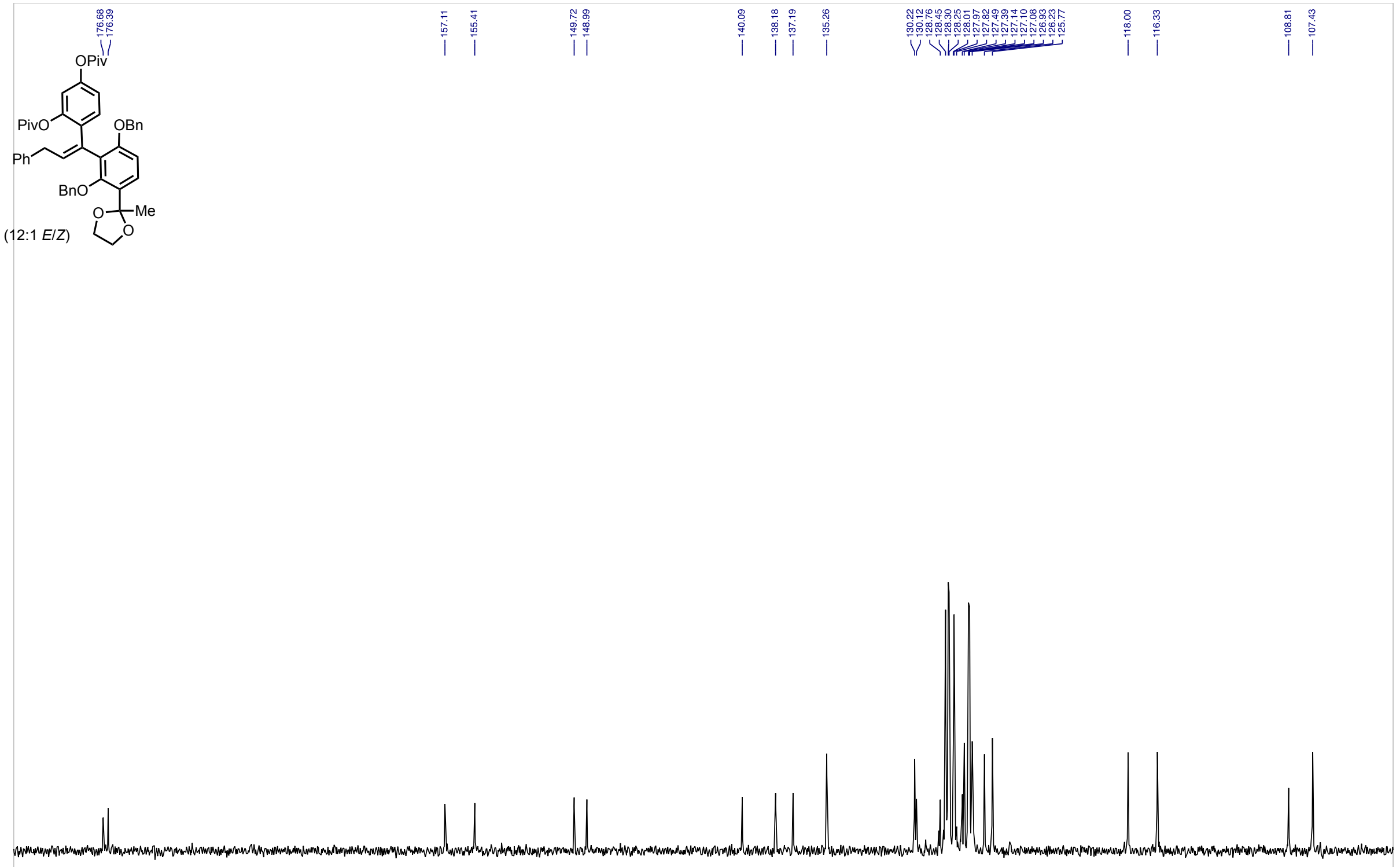

$145 \mathrm{f1}(\mathrm{ppm}) 140$

Figure S140. ${ }^{13} \mathrm{C}$ NMR (151 MHz, chloroform-d), SI-4 (inset). 


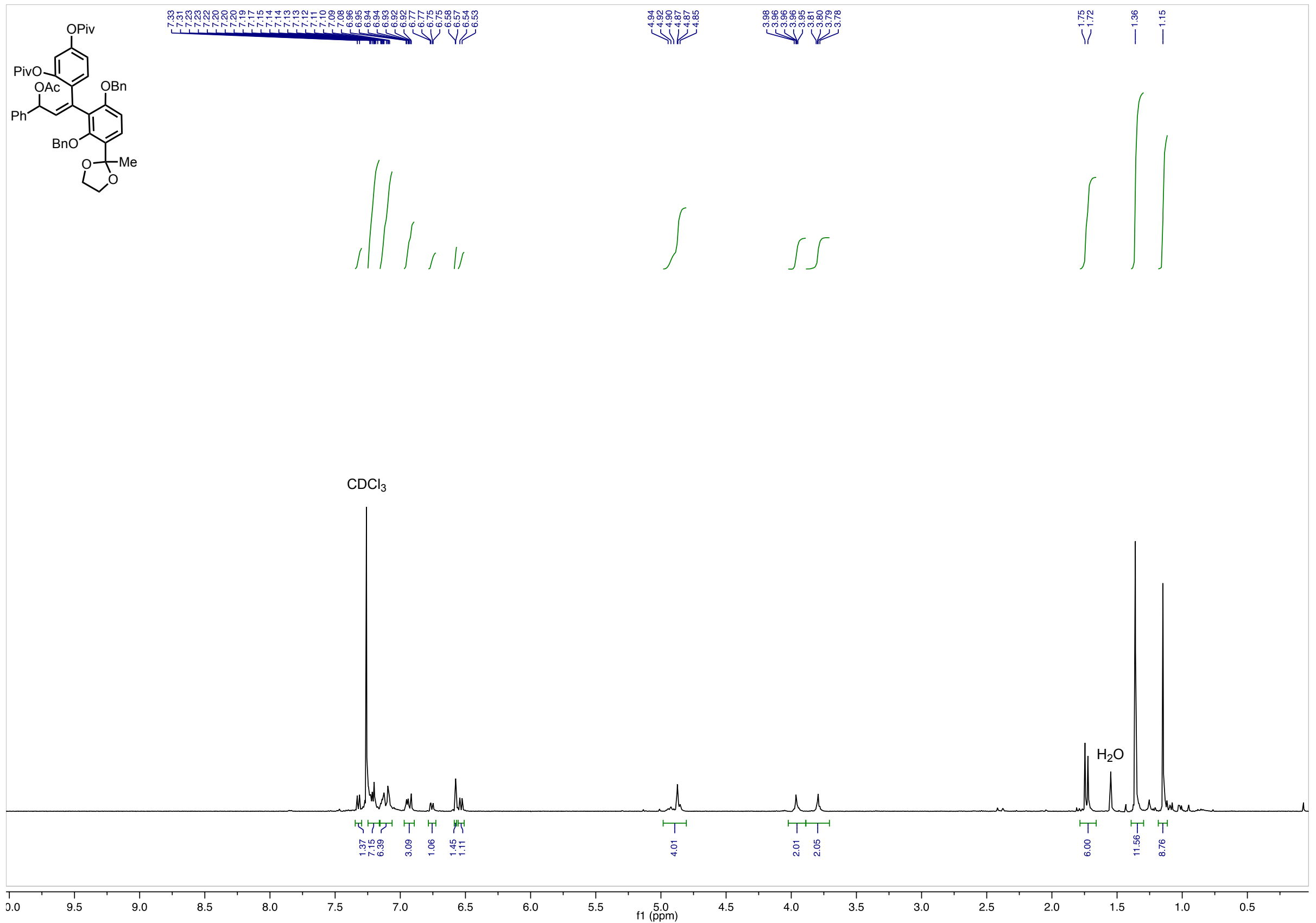

Figure S141. ${ }^{1} \mathrm{H}$ NMR (600 MHz, chloroform- $d$ ), SI-5. 


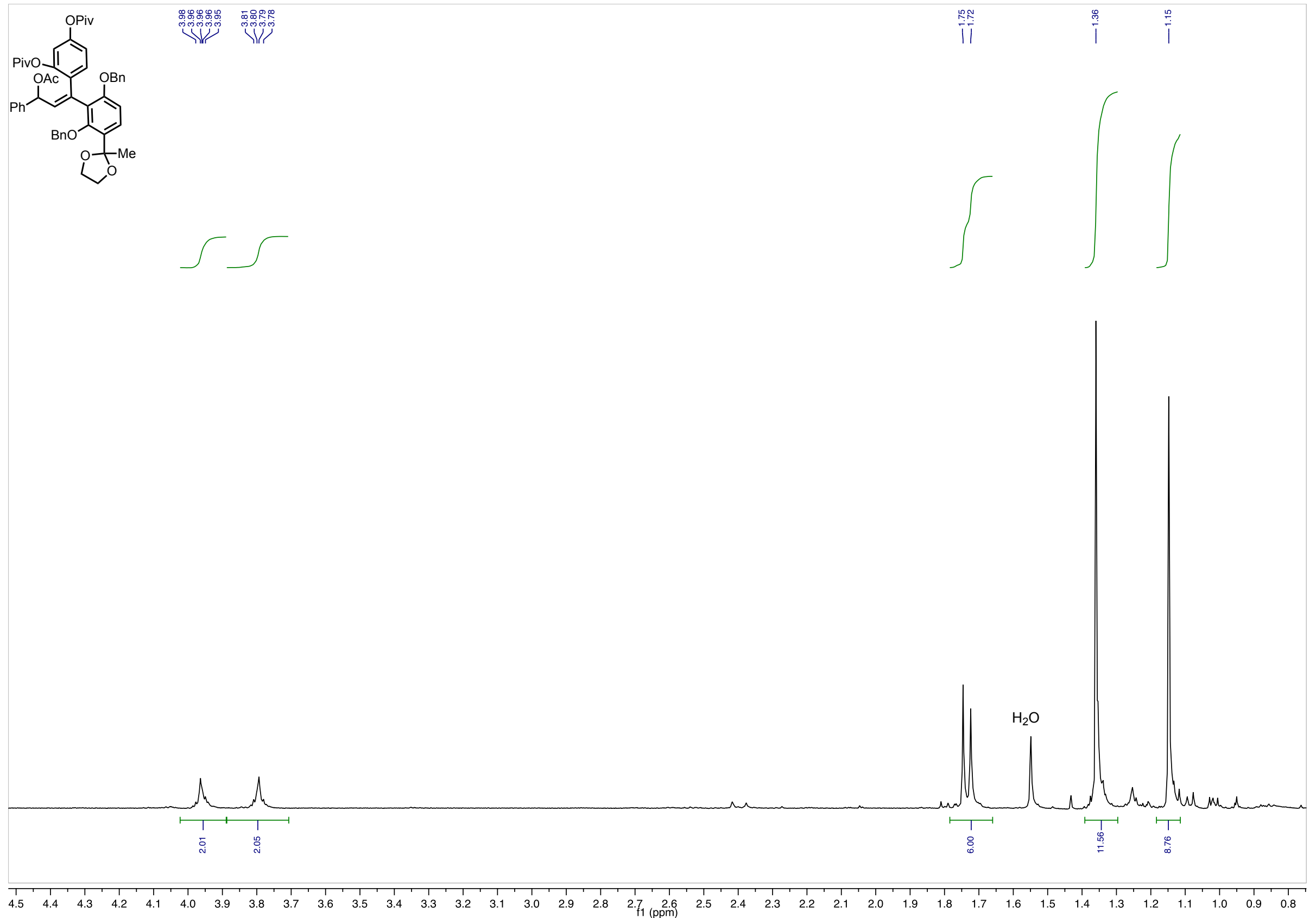

Figure S142. ${ }^{1} \mathrm{H}$ NMR (600 MHz, chloroform- $d$ ), SI-5 (inset). 


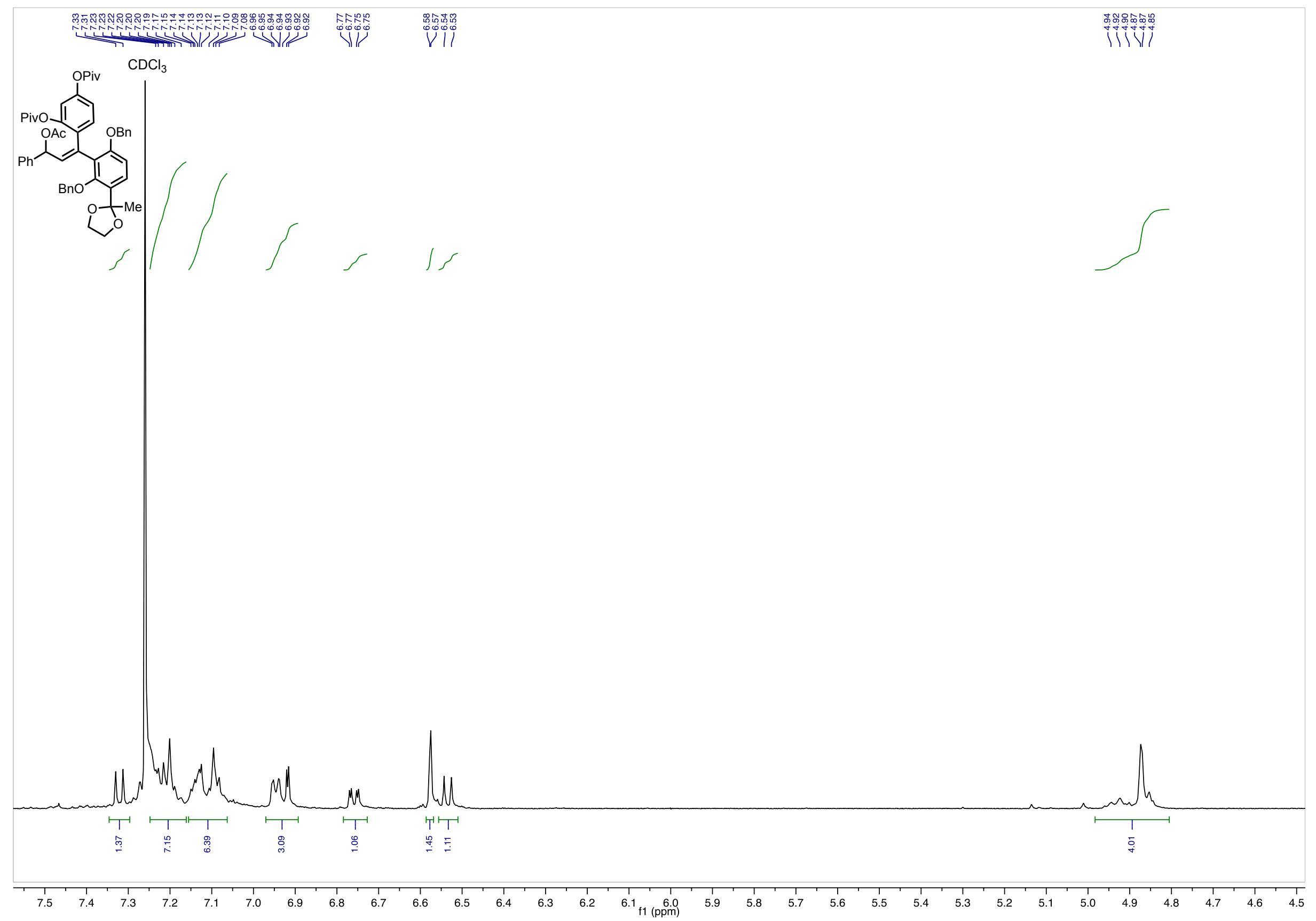

Figure S143. ${ }^{1} \mathrm{H}$ NMR (600 MHz, chloroform- $d$ ), SI-5 (inset). 


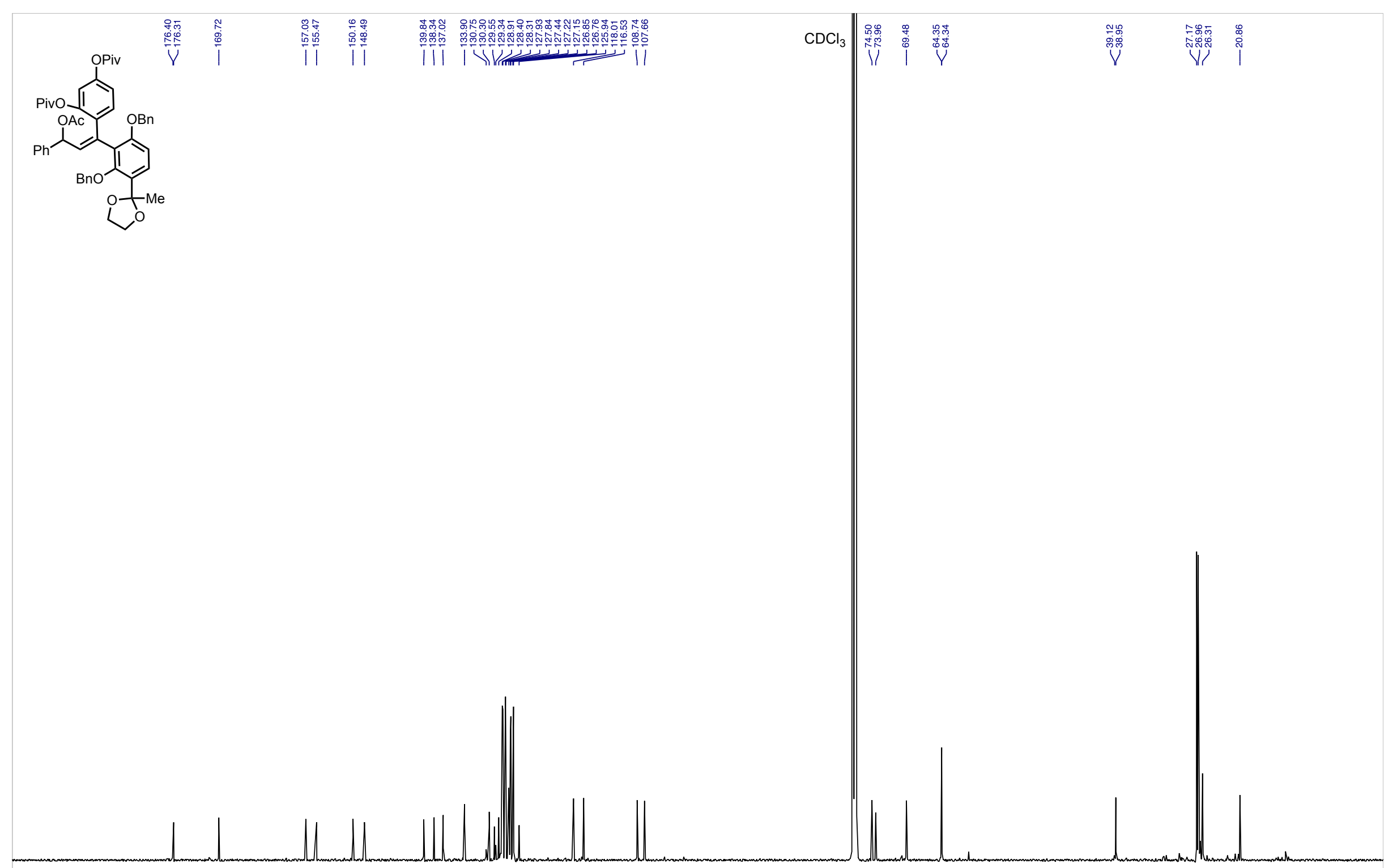

$190 \quad 180$

170
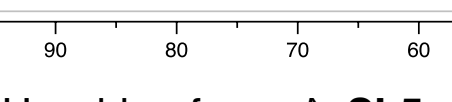

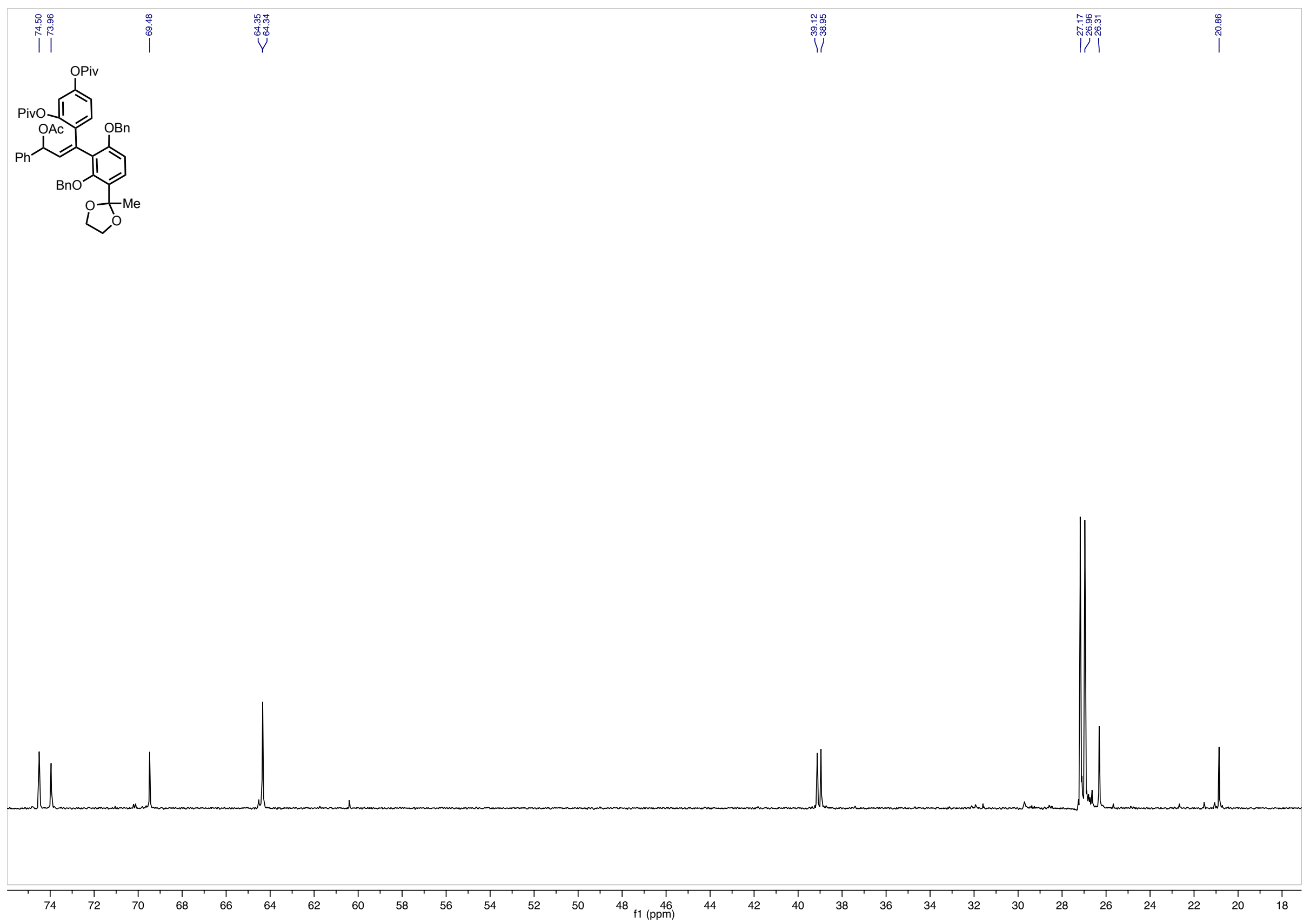

Figure S145. ${ }^{13} \mathrm{C}$ NMR (151 MHz, chloroform- $d$ ), SI-5 (inset). 

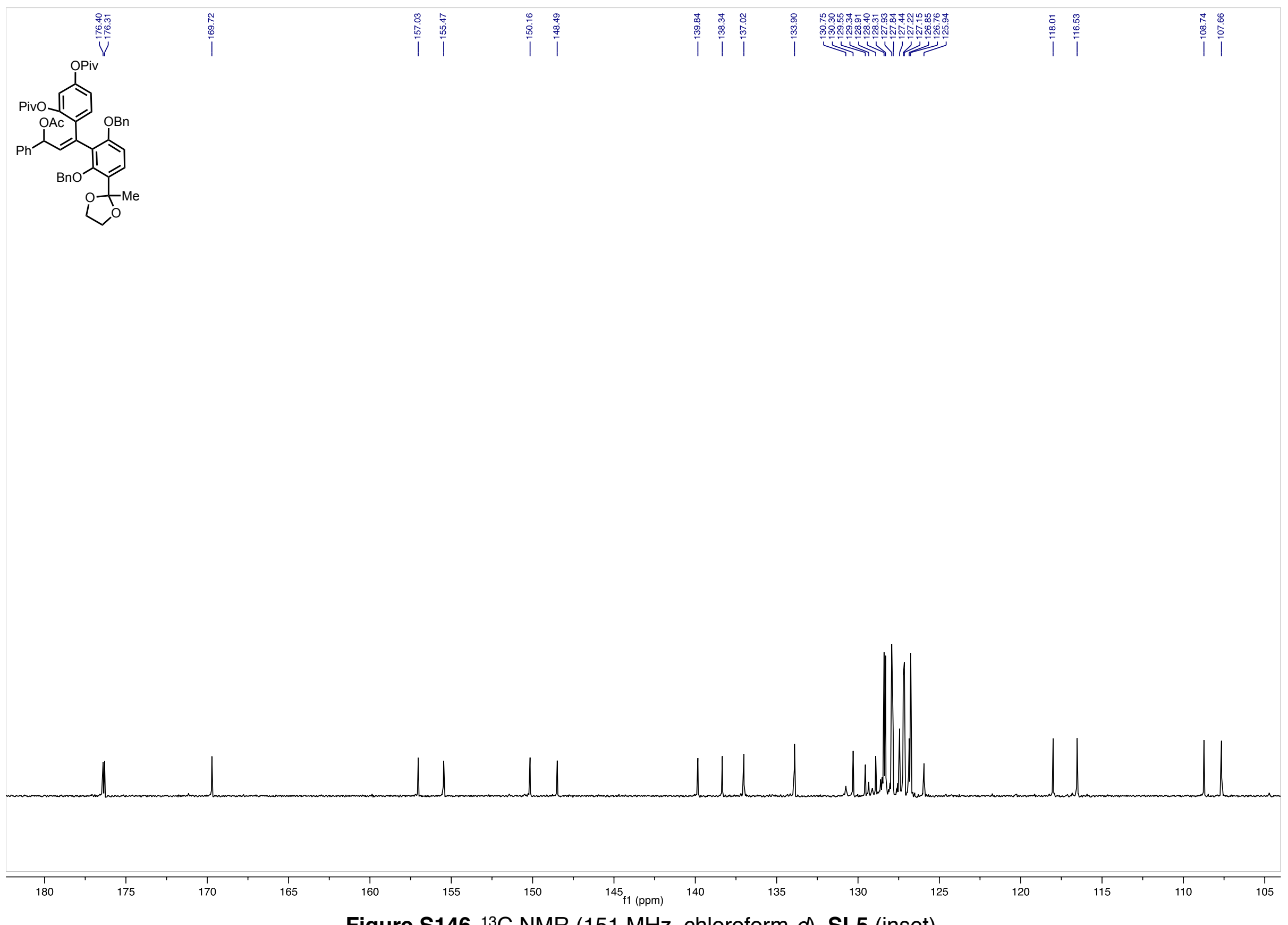

Figure S146. ${ }^{13} \mathrm{C}$ NMR (151 MHz, chloroform-d), SI-5 (inset). 


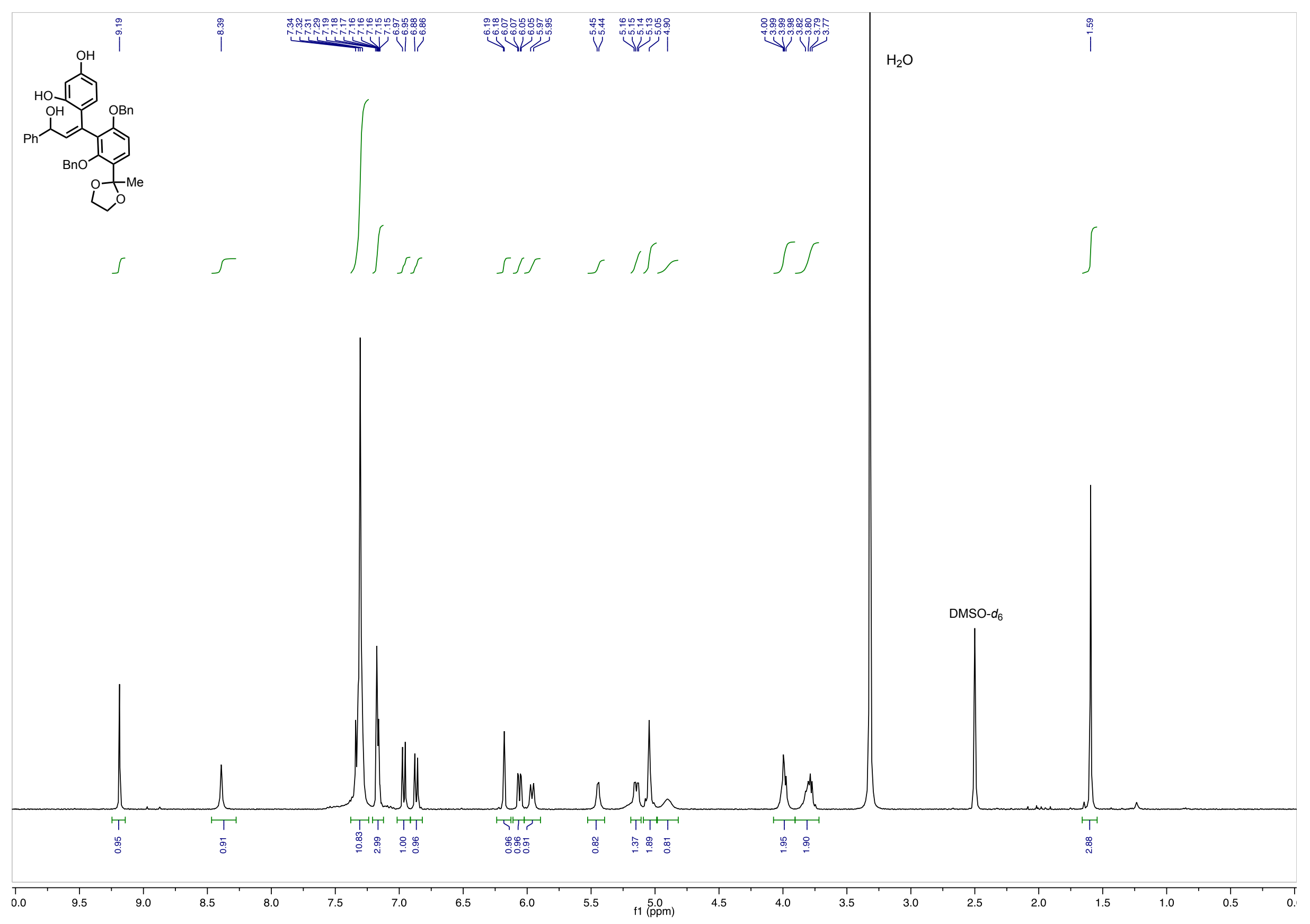

Figure S147. ${ }^{1 H} \mathrm{NMR}$ (600 MHz, DMSO- $d_{6}$ ), SI-6. 


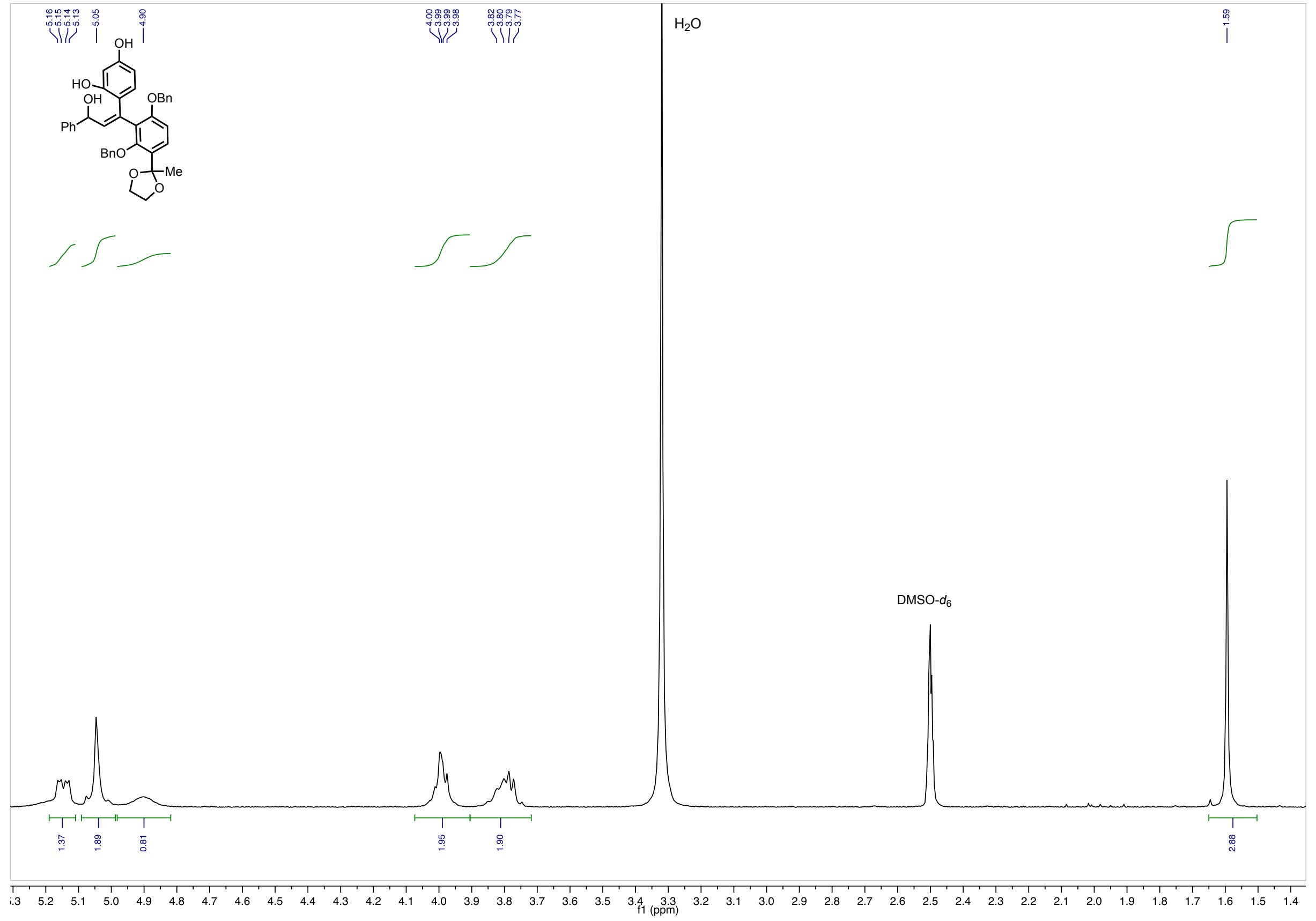

Figure S148. ${ }^{1} \mathrm{H}$ NMR (600 MHz, DMSO- $\left.d_{6}\right)$, SI-6 (inset).

S204 


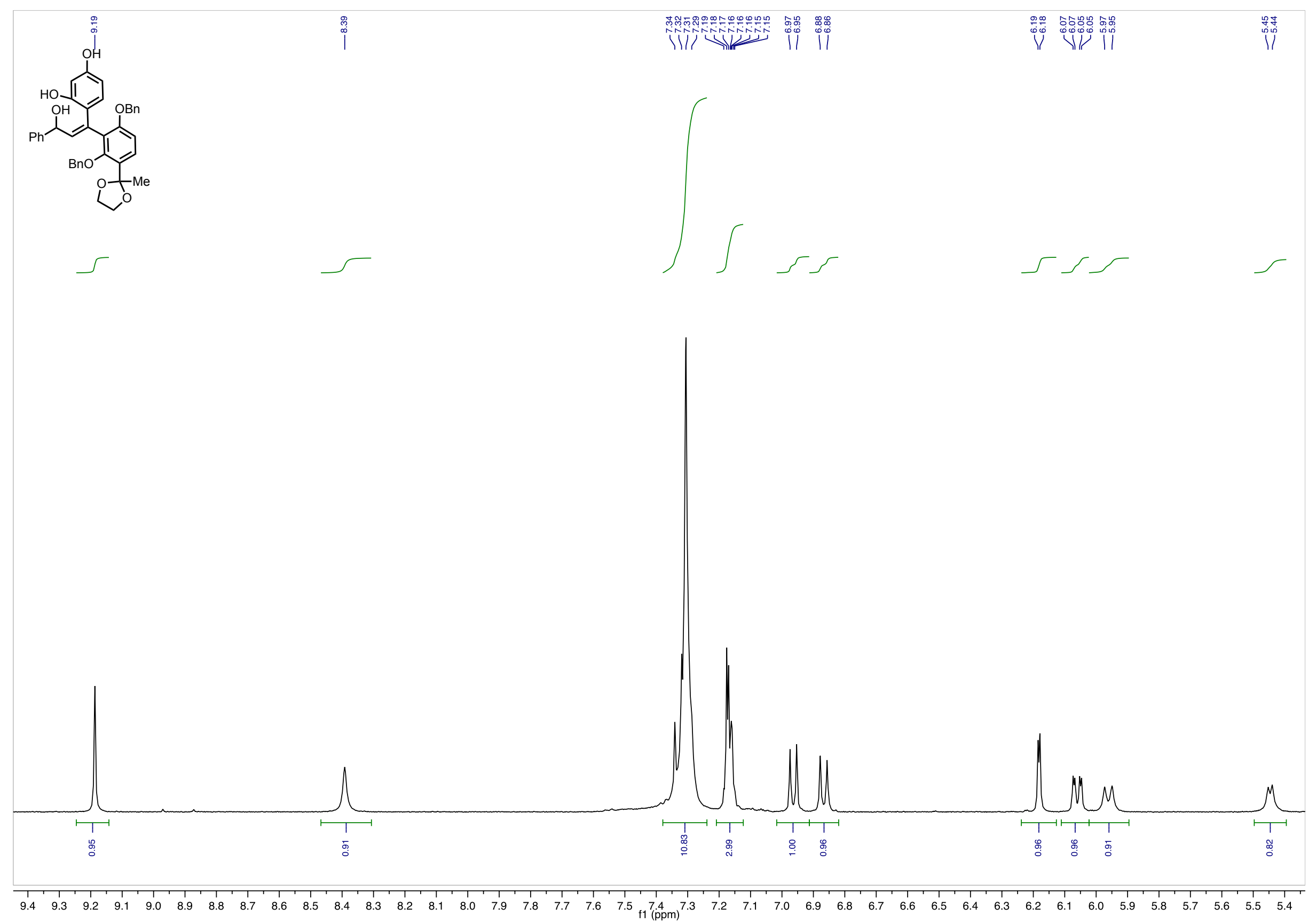

Figure S149. ${ }^{1} \mathrm{H}$ NMR (600 MHz, DMSO-d6), SI-6 (inset). 


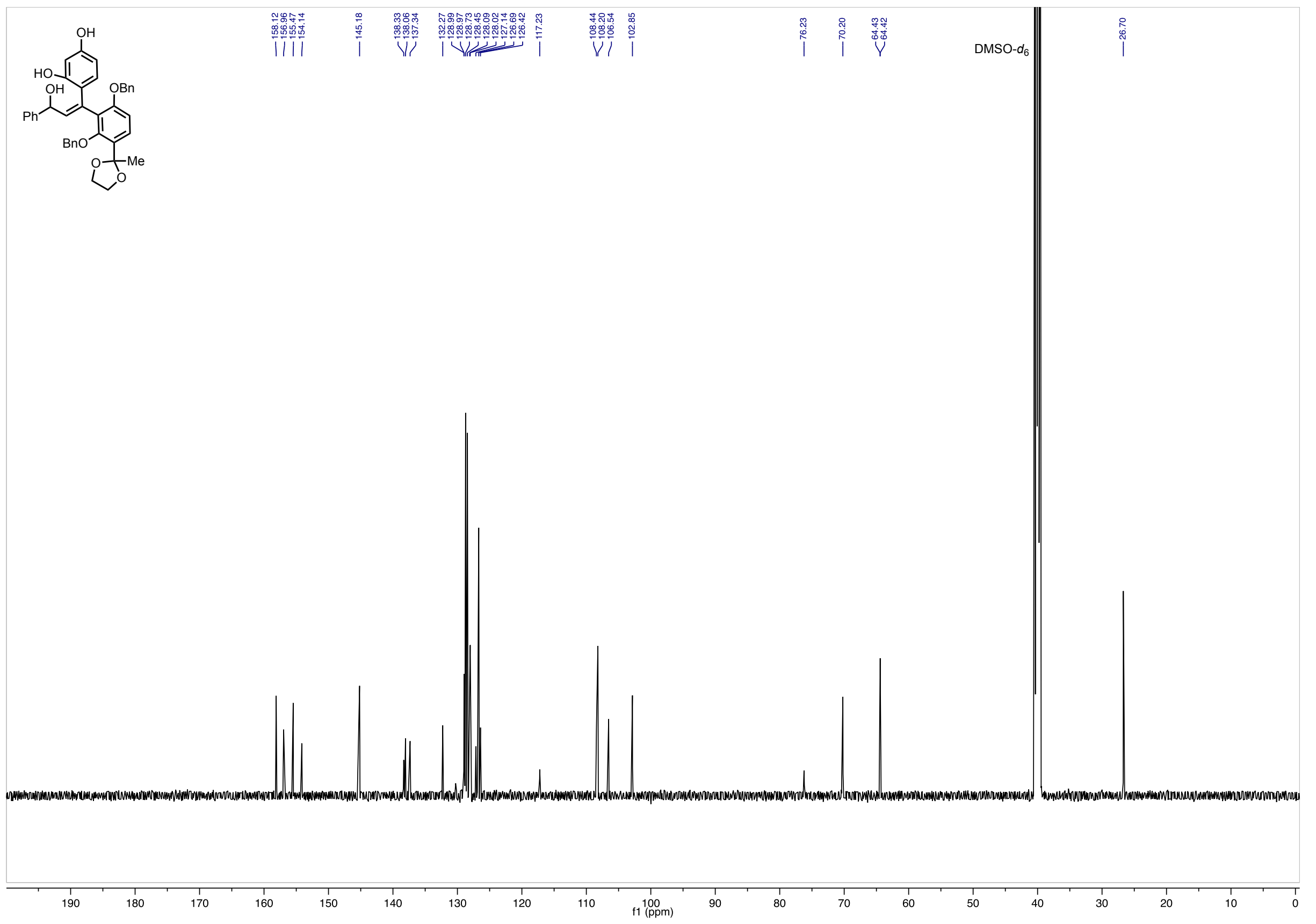

Figure S150. ${ }^{13} \mathrm{C}$ NMR (151 MHz, DMSO-d $d_{6}$, SI-6.

S206 


$$
1
$$



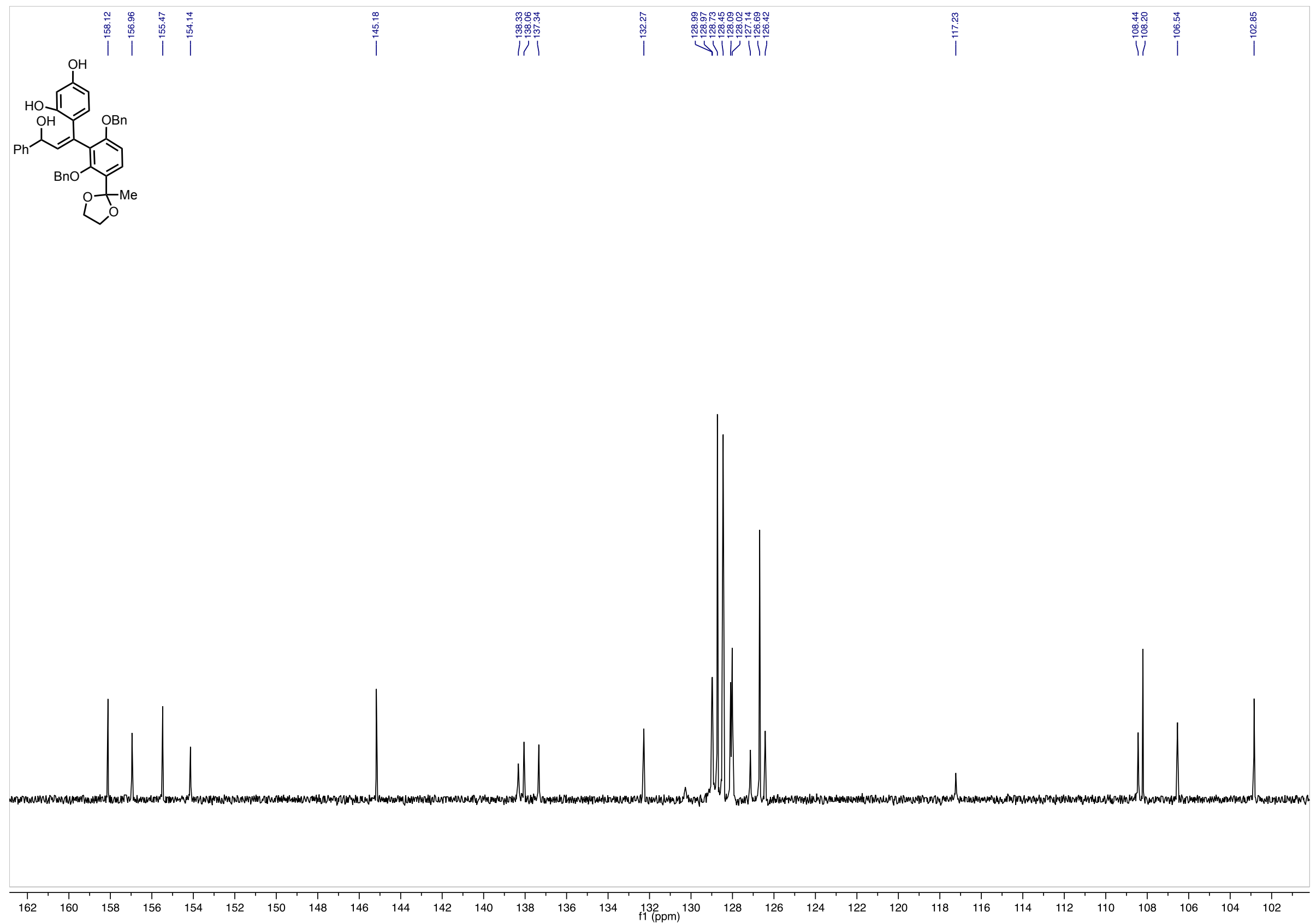

Figure S152. ${ }^{13} \mathrm{C}$ NMR (151 MHz, DMSO- $\left.d_{6}\right)$, SI-6 (inset). 


\section{References:}

S1) Bruno, N. C.; Tudge, M. T.; Buchwald, S. L.; Design and preparation of new palladium precatalysts for $\mathrm{C}-\mathrm{C}$ and $\mathrm{C}-\mathrm{N}$ cross-coupling reactions. Chem. Sci. 2013, 4, 916-920.

S2) Holder, J. C.; Marziale, A. N.; Gatti, M.; Mao, B.; Stoltz, B. M. Palladium-Catalyzed Asymmetric Conjugate Addition of Arylboronic Acids to Hetereocyclic Acceptors. Chem. Eur. J. 2013, 19, 74.

S3) Khilevich, A.; Bin, L.; Mayhugh, D. R.; Schkeryantz, J. M.; Zhang, D. US2010/0016373 A1, 2010.

S4) Serdiuk, I. E.; Roshal, A. D. Single and double intramolecular proton transfers in the electronically excited state of flavone derivatives. RSC Adv., 2015, 5, 102191-102203.

S5) Maloney, D. J.; Deng, J-.; Z.; Stark, S. R.; Gao, Z.; Hecht, S. M. (+)-Myristinin A, a Naturally Occurring DNA Polymerase Inhibitor and Potent DNA-Damaging Agent. J. Am. Chem. Soc. 2005, 127, 4140. b) Li, K.; Vanka, K.; Thompson, W. H.; Tunge, J. A. Stereochemical Control in the Reduction of 2-Chromanols. Org. Lett. 2006, 8, 4711

S6). Zhang, L-. J.; Bi, D-. W.; Hu, J.; Mu, W-. H.; Li, Y-. P.; Xia, G-. H.; Yang, L.; Liang, X-. S.; Wang, L.Q. Four Hybrid Flavan-Chalcones, Caesalpinnone A Possessing a 10,11-Dioxatricyclic [5.3.3.01,6]Tridecane-Bridged System and Caesalpinflavans A-C from Caesalpinia enneaphylla Org. Lett. 2017, 19, 4315.

S7) a) Van de Water, R. W.; Hoarau, C.; Pettus, T. R. R. Oxidative dearomatization of resorcinol derivatives: useful conditions leading to valuable cyclohexa-2,5-dienones. Tetrahedon Lett. 2003, 44, 5109-5113. b) Wenderski, T. A.; Hoarau, C.; Mejorado, L.; Pettus, T. R. R. Dearomatization applications of $\mathrm{I}(\mathrm{III})$ reagents and some unusual reactivity amongst resorcinol derived cyclohexadienones. Tetrahedron 2010, 66, 5873-5883. c) Mejorado, L. H.; Hoarau, C.; Pettus, T. R. R. Diastereoselective Dearomatization of Resorcinols Directed by a Lactic Acid Tether: Unprecedented Enantioselective Access to $p$-Quinols. Org. Lett. 2004, 6, 1535-1538. 